\title{
Alternative Electrochemical Salt Waste Forms, Summary of FY11-FY12 Results
}

Fuel Cycle Research \& Development

Prepared for

U.S. Department of Energy B.J. Riley, J.S. McCloy, J.V. Crum, W.C. Lepry, C.P. Rodriguez, C.F. Windisch Jr., J. Matyas, M.P. Westman, B.T. Rieck, J.B. Lang, M.J. Olszta, and D.A. Pierce Pacific Northwest National Laboratory January 17, 2014 FCRD-SWF-2013-000025, Rev.1 PNNL-22034 


\section{DISCLAIMER}

This information was prepared as an account of work sponsored by an agency of the U.S. Government. Neither the U.S. Government nor any agency thereof, nor any of their employees, makes any warranty, expressed or implied, or assumes any legal liability or responsibility for the accuracy, completeness, or usefulness, of any information, apparatus, product, or process disclosed, or represents that its use would not infringe privately owned rights. References herein to any specific commercial product, process, or service by trade name, trade mark, manufacturer, or otherwise, does not necessarily constitute or imply its endorsement, recommendation, or favoring by the U.S. Government or any agency thereof. The views and opinions of authors expressed herein do not necessarily state or reflect those of the U.S. Government or any agency thereof. 


\section{SUMMARY}

The Fuel Cycle Research and Development Program, sponsored by the U.S. Department of Energy Office of Nuclear Energy, is currently investigating alternative waste forms for wastes generated from nuclear fuel processing. One such waste results from an electrochemical separations process, called the "Echem" process. The Echem process utilizes a molten $\mathrm{KCl}-\mathrm{LiCl}$ salt to dissolve the fuel. This process results in a spent salt containing alkali, alkaline earth, lanthanide halides and small quantities of actinide halides, where the primary halide is chloride with a minor iodide fraction. Pacific Northwest National Laboratory (PNNL) is concurrently investigating two candidate waste forms for the Echem spent-salt: high-halide minerals (i.e., sodalite and cancrinite) and tellurite $\left(\mathrm{TeO}_{2}\right)$-based glasses. Both of these candidates showed promise in fiscal year (FY) 2009 and FY2010 with a simplified nonradioactive simulant of the Echem waste. Further testing was performed on these waste forms in FY2011 and FY2012 to assess the possibility of their use in a sustainable fuel cycle. This report summarizes the combined results from FY2011 and FY2012 efforts.

The chemical durability results for the tellurite glasses in FY2010 revealed a wide variation of waste form performance in these materials. The most promising glass studied was a lead-tellurite glass formulation with the Echem salt simulant. This glass showed very good chemical durability with a normalized release for sodium of $0.478 \mathrm{~g} / \mathrm{m}^{2}$ following a seven-day product consistency test. With this in mind, the following studies were undertaken in FY2011 and FY2012 to further investigate and optimize this type of glass as a potential waste form:

1) Expanded study on lead-tellurite glasses. The lead-tellurite glass system was further investigated to determine the waste-loading limits and assess the tellurium-oxygen bonding structure as a function of waste loading by characterization with nuclear magnetic resonance, Raman spectroscopy, and X-ray diffraction. The Te-O network in tellurite glass is quite complex where at least eight different structures have been presented in the literature and typically include $\mathrm{TeO}_{4}$, $\mathrm{TeO}_{3+1}$, and $\mathrm{TeO}_{3}$ structures present in either a trigonal bipyramidal or trigonal pyramidal arrangement. The structural investigation revealed that the medium-range order in the glass decreased and the tellurite network was depolymerized as the waste fraction was increased. It was apparent that glass-network breaking was predominantly caused by the additions of the waste cations and that the presence of chloride may have actually caused some repolymerization of the network by scavenging alkali and preventing it from acting as a network modifier. At the highest waste loadings, the glass became phase-separated, resulting in a Te-rich phase and a Pb-alkalichloride-rich phase, and some chloride-containing crystals were formed. We also investigated some of the other waste-form related properties of the lead-tellurite glasses, such as the thermal diffusivity, glass transition temperature, crystallization temperature, melting temperature, and mass loss as a function of temperature.

2) Investigated the glass network structure of P-series glasses from FY2010 with Raman spectroscopy. Here, we studied the glass structure of the $\mathrm{P}$-series glasses $\left(\mathrm{TeO}_{2}\right.$ with various other oxide additions such as $\mathrm{ZnO}, \mathrm{PbO}, \mathrm{P}_{2} \mathrm{O}_{5}, \mathrm{Al}_{2} \mathrm{O}_{3}, \mathrm{~B}_{2} \mathrm{O}_{3}$, and $\mathrm{WO}_{3}$ ); the study provided a preliminary understanding of the various Te-O structural configurations in the literature and how to identify them with Raman spectroscopy. The goal was to correlate a particular Te-O structure with a glass that had high chemical durability and reverse-engineer a glass with very high chemical durability by targeting the preferred structure with changes in composition. This has proved unsuccessful overall, but we did observe some unique frequency bands in the Raman spectra for the lead-tellurite glasses that might be a first step toward better understanding the link between the glass structure and the chemical durability.

3) Investigated the structural effects of various components in the tellurite glasses. For this study, we used Raman spectroscopy to look at the structure of simplified glasses in which various components had been removed and added to provide different combinations of the anions and 
cations. This study provided some very useful information regarding the effects of $\mathrm{Cl}$ and alkalis on the tellurite glass network.

4) Investigated polycrystalline tellurite compounds as a structural surrogate for tellurite glass. We found a previous study where a large set of tellurite crystals were fabricated and the Te-O structures determined with nuclear magnetic resonance (NMR). Single-phase tellurite crystals have a single type of tellurium-oxygen structure, in contrast with tellurite glasses that have a mixture of different types of Te-O bonding configurations. The goal was to make a series of tellurite crystals from each category and assess the chemical durability of each. In FY2011, we made a series of crystals, some of which were highly phase-pure ( $>95 \%)$. We intended to eventually verify their structure (with NMR and/or Raman spectroscopy) and perform chemical durability tests on them to look for a correlation between the structure and the chemical durability but fabrication was more difficult than expected so budget did not allow for further testing at this point.

For the high-halide minerals, we looked at optimizing a few of the variables in the solution-based process as well as some post-process adjustments to the heat-treatments. These variable studies included the following:

1) Alternative silica sources. We fabricated sodalites with an organic source of silica, tetraethyl orthosilicate (TEOS), in place of the colloidal silica. The goal with this substitution was to assess the differences in reaction rates for sodalite formation among the silica sources as well as the differences in sodalite concentration. The change in silica sources resulted in a noticeable improvement from 23.8 mass\% sodalite in the S5A specimen with colloidal silica to 95.7 mass\% sodalite in the S5C specimen with TEOS.

2) Alternative 4-coordinated network formers. We performed the solution-based process with germanium (IV) ethoxide, an organic form of $\mathrm{Ge}^{4+}$, in place of the TEOS to see whether we could fabricate a germanium-based sodalite. The germanium sodalite was targeted because it was predicted to (1) reduce the firing temperature resulting in an increased sodalite concentration because of the reduced chloride volatility (due to lower firing temperature) and (2) increase firedpellet density improving volumetric waste loading. The high porosity in the initially fired pellets was a deterrent to pursuing this route any further, though we did demonstrate a high germaniabased sodalite concentration ( 82 mass $\%$ ) in the as-made gel. The high porosity in conjunction with the high cost of germanium (IV) ethoxide led us to abandon this pursuit.

3) Alternative sintering aids. We fabricated a series of six alternative sintering aids with different viscosities in order to consolidate the solution-derived powders at a lower temperature, retaining the sodalite structure and preventing chloride volatility. These sintering aids included four sodium borosilicate glasses, a sodium aluminophosphate glass, and a lead-tellurite glass. Each of these glasses was added to a separate batch, as a powder; the gel was dried, specimens from the batches were pressed into pellets, fired at different temperatures, and the phases quantified with $\mathrm{X}$-ray diffraction. The sodium borosilicate glasses dramatically improved the densification ( $\leq 96 \%$ of theoretical density) of the fired pellets at much-reduced temperatures of $\sim 750{ }^{\circ} \mathrm{C}$, where rather large amounts of sodalite were still produced (70.6 mass $\%)$.

4) Alternative approaches to making sodalite. In FY2012, we took a different approach to making high density sodalite pellets. Three different solution-based approaches were evaluated to make sodalite with the same simulated $X \mathrm{Cl}_{20}$ salt. Several aspects were varied during the process including: the reactants, the amount of sintering aid added $(0,5$, and 10 mass $\%)$, the step in the process when the sintering aid was added, calcination, and the timing of the calcination step in the process. The three sodalite methods ( $S M$-\#) used in the current study are $S M-1, S M-2$, and $S M-3$, simplified below. The equations above are listed with the salt being denoted as "NaCl" but, in fact, the actual salt compositions contain $\mathrm{R}^{1+}, \mathrm{R}^{2+}$, and $\mathrm{R}^{3+}$ cations so the $\mathrm{R}: \mathrm{Cl}$ ratio is not $1: 1$ $(\mathrm{R}: \mathrm{Cl}<1$, by mole). The reactants were added in the proper amounts to obtain the targeted value 
for $\mathrm{Al}: \mathrm{Cl}(3: 1)$ and $\mathrm{Si}: \mathrm{Cl}(3: 1)$, on a molar basis, where the alkali fraction $\left(\mathrm{R}^{1+}\right)$ was intentionally below the target of $4: 1\left(\mathrm{R}^{1+}: \mathrm{Cl}\right)$ to help influence the incorporation of alkaline earth $\left(\mathrm{R}^{2+}\right)$ and $\mathrm{Ln}$ $\left(\mathrm{R}^{3+}\right)$ components from the $X \mathrm{Cl}_{20}$ into the sodalite structure. The results from this study showed that each formulation had advantages and disadvantages where SM-3 pellets had high density and low sodalite production and SM-2 pellets had very high sodalite production with modestly high densities. A mixture of these methods will likely be considered in FY2013.

SM-1: $\quad 6 \mathrm{NaOH}+6 \mathrm{Al}(\mathrm{OH})_{3}+2 \mathrm{NaCl}+6 \mathrm{SiO}_{2} \rightarrow \mathrm{Na}_{8}\left(\mathrm{AlSiO}_{4}\right)_{6} \mathrm{Cl}_{2}+24 \mathrm{OH}^{-}$

SM-2: $\quad 6 \mathrm{NaAlO}_{2}+2 \mathrm{NaCl}+6 \mathrm{SiO}_{2} \rightarrow \mathrm{Na}_{8}\left(\mathrm{AlSiO}_{4}\right)_{6} \mathrm{Cl}_{2}$

SM-3: $\quad 6 \mathrm{NaOH}+3 \mathrm{Al}_{2} \mathrm{Si}_{2} \mathrm{O}_{7}+2 \mathrm{NaCl} \rightarrow \mathrm{Na}_{8}\left(\mathrm{AlSiO}_{4}\right)_{6} \mathrm{Cl}_{2}+6 \mathrm{OH}^{-}$ 


\section{CONTENTS}

SUMMARY iii

1 INTRODUCTION 1

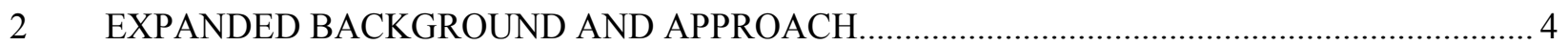

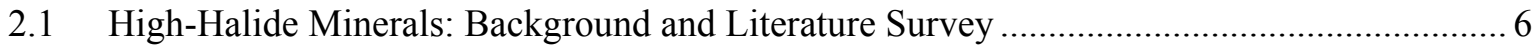

2.2 Tellurite Glasses: Background .................................................................................. 9

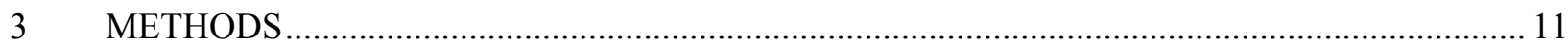

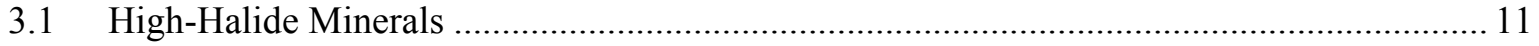

3.1.1 Solution-Based Fabrication Method for Halide Minerals in FY2010-FY2011 ........ 11

3.1.2 Solution-Based Fabrication Method for Halide Minerals in FY2012 ….................... 13

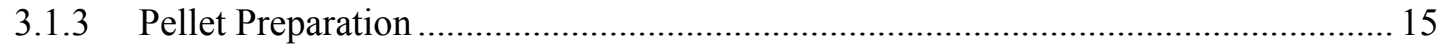

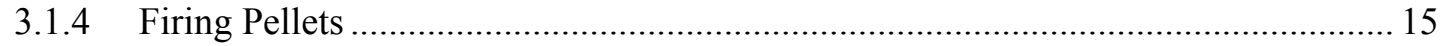

3.1.5 Cold-Pressing Pressure Variation Study (PVS) and Evaluation of Method

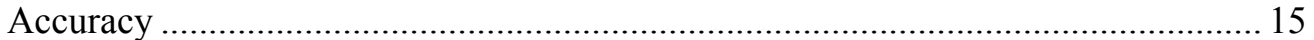

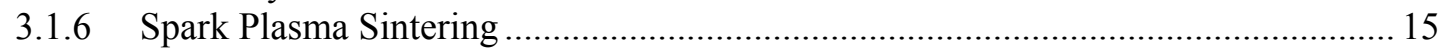

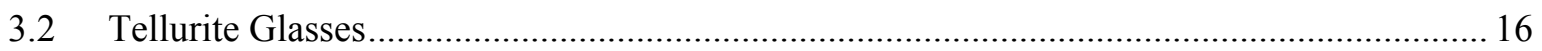

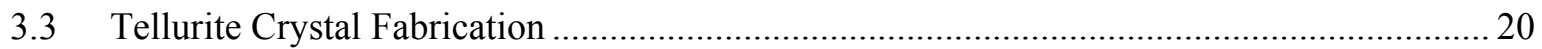

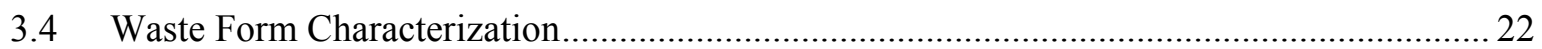

3.4.1 Optical Microscopy and Photo-Documentation......................................................... 22

3.4.2 Helium Pycnometry - Density Measurements for Tellurite Glasses ........................ 22

3.4.3 Density and Porosity Measurements for High-Halide Minerals ............................... 23

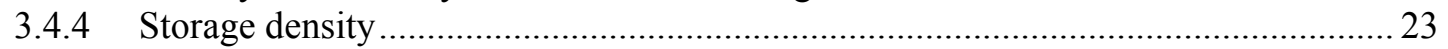

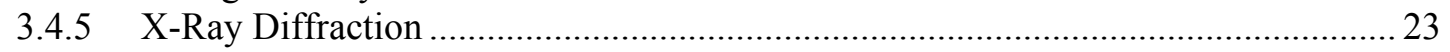

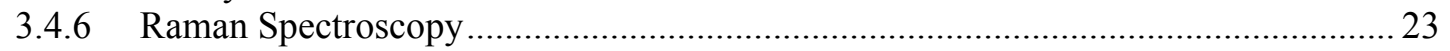

3.4.7 Chemical Durability with the Product Consistency Test (PCT) .............................. 24

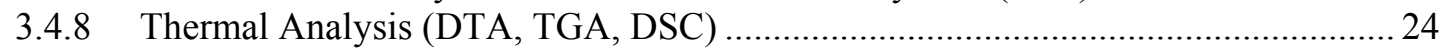

3.4.9 Scanning Electron Microscopy and Energy Dispersive Spectroscopy (SEM-

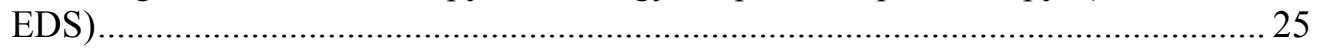

3.4.10 Transmission Electron Microscopy and Energy Dispersive Spectroscopy

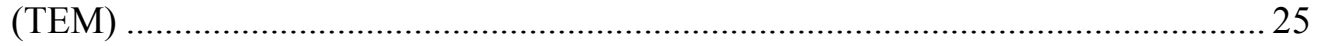

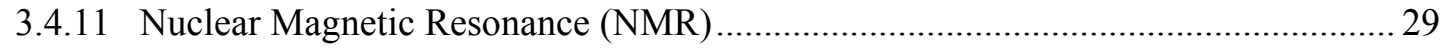

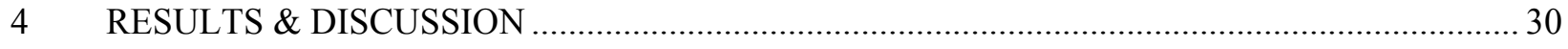

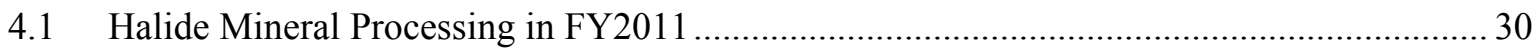

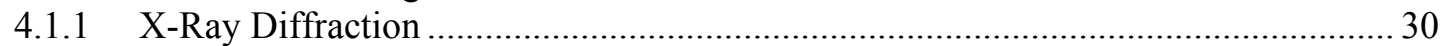

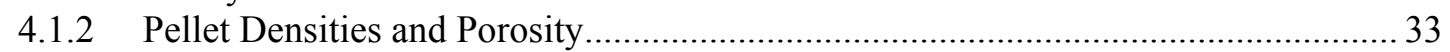

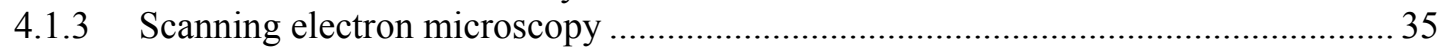

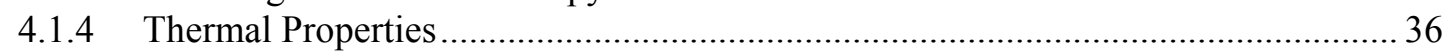

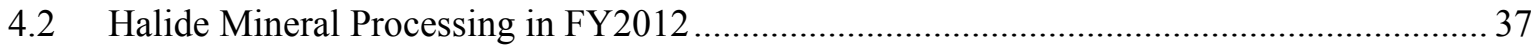

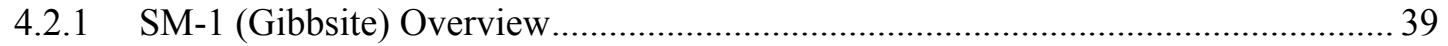

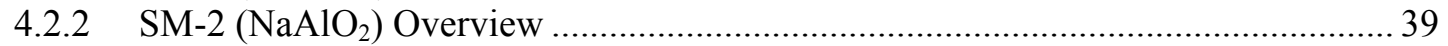

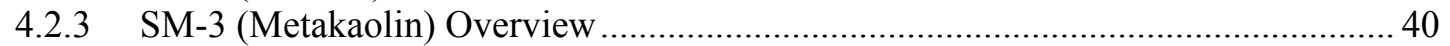

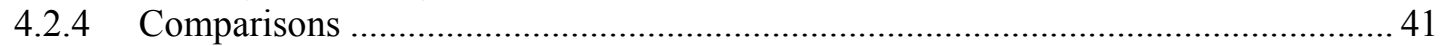

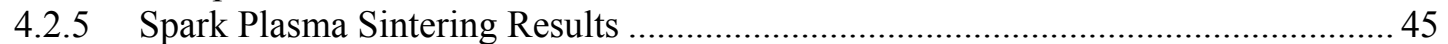

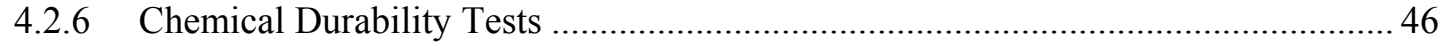




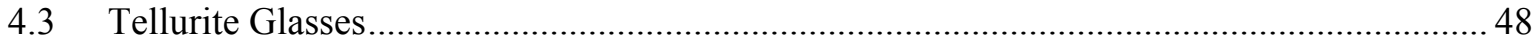

4.3.1 Tellurite Glasses: Compositional Variation Study ................................................ 48

4.3.2 Tellurite Glasses: Lead-Tellurite and Structural Variation Glasses Study................. 58

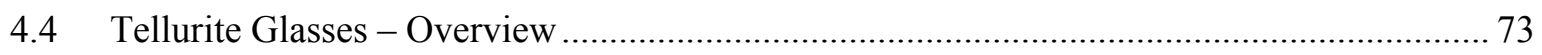

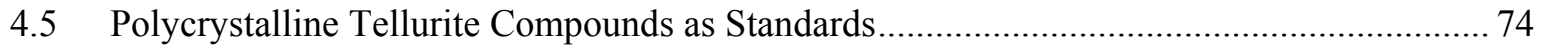

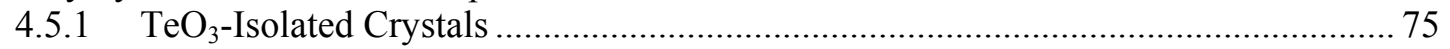

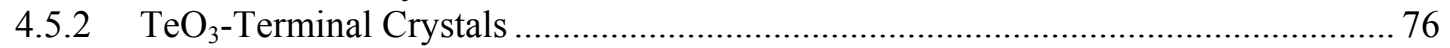

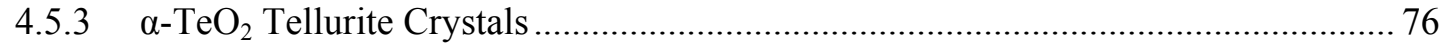

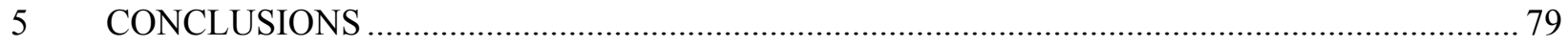

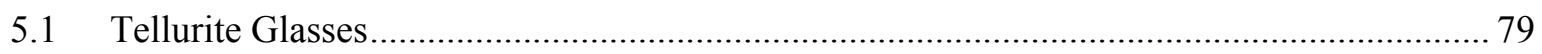

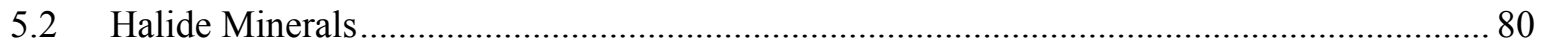

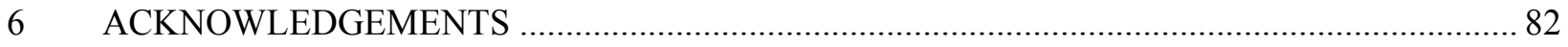

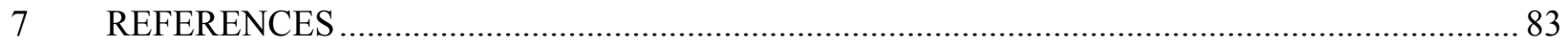

Appendix A: Structural Notations in Tellurite Glass ..................................................................... A.1

\section{FIGURES}

Figure 1.1. Ranges of isotropic chemical shifts $\left(\delta_{\text {iso }}\right)$ for tellurium sites with different structural units determined with ${ }^{125} \mathrm{Te}$ magic angle spinning (MAS) NMR at $9.4 \mathrm{~T}$ - recreated from Sakida et al. (1999a).

Figure 1.2. (a) Hydrocarbon stick figure of TEOS, (b) hydrocarbon stick figure of germanium(IV) ethoxide, and (c) a three-dimensional view of these structures. Note: for (a) and (b), the atom located at each bend and terminal is a $C$ atom where each $C$ has four total bonds achieved with hydrogen atoms in areas where oxygen is not present.

Figure 3.1. Schematic of the simplified solution-based process.

Figure 3.2. Simulated amorphous patterns of a few tellurite crystals. These patterns were generated in JADE by setting the crystallite size to $10 \AA$ and putting $0 \%$ strain on the network. These were used to predict the amorphous structure of the crystalline compounds.

Figure 3.3. Cast cylinders of $\mathrm{P}-11$ glass from which discs were cut for the thermal diffusivity measurements.

Figure 4.1. Summary of phase distribution in S5A specimens at different firing temperatures (mass \%).....

Figure 4.2. Summary of phase distribution in the S5A, S5A after drying for 5-days at $90{ }^{\circ} \mathrm{C}$, $\mathrm{S} 5 \mathrm{C}$, and S5D formulations as-made (unfired) (mass \%). 30

Figure 4.3. Phase distribution for the various S5C formulations at fired at different temperatures. The legend presented in the upper left figure is valid for all figure parts. .31

Figure 4.4. Summary of phase analysis for the as-made S5D material. 32

Figure 4.5. (a) Summary of $\%$ of $\rho_{\mathrm{t}}$ as a function of firing temperature for S5C series specimens made with an additional 5 mass $\%$ of various sintering aids (see Table 3.2 for more 
details). (b) Column chart comparing the mass $\%$ of sodalite in each specimen with the $\%$ of $\rho_{\mathrm{t}}$ for all S5C specimens fired at $750{ }^{\circ} \mathrm{C}$. 33

Figure 4.6. Comparison of $\rho_{\mathrm{t}}$ and mass\% sodalite for the S5A, S5B, S5C, S6, and C1/C2 series specimens (at mixed firing temperatures). The dotted blue line outlines the maxima boundary for TEOS-based pellets.

Figure 4.7. SEM micrograph and EDS dot map of S6 pellet with 7\% NBS-1 glass (S6$\left.7 \% 750^{\circ} \mathrm{C}\right)$

Figure 4.8. SEM micrographs of S4A, S5C, and a dot map on S5C (TEOS-based) showing elemental distribution and a clear distinction between the chloride-containing phase (sodalite), the phase without chloride (nepheline), and the lanthanide phase (shown as La).

Figure 4.9. Thermal diffusivity of Sodalite 5A pellets. Error bars for the measured thermal diffusivity are included on the data points

Figure 4.10. Summary of density (\% of theoretical) as a function of sodalite concentration (mass\%) for all data. The filled squares are the PVS specimens with 5\% SA800 that were isostatically pressed at $3.4 \times 10^{8} \mathrm{~Pa}\left(5.0 \times 10^{4} \mathrm{PSI}\right)$.

Figure 4.11. XRD summary figure showing sodalite (space group $=P 43 n$ ) as the primary phase for these three specimens SM1-5\%-C-850 ${ }^{\circ} \mathrm{C}$, SM2-5\%-C-850 ${ }^{\circ} \mathrm{C}$, and SM3-5\%-C$750^{\circ} \mathrm{C}$ with a unit cell of $a=0.882 \mathrm{~nm}, 0.889 \mathrm{~nm}$, and $0.887 \mathrm{~nm}$, respectively (Barth, 1932a). The minor phases were $\mathrm{KAlO}_{2}$ (Sokolowski and Kotarba, 2000) and $\mathrm{K}_{1.6} \mathrm{Na}_{6} \mathrm{Al}_{7.49} \mathrm{Si}_{8.51} \mathrm{O}_{32}$ (nepheline) (Tait et al., 2003a) for SM3-5\%-C-750 ${ }^{\circ} \mathrm{C}$. This plot shows the raw measured data (blue), fitted/calculated spectra (red), and difference patterns (green) for each spectra.

Figure 4.12. SEM-EDS dot map on SM2-5-C-850 ${ }^{\circ} \mathrm{C}$ showing compositional variations in the sodalite.

Figure 4.13. (left) Cluster phase analysis of the dot map seen in Figure 4.12 and (right) a comparison of the measured normalized compositions for the red and blue phase compared to the target values based on the target sodalite composition (oxygen is normalized out).

Figure 4.14. SEM micrographs showing a particle size comparison between the as-made powders for SM-1, SM-2, and SM-3 formulations.

Figure 4.15. Comparison plots for density ( $\%$ of theoretical density) and the mass $\%$ sodalite of specimens sorted by method and heat-treatment temperature.

Figure 4.16. Comparison plots between like procedures for the density (\% of theoretical maximum for sodalite) and the mass $\%$ sodalite of pellets fired at all temperatures for specimens with 5 and 10 mass\% additions of sintering aid (SA800).

Figure 4.17. Comparison of mass loss during firing for calcined versus uncalcined specimens. Here, mass loss is determined by $100 \cdot\left(m_{\mathrm{i}}-m_{\mathrm{f}}\right) / m_{\mathrm{i}}$ (to get \%) where $m_{\mathrm{i}}$ is the initial mass and $m_{\mathrm{f}}$ is the final mass as measured on an analytical balance. Dotted lines are a guide for unity.

Figure 4.18. Comparison plots between like procedures for the density (\% of theoretical maximum for sodalite) and the mass $\%$ sodalite of as-made pellets without sintering aids fired at all temperatures (i.e., SM\#-AM and SM\#-AM-C). The $45^{\circ}$ lines are a guide for unity. 
Figure 4.19. Comparison plots for the density (\% of theoretical density) and the mass $\%$ sodalite of specimens that were and were not calcined prior to being pressed into pellets (for all temperatures). Legends in the top row of figures are the same for the bottom row. 44

Figure 4.20. Comparisons from the pressure variation study showing the density and sodalite as a function of pressing pressure.

Figure 4.21. Density versus sodalite for the PVS samples. SM-1, SM-2, and SM-3 specimens are in red, green, and blue, respectively.

Figure 4.22. Spark plasma sintering results. These show the displacement and temperature as a function of time for each pellet. 46

Figure 4.23. (a) XRD summary plot showing the amorphous background fits for all glasses before PCT for comparison with three amorphous simulated patterns for $\mathrm{TeO}_{2}$ PDF structures (74-1131, 42-1365, and 78-1713; see Table 4.10). Vertical lines are added as a visual guide for the location of the primary amorphous humps. The spectra are vertically offset (intensities retained) to accentuate the differences so the vertical scale is listed in arbitrary units (a.u.). (b) XRD spectra for all 10 glasses after PCT. Best-fit diffraction peaks were identified and are denoted by symbols near the most intense of the diffraction peaks for each phase observed for each glass. Here, - denotes tetragonal $\alpha-\mathrm{TeO}_{2}, \mathrm{O}$ denotes orthorhombic $\beta-\mathrm{TeO}_{2}, \boldsymbol{\square}$ denotes $\mathrm{Pb}_{2} \mathrm{Te}_{3} \mathrm{O}_{7}, \square$ denotes $\mathrm{Na}_{2} \mathrm{Te}_{2} \mathrm{O}_{7}$, denotes $\mathrm{Ba}_{3} \mathrm{WO}_{6}$, and $\diamond$ denotes $\mathrm{Zn}_{2} \mathrm{Te}_{3} \mathrm{O}_{8}$. This figure was recreated from our previous work (Riley et al., 2010).

Figure 4.24. Examples of curve fitting for $(\mathrm{A}) \mathrm{Pb}(\mathrm{P}-8)$ and $(\mathrm{B}) \mathrm{Pb}+\mathrm{Cl}(\mathrm{P}-11)$ glasses. .52

Figure 4.25. Raw Raman spectra without backgrounds removed for glasses with and without $\mathrm{XCl}_{20}$ (listed as "Cl"). Spectra are not offset but are shown as collected unless otherwise noted. (a) PbO-glasses P-8 (bottom, black) and P-11 (top, red, intensity 5× from measured); (b) $\mathrm{Al}_{2} \mathrm{O}_{3} / \mathrm{B}_{2} \mathrm{O}_{3}$-glasses P-7 (top, black) and P-2 (bottom, red, offset $+100,000$ counts); (c) $\mathrm{WO}_{3}$-glasses P-9 (top, black) and P-12 (bottom, red, intensity $3 \times$ from measured); (d) $\mathrm{P}_{2} \mathrm{O}_{5}$-glasses P-5 (top, black) and P-14 (bottom, red); and (e) $\mathrm{ZnO}$ glasses P-4 (top, black) and P-13 (bottom, red).....

Figure 4.26. DTA/TGA results for (A) P-8 and (B) P-11 collected during heating...... .57

Figure 4.27. (A) Thermal diffusivity, $\alpha$, for tellurite glass, P-11, as well as PNNL Sodalite, 5A, as a function of temperature. (B) A P-8 specimen prepared for LFA measurements; it is slightly crystallized (see small, clear $\mathrm{Pb}_{2} \mathrm{Te}_{3} \mathrm{O}_{7}$ crystals throughout the bulk).

Figure 4.28. (a) Pictures of lead-tellurite glasses at different waste loadings. (b,c) A polished cross-section of the two phases present in the phase-separated T2G-1-PbO specimen (Riley et al., 2010). Note that $\mathrm{TPb}_{30}$ is not shown but looks identical to $\mathrm{TPb}_{2}$

Figure 4.29. Summary figure providing visual comparisons between the structural variation glasses. Here, T2G-21 was used as a placeholder for P-8 since they have identical compositions and appearances.

Figure 4.30. (a) Bulk density $\left(\rho_{\mathrm{b}}\right)$ and (b) storage density $\left(\rho_{\mathrm{sd}}\right)$ of the lead-tellurite glasses as a function of $\mathrm{XCl}_{20}$-loading. In (b) a comparison is given to the baseline glass-bonded sodalite.

Figure 4.31. Micro-Raman spectroscopy of separated phases in T2G-1-PbO (same composition as $\left.\mathrm{TPb}_{15}\right)$.

Figure 4.32. Raman spectra of $\mathrm{K}_{2} \mathrm{O}$ and $\mathrm{KCl}$ glasses (top) and some of the lead-tellurite glasses (bottom). 
Figure 4.33. Quantitative Raman analysis for the lead-tellurite glasses. (a) $t b p / t p$ area ratio from Raman spectra as a function of waste loading. A lower $(\mathbf{C}+\mathbf{D}) /(\mathbf{A}+\mathbf{B})$ ratio represents a more depolymerized network. (b) Fitted peak width as a function of loading, indicating site disorder in the tp part of the glass structure with increasing waste loading. In both figures, lines indicate averages where more than one data point (circle) is shown.

Figure 4.34. Summary of XRD results for lead-tellurite variation study glasses. The details of these phases can be found in Table 4.17.

Figure 4.35. (a) Background fits on raw XRD data from the lead-tellurite glasses presented in Figure 4.34. (b) Amorphous hump maxima locations in 20-locations denoted by vertical lines in (a) - along with linear data fits for informational purposes only.....

Figure 4.36. Scanning electron microscopy of yellow-white region in T2G-1-PbO showing nano-droplet phase-separation.

Figure 4.37. Energy dispersive spectroscopy on phase-separated region of $\mathrm{T} 2 \mathrm{G}-1-\mathrm{PbO}$ where the average composition of the dark droplets in Figure 4.36 is compared to that of the surrounding lighter matrix. The data is presented in both (a) atomic $\%$ and (b) mass $\%$. It should be noted that that Li cannot be detected with this technique and is likely present in the droplets along with the other alkali cations. We expect that the compositional values for the droplets are slightly skewed by the bulk matrix just below the droplets due to electron beam penetration into and interaction with the subsurface matrix.

Figure 4.38. $\mathrm{SEM}$ micrographs of $\mathrm{TPb}_{22.5}$ (left) showing semi-continuous salt phase formation and $\mathrm{TPb}_{30}$ (right) showing high density droplets.

Figure 4.39. TEM micrographs illustrating the evolution of beam-induced phase separation in $\mathrm{TeO}_{2}-\mathrm{PbO}-\mathrm{XCl}_{20}$ glasses $(10 \%, 14 \%)$. The $10 \mathrm{mass} \% \mathrm{XCl}_{20}$ specimen is completely amorphous. The 14 mass $\% X_{20}$ has phase separated regions $\sim 10 \mathrm{~nm}$ in diameter. Using a larger condenser aperture to image the specimen, beam-induced phase separation occurs (higher density of phase-separated regions which are slightly larger, $\sim 18 \mathrm{~nm}$ ).

Figure 4.40. TEM micrographs showing phase separation and crystallization in $\mathrm{TeO}_{2}-\mathrm{PbO}-X \mathrm{Cl}$ glasses $(17,22.5 \%)$. At $17.5 \% \mathrm{XCl}_{20}$ loading, full phase separation is observed, with phase separation on the order of $30-60 \mathrm{~nm}$ in diameter. At $22.5 \% \mathrm{XCl}_{20}$ loading, the phase separation is observed to be on the order of $300-400 \mathrm{~nm}$ in diameter.

Figure 4.41. Summary of the TGA results for the lead-tellurite glasses. Figure (a) shows the thermogravimetric loss as a function of temperature; the inset shows a magnified view of the high-temperature end of the plot. (b) Cumulative mass retention at $735^{\circ} \mathrm{C}$ for all of the glasses.

Figure 4.42. DSC results for the lead-tellurite glasses. Figure (a) shows the heat-flow curves as a function of temperature where the calculated $T_{\mathrm{g}}$ and $T_{\mathrm{c}(2)}$ are marked with vertical lines. Figure (b), plotted from the data in Table 4.18, shows the trends for $T_{\mathrm{c}(2)}$ (top), $T_{\mathrm{g}}$ (middle), and $T_{\mathrm{c}(2)}-T_{\mathrm{g}}$ (bottom); $\mathrm{TPb}_{14}$ did not fit well on any of the plots so it is denoted by solid symbols on each trend.

Figure 4.43. (left) ${ }^{125} \mathrm{Te} \mathrm{NMR}$ for $10 \% \mathrm{XCl}_{20}$ glass showing experiment (Exp), simulation (Sim), and site deconvolution. (right) ${ }^{207} \mathrm{~Pb}$ NMR results for $15 \% \mathrm{XCl}_{20}$ glass.

Figure 4.44. Summary of ${ }^{125} \mathrm{Te},{ }^{35} \mathrm{Cl},{ }^{207} \mathrm{~Pb}$, and ${ }^{23} \mathrm{Na} \mathrm{NMR}$ as a function of waste loading. Spectra are aligned by glasses and the gaps show were data was not collected......................... 70

Figure 4.45. Variation of $\mathrm{Pb}$ site distribution with $\mathrm{XCl}_{20}$-loading.................................................... 72 
Figure 4.46. Pictures of the tellurite crystals fabricated in FY2011. The scale-bar is the same for all micrographs.

Figure 4.47. Rietveld refinement on $\mathrm{ZnTeO}_{3}$ crystals showing the measured, calculated, and difference spectra. Here, - represents the target phase $\left(\mathrm{ZnTeO}_{3}, \mathrm{PDF} \# 72-1410\right.$, Table 4.21).

Figure 4.48. Rietveld refinement on $\mathrm{Zn}_{2} \mathrm{Te}_{3} \mathrm{O}_{8}$ crystals showing the measured, calculated, and difference spectra. Here, - represents the target phase $\left(\mathrm{Zn}_{2} \mathrm{Te}_{3} \mathrm{O}_{8}, \mathrm{PDF} \# 89-4454\right)$ at 97.08 mass $\%$ and $\triangle$ represents a minor impurity phase, $\mathrm{ZnTeO}_{3}(\mathrm{PDF} \# 75-1410)$ at 2.92 mass\% (Table 4.21).

Figure 4.49. Rietveld refinement of XRD spectra for the $\mathrm{SnTe}_{3} \mathrm{O}_{8}$ (left) and $\mathrm{TiTe}_{3} \mathrm{O}_{8}$ (right) samples after various heat treatments. The samples were run sequentially from the bottom spectra toward the top spectra. Here, represents the target phases of $\mathrm{SnTe}_{3} \mathrm{O}_{8}$ (PDF\#70-2440) and $\mathrm{TiTe}_{3} \mathrm{O}_{8}$ (PDF\#70-2439), $\triangle$ represents $\alpha-\mathrm{TeO}_{2}(\mathrm{PDF} 442-1365)$, and $\diamond$ represents the metal oxides of $\mathrm{SnO}_{2}(\mathrm{PDF} \# 70-6153)$ and $\mathrm{TiO}_{2}(\mathrm{PDF} \# 70-7347)$ in the corresponding figures (right and left, respectively) (see Table 4.21). The quantities of the constituents of these samples after each heat treatment are presented in Table 4.22 .

Figure 4.50. Rietveld refinement of $\mathrm{XRD}$ spectra for $\mathrm{ZrTe}_{3} \mathrm{O}_{8}$ after the $950{ }^{\circ} \mathrm{C}$ heat treatment. Here, - represents the target phase $\left(\mathrm{ZrTe}_{3} \mathrm{O}_{8}, \mathrm{PDF} \# 70-2442\right), \triangle$ represents $\alpha-\mathrm{TeO}_{2}$ (PDF\#42-1365), and $\diamond$ represents $\mathrm{ZrO}_{2}$ (PDF\#89-9069). Here, the $\mathrm{ZrTe}_{3} \mathrm{O}_{8}$ phase represents 97.21 mass $\%$ and $\mathrm{TeO}_{2}$ represents 2.79 mass $\%$ of the crystalline content.

Figure A.1. $\mathrm{TeO}_{4}$ trigonal bipyramidal, $t b p$, and $\mathrm{TeO}_{3}$ trigonal pyramidal, $t p$, structural units in tellurite glasses based on representations in the literature. Species presented in order of decreasing quantity of bridging oxygens $(4 \rightarrow 0)$. Oxygen assignments are broken down into equatorial positions $\left(\mathrm{O}_{\mathrm{eq}}\right)$ and axial positions $\left(\mathrm{O}_{\mathrm{ax}}\right)$ for $t b p 1$. Similar assignments could be made for $t b p 2$ and $t b p 3$. One oxygen of $\mathrm{TeO}_{3+1}$ is unique as it is farther away. Lone pair electrons are shown as small black dots near the central dark Te atom. Non-bridging oxygen (denoted as negative sign in parenthesis) assignments were chosen arbitrarily. This illustration is not exhaustive but shows the complexity of Te-O conformations.

\section{TABLES}

Table 2.1. Calculated composition of spent electrochemical salt with 20 mass $\% \mathrm{FP}\left(\mathrm{XCl}_{20}\right)$ (Yoo, 2009).

Table 2.2. Elemental composition of proposed spent electrochemical salt containing 20 mass $\%$ fission products, denoted as $X \mathrm{Cl}_{20}$. Also presented are the ionic radii (in picometers) for the $\mathrm{XCl}_{20}$ components (as well as $\mathrm{Te}^{4+}$ and $\mathrm{Pb}^{2+}$ for the tellurite glasses) along with the coordination number $(C N)$ of these ions (Shannon, 1976). 6

Table 2.3. Naturally occurring minerals investigated as potential waste forms (Mineralogy Database, 2011). The theoretical waste loading limits (in mass\%) for each mineral type based on $\mathrm{Cl}$ or alkali (on $\mathrm{Na}$ basis) are listed in parentheses and are adjusted based on the fraction of the alkali in the waste versus the fraction coming from the reactants (e.g., $\mathrm{NaAlO}_{2}$ ).

Table 2.4. Target elemental compositions of sodalite formulations (in molar formula units), glass sintering aids, and waste loading (mass \% addition to original composition). The "Label" is used to identify the samples in later sections. "Ln" denotes the addition of 
lanthanides. Here, "CS" is colloidal silica, "TEOS" is tetraethyl orthosilicate, and "GeEtO" is germanium(IV) ethoxide.. 8

Table 2.5. Elemental composition of proposed spent electrochemical salt containing 20 mass\% fission products. 8

Table 2.6. Summary results for the P-series tellurite glasses that were analyzed with the PCT. The "Glass" column lists those glasses batched on a 20-g scale for the PCT. Batch composition of each glass is listed in mass\% additive; the balance is $\mathrm{TeO}_{2}$. The composition of $X \mathrm{Cl}_{20}$ is found in Table 2.1. The melting temperature is denoted $T_{\mathrm{M}}$. The "volatility" is ranked based on degree of "fuming" observed during the melt process, from none (0) to a lot (4). Viscosity $(\eta)$ was estimated as compared to standards. The overall deviation associated with the density measurements is $\pm 0.015 \times 10^{-3} \mathrm{~kg} / \mathrm{m}^{3}$ (Riley et al., 2010). Phase separation was determined based on visual ("Bulk") and microscopy ("Small") observations. The normalized sodium release during the PCT is given as $\left(N L_{\mathrm{Na}}\right)$.

Table 3.1. Labels for the various sets of high-halide minerals fabricated in FY2011. Here, CS, TEOS, and Ge-EtO denote that samples were made with colloidal silica, tetraethyl orthosilicate, and germanium(IV) ethoxide, respectively. See Table 2.4 for the S5A and S6A compositions.

Table 3.2. Compositions (in mass\%) of glass sintering aid (SA) additions tested in FY2011. The NBS-1 (Vienna et al., 1998) glass from FY2010 studies is listed for comparison purposes (Riley et al., 2010). Also, some properties are listed and include the temperature at which the viscosity is $100 \mathrm{~Pa} \cdot \mathrm{s}$, the melting temperature $\left(T_{\mathrm{M}}\right)$, and the bulk density measured with a helium pycnometer. Some of the data was not available for $\mathrm{TePbO}\left(\mathrm{TPb}_{0}\right)$ or NaAlP-B4 for comparison, e.g., $T_{\mathrm{L}}$ represents the liquidus temperature, in place of the $T_{\mathrm{M}}$ for NaAlP-B4.

Table 3.3. Sample name designations for solution-derived sodalites in FY12

Table 3.4. Composition of the $\mathrm{XCl}_{20}$ salt added to the tellurite glasses along with the melting temperatures for some of the components (in order of mass fraction from largest to smallest). The melting temperature $\left(T_{\mathrm{M}}\right)$ and bulk density $\left(\rho_{\mathrm{b}}\right)$ are also included for each (Haynes, 2011).

Table 3.5. Compositions (in mole\%) from tellurite glass compositional variation study batched with and without $X \mathrm{Cl}_{20}$ additions. ${ }^{*}$ These values are also presented in mass $\%$ in our earlier work (Riley et al., 2010), although they are presented here as mole\% because that is more appropriate for the discussion of the glass structure. Each of the "+Cl" glasses were batched with a target of 10 mass $\% \mathrm{XCl}_{20}$-loading.

Table 3.6. Compositions of lead-tellurite variation study glasses in both mass $\%$ and mole $\%$. The subscripts on the glass identification (ID) represent the $X \mathrm{Cl}_{20}$-loading in mass $\% . \mathrm{TPb}_{0}$ and $\mathrm{TPb}_{10}$ represent compositions similar to $\mathrm{P}-8$ and $\mathrm{P}-11$, respectively, from previous work (Riley et al., 2010) but these glasses were all melted at $700{ }^{\circ} \mathrm{C}$ and with a different target mass ( $30 \mathrm{~g}$ vs. $20 \mathrm{~g}$ ). The sequential glass numbering identification, or "Alternate ID", is also provided for tracking purposes.

Table 3.7. Compositions for $\mathrm{T} 2 \mathrm{G}-21$, $\mathrm{T} 2 \mathrm{G}-21 \mathrm{~b}, \mathrm{~T} 2 \mathrm{G}-21 \mathrm{c}$, and $\mathrm{T} 2 \mathrm{G}-21 \mathrm{~d}$ (in mole $\%$ ) in which the mass $\%$ of $\mathrm{PbO}$ was $22,20,18$, and 16 mass $\%$, respectively. The baseline glass for this series is T2G-21, listed in bold type.

Table 3.8. Composition of $\mathrm{PbCl}_{2}-\mathrm{PbO}$ compositional variation glasses to study the structural effect of the anion in the lead-tellurite glasses. For all of the glasses in this series, the 
molar ratio, $M$, of $\mathrm{Pb}$ :Te was fixed at 0.202 . The baseline glass, $\mathrm{T} 2 \mathrm{G}-21$, is listed in bold type for reference.

Table 3.9. Summary of compositions for the waste-component-anion substitution glasses as well as the composition of $X \mathrm{O}_{20}$ with values sorted by mole\%. T2G-34 was fabricated from oxide versions of the chloride waste components in which the same total waste component cations (Cat.) were identical to the equivalent glass batched with the chloride components, T2G-22, thus T2G-22 serves as a reference for this study. Thus, the ratios of Te:Cat and $\mathrm{Pb}$ :Cat were fixed at 6.16 and 1.24, respectively. As with the other lead-tellurite glasses, the $\mathrm{Pb}$ :Te ratio for these glasses was fixed at 0.202 .

Table 3.10. Compositions for T2G-27a, T2G-27b, T2G-29a, and T2G-29b anion variation glasses (in mole\%) as well as the mass ratio of $\mathrm{TeO}_{2}: \mathrm{PbO}$. Here, $m$ and $M$ denote ratios by mass and moles, respectively. The baseline P-series glasses for this variation study, P-8 and P-4, are listed in bold type.....

Table 3.11. Compositions for $\mathrm{T} 2 \mathrm{G}-29 \mathrm{a}$ and $\mathrm{T} 2 \mathrm{G}-29 \mathrm{~b}$ anion variation glasses (in mole\%) as well as the mass ratio of $\mathrm{TeO}_{2}: \mathrm{PbO}$. Here, $m$ and $M$ denote ratios by mass and moles, respectively. The baseline P-series glasses for this variation study, P-8 and P-4, are listed in bold type.

Table 3.12. Tellurite crystals selected for fabrication at PNNL from the list proposed by Sakida et al. (1999a). Multiple steps are numbered for some of the crystals. Crystals in bold and italics show similar amorphous structure for the $\mathrm{TeO}_{3}$-isolated and $\alpha-\mathrm{TeO}_{2}$ units, respectively (see Figure 3.2). The methods listed here were those that proved successful by Sakida et al. "HT" stands for heat-treatment. Note: All crystals listed here also have an additional $0.3 \mathrm{~mol} \% \mathrm{Fe}_{2} \mathrm{O}_{3}$ added to shorten the relaxation time for the Te nuclei with $I=1 / 2$ during NMR analysis.

Table 3.13. Raman band assignments for tellurite glasses. Here, "tbp" denotes trigonal bipyramidal $\mathrm{TeO}_{4}$ units, " $t p$ " denotes trigonal pyramidal $\mathrm{TeO}_{3}$ units, "NBO" denotes a non-bridging oxygen, LFE denotes the low-frequency $(v)$ envelope, and HFE denotes the high-frequency envelope. The "Fitted" column denotes the peak locations for the fixed-frequency fitting technique that we used to fit the lead-tellurite and structural variations study glasses. The "E1" and "E2" bands were split from the "E" band for the lead tellurite and structural variation study glasses. The bands listed below "E2" are bands that were unique to a particular family of glasses and remain without a particular band name assignment due to their unique occurrences.

Table 4.1. Summary of phases identified in the halide minerals. The table shows powder diffraction file (PDF) and International Crystal Structure Database (ICSD) identifications, the space group (SG), the space group number (SG\#), and references for the patterns. More details can be found in our earlier work (Riley et al., 2011).

Table 4.2. Summary of sodalite mass $\%$, $\%$ porosity, calculated $\rho_{\mathrm{t}}$, and $\%$ of $\rho_{\mathrm{t}}$ for specimens containing 5 mass $\%$ additions of various sintering aids and fired at different temperatures. The maxima for sodalite and $\%$ of $\rho_{\mathrm{t}}$ are shown in bold type.

Table 4.3. Summary of solution $\mathrm{pH}$ results during sample preparation. Each value is listed as an additive value where the solution contains all of the contents listed above that additive.

Table 4.4. Density measurements for the specimens that were heated with spark plasma sintering. The data in parenthesis shows the percent of theoretical density for sodalite $\left(2.27 \times 10^{3} \mathrm{~kg} / \mathrm{m}^{3}\right)$...

Table 4.5. Phase distribution from XRD measurements. 
Table 4.6. Summary of characterizations for PCT specimens prior to the PCT. 47

Table 4.7. Averaged $N L_{\mathrm{i}}\left(\mathrm{g} / \mathrm{m}^{2}\right)$ in leachates from PCT-B with standard deviations listed in parenthesis. * Ln denotes total lanthanides analyzed and includes $\mathrm{Ce}, \mathrm{La}, \mathrm{Nd}, \mathrm{Pr}$, and $\mathrm{Sm}$. Note that the release values for Ba were below detection limits for SM-1 and SM2 .

Table 4.8. Summary $N L_{\mathrm{i}}\left(\mathrm{m}^{2} / \mathrm{g}\right)$ values of rinsate analysis. 48

Table 4.9. $N L_{\mathrm{i}}$ values $\left(\times 10^{-3} \mathrm{~g} / \mathrm{m}^{2}\right)$ adjusted for measured specific surface areas (standard deviations are below the average values). 48

Table 4.10. Summary of phases identified in the tellurite glasses for the composition-variation study.

Table 4.11. Results of quantitative fitting of Raman bands on P-series glasses. This table consists of peak location in wavenumber $\left(v, \pm 1 \mathrm{~cm}^{-1}\right)$ and integrated intensity, $I$. Note that these glasses were fitted with peak frequencies allowed to float (not fixed).....

Table 4.12. Ratios of key tellurite peaks from the Raman data. Since "B" was only observed in the Pb-containing glasses, the ratio $I(\mathbf{A}+\mathbf{B}) / I(\mathbf{C})$ is only different from $I(\mathbf{A}) / I(\mathbf{C})$ for these glasses.

Table 4.13. Summary of normalized release results from seven-day PCT-B for P-series tellurite glasses with $X \mathrm{Cl}_{20}$. More details are provided in our earlier work such as ranges/deviations (Riley et al., 2010).

Table 4.14. Summary of DTA-TGA results for P-8 and P-11 run at both 10 and $15{ }^{\circ} \mathrm{C} / \mathrm{min}$ ramp heating rates.

Table 4.15. Fitted Raman data for lead-tellurite and some of the structural variation study glasses. The band names are listed in bold type. Note that the frequencies were fixed during the fitting and are shown at the top of each column of integrated intensities.

Table 4.16. Quantitative Raman analysis of some of the lead-tellurite and structural variation glasses.

Table 4.17. Summary of crystalline compounds identified in lead-tellurite glasses with XRD.

Table 4.18. Summary of the DSC results for the lead-tellurite glasses. Variable headings are defined in the paragraph above.

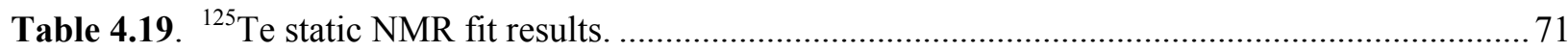

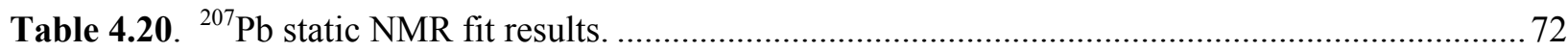

Table 4.21. Summary of crystalline phases identified in tellurite crystals. .75

Table 4.22. Mass\% of components in $\mathrm{SnTe}_{3} \mathrm{O}_{8}$ and $\mathrm{TiTe}_{3} \mathrm{O}_{8}$ samples after Rietveld refinements. The phases correspond to phases in Table 4.21 with graphical representations in Figure 4.49. The temperatures correspond to the heat treatment temperatures.

Table A.1. Tellurium oxide structural units present in glass according to the literature, represented in various notations 


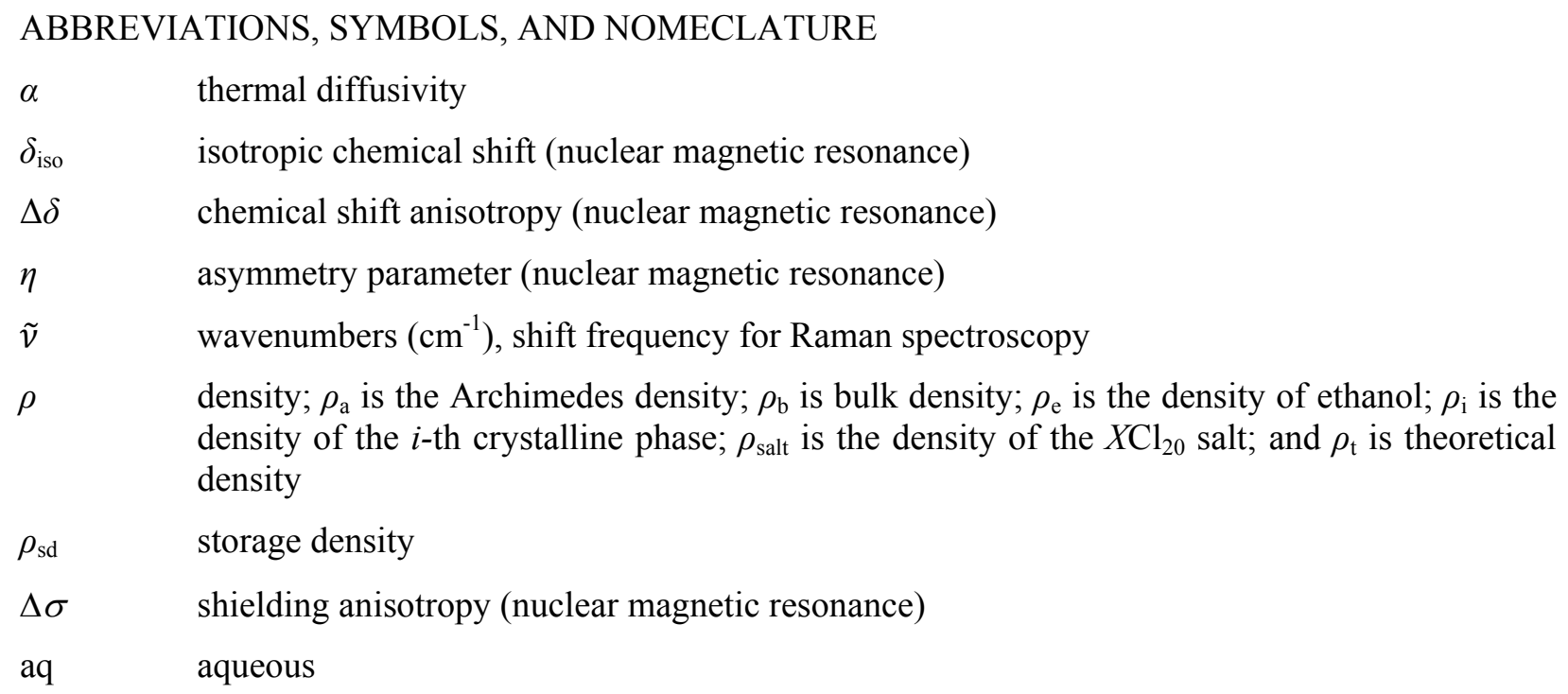

ASTM American Society for Testing and Materials, now ASTM International, Inc.

ATEM analytical transmission electron microscopy

BET Brunauer-Emmett-Teller

BF bright field

BSE backscattered electron (SEM imaging mode)

Cat. cation

CS colloidal silica

DOE U.S. Department of Energy

DF dark field

DSC differential scanning calorimetry or differential scanning calorimeter

DTA differential thermal analysis or differential thermal analyzer

Echem electrochemical separations process

EDS energy dispersive spectroscopy

EMSL Environmental Molecular Sciences Laboratory

$\mathrm{EtOH} \quad$ ethanol

FP fission products

FTIR Fourier transform infrared spectroscopy

FY fiscal year

Ge-EtO germanium(IV) ethoxide, $\mathrm{Ge}\left(\mathrm{OC}_{2} \mathrm{H}_{5}\right)_{4}$

HFE high-frequency envelope

HT heat-treatment

ICSD Inorganic Crystal Structure Database

ISP isostatic press

$K_{\mathrm{gl}} \quad$ glass formation constant 


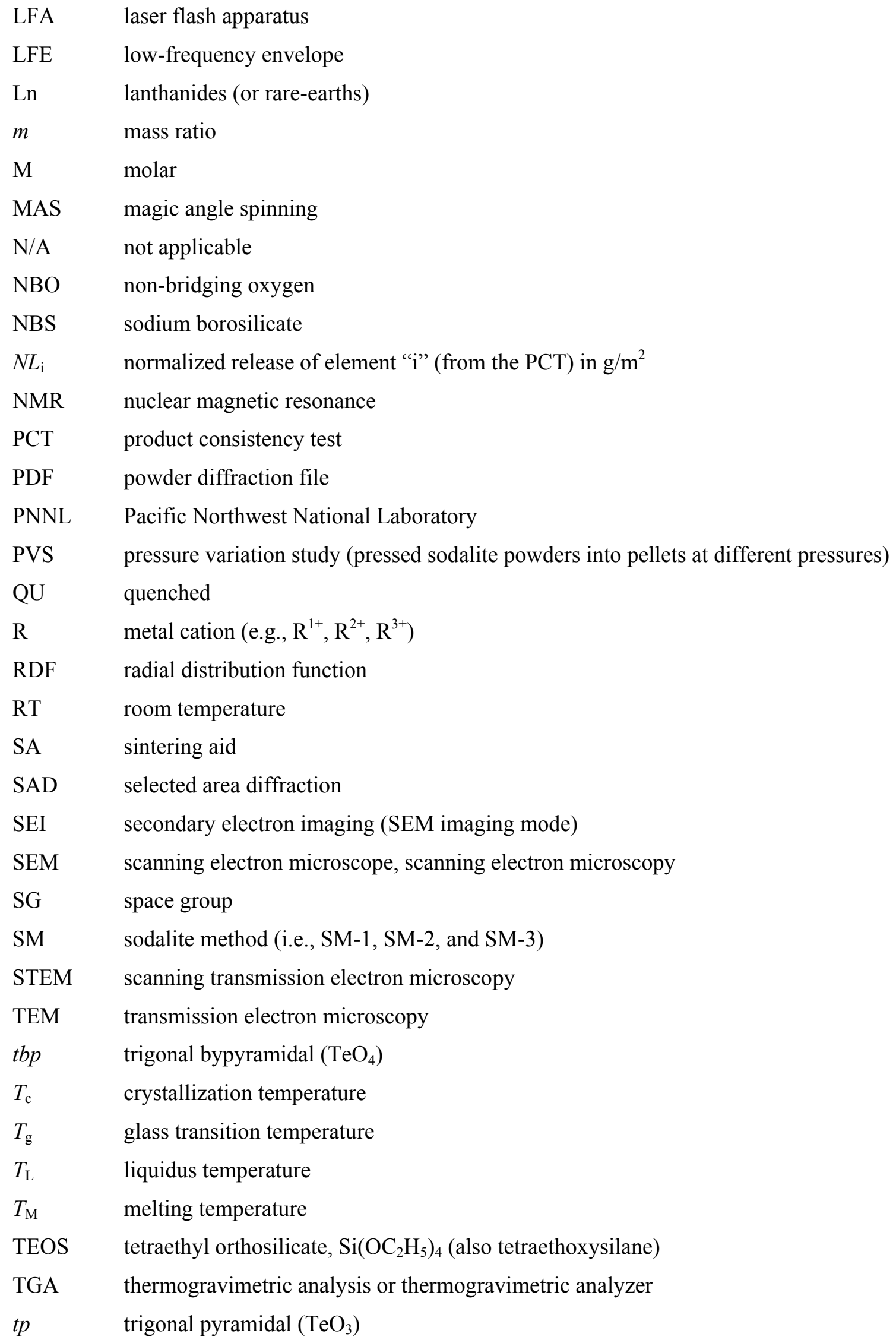


$\mathrm{TPb}_{\#} \quad$ lead tellurite glass where “\#” denotes the mass \% of added $X \mathrm{Cl}_{20}$

$T_{\mathrm{x}} \quad$ temperature of the crystallization onset

UAP uniaxial press

$X \mathrm{Cl}_{20} \quad$ mixed chloride electrochemical waste stream with $20 \% \mathrm{FP}$

XRD X-ray diffraction, X-ray diffractometer 



\section{INTRODUCTION}

An increased reliance on nuclear power is a likely solution to meet the clean-energy demands of the future (Vienna, 2010). The U.S. Department of Energy's (DOE's) Office of Nuclear Energy Fuel Cycle Research and Development (FCRD) Program is currently in the process of identifying the most efficient pathway for nuclear power production, which will likely involve a series of steps in which the fuel will be reprocessed and the reusable fuel recycled to recover fuel value. The waste from fuel processing will be immobilized in one of several different waste forms. A brief overview of the various types of proposed processing steps and waste streams is presented in our previous work (Riley et al., 2010). One of the separations methods being considered is an electrochemical process. In this method, the fuel is dissolved in molten ionic salts or a LiCl-KCl eutectic, and the resulting "spent salt" will need to be immobilized in a viable waste form. This spent salt is commonly referred to the "Echem salt" and consists primarily of an alkali chloride salt with minor amounts of alkaline earth, transition metal, lanthanide, and actinide chlorides plus a very small fraction of iodide.

The currently assumed waste form for immobilizing this salt waste is a glass-bonded sodalite, nominally $\mathrm{Na}_{4} \mathrm{Al}_{3}\left(\mathrm{SiO}_{4}\right)_{3} \mathrm{Cl}$ but often written as $\mathrm{Na}_{8}\left(\mathrm{AlSiO}_{4}\right)_{6} \mathrm{Cl}_{2}$ (Bateman et al., 2007; Ebert, 2005). This baseline waste form is fabricated by mixing zeolite $4 \mathrm{~A}$ with the salt waste. The salt is occluded in the zeolite at about $500{ }^{\circ} \mathrm{C}$; then the salt containing zeolite is mixed with borosilicate glass in a $3: 1$ mass ratio. The mixture is transferred to a mold and consolidated at $\sim 915^{\circ} \mathrm{C}$ with only slight pressure (a steel plate resting on top). The loading of waste in the zeolite is given by the reaction in Equation (1) below. After the 3:1 blending with borosilicate glass, the loading of fission-product-loaded salt in the final waste form is roughly 7.5 mass $\%$.

$$
\begin{aligned}
& \mathrm{Na}_{12}\left(\mathrm{AlSiO}_{4}\right)_{12}+4 \mathrm{NaCl} \rightarrow 2 \mathrm{Na}_{8}\left(\mathrm{AlSiO}_{4}\right)_{6} \mathrm{Cl}_{2} \\
& \text { (zeolite 4A) (halite) (sodalite) }
\end{aligned}
$$

The FCRD program has been supporting the development of alternative waste forms for the Echem salt waste since 2009. Part of that work has been at Pacific Northwest National Laboratory (PNNL), where we are developing a rare type of glass called a tellurite glass and high-halide minerals made with a solution-based process (Crum et al., 2009; Riley et al., 2010).

Tellurite glass has many attractive properties for a waste form, including high halide solubility over a wide range of different and mixed halides, high density (improving volumetric waste loading capacity), and good glass formation with chlorides and many other additives (Vogel et al., 1974; Yakhkind and Chebotarev, 1980). One of the primary drawbacks with these glasses is that they cost more to make than a traditional borosilicate-based glass waste form. Another drawback to these glasses is that since they have not previously been developed for waste form applications, many of the relevant engineering properties are unknown. In our earlier work, we discussed scoping tests for candidate waste form selection and the evaluation of a series of different tellurite glass systems for chemical durability, solubility of the salt waste, and glass properties (Riley et al., 2010). A few of the formulations showed good chemical durability and high waste loading capacity. The work presented here is a continuation of that work along with some additional fabrication and characterization data.

Our work has primarily focused on sodalite. The sodalite structure was identified as one of the naturally occurring structures that contain the waste components, i.e., alkalis and halides. These minerals have been under research for the past several years for incorporating the Echem salt components for the baseline waste form (Bateman et al., 2007; Ebert, 2005) as well as for radioactive iodine (Babad and Strachan, 1980; Koyama, 1994). However, we identified the solution-based process as a potential improvement to the preparation of the sodalites. The solution-based approach was identified because it allows for a near-room temperature aqueous processing route, reducing energy costs. It also allows for complete containment of the waste in a solution versus processing the salt as a powder that can become airborne. The sodalite powders that were formed upon drying were small $(\sim 1-5 \mu \mathrm{m})$ and thus needed to 
be sintered together to form a viable waste form. However, this firing process only yielded pellets that were $\sim 70 \%$ dense compared to the theoretical density of sodalite, $2.27 \times 10^{3} \mathrm{~kg} / \mathrm{m}^{3}$. Therefore, a sintering aid was added to further densify the pellets. Small additions ( 2 mass\%) of a high-viscosity glass, NBS-1, dramatically increased the density of the pellets to $>90 \%$ of theoretical density (Vienna et al., 1998).

In FY2010, we assessed the chemical durability of these different waste forms with the Product Consistency Test (PCT) ${ }^{[1]}$ and some of the waste forms we made for each family of materials showed promise. Since both of these waste forms showed good performance in the PCT, they were both candidates for further consideration.

The chemical durability results for the tellurite glasses in FY2010 revealed a wide variety of waste form performance in these materials. The most promising glass studied was a lead-tellurite glass with the Echem salt simulant. This glass showed very good chemical durability: the normalized release for sodium was $0.478 \mathrm{~g} / \mathrm{m}^{2}$ and comparable to borosilicate glasses (Ebert and Wolf, 1999). With this in mind, the following studies were undertaken in FY2011 to further investigate and optimize these glasses as a potential waste form:

1) Expanded study on lead-tellurite glasses. The lead-tellurite glass system was further investigated to determine the waste-loading limits and assess the tellurium-oxygen bonding structure as a function of waste loading by characterization with nuclear magnetic resonance, Raman spectroscopy, and X-ray diffraction (XRD). The Te-O network in tellurite glass is quite complex where at least eight different structures have been presented in the literature and typically include $\mathrm{TeO}_{4}, \mathrm{TeO}_{3+1}$, and $\mathrm{TeO}_{3}$ structures present in either a trigonal bipyramidal or trigonal pyramidal arrangement. The structural investigation revealed that the medium-range order in the glass decreased and the tellurite network was depolymerized as the waste fraction was increased. It was apparent that glass-network breaking was predominantly caused by the additions of the waste cations and that the presence of chloride may have actually caused some repolymerization of the network by scavenging alkali and preventing it from acting as a network modifier. At the highest waste loadings, the glass became phase-separated, resulting in a Te-rich phase and a $\mathrm{Pb}$-alkali-chloride-rich phase, and some chloride-containing crystals were formed. We also investigated some of the other waste-form related properties of the leadtellurite glasses, such as the thermal diffusivity, glass transition temperature, crystallization temperature, melting temperature, and mass loss as a function of temperature.

2) Investigated the glass network structure of P-series glasses from FY2010 with Raman spectroscopy. Here, we studied the glass structure of the P-series glasses $\left(\mathrm{TeO}_{2}\right.$ with various other oxide additions such as $\mathrm{ZnO}, \mathrm{PbO}, \mathrm{P}_{2} \mathrm{O}_{5}, \mathrm{Al}_{2} \mathrm{O}_{3}, \mathrm{~B}_{2} \mathrm{O}_{3}$, and $\mathrm{WO}_{3}$ ); the study provided a preliminary understanding of the various Te-O structural configurations in the literature and how to identify them with Raman spectroscopy. The goal was to correlate a particular Te-O structure with a glass that had high chemical durability and engineer a glass with very high chemical durability by targeting the preferred structure with changes in composition. This has proved unsuccessful overall, but we did observe some unique frequency bands in the Raman spectra for the lead-tellurite glasses that might be a first step toward better understanding the link between the glass structure and the chemical durability.

3) Investigated the structural effects of various components in the tellurite glasses. For this study, we used Raman spectroscopy to look at the structure of simplified glasses in which various components had been removed and added to provide different combinations of the anions and cations. This study provided some very useful information regarding the effects of $\mathrm{Cl}$ and alkali on the tellurite glass network.

[1] ASTM C 1285-08. 2008. Standard Test Methods for Determining Chemical Durability of Nuclear, Hazardous, and Mixed Waste Glasses and Multiphase Glass Ceramics: The Product Consistency Test (PCT), American Society for Testing and Materials International, West Conshohocken, PA. 
4) Investigated tellurite crystals as a surrogate for tellurite glass. In addition to the tellurite glasses, we also looked at some tellurite crystals. The goal of this study was to look at the chemical durability of tellurite compounds that contained only one structural configuration of Te-O. This idea was proposed based on results presented by Sakida et al. (Sakida et al., 1999a). Sakida et al. prepared 21 tellurite crystals, characterized them with ${ }^{125} \mathrm{Te}$ nuclear magnetic resonance (NMR), and then categorized them based on the structural configuration of the Te present in the crystals (see Figure 1.1). Each of these crystals fell into a particular category defined as $\mathrm{TeO}_{3}, \mathrm{TeO}_{3+1}$, or $\mathrm{TeO}_{4}$, where $\mathrm{TeO}_{4}$ structural units were divided into $\alpha-\mathrm{TeO}_{2}$ and $\beta-\mathrm{TeO}_{2}$. Single-phase tellurite crystals have a single type of tellurium-oxygen structure in contrast with tellurite glasses that have a mixed set of most of the different types of structures. The goal was to make a series of tellurite crystals from each category and assess the chemical durability of each. In FY2011, we made a series of crystals, some of which were highly phase pure $(>95 \%)$. We intend to verify their structure (with NMR and/or Raman spectroscopy) and perform chemical durability tests on them to look for a correlation between the structure and the chemical durability. We wanted to achieve melting so that the chunks could be ground and sieved and a particular size fraction could be retained for chemical durability tests. We had difficulty melting all of the mixtures that we attempted.

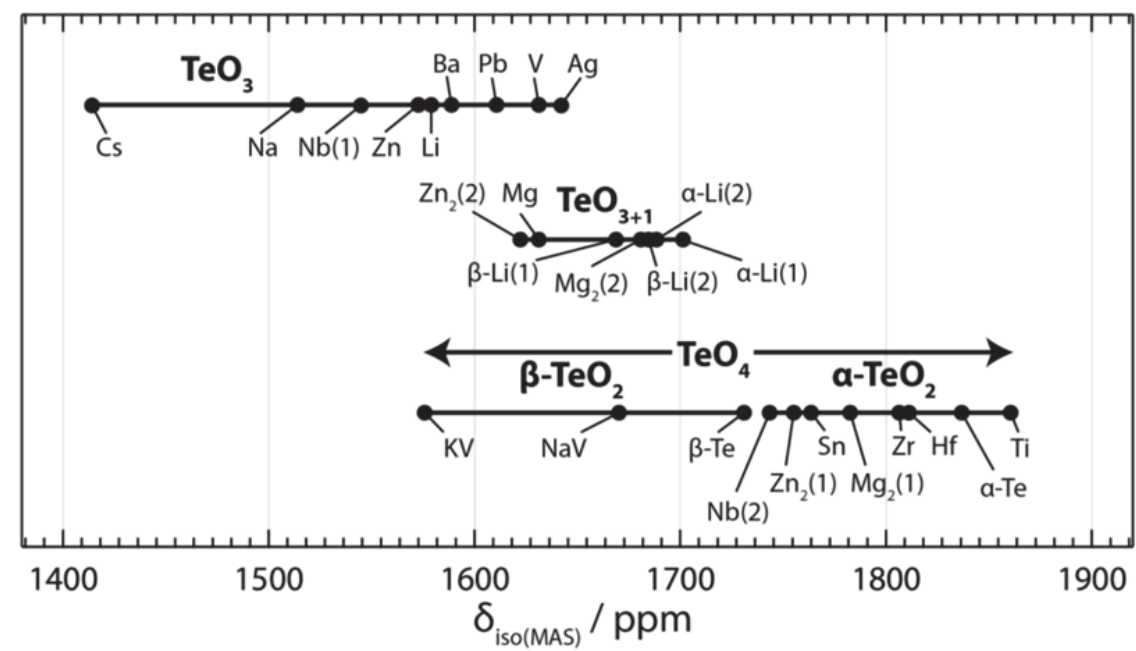

Figure 1.1. Ranges of isotropic chemical shifts $\left(\delta_{\text {iso }}\right)$ for tellurium sites with different structural units determined with ${ }^{125}$ Te magic angle spinning (MAS) NMR at $9.4 \mathrm{~T}$ - recreated from Sakida et al. (1999a).

For the second candidate waste form, the high-halide minerals, we looked at optimizing a few of the variables in the solution-based process as well as some post-process adjustments to the heat-treatments. These variable studies included the following:

1) Alternative silica sources. We fabricated solution-derived sodalites with an organic source of silica, tetraethyl orthosilicate (TEOS), in place of the colloidal silica (CS) (see Figure 1.2a). The goal of this substitution was to assess the differences in reaction rate for sodalite formation between the silica sources as well as the differences in sodalite concentration. The change in silica sources resulted in a noticeable improvement, from 23.8 mass\% sodalite in the S5A specimen with CS to 95.7 mass\% sodalite in the S5C specimen with TEOS.

2) Investigated alternative 4-coordinated network formers. We performed the solution-based process with germanium (IV) ethoxide, an organic form of $\mathrm{Ge}^{4+}$, in place of the TEOS to see whether we could fabricate a germanium-based sodalite (see Figure 1.2b). The germanium sodalite was targeted because it was predicted to (1) reduce the firing temperature resulting in an increased sodalite concentration because of the reduced chloride volatility (due to lower firing temperature) and (2) increase fired-pellet density improving volumetric waste loading. The high porosity in the initially fired pellets was a deterrent to pursuing this route any further, though we did demonstrate a high 
germania-based sodalite concentration ( 82 mass \%) in the as-made gel. The high porosity in conjunction with the high cost of germanium (IV) ethoxide led us to abandon this pursuit.

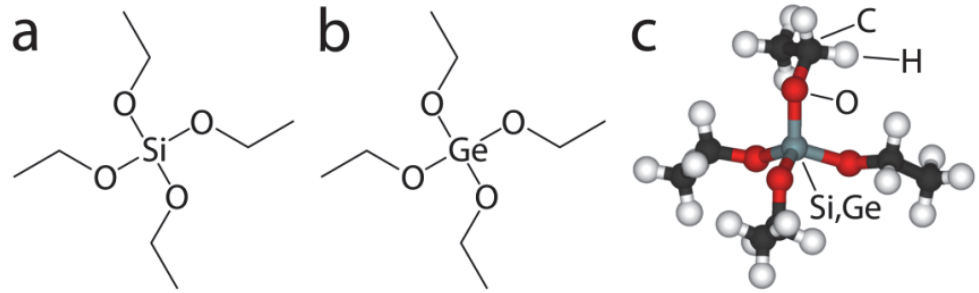

Figure 1.2. (a) Hydrocarbon stick figure of TEOS, (b) hydrocarbon stick figure of germanium(IV) ethoxide, and (c) a three-dimensional view of these structures. Note: for (a) and (b), the atom located at each bend and terminal is a $C$ atom where each $C$ has four total bonds achieved with hydrogen atoms in areas where oxygen is not present.

3) Alternative sintering aids. We had success in FY2010 with the NBS-1 glass, but due to the high viscosity of this glass, the temperatures required for pellet densification were rather high $\left(850{ }^{\circ} \mathrm{C}\right)$ and that led to chloride volatility, evidenced by a decrease in the sodalite concentration (Vienna et al., 1998). We fabricated a series of six alternative sintering aids with different viscosities in order to consolidate the solution-derived powders at a lower temperature, retaining the sodalite structure and preventing chloride volatility. These sintering aids included four sodium borosilicate glasses, a sodium aluminophosphate glass, and a lead-tellurite glass. Each of these glasses was added to a separate batch, as a powder; the gel was dried, specimens from the batches were pressed into pellets, fired at different temperatures, and the phases quantified with XRD. The sodium borosilicate glasses dramatically improved the densification (up to $96 \%$ of theoretical density) of the fired pellets at much-reduced temperatures of $\sim 750{ }^{\circ} \mathrm{C}$, where rather large amounts of sodalite were still produced (70.6 mass \%).

4) Alternative approaches to making sodalite. In FY2012, we took a different approach to making high density sodalite pellets. Three different solution-based approaches were evaluated to make sodalite with the same simulated $X_{C_{20}}$ salt. Several aspects were varied during the process including: the reactants, the amount of sintering aid added $(0,5$, and 10 mass $\%)$, the step in the process when the sintering aid was added, calcination, and the timing of the calcination step in the process. The three sodalite methods ( $S M$-\#) used in the current study are $S M-1, S M-2$, and $S M-3$, simplified below. The equations above are listed with the salt being denoted as " $\mathrm{NaCl}$ " but, in fact, the actual salt compositions contain $\mathrm{R}^{1+}, \mathrm{R}^{2+}$, and $\mathrm{R}^{3+}$ cations so the $\mathrm{R}: \mathrm{Cl}$ ratio is not $1: 1(\mathrm{R}: \mathrm{Cl}<1)$. The reactants were added in the proper amounts to obtain the targeted value for $\mathrm{Al}: \mathrm{Cl}(3: 1)$ and $\mathrm{Si}: \mathrm{Cl}(3: 1)$ where the alkali fraction $\left(\mathrm{R}^{1+}\right)$ was intentionally below the target of $4: 1\left(\mathrm{R}^{1+}: \mathrm{Cl}\right)$ to help influence the incorporation of alkaline earth $\left(\mathrm{R}^{2+}\right)$ and $\mathrm{Ln}\left(\mathrm{R}^{3+}\right)$ components from the $X \mathrm{Cl}_{20}$ into the sodalite structure. The results from this study showed that each formulation had advantages and disadvantages where SM-3 pellets had high density and low sodalite production and SM-2 pellets had very high sodalite production with modestly high densities. A mixture of these methods will likely be considered in FY2013.

SM-1: $\quad 6 \mathrm{NaOH}+6 \mathrm{Al}(\mathrm{OH})_{3}+2 \mathrm{NaCl}+6 \mathrm{SiO}_{2} \rightarrow \mathrm{Na}_{8}\left(\mathrm{AlSiO}_{4}\right)_{6} \mathrm{Cl}_{2}+24 \mathrm{OH}^{-}$

SM-2:

$6 \mathrm{NaAlO}_{2}+2 \mathrm{NaCl}+6 \mathrm{SiO}_{2} \rightarrow \mathrm{Na}_{8}\left(\mathrm{AlSiO}_{4}\right)_{6} \mathrm{Cl}_{2}$

SM-3:

$$
6 \mathrm{NaOH}+3 \mathrm{Al}_{2} \mathrm{Si}_{2} \mathrm{O}_{7}+2 \mathrm{NaCl} \rightarrow \mathrm{Na}_{8}\left(\mathrm{AlSiO}_{4}\right)_{6} \mathrm{Cl}_{2}+6 \mathrm{OH}^{-}
$$

\section{EXPANDED BACKGROUND AND APPROACH}

The simulated (nonradioactive) waste composition used in this study is the 20 mass $\%$ fission-products (FP) waste stream (Yoo, 2009) as presented in Table 2.1. The 20 mass\% FP Echem waste stream is 
designated in this report as $X \mathrm{Cl}_{20}$ and was discussed in more detail in our previous publications (Crum et al., 2009; Riley et al., 2010).

Table 2.1. Calculated composition of spent electrochemical salt with 20 mass $\% \mathrm{FP}\left(X \mathrm{Cl}_{20}\right)(\mathrm{Yoo}, 2009)$.

\begin{tabular}{lll}
\hline Element & Mole Fraction & Mass Fraction \\
\hline $\mathrm{Y}$ & 0.0007 & 0.0020 \\
$\mathrm{La}$ & 0.0029 & 0.0127 \\
$\mathrm{Ce}$ & 0.0045 & 0.0195 \\
$\mathrm{Pr}$ & 0.0022 & 0.0095 \\
$\mathrm{Nd}$ & 0.0067 & 0.0301 \\
$\mathrm{Pm}$ & 0.0003 & 0.0011 \\
$\mathrm{Sm}$ & 0.0020 & 0.0092 \\
$\mathrm{Eu}$ & 0.0002 & 0.0011 \\
$\mathrm{Gd}$ & 0.0002 & 0.0011 \\
$\mathrm{~Tb}$ & 0.0000 & 0.0001 \\
$\mathrm{Li}$ & 0.2219 & 0.0477 \\
$\mathrm{~K}$ & 0.1603 & 0.1942 \\
$\mathrm{Na}$ & 0.0630 & 0.0449 \\
$\mathrm{Rb}$ & 0.0006 & 0.0017 \\
$\mathrm{Cs}$ & 0.0081 & 0.0332 \\
$\mathrm{Sr}$ & 0.0013 & 0.0036 \\
$\mathrm{Ba}$ & 0.0030 & 0.0129 \\
$\mathrm{Cl}$ & 0.5212 & 0.5725 \\
Total & 1.0000 & 1.0000 \\
\hline Simplified & Mole Fraction & Mass fraction \\
\hline Alkali & 0.4539 & 0.3217 \\
Alkaline Earth & 0.0044 & 0.0166 \\
Lanthanide & 0.0198 & 0.0863 \\
Halide & 0.5219 & 0.5754 \\
\hline & &
\end{tabular}

This waste stream was simplified by combining minor components into major like components to allow for easier batching. This simplified waste stream along with some ionic radii from the literature are shown in Table 2.2 (Shannon, 1976). The changes included the following:

- $\mathrm{Y}, \mathrm{Tb}$, actinides (not shown due to low levels), and minor lanthanides (Pm, Gd, Eu) were grouped into the $\mathrm{Nd}$ component - the only lanthanides used in this study were $\mathrm{La}, \mathrm{Ce}, \mathrm{Pr}, \mathrm{Nd}$, and $\mathrm{Sm}$

- The $\mathrm{Rb}$ component was grouped in with the Li component

- Sr was grouped in with Ba 
Table 2.2. Elemental composition of proposed spent electrochemical salt containing 20 mass $\%$ fission products, denoted as $X \mathrm{Cl}_{20}$. Also presented are the ionic radii (in picometers) for the $X \mathrm{Cl}_{20}$ components (as well as $\mathrm{Te}^{4+}$ and $\mathrm{Pb}^{2+}$ for the tellurite glasses) along with the coordination number $(C N)$ of these ions (Shannon, 1976).

\begin{tabular}{|c|c|c|c|c|c|}
\hline \multirow{2}{*}{$\begin{array}{l}\text { Salt Waste } \\
\text { Component }\end{array}$} & \multicolumn{2}{|c|}{$X \mathrm{Cl}_{20}$} & \multicolumn{3}{|c|}{ Ionic radius, $r_{\text {ion }}$} \\
\hline & Mole\% & Mass \% & Cation & $r_{\text {ion }}(\mathrm{pm})$ & $C N$ \\
\hline $\mathrm{LiCl}$ & 46.82 & 29.89 & $\overline{\mathrm{Li}^{+}}$ & 59.0 & IV \\
\hline $\mathrm{KCl}$ & 33.83 & 37.99 & $\mathrm{~K}^{+}$ & 137 & IV \\
\hline $\mathrm{NaCl}$ & 13.30 & 11.71 & $\mathrm{Na}^{+}$ & 99 & IV \\
\hline $\mathrm{CsCl}$ & 1.54 & 3.91 & $\mathrm{Cs}^{+}$ & 167 & VI \\
\hline $\mathrm{NdCl}_{3}$ & 1.42 & 5.36 & $\mathrm{Nd}^{3+}$ & 98.3 & VI \\
\hline $\mathrm{CeCl}_{3}$ & 0.95 & 3.51 & $\mathrm{Ce}^{3+}$ & 101 & VI \\
\hline $\mathrm{BaCl}_{2}$ & 0.64 & 2.01 & $\mathrm{Ba}^{2+}$ & 135 & VI \\
\hline $\mathrm{LaCl}_{3}$ & 0.62 & 2.29 & $\mathrm{La}^{3+}$ & 103.2 & VI \\
\hline $\mathrm{PrCl}_{3}$ & 0.46 & 1.72 & $\operatorname{Pr}^{3+}$ & 99 & VI \\
\hline $\mathrm{SmCl}_{3}$ & 0.42 & 1.61 & $\mathrm{Sm}^{3+}$ & 95.8 & VI \\
\hline Total & 100.00 & 100.00 & $\mathrm{Cl}^{-}$ & 181 & VI \\
\hline \multicolumn{3}{|c|}{ Simplified (by Group) } & $\overline{\mathrm{Te}^{4+}}$ & 52 & III \\
\hline$\overline{\text { Alkali }}$ & 45.39 & 32.17 & $\mathrm{Te}^{4+}$ & 66 & IV \\
\hline Alkaline earth & 0.44 & 1.66 & $\mathrm{~Pb}^{2+}$ & 98 & IV \\
\hline Lanthanide & 1.98 & 8.63 & $\mathrm{~Pb}^{2+}$ & 119 & VI \\
\hline Halide & 52.19 & 57.54 & & & \\
\hline
\end{tabular}

\subsection{High-Halide Minerals: Background and Literature Survey}

It is not as easy to incorporate radionuclides into a ceramic waste form as it is to incorporate them into a glass, a more forgiving host network. The incorporation of waste radionuclides in a ceramic waste form is limited to two options, in which (1) a radionuclide is actually incorporated into the structure of the ceramic material at a specific atomic position in the periodic structure or (2) a radionuclide is captured by the non-radioactive phase (Ewing et al., 1995). In order for option (1) to be possible, the radionuclide properties must be suitable for incorporation into the ceramic crystal structure imposed by the coordination polyhedra, which include specific size, charge, and bonding constraints. Clearly, the requirements on a target ceramic waste would have to include the ability to accommodate a variety of atomic substitutions of varying sizes and charges to allow for charge balance in the structure. Considering option (2), a common approach has been to immobilize individual grains of radioactive phases in a ceramic phase such as $\mathrm{TiO}_{2}$ or $\mathrm{Al}_{2} \mathrm{O}_{3}$ that have high chemical durability.

To start this study, a literature survey was conducted to identify naturally occurring minerals that are high in halides and/or alkali oxides (see Table 2.3) (Crum et al., 2009). When comparing the candidate minerals listed in Table 2.3, pyrosmalite-(Mn) (formerly manganpyrosmalite) and brokenhillite both contain significant concentrations of $\mathrm{Cl}$; however, the lack of alkali compromises their ability to be good candidates for the spent salt. Wadalite (sodalite group) is a closer compositional match to the spent salt, but again the lack of alkali in this mineral means it is not the most ideal candidate for immobilizing the cation fraction of the waste. According to the list in Table 2.3, the best candidate materials for both the $\mathrm{Cl}^{-}$and the alkali are sodalite, apatite, and cancrinite (quadridavyne). 
Table 2.3. Naturally occurring minerals investigated as potential waste forms (Mineralogy Database, 2011). The theoretical waste loading limits (in mass\%) for each mineral type based on $\mathrm{Cl}$ or alkali (on $\mathrm{Na}$ basis) are listed in parentheses and are adjusted based on the fraction of the alkali in the waste versus the fraction coming from the reactants (e.g., $\mathrm{NaAlO}_{2}$ ).

\begin{tabular}{lllrr}
\hline Mineral Name & Mineral Group & Chemical Formula & \multicolumn{1}{c}{$\mathrm{Cl}$} & \multicolumn{1}{c}{ Alkali } \\
\hline Pyrosmalite-(Mn) & Pyrosmalite & $\left(\mathrm{Mn}, \mathrm{Fe}^{2+}\right)_{8} \mathrm{Si}_{6} \mathrm{O}_{15}(\mathrm{OH}, \mathrm{Cl})_{10}$ & $29.30(51)$ & $0.00(0)$ \\
Wadalite & Sodalite & $\mathrm{Ca}_{6} \mathrm{Al}_{5} \mathrm{Si}_{2} \mathrm{O}_{16} \mathrm{Cl}_{3}$ & $13.40(23)$ & $0.00(0)$ \\
Brokenhillite & Pyrosmalite & $(\mathrm{Mn}, \mathrm{Fe})_{32}\left(\mathrm{Si}_{24} \mathrm{O}_{60}\right) \mathrm{OH}_{29} \mathrm{Cl}_{11}$ & $9.06(16)$ & $0.00(0)$ \\
Quadridavyne & Cancrinite & $(\mathrm{Na}, \mathrm{K})_{6} \mathrm{Ca}_{2} \mathrm{Al}_{6} \mathrm{Si}_{6} \mathrm{O}_{24} \mathrm{Cl}_{4}$ & $13.20(23)$ & $12.84(27)$ \\
Chorapatite & Apatite & $(\mathrm{Na}, \mathrm{Ca})_{5}\left(\mathrm{PO}_{4}\right)_{3} \mathrm{Cl}$ & $8.14(14)$ & $26.41(16)$ \\
Sodalite & Sodalite & $\mathrm{Na}_{8} \mathrm{Al}_{6} \mathrm{Si}_{6} \mathrm{O}_{24} \mathrm{Cl}_{2}$ & $7.32(13)$ & $18.98(15)$ \\
\hline
\end{tabular}

Apatites follow the composition of $\mathrm{R}_{5}\left(\mathrm{YO}_{4}\right)_{3} \mathrm{X}$ where " $\mathrm{R}$ " can be any number of atoms, i.e., $\mathrm{R}^{1+}, \mathrm{R}^{2+}$ or $\mathrm{R}^{3+}$; "Y" (not yttrium) is $\mathrm{P}, \mathrm{Si}, \mathrm{V}$, or Os; and " $\mathrm{X}$ " is F, Cl, I, OH, or O (Dong et al., 2002; Park et al., 2002). This suggests a wide range of compositional flexibility in which $\mathrm{R}$ could be anything from alkalis $\left(\mathrm{R}^{1+}\right)$ to alkaline earths $\left(\mathrm{R}^{2+}\right)$ to lanthanides $\left(\mathrm{R}^{3+}\right)$, all of which are components of the waste, although the alkaline earths are the most commonly found cations to occupy these sites in naturally occurring apatites. Typical apatites found in nuclear waste solids are lanthanide $(\mathrm{Ln})$ silicate isomorphs of the natural apatite that have a composition of $\mathrm{Ca}_{4-x} \mathrm{Ln}_{6+x}\left(\mathrm{SiO}_{4}\right)_{6}\left(\mathrm{PO}_{4}\right)_{y} \mathrm{O}_{2}$ where the actinides can readily substitute for the lanthanides. Also, iodide is expected to be present in small fractions in the waste stream and could also be incorporated into an iodoapatite structure. Apatites have a few drawbacks. One is the low waste loading by mass (see Table 2.3). Another is their ability to become amorphous after radioactive decay that induces microcracking from volume expansion (Weber et al., 1979). A detailed presentation of these radiation-induced changes can be found elsewhere (Ewing et al., 1995). Vance and coworkers (2012) investigated apatites as a waste form for a similar waste and had problems getting the apatite structure to accommodate all of the waste cations. Apatites have not yet been studied at PNNL for this waste stream but will likely be studied in the future.

All of the work at PNNL has focused on sodalite and cancrinite mineral structures. Based on the literature, sodalite is more likely to form when the only anion present is $\mathrm{Cl}^{-}$. If significant concentrations of $\mathrm{CO}_{3}{ }^{2-}, \mathrm{NO}_{3}{ }^{-}$, or $\mathrm{OH}^{-}$are present, then cancrinite can become the preferred structure (Liu et al., 2007; Ocanto et al., 2008; Ocanto et al., 2009). In FY2010, we made changes to the solution chemistry in an attempt to form cancrinite in place of sodalite and we had limited success as presented in our previous work (Riley et al., 2010). The additions of $\mathrm{NO}_{3}{ }^{-}, \mathrm{CO}_{3}{ }^{2-}$, and $\mathrm{OH}^{-}$were examined to determine which, if any, would form cancrinite instead of sodalite, since cancrinite has a higher theoretical waste loading (23 mass\%) than sodalite (13 mass\%). The targeted compositions for sodalite synthesis are given in Table 2.4. The $X \mathrm{Cl}_{20}$ salt composition used in these experiments is presented in Table 2.5. Note: the method for cancrinite mineral synthesis is presented in our earlier work (Riley et al., 2010).

The FY2009 scoping studies showed that sodalite can be easily synthesized with a solution-based process (Crum et al., 2009). However, it is produced in the form of a fine powder with particle sizes on the order of a few microns; the small particle size produced by this technique makes it necessary to treat the powders to form a monolith. In this study, the powders were pressed into pellets (19-25 mm diameter, 5$10 \mathrm{~mm}$ thick) and fired to achieve the desired bulk density (>90\% of theoretical density). Sodalite was the predominant structure that formed during the solution-based process during the scoping studies of FY2009. Cancrinite, while identified as the best candidate mineral in terms of maximum waste loading, was produced during the process but converted to sodalite upon firing. 
Table 2.4. Target elemental compositions of sodalite formulations (in molar formula units), glass sintering aids, and waste loading (mass \% addition to original composition). The "Label" is used to identify the samples in later sections. "Ln" denotes the addition of lanthanides. Here, "CS" is colloidal silica, "TEOS" is tetraethyl orthosilicate, and "Ge-EtO" is germanium(IV) ethoxide.

\begin{tabular}{lccccc}
\hline $\begin{array}{l}\text { Label: } \\
\text { Component/Property }\end{array}$ & $\begin{array}{c}\text { Ideal } \\
\text { Sodalite }\end{array}$ & $\begin{array}{c}\text { S5A } \\
\text { Series }\end{array}$ & $\begin{array}{c}\text { S5C } \\
\text { Series }\end{array}$ & $\begin{array}{c}\text { S5D } \\
\text { Series }\end{array}$ & $\begin{array}{c}\text { S6 } \\
\text { Series }\end{array}$ \\
\hline Alkali & $\mathbf{8 . 0}$ & 7.9 & 7.9 & 7.9 & 7.9 \\
$\mathrm{Al}$ & $\mathbf{6 . 0}$ & 6.0 & 6.0 & 6.0 & 6.0 \\
$\mathrm{Si} / \mathrm{Ge}$ & $\mathbf{6 . 0}$ & 6.0 & 6.0 & 6.0 & 6.0 \\
$\mathrm{Si} / \mathrm{Ge}$ additive & - & $\mathrm{CS}$ & $\mathrm{TEOS}$ & Ge-EtO & $\mathrm{CS}$ \\
Alkaline earth + Ln & - & 0.1 & 0.1 & 0.1 & 0.1 \\
$\mathrm{Cl}^{-}, \mathrm{NO}_{3}^{-}$ & $\mathbf{2 . 0}$ & 2.0 & 2.0 & 2.0 & 2.0 \\
O & $\mathbf{2 4 . 0}$ & 24.0 & 24.0 & 24.0 & 24.0 \\
\hline Glass sintering aid, mass\% & - & - & $5^{*}$ & - & $1.0-7.0^{\dagger}$ \\
\hline Total & $\mathbf{4 6 . 0}$ & 46.0 & 46.0 & 46.0 & 46.0 \\
Waste loading, mass\% & - & 13.7 & 13.7 & 10.7 & $12.7-13.5$ \\
\hline
\end{tabular}

*Sintering aids listed Table 3.2 (other than NBS-1) were used. ${ }^{\dagger}$ NBS-1 glass was used.

Table 2.5. Elemental composition of proposed spent electrochemical salt containing 20 mass \% fission products.

\begin{tabular}{|c|c|c|c|}
\hline Component & Mass Fraction & Conc. $(\mathrm{g} / \mathrm{L})$ & $\mathrm{M}$ (moles/L) \\
\hline $\mathrm{KCl}$ & 0.3799 & 68.4327 & 0.9179 \\
\hline $\mathrm{LiCl}$ & 0.2985 & 53.7685 & 1.2683 \\
\hline $\mathrm{NaCl}$ & 0.1171 & 21.0941 & 0.3609 \\
\hline $\mathrm{NdCl}_{3}$ & 0.0537 & 9.6632 & 0.0386 \\
\hline $\mathrm{CsCl}$ & 0.0395 & 7.1180 & 0.0423 \\
\hline $\mathrm{CeCl}_{3}$ & 0.0352 & 6.3449 & 0.0257 \\
\hline $\mathrm{LaCl}_{3}$ & 0.0229 & 4.1324 & 0.0168 \\
\hline $\mathrm{BaCl}_{2}$ & 0.0202 & 3.6315 & 0.0174 \\
\hline $\mathrm{PrCl}_{3}$ & 0.0169 & 3.0455 & 0.0123 \\
\hline $\mathrm{SmCl}_{3}$ & 0.0160 & 2.8809 & 0.0112 \\
\hline Sum: & 1.0000 & 180.11 & 2.7116 \\
\hline
\end{tabular}

The FY2010 efforts focused on two areas that include 1) increasing the bulk density of the sodalite waste form and 2) synthesis of a cancrinite waste form (Riley et al., 2010). To increase bulk density of the sodalite, the firing temperature was varied to determine the maximum firing temperature above which the volatility of $\mathrm{Cl}$ became significant. First, the optimum firing temperature was determined based on bulk density, $\mathrm{Cl}$ loss, and PCT response. Second, a sintering aid (1-7 mass\%) was added in the form of powdered borosilicate glass (NBS-1, see Table 3.2) (Vienna et al., 1998). The powdered glass was added during the process, directly after gel formation before the gel was dried.

Results in FY2010 showed that full density was achieved in Sodalite 5A at $950{ }^{\circ} \mathrm{C}$. By adding the NBS-1 glass sintering aid, we showed that similar density could be achieved at $850^{\circ} \mathrm{C}$. However, the small crystallite grain sizes resulted in PCT responses that were higher than expected for the waste form. In 
general, in terms of phase assemblages, we saw that as firing temperature was increased, the concentrations of unreacted salts and zeolite phases were reduced. In some cases, sodalite increased as the unreacted salt and zeolite disappeared; however in most cases, this resulted in the formation of sodium aluminosilicates, e.g., nepheline and carnegieite. The sodium aluminosilicates are higher temperature phases than zeolites or sodalites, and thus both of these phases can convert to sodium aluminosilicates with increased heat-treatment temperature.

In FY2012, three different solution-based approaches were employed to make the sodalite waste forms with the same simulated $X \mathrm{Xl}_{20}$ salt. Several aspects were varied during the process including: the reactants, the amount of sintering aid added, the step in the process when the sintering aid was added, calcination, and the timing of the calcination step in the process. The three sodalite methods (SM-\#) used in the current study are $S M-1, S M-2$, and $S M-3$, simplified in Equations (2)-(4), above.

Equations (2)-(4) are listed with the $X \mathrm{Cl}_{20}$ denoted as " $\mathrm{NaCl}$ " but, in fact, the actual salt compositions contain $\mathrm{R}^{1+}, \mathrm{R}^{2+}$, and $\mathrm{R}^{3+}$ cations so the $\mathrm{R}: \mathrm{Cl}$ ratio is not $1: 1$ (by mole). The reactants were added in the proper amounts to obtain the targeted value for $\mathrm{Al}: \mathrm{Cl}(3: 1)$ and $\mathrm{Si}: \mathrm{Cl}(3: 1)$ on a molar basis where the alkali fraction $\left(\mathrm{R}^{1+}\right)$ was intentionally below the target of $4: 1\left(\mathrm{R}^{1+}: \mathrm{Cl}\right)$ to help influence the incorporation of alkaline earth and $\mathrm{Ln} X \mathrm{Cl}_{20}$ components into the sodalite structure. In our previous work we speculated that the alkaline earth and/or lanthanide ( $\mathrm{Ln}$ ) fraction could theoretically be incorporated into the sodalite structure but subsequently we have not observed this to be the case (Riley et al., 2012a).

\subsection{Tellurite Glasses: Background}

Tellurite glass was selected as the glass candidate host family for the $\mathrm{XCl}_{20}$ waste components in FY2009 because these glasses showed promise for moderate-to-high mass $\%$ loadings of up to $\sim 15 \mathrm{mass}_{0} \mathrm{XCl} \mathrm{l}_{20}$ and had very high volumetric waste loading — or storage density, $\rho_{\mathrm{sd}}$ - due to the high density of normal tellurite glass, $\rho_{\mathrm{sd}}>5 \times 10^{3} \mathrm{~kg} / \mathrm{m}^{3}$ (Crum et al., 2009).

The tellurite glass compositions that we fabricated in FY2009 covered a broad compositional space, were not optimized, and did not have high chemical durability. Thus, to better evaluate these glasses as a potential waste form, a literature study was conducted in FY2010 to identify promising candidate formulations for further investigation (Riley et al., 2010). This was followed up with a scoping study in which glasses were fabricated on a small scale $(\sim 5 \mathrm{~g})$ to evaluate the glass formation ability with and without the $X \mathrm{Cl}_{20}$ simulant. The scoping study was designed to assess some basic properties of a wide variety of simple tellurite glass compositions; these glasses were designated "S-series" glasses.

From the scoping studies, five systems were identified for further investigation and included tellurite glasses with the following additives: $\mathrm{PbO}, \mathrm{Al}_{2} \mathrm{O}_{3}+\mathrm{B}_{2} \mathrm{O}_{3}, \mathrm{WO}_{3}, \mathrm{P}_{2} \mathrm{O}_{5}$, and $\mathrm{ZnO}$. The successful formulations with these five systems were then made in 20-g batches and further characterized; these 20-g batches were designated "P-series" glasses. The P-series glasses were then characterized with assessments of a few of the properties including the following: volatility and pour viscosity during the melting process, density, microstructure, and chemical durability (assessed with the PCT). A summary of the characterization results from these 20-g glasses is presented in Table 2.6. As seen in Table 2.6, some of the properties for the P-series glasses varied widely, especially the volatility and the chemical durability. The lead-tellurite glasses performed the best and were further investigated in FY2011 and FY2012. The primary goals with this expanded study were to look at the glass structure and waste loading limits for the lead-tellurite system. 
Table 2.6. Summary results for the P-series tellurite glasses that were analyzed with the PCT. The "Glass" column lists those glasses batched on a 20-g scale for the PCT. Batch composition of each glass is listed in mass\% additive; the balance is $\mathrm{TeO}_{2}$. The composition of $\mathrm{XCl}_{20}$ is found in Table 2.1. The melting temperature is denoted $T_{\mathrm{M}}$. The "volatility" is ranked based on degree of "fuming" observed during the melt process, from none (0) to a lot (4). Viscosity $(\eta)$ was estimated as compared to standards. The overall deviation associated with the density measurements is $\pm 0.015 \times 10^{-3} \mathrm{~kg} / \mathrm{m}^{3}$ (Riley et al., 2010). Phase separation was determined based on visual ("Bulk") and microscopy ("Small") observations. The normalized sodium release during the PCT is given as $\left(N L_{\mathrm{Na}}\right)$.

\begin{tabular}{|c|c|c|c|c|c|c|c|c|}
\hline Glass & Batch Composition & $T_{\mathrm{M}}$ & Volatility & $\eta$ & $\rho_{\mathrm{b}} \times 10^{-3}$ & Phase & aration & $N L_{\mathrm{Na}}$ \\
\hline ID & (mass\% additive) & $\left({ }^{\circ} \mathrm{C}\right)$ & $(0-4)$ & $(\mathrm{cP})$ & & Bulk & Small & $\left(\mathrm{g} / \mathrm{m}^{2}\right)$ \\
\hline P-8 & $22.0 \% \mathrm{PbO}$ & 705 & 0 & $50-100$ & 6.12 & - & $\checkmark$ & - \\
\hline P-11 & $\begin{array}{l}19.8 \% \mathrm{PbO}, \\
10.0 \% \mathrm{XCl}_{20}\end{array}$ & 705 & 1 & 10 & 5.16 & - & - & 0.478 \\
\hline P-7 & $8.8 \% \mathrm{Al}_{2} \mathrm{O}_{3}, 9.0 \% \mathrm{~B}_{2} \mathrm{O}_{3}$ & 720 & 0 & 200 & 4.44 & - & $\checkmark$ & - \\
\hline $\mathrm{P}-2$ & $\begin{array}{l}7.9 \% \mathrm{Al}_{2} \mathrm{O}_{3}, 8.1 \% \mathrm{~B}_{2} \mathrm{O}_{3}, \\
10.0 \% \mathrm{Cl}_{20} \text {, }\end{array}$ & 690 & 3 & 50 & 4.13 & $\checkmark$ & $\checkmark$ & 6.71 \\
\hline P-9 & $25.0 \% \mathrm{WO}_{3}$ & 725 & 0 & 100 & 5.92 & $\checkmark$ & - & - \\
\hline $\mathrm{P}-12$ & $22.5 \% \mathrm{WO}_{3}, 10.0 X \mathrm{Cl}_{20}$ & 675 & 4 & 10 & 5.11 & $\checkmark$ & - & 10.3 \\
\hline $\mathrm{P}-5$ & $8.9 \% \mathrm{P}_{2} \mathrm{O}_{5}$ & 675 & 0 & 400 & 5.11 & - & - & - \\
\hline $\mathrm{P}-14$ & $8.1 \% \mathrm{P}_{2} \mathrm{O}_{5}, 10.0 \% X \mathrm{Cl}_{20}$ & 675 & 2 & $50-100$ & 4.72 & $\checkmark$ & $\checkmark$ & 86.0 \\
\hline $\mathrm{P}-4$ & $18.0 \% \mathrm{ZnO}$ & 730 & 0 & 100 & 5.49 & - & - & - \\
\hline $\mathrm{P}-13$ & $16.2 \% \mathrm{ZnO}, 10.0 \% \mathrm{XCl}_{20}$ & 730 & 1 & 10 & 4.67 & - & - & 88.9 \\
\hline
\end{tabular}

*The composition of the Echem salts was batched with a different $X \mathrm{Cl}_{20}\left(X \mathrm{Cl}_{y}\right)$ in glass P-2 (Riley et al., 2010). 


\section{METHODS}

\subsection{High-Halide Minerals}

\subsubsection{Solution-Based Fabrication Method for Halide Minerals in FY2010-FY2011}

Unique identifiers were applied to the various sets of high-halide minerals that we studied in FY2011 and are presented in Table 3.1. A solution-based process was used to form the high-halide minerals, followed with cold pressing and firing to convert the dried gel into a monolithic waste form. Figure 3.1 shows a schematic of the experimental apparatus; these are the steps taken during the process:

1) Simulated spent salt $\left(X_{C_{20}}\right.$, see Table 2.1) was dissolved in deionized water (DIW) - see Figure 3.1-(A).

2) Dissolved salt was heated to $\sim 65 \pm 5^{\circ} \mathrm{C}$ in the reaction beaker, which was heated by an oil bath (Figure 3.1). The solution was continuously stirred with a serpentine Pt wire throughout the formation process.

3) The silica $\left(\mathrm{SiO}_{2}\right)$ or germania $\left(\mathrm{GeO}_{2}\right)$ source was slowly added as a separate solution to the dissolved salt solution-see Figure 3.1-(B). The Si-O or Ge-O source is designated in Table 2.4 for each sample series.

4) The solution was then gelled by slow addition of sodium aluminate solution-see Figure 3.1-(C).

5) For some of the batches, a sintering aid (1-7 mass\%) was added at this stage in the form of powder (not shown in Figure 3.1). See Table 3.2 for a list of the sintering aids studied along with the glass compositions and a few properties.

6) The gel was stirred and heated for 10-30 min to drive off excess water.

7) Lastly, the gel-containing beaker was heated at $105^{\circ} \mathrm{C}$ overnight to complete the drying process.

The dried material was a moderately bound fine powder. The amounts of waste simulant, colloidal silica, and sodium aluminate added were determined by the target waste form composition.

Table 3.1. Labels for the various sets of high-halide minerals fabricated in FY2011. Here, CS, TEOS, and Ge-EtO denote that samples were made with colloidal silica, tetraethyl orthosilicate, and germanium(IV) ethoxide, respectively. See Table 2.4 for the S5A and S6A compositions.

\begin{tabular}{ll}
\hline Sample series & Description \\
\hline S5A-CS & $\begin{array}{l}\text { Samples were made with the 5A composition and CS as the 4-coordinated Group IV } \\
\text { component } \\
\text { S5C-TEOS }\end{array}$ \\
Samples were made with the 5A composition and TEOS as the 4-coordinated Group \\
IV component \\
S5D-GeEtO & $\begin{array}{l}\text { Samples were made with the 5A composition and Ge-EtO as the 4-coordinated Group } \\
\text { IV component }\end{array}$ \\
S6A-CS & $\begin{array}{l}\text { Samples were made with the 5A composition and CS as the 4-coordianted Group IV } \\
\text { component but the NBS-1 glass binder was added }\end{array}$ \\
\end{tabular}




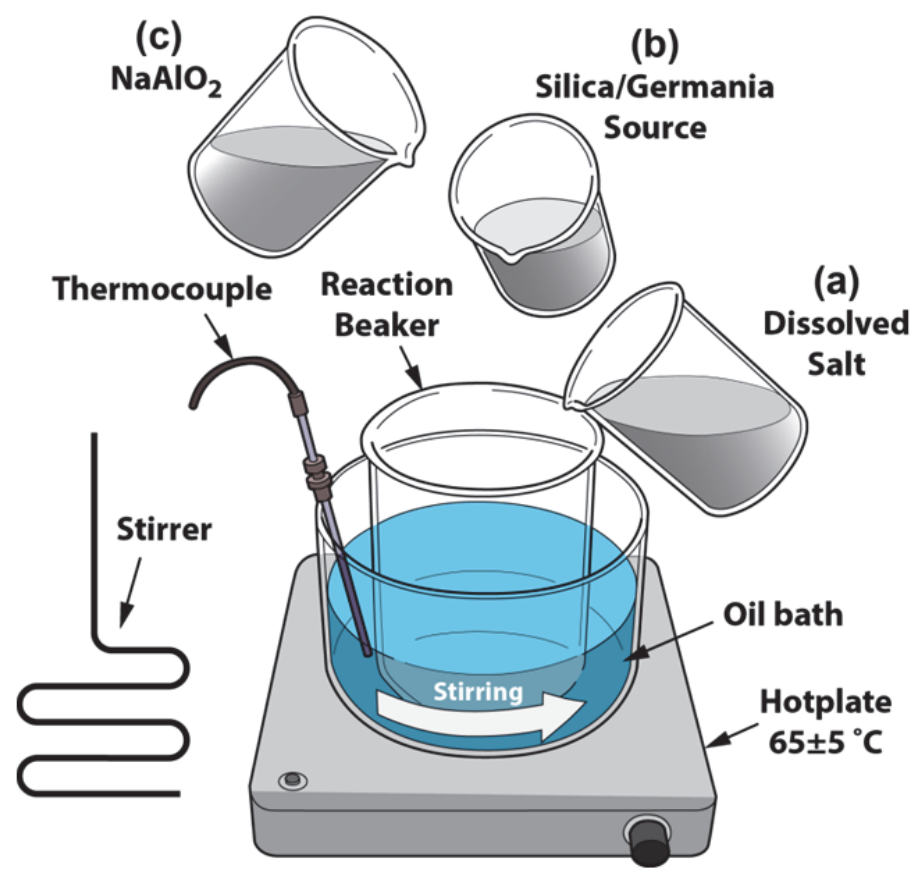

Figure 3.1. Schematic of the simplified solution-based process.

For the silica-based sodalites that were made with TEOS as the silica source, a representative process included the following:

1) Added $30.0 \mathrm{~mL}$ of salt solution (see Table 2.5) to a $250 \mathrm{~mL}$ beaker with a platinum stirring rod

2) Added $52.8 \mathrm{~mL}$ of TEOS to the beaker ( $99.0 \%$ purity, Sigma-Aldrich, St. Louis, MO)

3) Added $51.0 \mathrm{~mL}$ of $\mathrm{NaAlO}_{2}$ (38\% solution, USALCO, LLC, Baltimore, MD)

4) Added $13.8 \mathrm{~mL}$ of absolute ethanol

5) Added $2.05 \mathrm{~g}$ of glass (on a 5 mass $\%$ basis)

6) After the glass was added, the viscosity greatly increased to form a solid in an exothermic reaction ( $\sim 30$ seconds)

7) Beaker was placed in a special drying oven overnight.

8) The resulting product was powdered and pressed into pellets for analysis.

The glass sintering aids that we studied in FY2011 are presented in Table 3.2. The sodium borosilicate glass sintering aids, denoted as SA-\#\#\#, were selected based on their lower melting temperatures and were labeled based on the predicted temperatures $\left(\sim 700,750,800\right.$, and $\left.850^{\circ} \mathrm{C}\right)$ at which the viscosity was predicted to be $100 \mathrm{~Pa} \cdot \mathrm{s}$ with SciGlass models (v 7.0, ITC, Inc., Newton, MA). The NaAlP-B4 is discussed in detail elsewhere (Donald et al., 2007) and P-8 is a tellurite glass discussed here as well as in our previous work (Riley et al., 2010). The NaAlP-B4 glass was selected based on past success as a sintering aid for an apatite mineral waste form (Donald et al., 2007). The P-8 glass is one of the tellurite glasses discussed here that was selected because it has a high affinity for the Echem salts and a good chemical durability (Riley et al., 2010). Each of these binders was fabricated with a melt-quench technique and then powdered for addition in Step 5, above. 
Table 3.2. Compositions (in mass\%) of glass sintering aid (SA) additions tested in FY2011. The NBS-1 (Vienna et al., 1998) glass from FY2010 studies is listed for comparison purposes (Riley et al., 2010). Also, some properties are listed and include the temperature at which the viscosity is $100 \mathrm{~Pa} \cdot \mathrm{s}$, the melting temperature $\left(T_{\mathrm{M}}\right)$, and the bulk density measured with a helium pycnometer. Some of the data was not available for $\mathrm{TePbO}\left(\mathrm{TPb}_{0}\right)$ or NaAlP-B4 for comparison, e.g., $T_{\mathrm{L}}$ represents the liquidus temperature, in place of the $T_{\mathrm{M}}$ for NaAlP-B4.

\begin{tabular}{lccccccc}
\hline Components & NBS-1 & SA-700 & SA-750 & SA-800 & SA-850 & NaAlP-B4 & TePbO \\
\hline $\mathrm{SiO}_{2}$ & 60.00 & 28.57 & 40.83 & 42.5 & 48.31 & - & - \\
$\mathrm{Na}_{2} \mathrm{O}$ & 15.00 & 38.32 & 37.49 & 32.88 & 34.89 & 40.0 & - \\
$\mathrm{B}_{2} \mathrm{O}_{3}$ & 10.00 & 33.11 & 21.68 & 24.62 & 16.80 & 2.0 & - \\
$\mathrm{Li}_{2} \mathrm{O}$ & 5.00 & - & - & - & - & - & - \\
$\mathrm{Al}_{2} \mathrm{O}_{3}$ & 5.00 & - & - & - & - & 19.0 & - \\
$\mathrm{ZrO}_{2}$ & 5.00 & - & - & - & - & - & - \\
$\mathrm{P}_{2} \mathrm{O}_{5}$ & - & - & - & - & - & 39.0 & - \\
$\mathrm{TeO}_{2}$ & - & - & - & - & - & - & 78.00 \\
$\mathrm{PbO}_{T} @ 100 \mathrm{~Pa} \cdot \mathrm{s}$ & 963 & 700 & 751 & 805 & 850 & (b) & $<600^{\text {(a) }}$ \\
$T_{\mathrm{M}},{ }^{\circ} \mathrm{C}$ & $1250^{(\mathrm{a})}$ & 850 & 900 & 950 & 1000 & $742\left(T_{\mathrm{L}}\right)$ & $650^{(\mathrm{a})}$ \\
$\rho_{\mathrm{b}}, \times 10^{3} \mathrm{~kg} / \mathrm{m}^{3}$ & 2.561 & 2.527 & 2.542 & 2.551 & 2.544 & (b) & 6.169 \\
\hline
\end{tabular}

(a) These values are estimated

(b) These values are unknown

\subsubsection{Solution-Based Fabrication Method for Halide Minerals in FY2012}

This following section outlines the approach for making the SM-\# sodalites in FY2012. Some of the precursor solutions were heated to assist dissolution of the solute in the solvents. However, the individual precursor solutions were not actively heated during the mixing process. Several different processing combinations were studied and included the following:

(1) The sample was made and dried

(2) The sample was dried and calcined at $600{ }^{\circ} \mathrm{C}$

(3) The sample was dried, calcined at $600{ }^{\circ} \mathrm{C}$, a sintering aid was added dry, and mixed

(4) A sintering aid was added to the solution-based process as a final step, mixed in, and the sample was dried

A sintering aid (SA-800, Table 3.2) was added to the solution-based process as a final step, mixed in, the sample was dried, and then calcined at $600^{\circ} \mathrm{C}$. 
Table 3.3. Sample name designations for solution-derived sodalites in FY12.

\begin{tabular}{ll}
\hline Designation & Experimental Details \\
\hline SM\#-AM & As made and dried (no binder added during solution process) \\
SM\#-AM-C & SM\#-AM is dried and calcined at $600{ }^{\circ} \mathrm{C}$ for $3 \mathrm{~h}$ in air \\
\hline SM\#-5\% & $\begin{array}{l}\text { SM\#-AM is prepared with } 5 \% \text { sintering aid added during solution process and } \\
\text { dried }\end{array}$ \\
SM\#-AM-5\% & $\begin{array}{l}5 \% \text { sintering aid is added to dried SM\#-AM } \\
\text { SM\#-5\%-C }\end{array}$ \\
SM\#-C-5\% & $5 \%$ is calcined at $600{ }^{\circ} \mathrm{C}$ for 3 hours in air and milled \\
SM\#-10\% & $\begin{array}{l}\text { SM\#-AM is prepared with } 10 \% \text { sintering aid added during solution process and } \\
\text { dried }\end{array}$ \\
SM\#-AM-10\% & $10 \%$ sintering aid is added to dried SM\#-AM \\
SM\#-10\%-C & SM\#-10\% is calcined at $600{ }^{\circ}$ C for 3 hours in air and milled \\
SM\#-C-10\% & $10 \%$ sintering aid is added to dried SM\#-AM-C \\
\hline SM\#-VS & SM\#-5\%-C samples that were pressed at different pressures
\end{tabular}

*All samples were milled prior to pellets being pressed. In each case except SM\#-VS, these samples were pressed at $3.5 \times 10^{8} \mathrm{~Pa}\left(5.0 \times 10^{4} \mathrm{PSI}\right)$. All \%'s are by mass.

\subsubsection{Gibbsite Process (SM-1, Equation (2))}

For this technique, $21.538 \mathrm{~g}$ of gibbsite powder, $\mathrm{Al}(\mathrm{OH})_{3}(<8 \mu \mathrm{m}$, NOAH Technologies Corporation, San Antonio, TX), was mixed in a $150 \mathrm{~mL}$ beaker containing $50 \mathrm{~mL}$ of $5.52 \mathrm{M} \mathrm{NaOH}$ (Fisher Scientific, Pittsburgh, PA) at $70 \pm 5{ }^{\circ} \mathrm{C}$ for $1 \mathrm{~h}$. Heating was applied to assist in $\mathrm{Al}(\mathrm{OH})_{3}$ dissolution but the sample was not completely dissolved after this duration as was expected based on $\mathrm{pH}$-temperature solubility limitations according to the literature (Li et al., 2005; Misra, 1970). In a separate $250 \mathrm{~mL}$ beaker, $30 \mathrm{~mL}$ of the $X_{\mathrm{Cl}_{20}}$ salt solution (Table 2.5) was added along with $39.72 \mathrm{~mL}$ of LUDOX ${ }^{\circledR} \mathrm{HS}-40$ colloidal silica (40 mass $\%$ solids, Sigma-Aldrich, St. Louis, MO) solution and stirred for 5 min. Next, the $\mathrm{Al}(\mathrm{OH})_{3^{-}}$ $\mathrm{NaOH}$ solution was added to the salt-colloidal silica solution and mixed for $1 \mathrm{~h}$ without active heating. In some samples, a sintering aid was added during the last 5 minutes of mixing. The sample was then dried in an oven at $105^{\circ} \mathrm{C}$ overnight. Once dry, the sample was ground in a tungsten carbide mill for 4 minutes to create a homogeneous product.

\subsubsection{Sodium Aluminate Process (SM-2, Equation (3))}

For this technique, $22.598 \mathrm{~g}$ of sodium aluminate powder, $\mathrm{NaAlO}_{2}$ (Riedel-de Haën, Germany), was added to $75 \mathrm{~mL}$ of deionized water in a $150 \mathrm{~mL}$ beaker and stirred overnight at $70 \pm 5^{\circ} \mathrm{C}$ covered with a watch glass to minimized evaporation of the water. In a separate $250 \mathrm{~mL}$ beaker, $30 \mathrm{~mL}$ of the $X \mathrm{Cl}_{20}$ salt solution (Table 2.5) was added and $39.72 \mathrm{~mL}$ of LUDOX HS-40 colloidal silica solution was added to the $X \mathrm{Cl}_{20}$ salt solution and stirred for $5 \mathrm{~min}$. Next, the $\mathrm{NaAlO}_{2}$ solution was added to the $X \mathrm{Cl}-\mathrm{SiO}_{2}$ solution and mixed for 1 hour. In some samples, a sintering aid was added during the last 5 minutes of mixing. The sample was then dried overnight at $105^{\circ} \mathrm{C}$ in an oven. Once dry, the sample was ground in a tungsten carbide mill for 4 minutes to create a homogeneous product.

\subsubsection{Metakaolin Process (SM-3, Equation (4))}

For this technique, kaolin clay (Sigma-Aldrich) was heat treated at $\sim 850{ }^{\circ} \mathrm{C}$ for $17 \mathrm{~h}$ in air to convert to metakaolin $\left(\mathrm{Al}_{2} \mathrm{Si}_{2} \mathrm{O}_{7}\right)$ through the dehydroxylation process as described in Equation 5 (Konan et al., 2009). The $\mathrm{Al}_{2} \mathrm{Si}_{2} \mathrm{O}_{7}$ was analyzed with $\mathrm{XRD}$ and found to be amorphous as is consistent with the literature (Konan et al., 2009). Once converted, $30.667 \mathrm{~g}$ of $\mathrm{Al}_{2} \mathrm{Si}_{2} \mathrm{O}_{7}$ was added to $50 \mathrm{~mL}$ of $5.52 \mathrm{M}$ $\mathrm{NaOH}$ while mixing and heating at $70 \pm 5^{\circ} \mathrm{C}$ for one hour. Finally, $30 \mathrm{~mL}$ of the $X \mathrm{Cl}_{20}$ salt solution (Table 2.5) was added and the solution was mixed for $1 \mathrm{~h}$. In some samples, a sintering aid was added during the 
last 5 minutes of mixing. The sample was then dried in an oven at $105^{\circ} \mathrm{C}$ overnight then ground for 4 minutes in a tungsten carbide mill.

$$
2 \mathrm{Al}_{2} \mathrm{Si}_{2} \mathrm{O}_{5}(\mathrm{OH})_{4} \stackrel{850^{\circ} \mathrm{C}}{\longrightarrow} 2 \mathrm{Al}_{2} \mathrm{Si}_{2} \mathrm{O}_{7}+4 \mathrm{H}_{2} \mathrm{O}
$$

\subsubsection{Measuring Solution $\mathrm{pH}$}

For each of these processes, the $\mathrm{pH}$ was monitored with a probe and digital readout that were calibrated with standards. Equilibration times were $\sim 1-5 \mathrm{~min}$. As a check, $\mathrm{pH}$ paper was also used to confirm.

\subsubsection{Pellet Preparation}

Once the samples were ground to a powder, in some cases, the sintering aid was added (dry) at this stage and the samples were homogenized again in the mill. Then, specimens were weighed, pressed into pellets with a combination of techniques. In order to keep the starting pellet dimensions similar and aid in postprocessing comparisons, in all cases a total mass of $\sim 2.5 \mathrm{~g}$ of powder were added (including sintering aid if added dry). The raw or mixed powders were loaded into a cylindrical die, placed in a uniaxial press (UAP) at room temperature, and pressed to the minimum pressure required to form and maintain a pellet shape of $\sim 21.4 \mathrm{~mm}$ diameter and $\sim 4-5 \mathrm{~mm}$ thick, i.e., $2.5 \times 10^{7} \mathrm{~Pa}\left(3.6 \times 10^{3}\right.$ pounds per square inch or PSI). Higher applied pressures at this stage were attempted in our previous work and led to distortions in pellet dimensions after firing (Riley et al., 2010). Pellets were then isostatically pressed (ISP) at room temperature at $3.4 \times 10^{8} \mathrm{~Pa}\left(5.0 \times 10^{4} \mathrm{PSI}\right)$.

\subsubsection{Firing Pellets}

The pellets were then placed on a dense alumina sintering plate and loaded into a Lindberg furnace (Thermal Product Solutions, Rochester, NY). Specimens were fired by ramp heating at $1{ }^{\circ} \mathrm{C} \min ^{-1}$ up to the maximum firing temperature of interest (i.e., $650,750,850$, or $950^{\circ} \mathrm{C}$ ), held for $8 \mathrm{~h}$, and then furnace cooled at $\sim 10{ }^{\circ} \mathrm{C} \mathrm{min}{ }^{-1}$. Some specimens were calcined at $600{ }^{\circ} \mathrm{C}$ for 3 hours, at different steps in the process before firing, in order to evaluate the effect of calcination on bulk density of the waste form.

\subsubsection{Cold-Pressing Pressure Variation Study (PVS) and Evaluation of Method Accuracy}

After the results from the SM-\# pellets were analyzed, a set of conditions was selected for further tests to assess the accuracy of the sodalite and density measurements as well as the effect of pressing pressure on the properties of the pellets fired at different temperatures. Each selected specimen from the original batch for this sub-study was run in triplicate to assess the variability in the sodalite and density measurements. The specimens selected for this study were those from SM-1, SM-2, and SM-3 where 5\% of SA800 sintering aid was added at the end of the solution-based process, the samples were then dried, calcined, and fired; these specimens were SM1-5\%-C- $850^{\circ} \mathrm{C}, \mathrm{SM} 2-5 \%-\mathrm{C}-850^{\circ} \mathrm{C}$, and $\mathrm{SM} 3-5 \%-\mathrm{C}-750^{\circ} \mathrm{C}$, respectively. Four different pressures were used for making the pellets and included (1) $1.2 \times 10^{7} \mathrm{~Pa}$ UAP $\left(1.8 \times 10^{3} \mathrm{PSI}\right)$, (2) $2.5 \times 10^{7} \mathrm{~Pa}$ with UAP, (3) $2.5 \times 10^{7} \mathrm{~Pa}$ with UAP $+1.7 \times 10^{8} \mathrm{~Pa}$ with $\mathrm{ISP}\left(2.5 \times 10^{4} \mathrm{PSI}\right)$, and (4) $2.5 \times 10^{7} \mathrm{~Pa}$ with UAP $+3.4 \times 10^{8} \mathrm{~Pa}$ with ISP.

\subsubsection{Spark Plasma Sintering}

Specimens of SM1-5\%-C-850 ${ }^{\circ}$, SM2-5\%-C- $850^{\circ} \mathrm{C}$, and SM3-5\%-C-750 ${ }^{\circ} \mathrm{C}$ were pressed at $2.5 \times 10^{7} \mathrm{~Pa}$ with UAP and then hot-pressed with spark plasma sintering at California Nanotechnologies (Cerritos, CA) using a SPS-515S (DR.SINTER LAB, Fuji Electronic Industrial Co., Japan). Specimens were heated in graphite dies at a heating rate of $\sim 50-60^{\circ} \mathrm{C} / \mathrm{min}$ with a hold at $200^{\circ} \mathrm{C}$ for degasing and held there until the vacuum level equilibrated. Then, the specimens were heated manually until the displacement of the die push rod either plateaued or started to drop with increased heating. This occurred at $\sim 554, \sim 526$, and $\sim 551{ }^{\circ} \mathrm{C}$ for the three specimens, respectively, at which point they were subjected to $\sim 52 \mathrm{MPa}$. Each sample was heated at these temperatures for $\sim 10 \mathrm{~min}$ and then cooled to room temperature. 


\subsection{Tellurite Glasses}

All of the tellurite glasses discussed here were batched from oxides, boric acid, and chlorides of high purity (all $>99 \%$ pure). The $X \mathrm{Cl}_{20}$ components that were added to the waste-loaded glasses were added in the ratios presented in Table 3.4. In all cases, the raw materials were mixed in a mortar and pestle, the powders were then placed in a $\mathrm{Pt} / 10 \% \mathrm{Rh}$ crucible with a $\mathrm{Pt} / 10 \% \mathrm{Rh}$ lid, melted for 30 minutes, and quenched by pouring on a stainless steel plate. The as-quenched glasses were subsequently prepared for various tests. The variables in the fabrication parameters included the batch mass and the melting temperature $\left(T_{\mathrm{M}}\right)$. The glasses discussed below are grouped into different sections based on the purpose of study and include the following: 1) compositional variation study glasses, 2) lead-tellurite variation study glasses, and 3) structural investigation study glasses. The specific values used for the compositional variation study glasses are presented in Table 2.6 and are listed separately in the text for each set of glasses.

Table 3.4. Composition of the $X \mathrm{Cl}_{20}$ salt added to the tellurite glasses along with the melting temperatures for some of the components (in order of mass fraction from largest to smallest). The melting temperature $\left(T_{\mathrm{M}}\right)$ and bulk density $\left(\rho_{\mathrm{b}}\right)$ are also included for each (Haynes, 2011).

\begin{tabular}{lcccc}
\hline Component & Mass\% & Mole $\%$ & $T_{\mathrm{M}},{ }^{\circ} \mathrm{C}$ & $\rho_{\mathrm{b}}, \times 10^{-3} \mathrm{~kg} / \mathrm{m}^{3}$ \\
\hline $\mathrm{KCl}$ & 37.99 & 33.83 & 771 & 1.99 \\
$\mathrm{LiCl}$ & 29.89 & 46.82 & 610 & 2.07 \\
$\mathrm{NaCl}$ & 11.71 & 13.30 & 801 & 2.17 \\
$\mathrm{NdCl}_{3}$ & 5.36 & 1.42 & 759 & 4.13 \\
$\mathrm{CsCl}$ & 3.91 & 1.54 & 646 & 3.99 \\
$\mathrm{CeCl}_{3}$ & 3.51 & 0.95 & 807 & 3.97 \\
$\mathrm{LaCl}_{3}$ & 2.29 & 0.62 & 858 & 3.84 \\
$\mathrm{BaCl}_{2}$ & 2.01 & 0.64 & 961 & 3.90 \\
$\mathrm{PrCl}_{3}$ & 1.72 & 0.46 & 786 & 4.00 \\
$\mathrm{SmCl}_{3}$ & 1.61 & 0.42 & 682 & 4.46 \\
\hline
\end{tabular}

The naming convention of the glasses presented in the FY2010 report was changed in this report to make it easier to track the glass by composition as opposed to tracking the glasses by the sequential-numbering sample identification. However, both of these naming conventions are retained here for cross-referencing to our other work (see Table 3.5).

\subsubsection{Compositional Variation Study Glasses}

The glasses for the compositional variation study include ten "P-\# series" glasses that were fabricated in FY2010 and were previously discussed in our FY2010 report, but were renamed here for easier crossreference to the glass compositions (Table 3.5) (Riley et al., 2010). The structural characterizations of these glasses, i.e., XRD and Raman spectroscopy, will be discussed in section 4.3.1 in more detail. All of these glasses were fabricated on a $20 \mathrm{~g}$ scale and $T_{\mathrm{M}}$ values are listed in our FY2010 report. 
Table 3.5. Compositions (in mole\%) from tellurite glass compositional variation study batched with and without $X \mathrm{Cl}_{20}$ additions.* These values are also presented in mass\% in our earlier work (Riley et al., 2010), although they are presented here as mole $\%$ because that is more appropriate for the discussion of the glass structure. Each of the " $+\mathrm{Cl}$ " glasses were batched with a target of 10 mass $\% \mathrm{XCl}_{20}$-loading.

\begin{tabular}{|c|c|c|c|c|c|c|c|c|c|c|}
\hline $\begin{array}{l}\text { Composition } \\
\text { Glass ID }\end{array}$ & $\begin{array}{l}\mathrm{Pb} \\
\mathrm{P}-8\end{array}$ & $\begin{array}{c}\mathrm{Pb}+\mathrm{Cl} \\
\mathrm{P}-11 \\
\end{array}$ & $\begin{array}{c}\mathrm{A} 1 / \mathrm{B} \\
\mathrm{P}-7\end{array}$ & $\begin{array}{c}\mathrm{Al} / \mathrm{B}+\mathrm{Cl} \\
\mathrm{P}-2 \\
\end{array}$ & $\begin{array}{c}\text { W } \\
\text { P-9 }\end{array}$ & $\begin{array}{l}\mathrm{W}+\mathrm{Cl} \\
\mathrm{P}-12 \\
\end{array}$ & $\begin{array}{c}\mathrm{P} \\
\mathrm{P}-5\end{array}$ & $\begin{array}{l}\mathrm{P}+\mathrm{Cl} \\
\mathrm{P}-14 \\
\end{array}$ & $\begin{array}{l}\mathrm{Zn} \\
\mathrm{P}-4\end{array}$ & $\begin{array}{c}\mathrm{Zn}+\mathrm{Cl} \\
\mathrm{P}-13 \\
\end{array}$ \\
\hline $\mathrm{TeO}_{2}$ & 83.2 & 64.8 & 70.5 & 59.4 & 81.3 & 63.1 & 90.0 & 71.2 & 69.9 & 56.9 \\
\hline $\mathrm{PbO}$ & 16.8 & 13.1 & - & - & - & - & - & - & - & - \\
\hline $\mathrm{Al}_{2} \mathrm{O}_{3}$ & - & - & 11.8 & 10.0 & - & - & - & - & - & - \\
\hline $\mathrm{B}_{2} \mathrm{O}_{3}$ & - & - & 17.7 & 14.9 & - & - & - & - & - & - \\
\hline $\mathrm{WO}_{3}$ & - & - & - & - & 18.7 & 14.5 & - & - & - & - \\
\hline $\mathrm{P}_{2} \mathrm{O}_{5}$ & - & - & - & - & - & - & 10.0 & 7.9 & - & - \\
\hline $\mathrm{ZnO}$ & - & - & - & - & - & - & - & - & 30.1 & 24.5 \\
\hline $\mathrm{BaCl}_{2}$ & - & 0.142 & - & 0.192 & - & 0.144 & - & 0.134 & - & 0.119 \\
\hline $\mathrm{CeCl}_{3}$ & - & 0.210 & - & 0.245 & - & 0.212 & - & 0.198 & - & 0.175 \\
\hline $\mathrm{CsCl}$ & - & 0.342 & - & 0.611 & - & 0.346 & - & 0.322 & - & 0.286 \\
\hline $\mathrm{KCl}$ & - & 7.50 & - & 8.06 & - & 7.60 & - & 7.07 & - & 6.27 \\
\hline $\mathrm{LaCl}_{3}$ & - & 0.138 & - & 0.160 & - & 0.139 & - & 0.130 & - & 0.115 \\
\hline $\mathrm{LiCl}$ & - & 10.4 & - & 3.48 & - & 10.5 & - & 9.78 & - & 8.68 \\
\hline $\mathrm{NaCl}$ & - & 2.95 & - & 2.38 & - & 2.99 & - & 2.78 & - & 2.47 \\
\hline $\mathrm{NdCl}_{3}$ & - & 0.315 & - & 0.372 & - & 0.319 & - & 0.297 & - & 0.263 \\
\hline $\mathrm{PrCl}_{3}$ & - & 0.102 & - & 0.119 & - & 0.104 & - & 0.0963 & - & 0.0855 \\
\hline $\mathrm{SmCl}_{3}$ & - & 0.0925 & - & 0.111 & - & 0.0936 & - & 0.0871 & - & 0.0773 \\
\hline
\end{tabular}

*A different $X \mathrm{Cl}_{20}$ composition was used for the $\mathrm{Al} / \mathrm{B}+\mathrm{Cl}$ glass than for the other $X \mathrm{Cl}_{20}$-containing glasses (Riley et al., 2010).

\subsubsection{Lead-Tellurite Waste-Loading Variation Study Glasses}

The second set of tellurite glasses fabricated and studied in FY2011 was a series of lead-tellurite glasses with a fixed target $\mathrm{TeO}_{2}: \mathrm{PbO}$ ratio (by mass) of 3.5455 and a fixed target ratio of $\mathrm{Te}: \mathrm{Pb}$ (by mole) of 4.9583, both based on the P-8 and P-11 glasses. This glass system was chosen based on the chemical durability of these glasses according to the FY2010 results. The fraction of $X^{2} l_{20}$ added to the baseline lead-tellurite glass $\left(\mathrm{TPb}_{0}\right)$ was varied from 0 to 22.5 mass $\%$. The compositions of these glasses are listed in Table 3.6, in which the ratios between the $\mathrm{XCl}_{20}$ components can be extrapolated from Table 3.5. All of these glasses were fabricated in 30 -g batches and melted at $700{ }^{\circ} \mathrm{C}$ for 30 minutes. It is worth noting that the $\mathrm{TPb}_{14}$ glass was batched two times to verify the properties due to some anomalies in the data for this glass. 
Table 3.6. Compositions of lead-tellurite variation study glasses in both mass $\%$ and mole $\%$. The subscripts on the glass identification (ID) represent the $X \mathrm{Cl}_{20}$-loading in mass $\%$. $\mathrm{TPb}_{0}$ and $\mathrm{TPb}_{10}$ represent compositions similar to P-8 and P-11, respectively, from previous work (Riley et al., 2010) but these glasses were all melted at $700{ }^{\circ} \mathrm{C}$ and with a different target mass ( $30 \mathrm{~g}$ vs. $20 \mathrm{~g}$ ). The sequential glass numbering identification, or "Alternate ID", is also provided for tracking purposes.

\begin{tabular}{ll|ccc|ccc}
\hline \multirow{2}{*}{ Glass ID } & \multirow{2}{*}{ Alternate ID } & \multicolumn{3}{|c|}{ Mass\% } & \multicolumn{3}{c}{ Mole\% } \\
\cline { 3 - 7 } & & $\mathrm{TeO}_{2}$ & $\mathrm{PbO}$ & $X \mathrm{Cl}_{20}$ & $\mathrm{TeO}_{2}$ & $\mathrm{PbO}$ & $X \mathrm{Cl}_{20}$ \\
\hline $\mathrm{TPb}_{0}$ & $\mathrm{~T} 2 \mathrm{G}-21$ & 78.0 & 22.0 & 0.0 & 83.2 & 16.8 & 0.0 \\
$\mathrm{TPb}_{5}$ & $\mathrm{~T} 2 \mathrm{G}-22$ & 74.1 & 20.9 & 5.0 & 73.3 & 14.8 & 11.9 \\
$\mathrm{TPb}_{10}$ & $\mathrm{~T} 2 \mathrm{G}-23$ & 70.2 & 19.8 & 10.0 & 64.8 & 13.1 & 22.1 \\
$\mathrm{TPb}_{12.5}$ & $\mathrm{~T} 2 \mathrm{G}-24$ & 68.3 & 19.2 & 12.5 & 60.9 & 12.3 & 26.8 \\
$\mathrm{TPb}_{14}$ & $\mathrm{~T} 2 \mathrm{G}-31$ & 67.1 & 18.9 & 14.0 & 58.7 & 11.8 & 29.5 \\
$\mathrm{TPb}_{15}$ & $\mathrm{~T} 2 \mathrm{G}-26$ & 66.3 & 18.7 & 15.0 & 57.3 & 11.5 & 31.2 \\
$\mathrm{TPb}_{17.5}$ & $\mathrm{~T} 2 \mathrm{G}-25$ & 64.4 & 18.1 & 17.5 & 53.9 & 10.9 & 35.2 \\
$\mathrm{TPb}_{22.5}$ & $\mathrm{~T} 2 \mathrm{G}-30$ & 60.5 & 17.0 & 22.5 & 47.7 & 9.6 & 42.7 \\
\hline
\end{tabular}

\subsubsection{Structural Variation Study Glasses}

The last series of tellurite glasses, the structural variation study glasses, was designed to provide better insight into the effects of composition on the glass structure. Several different sets of glasses were fabricated in which various components were altered individually, in most cases. These different sets of glasses are presented and discussed below.

$\underline{\text { Set 1: } \mathrm{TeO}_{2}}: \underline{\mathrm{PbO}}$. The purpose of these glasses was to investigate the glass formation in lead-tellurite glasses with a lower mass fraction of $\mathrm{PbO}$ than that used for the lead-tellurite variation study glasses, i.e., 22 mass $\% \mathrm{PbO}$ (with a $\mathrm{TeO}_{2}$ balance) where $\mathrm{TeO}_{2}: \mathrm{PbO}=3.545$ (by mass). The compositions of this set of glasses are presented in Table 3.7.

Table 3.7. Compositions for T2G-21, T2G-21b, T2G-21c, and T2G-21d (in mole\%) in which the mass $\%$ of $\mathrm{PbO}$ was 22, 20, 18, and 16 mass \%, respectively. The baseline glass for this series is $\mathrm{T} 2 \mathrm{G}-21$, listed in bold type.

\begin{tabular}{lcccc}
\hline Glass ID: & T2G-21 & T2G-21b & T2G-21c & T2G-21d \\
\hline $\mathrm{TeO}_{2}$ & $\mathbf{8 3 . 2 2}$ & 84.83 & 86.43 & 88.01 \\
$\mathrm{PbO}$ & $\mathbf{1 6 . 7 8}$ & 15.17 & 13.57 & 11.99 \\
\hline$m\left(\mathrm{TeO}_{2}: \mathrm{PbO}\right)$ & $\mathbf{3 . 5 5}$ & 4.00 & 4.56 & 5.25 \\
\hline$M(\mathrm{~Pb}: \mathrm{Te})$ & $\mathbf{0 . 2 0 2}$ & 0.179 & 0.157 & 0.136 \\
\hline
\end{tabular}

Set 2: $\mathbf{P b O} / \mathbf{P b C l}_{2}$. The purpose of this study was to look at the structural effect of the anion in the leadtellurite glasses where lead was added as either $\mathrm{PbO}$ or $\mathrm{PbCl}_{2}$ and the Te: $\mathrm{Pb}$ molar ratio was fixed at 0.202 , to match that of T2G-21 $\left(\mathrm{TPb}_{0}\right)$, where "Pb" = total $\mathrm{Pb}$. This variation method was chosen so the amount of $\mathrm{Te}$ and $\mathrm{Pb}$ atoms interacting in the glass would be equivalent between the glasses. These compositions are presented in Table 3.8. 
Table 3.8. Composition of $\mathrm{PbCl}_{2}-\mathrm{PbO}$ compositional variation glasses to study the structural effect of the anion in the lead-tellurite glasses. For all of the glasses in this series, the molar ratio, $M$, of $\mathrm{Pb}$ :Te was fixed at 0.202 . The baseline glass, T2G-21, is listed in bold type for reference.

\begin{tabular}{lccc}
\hline Glass ID: & T2G-21 & T2G-32 & T2G-33 \\
\hline $\mathrm{TeO}_{2}$ & $\mathbf{8 3 . 2 2}$ & Mole\% \\
$\mathrm{PbO}$ & $\mathbf{1 6 . 7 8}$ & - & 83.22 \\
$\mathrm{PbCl}$ & - & 16.78 & 8.22 \\
\hline & \multicolumn{3}{c}{ Mass\% } \\
\hline $\mathrm{TeO}_{2}$ & $\mathbf{7 8 . 0 0}$ & 74.00 & 75.95 \\
$\mathrm{PbO}$ & $\mathbf{2 2 . 0 0}$ & - & 10.71 \\
$\mathrm{PbCl}$ & - & 26.00 & 13.34 \\
\hline$M(\mathrm{~Pb}: \mathrm{Te})$ & $\mathbf{0 . 2 0 2}$ & 0.202 & 0.202 \\
\hline
\end{tabular}

Set 3: $X \mathbf{O}_{20}$ versus $X \mathbf{X l}_{20}$. The purpose of this study was to look at the glass structural effect of the $X \mathrm{Cl}_{20}$ anions. Thus, for T2G-34, all of the $X \mathrm{Cl}_{20}$ components were replaced by the oxide versions where the cation ratios (by mole) were maintained from the 5 mass $\% X_{20}$-loaded glass, $\mathrm{T} 2 \mathrm{G}-22$, and the $\mathrm{XO}_{20}$-loaded glass. Thus, ratios of $\mathrm{Te}$ and $\mathrm{Pb}$ to each of the waste component cations, (Cat.), remained fixed between the two glasses, i.e., Te:Cat. and $\mathrm{Pb}$ :Cat., respectively. The ratio of $\mathrm{Te}$ and $\mathrm{Pb}$ to the total moles of the waste component cations were fixed at 6.16 and 1.24, respectively, for both T2G-22 and T2G-34. The compositions, corresponding ratios, and the $\mathrm{XO}_{20}$ composition are presented in Table 3.9.

Table 3.9. Summary of compositions for the waste-component-anion substitution glasses as well as the composition of $\mathrm{XO}_{20}$ with values sorted by mole\%. T2G-34 was fabricated from oxide versions of the chloride waste components in which the same total waste component cations (Cat.) were identical to the equivalent glass batched with the chloride components, T2G-22, thus T2G-22 serves as a reference for this study. Thus, the ratios of Te:Cat and $\mathrm{Pb}$ :Cat were fixed at 6.16 and 1.24 , respectively. As with the other lead-tellurite glasses, the $\mathrm{Pb}$ :Te ratio for these glasses was fixed at 0.202 .

\begin{tabular}{|c|c|c|c|c|c|}
\hline Glass ID: & T2G-22 & T2G-34 & Component & Mole $\%$ & Mass\% \\
\hline & \multicolumn{2}{|c|}{ Mass \% } & $\mathrm{Li}_{2} \mathrm{O}$ & 46.5 & 19.09 \\
\hline$\overline{\mathrm{TeO}_{2}}$ & 74.1 & 74.6 & $\mathrm{~K}_{2} \mathrm{O}$ & 33.6 & 43.48 \\
\hline $\mathrm{PbO}$ & 20.9 & 21.0 & $\mathrm{Na}_{2} \mathrm{O}$ & 13.2 & 11.25 \\
\hline$X \mathrm{Cl}_{20}$ & 5.0 & & $\mathrm{Cs}_{2} \mathrm{O}$ & 1.53 & 5.93 \\
\hline \multirow[t]{2}{*}{$X \mathrm{O}_{20}$} & & 4.4 & $\mathrm{Nd}_{2} \mathrm{O}_{3}$ & 1.41 & 6.51 \\
\hline & \multicolumn{2}{|c|}{ Mole\% } & $\mathrm{BaO}$ & 1.27 & 2.68 \\
\hline $\mathrm{TeO}_{2}$ & 73.3 & 77.9 & $\mathrm{Ce}_{2} \mathrm{O}_{3}$ & 0.940 & 4.24 \\
\hline $\mathrm{PbO}$ & 14.8 & 15.7 & $\mathrm{La}_{2} \mathrm{O}_{3}$ & 0.617 & 2.76 \\
\hline$X \mathrm{Cl}_{20}$ & 11.9 & & $\mathrm{Pr}_{2} \mathrm{O}_{3}$ & 0.458 & 2.08 \\
\hline$X \mathrm{O}_{20}$ & & 6.4 & $\mathrm{Sm}_{2} \mathrm{O}_{3}$ & 0.414 & 1.98 \\
\hline$\overline{M(\mathrm{~Pb}: \mathrm{Te})}$ & 0.202 & 0.202 & & & \\
\hline$M$ (Te:Cat.) & 6.16 & 6.16 & & & \\
\hline$M$ (Pb:Cat.) & 1.24 & 1.24 & & & \\
\hline
\end{tabular}


Set 4: $\mathbf{P b O}-\mathbf{K C l} / \mathbf{K}_{2} \underline{O}$ and Set 5: $\mathbf{Z n O}-\mathbf{K C l} / \mathbf{K}_{2} \underline{O}$. The purpose of these studies was to compare the structural differences between $\mathrm{PbO} / \mathrm{ZnO}$ glasses with additions of potassium as either a chloride or an oxide. The purpose of adding potassium as either $\mathrm{KCl}$ or $\mathrm{K}_{2} \mathrm{O}$ was to determine the anion effect on the structure. For each of these sets $(\mathrm{PbO}$ or $\mathrm{ZnO})$, the ratios between the metal additive and $\mathrm{TeO}_{2}$ were kept constant from the original $\mathrm{P}$-series glasses and the $\mathrm{K}: \mathrm{Te}$ and $\mathrm{K}: \mathrm{M}$ (where $\mathrm{M}=\mathrm{Pb}, \mathrm{Zn}$ ) ratios were also kept constant for each system. The $\mathrm{PbO}(\mathrm{P}-8$ and $\mathrm{P}-11)$ and $\mathrm{ZnO}(\mathrm{P}-4$ and $\mathrm{P}-13)$ glasses performed very differently during the PCT and this study was designed to investigate the possibility of structural differences in these more simple glass formulations (Table 2.6). Compositions for the $\mathrm{PbO}$ and $\mathrm{ZnO}$ glasses are presented in Table 3.10 and Table 3.11, respectively.

Table 3.10. Compositions for $\mathrm{T} 2 \mathrm{G}-27 \mathrm{a}, \mathrm{T} 2 \mathrm{G}-27 \mathrm{~b}, \mathrm{~T} 2 \mathrm{G}-29 \mathrm{a}$, and $\mathrm{T} 2 \mathrm{G}-29 \mathrm{~b}$ anion variation glasses (in mole\%) as well as the mass ratio of $\mathrm{TeO}_{2}: \mathrm{PbO}$. Here, $m$ and $M$ denote ratios by mass and moles, respectively. The baseline $\mathrm{P}$-series glasses for this variation study, P-8 and P-4, are listed in bold type.

\begin{tabular}{lccc}
\hline Glass ID: & $\mathbf{P - 8}$ & $\mathrm{T} 2 \mathrm{G}-27 \mathrm{a}$ & $\mathrm{T} 2 \mathrm{G}-27 \mathrm{~b}$ \\
\hline $\mathrm{TeO}_{2}$ & $\mathbf{8 3 . 2 2}$ & 76.47 & 70.73 \\
$\mathbf{P b O}$ & $\mathbf{1 6 . 7 8}$ & 15.42 & 14.27 \\
$\mathrm{~K}_{2} \mathrm{O}$ & - & 8.11 & - \\
$\mathrm{KCl}$ & - & - & 15.00 \\
\hline$m\left(\mathrm{TeO}_{2}: \mathbf{P b O}\right)$ & $\mathbf{3 . 5 5}$ & 3.55 & 3.55 \\
\hline$M(\mathbf{P b}: \mathrm{Te})$ & $\mathbf{0 . 2 0 2}$ & 0.202 & 0.202 \\
$M(\mathrm{~K}: \mathrm{Te})$ & - & 0.212 & 0.212 \\
$M(\mathrm{~K}: \mathbf{P b})$ & - & 1.05 & 1.05 \\
\hline
\end{tabular}

Table 3.11. Compositions for $\mathrm{T} 2 \mathrm{G}-29 \mathrm{a}$ and $\mathrm{T} 2 \mathrm{G}-29 \mathrm{~b}$ anion variation glasses (in mole $\%$ ) as well as the mass ratio of $\mathrm{TeO}_{2}: \mathrm{PbO}$. Here, $m$ and $M$ denote ratios by mass and moles, respectively. The baseline $\mathrm{P}-$ series glasses for this variation study, P-8 and P-4, are listed in bold type.

\begin{tabular}{lccc}
\hline Glass ID: & P-4 & T2G-29a & T2G-29b \\
\hline $\mathrm{TeO}_{2}$ & $\mathbf{6 9 . 9 1}$ & 66.23 & 62.92 \\
$\mathrm{ZnO}$ & $\mathbf{3 0 . 0 9}$ & 28.51 & 27.08 \\
$\mathrm{~K}_{2} \mathrm{O}$ & - & 5.26 & - \\
$\mathrm{KCl}$ & - & - & 10.00 \\
\hline$m\left(\mathrm{TeO}_{2}: \mathrm{ZnO}\right)$ & $\mathbf{4 . 5 6}$ & 4.56 & 4.56 \\
\hline$M(\mathrm{Zn}: \mathrm{Te})$ & $\mathbf{0 . 4 3 0}$ & 0.430 & 0.430 \\
$M(\mathrm{~K}: \mathrm{Te})$ & - & 0.159 & 0.159 \\
$M(\mathrm{~K}: \mathrm{Zn})$ & - & 0.369 & 0.369 \\
\hline
\end{tabular}

\subsection{Tellurite Crystal Fabrication}

We selected twelve tellurite crystals to fabricate from the list put forth by Sakida and coworkers (1999a), and they are listed in Table 3.12. The list we selected included a few of the crystals from each category (see Figure 1.1). We made a few assumptions regarding the methods for fabricating these crystals based on the methods proposed by Sakida et al. because of missing details in the published methods. The assumptions and comments are as follows:

- Sakida et al. did mention that they had melted (M) the reactants in Table 3.12 at a particular temperature but they did not report the dwell time for the melt at that temperature. Therefore, we assumed the dwell time of $1 \mathrm{~h}$ to be sufficient for this process. Further, they either cooled the 
melt to room temperature $(\mathbf{R T})$ or quenched $(\mathbf{Q U})$ it but did not report the cooling rate. In this context, we assumed that a cooling rate of $5{ }^{\circ} \mathrm{C} / \mathrm{min}$ should be sufficient.

- Sakida et al. did not list the crucible type that they used. We assumed that a platinum alloy crucible and lid would be sufficient.

- Sakida et al. added small quantities of $\mathrm{Fe}_{2} \mathrm{O}_{3}(0.3 \mathrm{~mole} \%)$ to their batches to improve the NMR signal to noise ratio by shortening the relaxation times of Te nuclei with $I=1 / 2$. We also did this.

Table 3.12. Tellurite crystals selected for fabrication at PNNL from the list proposed by Sakida et al. (1999a). Multiple steps are numbered for some of the crystals. Crystals in bold and italics show similar amorphous structure for the $\mathrm{TeO}_{3}$-isolated and $\alpha-\mathrm{TeO}_{2}$ units, respectively (see Figure 3.2). The methods listed here were those that proved successful by Sakida et al. "HT" stands for heat-treatment. Note: All crystals listed here also have an additional $0.3 \mathrm{~mol} \% \mathrm{Fe}_{2} \mathrm{O}_{3}$ added to shorten the relaxation time for the Te nuclei with $I=1 / 2$ during NMR analysis.

\begin{tabular}{|c|c|c|c|c|}
\hline Type & Crystal & Starting Materials & $\begin{array}{l}\text { Temp. }\left({ }^{\circ} \mathrm{C}\right) / \mathrm{HT} \text { time } \\
\text { (h) }\end{array}$ & $\begin{array}{l}\text { Powder Diffraction File } \\
\text { (PDF) Reference }\end{array}$ \\
\hline \multirow{4}{*}{$\begin{array}{l}\mathrm{TeO}_{3-}^{-} \\
\text {isolated }\end{array}$} & $\mathrm{PbTeO}_{3}$ & $\mathrm{TeO}_{2}, \mathrm{PbO}, \mathrm{Fe}_{2} \mathrm{O}_{3}$ & $\begin{array}{l}\text { (1) } 850(\mathrm{M}) / 1 \rightarrow \text { RT } \\
\text { (2) } 450(\mathbf{1 0}) \rightarrow \text { RT }\end{array}$ & (Sciau et al., 1986) \\
\hline & $\mathrm{Li}_{2} \mathrm{TeO}_{3}$ & $\mathrm{TeO}_{2}, \mathrm{Li}_{2} \mathrm{CO}_{3}, \mathrm{Fe}_{2} \mathrm{O}_{3}$ & $850(\mathrm{M}) / 1 \rightarrow \mathbf{R T}$ & (Folger, 1975a) \\
\hline & $\mathrm{ZnTeO}_{3}$ & $\mathrm{TeO}_{2}, \mathrm{ZnO}, \mathrm{Fe}_{2} \mathrm{O}_{3}$ & $\begin{array}{l}\text { (1) } 1200(\mathrm{M}) / 1 \rightarrow \mathrm{QU} \\
\text { (2) } 650(\mathbf{6}) \rightarrow \mathbf{R T}\end{array}$ & (Hanke, 1967) \\
\hline & $\mathrm{BaTeO}_{3}$ & $\mathrm{TeO}_{2}, \mathrm{BaCO}_{3}, \mathrm{Fe}_{2} \mathrm{O}_{3}$ & $1200(\mathrm{M}) / 1 \rightarrow \mathbf{R T}$ & (Folger, 1975b) \\
\hline \multirow{2}{*}{$\begin{array}{l}\mathrm{TeO}_{3-} \\
\text { terminal }\end{array}$} & $\mathrm{Cs}_{2} \mathrm{Te}_{2} \mathrm{O}_{5}$ & $\mathrm{TeO}_{2}, \mathrm{Cs}_{2} \mathrm{CO}_{3}, \mathrm{Fe}_{2} \mathrm{O}_{3}$ & $550(\mathrm{M}) \rightarrow \mathbf{R T}$ & $\begin{array}{l}\text { (Loopstra and Goubitz, } \\
\text { 1986) }\end{array}$ \\
\hline & $\mathrm{Te}_{2} \mathrm{~V}_{2} \mathrm{O}_{9}$ & $\mathrm{TeO}_{2}, \mathrm{~V}_{2} \mathrm{O}_{5}, \mathrm{Fe}_{2} \mathrm{O}_{3}$ & $\begin{array}{l}\text { (1) } 650(\mathrm{M}) / 1 \rightarrow \text { RT } \\
\text { (2) } 430(\mathbf{1 2}) \rightarrow \text { RT }\end{array}$ & (Darriet and Galy, 1973) \\
\hline \multirow{2}{*}{$\mathrm{TeO}_{3+1}$} & $\mathrm{Zn}_{2} \mathrm{Te}_{3} \mathrm{O}_{8}$ & $\mathrm{TeO}_{2}, \mathrm{ZnO}, \mathrm{Fe}_{2} \mathrm{O}_{3}$ & $\begin{array}{l}\text { (1) } 850(\mathrm{M}) / 1 \rightarrow \mathrm{QU} \\
\text { (2) } 650(7) \rightarrow \mathbf{R T}\end{array}$ & (Hanke, 1966) \\
\hline & $\mathrm{Mg}_{2} \mathrm{Te}_{3} \mathrm{O}_{8}$ & $\mathrm{TeO}_{2}, \mathrm{MgO}, \mathrm{Fe}_{2} \mathrm{O}_{3}$ & $\begin{array}{l}\text { (1) } 1200(\mathrm{M}) / 1 \rightarrow \mathrm{QU} \\
\text { (2) } 650(7) \rightarrow \mathbf{R T}\end{array}$ & $\begin{array}{l}\text { (Tromel and Ziethen- } \\
\text { Reichnach, 1970) }\end{array}$ \\
\hline \multirow{3}{*}{$\alpha-\mathrm{TeO}_{2}$} & $\mathrm{SnTe}_{3} \mathrm{O}_{8}$ & $\mathrm{TeO}_{2}, \mathrm{SnO}_{2}, \mathrm{Fe}_{2} \mathrm{O}_{3}$ & $650(\mathbf{8 5}) \rightarrow \mathbf{R T}$ & (Meunier and Galy, 1971) \\
\hline & $\mathrm{ZrTe}_{3} \mathrm{O}_{8}$ & $\mathrm{TeO}_{2}, \mathrm{ZrO}_{2}, \mathrm{Fe}_{2} \mathrm{O}_{3}$ & $650(\mathbf{6 5}) \rightarrow \mathbf{R T}$ & (Meunier and Galy, 1971) \\
\hline & $\mathrm{TiTe}_{3} \mathrm{O}_{8}$ & $\mathrm{TeO}_{2}, \mathrm{TiO}_{2}, \mathrm{Fe}_{2} \mathrm{O}_{3}$ & $650(\mathbf{8 5}) \rightarrow \mathbf{R T}$ & (Meunier and Galy, 1971) \\
\hline$\beta-\mathrm{TeO}_{2}$ & $\mathrm{NaVTeO}_{5}$ & $\begin{array}{l}\mathrm{TeO}_{2}, \mathrm{Na}_{2} \mathrm{CO}_{3}, \mathrm{~V}_{2} \mathrm{O}_{5}, \\
\mathrm{Fe}_{2} \mathrm{O}_{3}\end{array}$ & $\begin{array}{l}\text { (1) } 600(\mathrm{M}) / 1 \rightarrow \mathrm{RT} \\
\text { (2) } 500(\mathbf{4}) \rightarrow \mathbf{R T}\end{array}$ & (Darriet et al., 1972) \\
\hline
\end{tabular}

M: melting (time $=1-2 \mathrm{~h}$ )

RT: room temperature with a cooling rate of $1^{\circ} \mathrm{C} / \mathrm{min}$ )

QU: quenched 


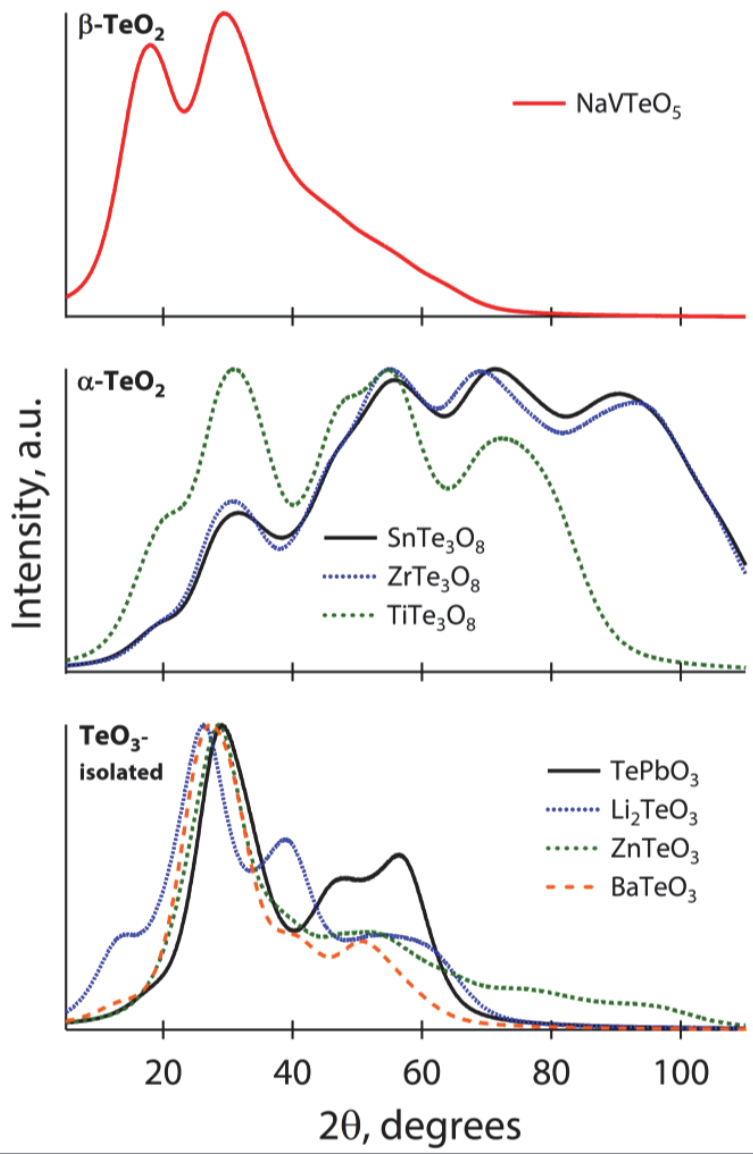

Figure 3.2. Simulated amorphous patterns of a few tellurite crystals. These patterns were generated in JADE by setting the crystallite size to $10 \AA$ and putting $0 \%$ strain on the network. These were used to predict the amorphous structure of the crystalline compounds.

\subsection{Waste Form Characterization}

\subsubsection{Optical Microscopy and Photo-Documentation}

Specimen microstructure was analyzed with Olympus SZH10 and Olympus PMG-3 optical microscopes. In some cases, polished cross-section of the glasses were observed in reflected or transmitted light, depending on the opacity of the specimen, but most of the time, the glasses were observed as-quenched. Low-magnification optical micrographs and photographs (both in color) were collected of the bulk glasses, of the crucible, and of the pour plate surface for process comparisons between compositions, with a Canon T1i camera (Canon U.S.A., Inc., Lake Success, NY).

\subsubsection{Helium Pycnometry - Density Measurements for Tellurite Glasses}

The density of each tellurite glass for PCT measurements was measured with a Micromeritics AccuPyc II 1340 helium gas pycnometer. The pycnometer was configured to perform five purges and ten measurements during calibration and specimen analysis. The pycnometer was calibrated using a standard of known volume and the equilibrium rate was $0.0050 \mathrm{psig} / \mathrm{min}$. Mass values were measured with an analytical balance $( \pm 0.0003$ precision). For all of these measurements, the entire specimen was placed into the $10 \mathrm{~cm}^{3}$ sample chamber - this allowed for a more representative measured density of the entire specimen. 


\subsubsection{Density and Porosity Measurements for High-Halide Minerals}

Before firing, the green bulk density $\left(\rho_{\mathrm{g}}\right)$ was measured from the pellet weight $( \pm 0.3 \mathrm{mg})$ and dimensions $( \pm 0.01 \mathrm{~mm})$. After firing, the fired bulk density $\left(\rho_{\mathrm{b}}\right)$ was measured. Then, the specimens were broken into quadrants for characterization. Archimedes' method was used with ethanol to measure the density $\left(\rho_{\mathrm{a}}\right)$ with Equation (6) where $m_{\text {dry }}, m_{\text {sub }}$, and $m_{\text {sat }}$, are the masses (in $\mathrm{g}$ ) of the specimen dry, submersed in ethanol, and saturated in ethanol, respectively, and the density of ethanol $\left(\rho_{\mathrm{e}}\right)$ was calculated according to Stratton (1924). Also, the open porosity $\left(\phi_{0}\right)$ was measured with Equation (7).

$$
\begin{gathered}
\rho_{\mathrm{a}}=\left(m_{\mathrm{dry}} * \rho_{\mathrm{e}}\right) /\left(m_{\mathrm{sat}}-m_{\text {sub }}\right) \\
\phi_{\mathrm{o}}=100 *\left(m_{\text {sat }}-m_{\text {dry }}\right) /\left(m_{\text {sat }}-m_{\text {sub }}\right)
\end{gathered}
$$

The theoretical density, $\rho_{\mathrm{t}}$, of the fired pellets without any porosity was calculated with Equation (8) where $\rho_{\mathrm{i}}$ is the density and $m_{\mathrm{i}}$ is the mass fraction (unitless) of the $i$-th component determined with X-ray diffraction (XRD). This corrects for phases of different densities when calculating the overall density. However, some error is associated with this approach considering that the $\rho_{\mathrm{i}}$ values used were provided

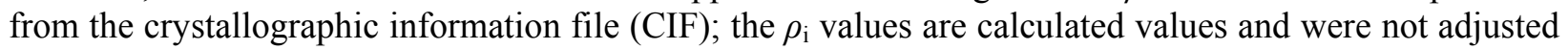
for compositional differences between the phases observed with XRD and those presented in the CIF. Then, the total porosity $\left(\phi_{\mathrm{t}}\right)$ was calculated with Equation (9) where the term " $\left(\rho_{\mathrm{a}}+\rho_{\mathrm{b}}\right) / 2$ " is the average measured density from the Archimedes $\left(\rho_{\mathrm{a}}\right)$ and bulk $\left(\rho_{\mathrm{b}}\right)$ techniques.

$$
\begin{gathered}
\rho_{\mathrm{t}}=\sum_{\mathrm{i}=1}^{n} p_{\mathrm{i}} m_{\mathrm{i}} \\
\phi_{\mathrm{t}}=100 *\left[1-\left(\frac{\rho_{\mathrm{a}}+\rho_{\mathrm{b}}}{2 * \rho_{\mathrm{t}}}\right)\right]
\end{gathered}
$$

\subsubsection{Storage density}

Measured density was then combined with the waste loading for each specimen and the storage density, $\rho_{\text {sd }}$, calculated with Equation (10). Storage density $\left(\rho_{\text {sd }}\right)$ is defined as the mass of waste per volume of waste package, where "waste package" denotes the glass with immobilized waste.

$$
\rho_{\text {sd }}=\frac{\text { mass waste }(\mathrm{kg})}{\text { volume of waste package }\left(\mathrm{m}^{3}\right)}
$$

\subsubsection{X-Ray Diffraction}

Specimens of both the sodalites and glasses were analyzed with an XRD in powder form. These powders were prepared in a tungsten carbide milling chamber (minerals) or with a mortar and pestle (glasses). The XRD used for structure and phase analysis was a Bruker D8 Advanced equipped with a $\mathrm{Cu} \mathrm{K} \alpha$ target set to a power level of $40 \mathrm{kV}$ and $40 \mathrm{~mA}$. The detector used was a position-sensitive detector with an angle collection range of $3^{\circ} 2 \theta$. Typical scan parameters used for specimen analysis were $5-110^{\circ} 2 \theta$ for the tellurite compounds and 5-70 $2 \theta$ for the high-halide minerals with a step of $0.015^{\circ} 2 \theta$ and a 0.3 second dwell at each step. $\mathrm{JADE}^{\mathrm{TM}}$ 6, Bruker $\mathrm{AXS}^{\mathcal{O}}$ EVA, and Topas 4.2 software were used to identify and quantify phase assemblages.

\subsubsection{Raman Spectroscopy}

Raman spectra were collected on powdered glasses with a SPEX 1877 Triplemate spectrometer (Horiba JY, Edison, NJ) equipped with a liquid nitrogen-cooled silicon charge-coupled device detector (Princeton Instruments, Trenton, NJ) and the $514.5 \mathrm{~nm}$ line of a Coherent (Santa Clara, CA) Innova $307 \mathrm{Ar}$ ion laser for excitation. Data were collected in backscatter geometry with a $400 \mu \mathrm{m}$ slit with approximately $5 \mathrm{~mW}$ of laser power at the specimen and a $100 \mathrm{~s}$ acquisition time. The estimated uncertainty of the peak frequencies was $\pm 1 \mathrm{~cm}^{-1}$ for all of the Raman spectra.

Quantitative spectral analysis and curve fitting of the Raman data was performed using Thermo Fisher Scientific (Waltham, MA) Grams/32 AI software, which uses the Levenburg-Marquardt nonlinear peak 
fitting method (Levenberg, 1944). Prior to curve fitting, a decaying exponential baseline correction was used to remove the background due to Rayleigh scattering. The remaining spectrum in the $300-1500 \mathrm{~cm}^{-1}$ region was fitted, giving results with $R^{2} \geq 0.998$.

Several protocols were considered for quantitative fitting of the Raman spectra. First, peak fitting was performed with no constraints and trends compared. This seemed to work satisfactorily with the compositional variation study glasses where a series of bands were identified and coordinated with literature values. However, for the lead-tellurite and structural variation study glasses, this method was deemed unsatisfactory, so a subsequent set of fits were performed with "fixed" wavenumber positions determined from the optimum positions for the $\mathrm{TPb}_{0}$ glass for the main Raman bands in that glass. The frequencies, assignments, and literature references for these bands are shown in Table 3.13. The fixed frequency approach yielded $R^{2}$ fit values better than 0.999 . For the purposes of analysis, ratios of peak areas were compared as a function of $X \mathrm{Cl}_{20}$-loading or $\mathrm{Cl} / \mathrm{O}$ ratio.

For the P-series glasses, the Raman fitting was performed with no fixed parameters, and the peak frequencies of the bands were allowed to float as well as the widths as opposed to fixing the frequencies and just allowing the widths to float, as we did subsequently. As mentioned above, the latter method with fixed frequencies appeared to result in more consistent results when the $\mathrm{TPb}_{\#}$ glasses were analyzed as a function of composition change. The fixed Raman frequency bands that we used for quantitative analysis of the $\mathrm{TPb}_{\#}$ glasses are presented in Table 3.13 where the "E1/E2" bands were combined into "E" for the compositional variation study but were deconvolved into $\mathbf{E 1}$ and $\mathbf{E 2}$ for the rest of the glasses.

In order to determine the structural differences between the two phases present in the $15 \% X \mathrm{Cl}_{20}$-loaded glass, both macro- and micro-Raman spectra were collected on each phase from this bulk piece. Raman spectra were obtained with the same spectrometer and laser $(514.5 \mathrm{~nm})$ as used in the macro measurements. However, the laser beam was coupled to a confocal Olympus BH2 microscope (Center Valley, PA) equipped with an $80 \times$ objective and numerical aperture equal to 0.75 . Laser power at the specimen was approximately $10 \mathrm{~mW}$. The slit width was $400 \mu \mathrm{m}$ and the exposure time was $100 \mathrm{~s}$ for all specimens.

\subsubsection{Chemical Durability with the Product Consistency Test (PCT)}

The chemical durability test performed was the PCT, a seven-day static leaching test in deionized water at $90{ }^{\circ} \mathrm{C}$. The PCT was performed on each of the glasses according to Test Method B (PCT-B) of the ASTM International Standard C 1285-02. The chemical durability is characterized quantitatively by the normalized sodium release $\left(N L_{\mathrm{Na}}\right.$, in $\left.\mathrm{g} / \mathrm{m}^{2}\right)$, being higher for less chemically durable glasses. Detailed methods and results were described in the FY2010 report (Riley et al., 2010).

For the sodalite samples, the specific surface areas of powders sieved to $75-152 \mu \mathrm{m}$ were measured with the Brunauer-Emmett-Teller (BET) method (Brunauer et al., 1938) on $\mathrm{N}_{2}(\mathrm{~g})$ adsorption and desorption isotherms collected with an Autosorb-6B (Quantachrome Instruments, Boynton Beach, FL). The mass of specimen used was equivalent to the entire sieved fraction obtained for chemical durability tests with the product consistency test (ASTM C 1285-08, 2008). A helium pycnometer was used to measure specimen density with an AccuPyc II 1340 (Micromeritics Instrument Corporation, Norcross, GA).

\subsubsection{Thermal Analysis (DTA, TGA, DSC)}

The P-series lead-tellurite glasses were analyzed with a TA Instruments SDT Q600 (New Castle, DE) differential thermal-thermogravimetric analyzer (DTA-TGA). Here, specimens were run at various ramp rates (e.g., $10^{\circ} \mathrm{C}, 15^{\circ} \mathrm{C}$ ) to determine the effect of ramp rate on the glass transition temperature $\left(T_{\mathrm{g}}\right)$, onset crystallization temperature $\left(T_{\mathrm{x}}\right)$, crystallization temperature $\left(T_{\mathrm{c}}\right)$, and $T_{\mathrm{M}}$.

Differential scanning calorimetry (DSC) and TGA were performed on the powder of each glass from the lead tellurite series with a Jupiter ${ }^{\circledR}$ STA 449C (NETZSCH Instruments N.A. LLC, Burlington, MA). For the DSC/TGA experiments, specimens were loaded into alumina $\left(\mathrm{Al}_{2} \mathrm{O}_{3}\right)$ pans, without lids, placed onto a 
DSC/TG High RG4 sample carrier, and heated at $10{ }^{\circ} \mathrm{C} / \mathrm{min}$ from room temperature $\left(\sim 25{ }^{\circ} \mathrm{C}\right)$ to $750{ }^{\circ} \mathrm{C}$ with air as the carrier gas at a flow rate of $50 \mathrm{~mL} / \mathrm{min}$. The spectra were analyzed with Proteus ${ }^{\circledR}$ software (v 5.3.0/24.05.2011, NETZSCH Instruments North America, LLC, Burlington, MA).

The DTA and DSC results were analyzed and the glass-formation ability $\left(K_{\mathrm{gl}}\right)$ was calculated according to the Hruby criterion — see Equation (11) — where $T_{\mathrm{c}}, T_{\mathrm{g}}$, and $T_{\mathrm{M}}$ are the crystallization, glass transition, and melting temperatures of the glass (Hruby, 1972).

$$
K_{\mathrm{gl}}=\left(T_{\mathrm{c}}-T_{\mathrm{g}}\right) /\left(T_{\mathrm{M}}-T_{\mathrm{g}}\right)
$$

Since the DSC data at temperatures above $500{ }^{\circ} \mathrm{C}$ did not appear reliable, the $T_{\mathrm{M}}$ could not be determined, accurately. Thus, we used a modified version of the Hruby criterion, presented by Wang et al., to compare the glass-formation ability with only the numerator from Equation (9), $T_{\mathrm{c}}-T_{\mathrm{g}}$ (Wang et al., 1994). This difference provides an estimate of how difficult it would be to quench from a melt and prevent crystallization before reaching the $T_{\mathrm{g}}$.

Additionally, thermal diffusivity was measured for P-11 and Sodalite S5A with a NETZSCH Laser Flash Apparatus 457 (LFA). For the sodalites, powders of Sodalite 5A were pressed into a pellet, and fired at $850^{\circ} \mathrm{C}$. Then, two 12.5 -mm diameter discs were core-drilled out of the pellets that were $\sim 2$-mm thick. The glass specimens for thermal diffusivity measurements were discs cut from cylinders made by pourcasting the glasses into fused silica molds at the $T_{\mathrm{M}}$ of the glass (Figure 3.3). Thus, the glasses were melted, poured into cylindrical quartz molds and annealed at $240{ }^{\circ} \mathrm{C}$ for $4 \mathrm{~h}$, after which the annealing furnace was allowed to air-cool to room temperature. These cylinders were then cut into discs with a wire saw and polished to a thickness of $\sim 2-3 \mathrm{~mm}$.

The specimens were coated with Graphit ${ }^{\circledR}$ spray provided by NETZSCH, which was applied to help make sure that all of the LFA laser energy was absorbed at the front face of the specimen. Measurements for the sodalite specimens were collected at room temperature and then every $100{ }^{\circ} \mathrm{C}$ between $100{ }^{\circ} \mathrm{C}$ and $700{ }^{\circ} \mathrm{C}$. The disc of the P-11 glass was measured at $20^{\circ} \mathrm{C}$ and then in $50^{\circ} \mathrm{C}$ increments from 50 to $250^{\circ} \mathrm{C}$, terminating near the $T_{\mathrm{g}}$ of the material. Five data points were collected at each temperature and averaged.

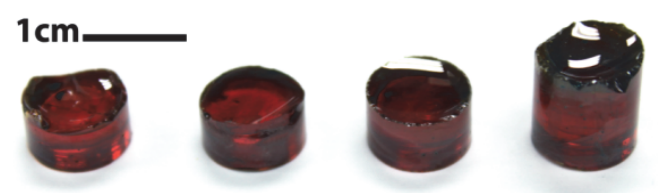

Figure 3.3. Cast cylinders of P-11 glass from which discs were cut for the thermal diffusivity measurements.

\subsubsection{Scanning Electron Microscopy and Energy Dispersive Spectroscopy (SEM-EDS)}

Specimens were observed in greater detail with scanning electron microscopy (SEM) and energy dispersive spectroscopy (EDS) using a JSM-5900 with a tungsten filament and a JSM-7001F with a field emission gun (JEOL, Ltd., Tokyo, Japan). For both of these systems, an EDAX Apollo II $30 \mathrm{~mm}^{2} \mathrm{Si}$ drift EDS detector (AMETEK, Berwyn, PA) was used for compositional analysis. Specimens were analyzed coated with palladium on the JSM-5900 and analyzed both coated and uncoated on the JSM-7001F. Both secondary electron (SEI) and backscattered electron (BSE) detectors were used for imaging.

\subsubsection{Transmission Electron Microscopy and Energy Dispersive Spectroscopy (TEM)}

Analytical transmission electron microscopy (ATEM) was performed on a JEOL 2010F field-emission gun microscope operated at $200 \mathrm{kV}$ using both 20 - and $50-\mu \mathrm{m}$ diameter condenser apertures. Compositional analyses and elemental mapping were obtained with an Oxford INCA EDS (Oxford 
Instruments, Concord, MA) with a fine-probe $(0.7 \mathrm{~nm}$ diameter $)$ in scanning TEM (STEM) mode. Powders of each glass studied were passed through a 325- $\mu \mathrm{m}$ mesh sieve, dispersed in isopropanol, and crushed in an agate mortar and pestle. Lacey carbon copper grids were then passed through the solution and allowed to dry. Regions of interest were identified at electron transparent edges of the crushed powders with a $20-\mu \mathrm{m}$ condenser aperture so as to avoid inducing phase separation through beam damage. After analysis, regions of interest were further analyzed with the $50-\mu \mathrm{m}$ aperture as to increase beam intensity on the specimen if allowable. Bright field (BF), selected area diffraction (SAD), and dark field (DF) imaging were used in normal TEM mode. 


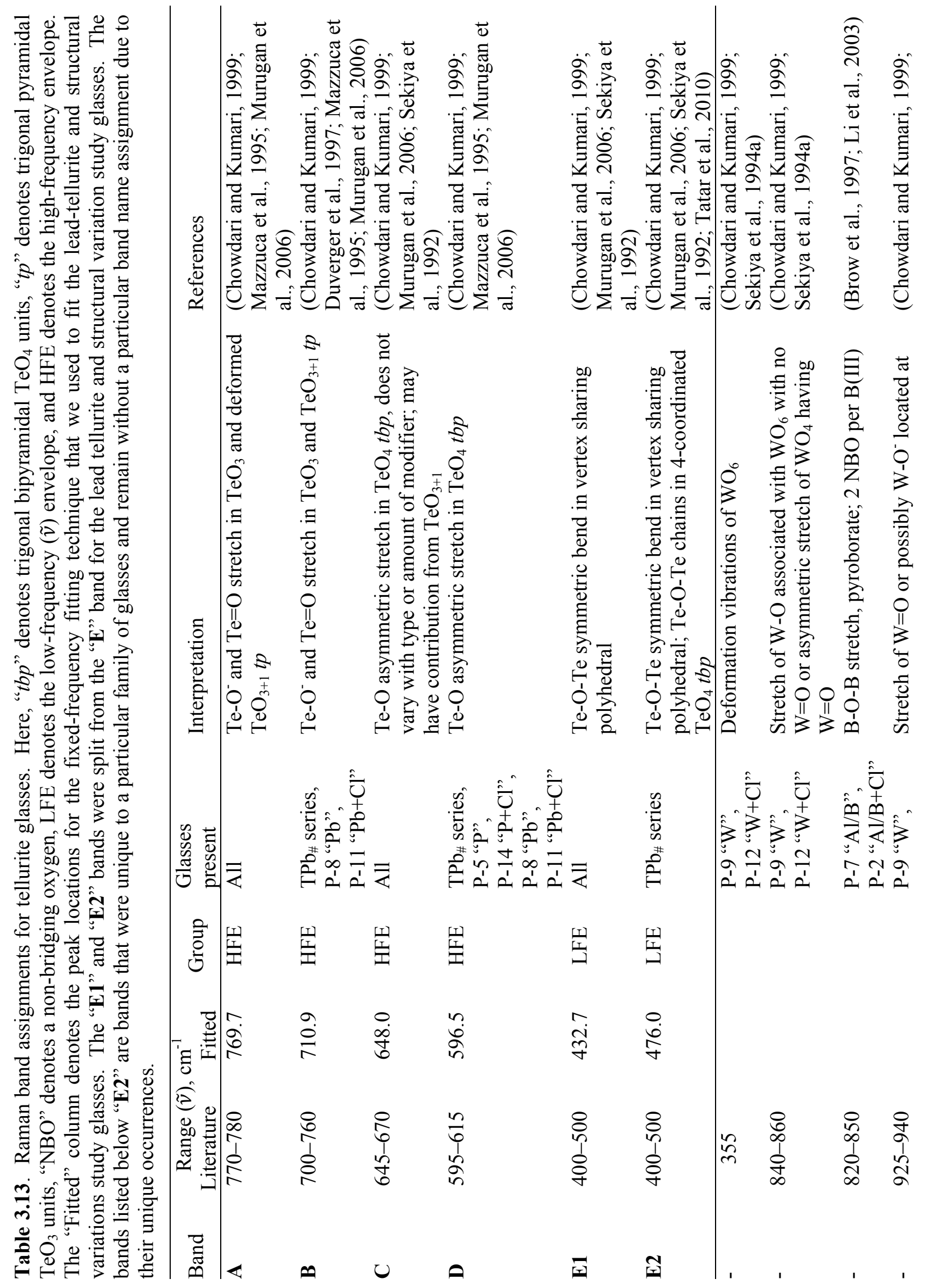




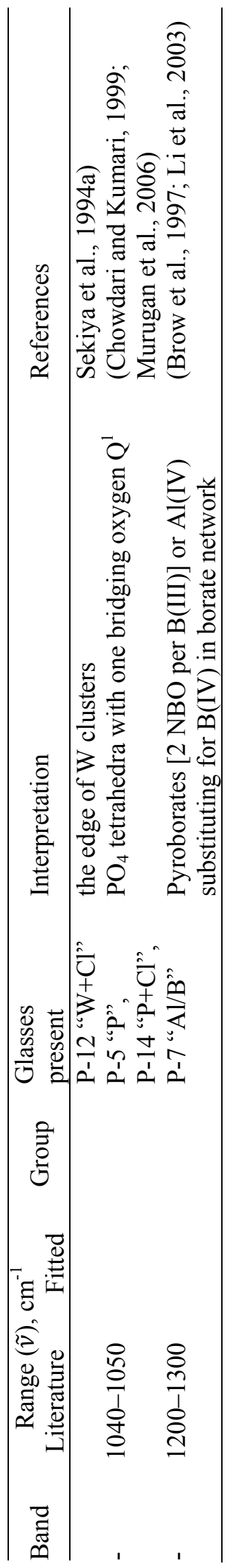




\subsubsection{Nuclear Magnetic Resonance (NMR)}

Nuclear magnetic resonance was performed on select glasses for ${ }^{23} \mathrm{Na},{ }^{125} \mathrm{Te},{ }^{207} \mathrm{~Pb}$, and ${ }^{35} \mathrm{Cl}$. Experiments for ${ }^{23} \mathrm{Na}$ were performed at $21.14 \mathrm{~T}\left(900 \mathrm{MHz}\right.$ for ${ }^{1} \mathrm{H}$ and $238.088 \mathrm{MHz}$ for $\left.{ }^{23} \mathrm{Na}\right)$ in a medium-bore (63 $\mathrm{mm}$ ) Oxford magnet utilizing a Varian/Agilent NMR systems (VNMRS) console and an Agilent $3.2 \mathrm{~mm}$ MAS probe. The ${ }^{23} \mathrm{Na}$ direct polarization data were acquired utilizing a spinning speed of $15 \mathrm{kHz}$, a $\pi / 20$ pulse of $0.30 \mu \mathrm{s}$, and a recycle delay of $1 \mathrm{~s}$. The number of transients acquired varied from 964 to 9764 , depending upon the amount of sodium in the specimen. Each individual time domain signal was apodized with $200 \mathrm{~Hz}$ of Lorentzian line broadening. Chemical shifts were referenced with respect to $0.5 \mathrm{M} \mathrm{NaCl}(\mathrm{aq})$ at $0 \mathrm{ppm}$.

The ${ }^{125} \mathrm{Te},{ }^{207} \mathrm{~Pb}$, and ${ }^{35} \mathrm{Cl}$ experiments were performed at $9.4 \mathrm{~T}\left(399.9 \mathrm{MHz}\right.$ for ${ }^{1} \mathrm{H}, 126.362 \mathrm{MHz}$ for ${ }^{125} \mathrm{Te}, 83.664 \mathrm{MHz}$ for ${ }^{207} \mathrm{~Pb}$, and $39.182 \mathrm{MHz}$ for ${ }^{35} \mathrm{Cl}$ ) in a wide-bore $(89 \mathrm{~mm}$ ) Oxford magnet utilizing a Varian/Agilent Inova console and a Doty $5 \mathrm{~mm}$ wideline probe. Direct polarization was utilized for the acquisition, with a $\pi / 2$ pulse of $4 \mu \mathrm{s}\left({ }^{125} \mathrm{Te}\right), 4.5 \mu \mathrm{s}\left({ }^{207} \mathrm{~Pb}\right)$, or $3.7 \mu \mathrm{s}\left({ }^{35} \mathrm{Cl}\right)$, a recycle delay of $30 \mathrm{~s}\left({ }^{35} \mathrm{Cl}\right)$ or $120 \mathrm{~s}\left({ }^{125} \mathrm{Te}\right.$ and $\left.{ }^{207} \mathrm{~Pb}\right)$ with signal detection utilizing a quadrupole Carr-Purcell-Meiboom-Gill (QCPMG) echo train (Larsen et al., 1997; Larsen et al., 1999). Due to the width of the lineshapes, the entire dataset could not be acquired in a single experiment so the offset frequency was stepped every 20 $\mathrm{kHz}$ and then spectra from all of the offsets were combined in a sky projection to construct the final spectrum (Lipton et al.). Each individual time domain signal was apodized with a comb filter (Lipton et al.) $\left(5 \mathrm{kHz}\right.$ for ${ }^{125} \mathrm{Te}$ and ${ }^{207} \mathrm{~Pb}$ and $2.5 \mathrm{kHz}$ for $\left.{ }^{35} \mathrm{Cl}\right)$ matched to the echo time followed by $10 \mathrm{~Hz}\left({ }^{125} \mathrm{Te}\right.$, $\left.{ }^{35} \mathrm{Cl}\right)$ or $25 \mathrm{~Hz}\left({ }^{207} \mathrm{~Pb}\right)$ of Lorentzian line broadening. Chemical shifts were referenced with respect to $0.69 \mathrm{M} \mathrm{Te}(\mathrm{OH})_{6}$ at $0 \mathrm{ppm}$ (parts per million) for ${ }^{125} \mathrm{Te}$, solid $\mathrm{NaCl}$ at $0 \mathrm{ppm}$ for ${ }^{35} \mathrm{Cl}$, and $0.5 \mathrm{M}$ $\mathrm{Pb}\left(\mathrm{NO}_{3}\right)_{2 \text { (aq) }}$ at $-2941 \mathrm{ppm}$ where $\mathrm{Pb}\left(\mathrm{CH}_{3}\right)_{4}$ is 0 ppm for ${ }^{207} \mathrm{~Pb}$, respectively.

Analysis of the complete ${ }^{125} \mathrm{Te}$ and ${ }^{207} \mathrm{~Pb}$ lineshapes was accomplished with the SIMPSON software package (Bak et al., 2000) as well as other software developed at the Environmental Molecular Sciences Laboratory (EMSL, http://www.emsl.pnl.gov/emslweb/). Simulations of the NMR spectra were performed on a Beowulf cluster at the Pacific Northwest National Laboratory. The fitting procedure utilized for the experimental lineshape was a standard SIMPLEX algorithm within the SIMPSON program. Optimizations of the complete NMR lineshapes were performed with ideal radio frequency pulses, stepped offsets were not simulated in this case. We define the shielding anisotropy as $\Delta \sigma=\delta_{33}-$ $1 / 2\left(\delta_{11}+\delta_{22}\right)$, where the elements of the shielding tensor are ordered as $\left|\delta_{33}-\delta_{\text {iso }}\right| \geq\left|\delta_{11}-\delta_{\text {iso }}\right| \geq\left|\delta_{22}-\delta_{\text {iso }}\right|$. Incorporating a Gaussian disorder to the isotropic chemical shift on the order of $100 \mathrm{ppm}$ mimicked the amorphous nature of the material. Simulations were calculated between $\pm 2 \sigma(200 \mathrm{ppm})$ in $20 \mathrm{ppm}$ steps and scaled appropriately before summing into the final lineshape. 


\section{RESULTS \& DISCUSSION}

\subsection{Halide Mineral Processing in FY2011}

\subsubsection{X-Ray Diffraction}

The sodalite pellets that we made in FY2010 without sintering aids showed moderate sodalite concentrations of $\sim 60$ mass $\%$, mostly independent of the firing temperature (Figure 4.1). However, we were able to increase these values with some modifications to the process that included the addition of NBS-1, a glass sintering aid, and alternative Group IV component substitutions for the CS.
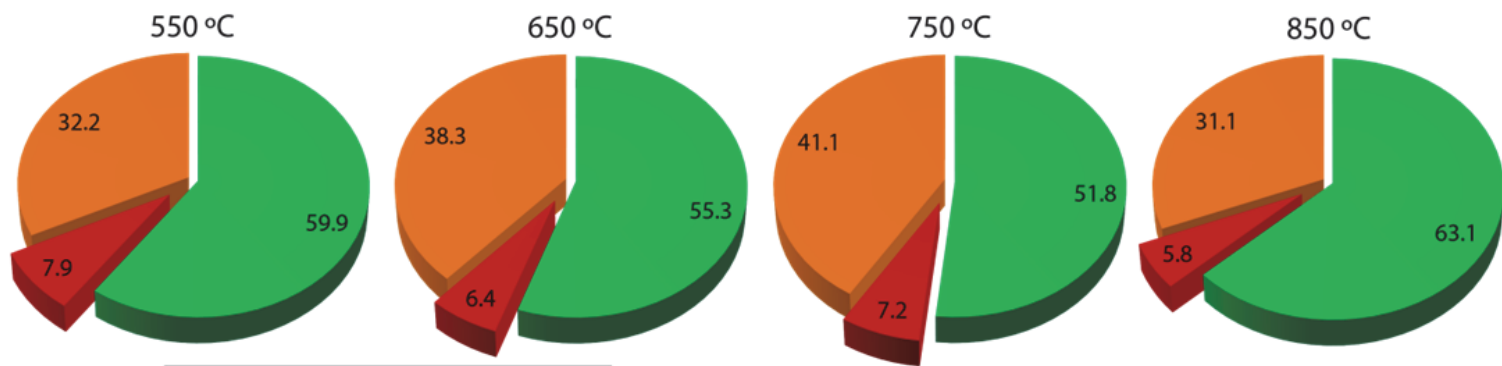

\section{Sodalite | $\square$ Zeolite | $\square$ Salt}

Figure 4.1. Summary of phase distribution in S5A specimens at different firing temperatures (mass \%).

Figure 4.2 provides a comparison between the as-made, unfired, and dried gel of the S5A series specimens made with CS in FY2010, S5C made with TEOS in FY2011, and S5D made with Ge-EtO in FY2011. Here, it is obvious that these slight modifications to the process resulted in widely different phase distributions. The unfired S5A specimen had a very small fraction of sodalite at $24 \%$ but was increased to 76 mass $\%$ in a separate specimen that was dried at $90{ }^{\circ} \mathrm{C}$ for 5 days. The $\mathrm{S} 5 \mathrm{C}$ specimen had a very large sodalite fraction $(96 \%)$ with the S5D specimen not much lower (82\%). This suggests that an organic form of the Group IV component may be the preferred precursor to achieve a large sodalite fraction in the as-made gel. Also, with the organic precursors, the fraction of unreacted salt was reduced.

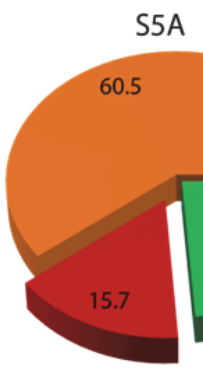

$\mathrm{S} 5 \mathrm{~A}$

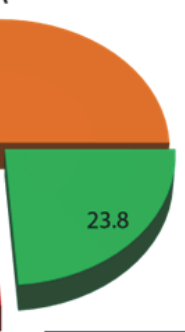

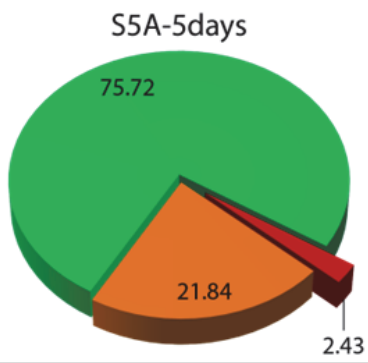
2.43

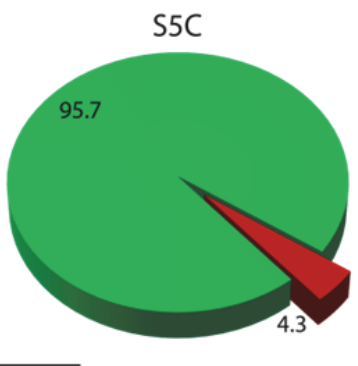

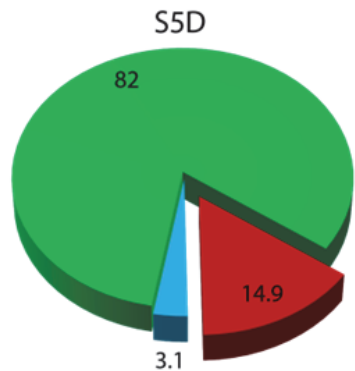

\section{Sodalite | $\square$ Zeolite | $\square$ Salt | $\square$ Others}

Figure 4.2. Summary of phase distribution in the S5A, S5A after drying for 5-days at $90{ }^{\circ} \mathrm{C}, \mathrm{S} 5 \mathrm{C}$, and S5D formulations as-made (unfired) (mass \%).

Figure 4.3 provides a comparison summary of the phase distribution in pellets made with different sintering aids and fired at different temperatures. All of the SA-\#\#\# sintering aids behaved similarly with a maximum sodalite concentration at the lower sintering temperatures $\left(550-600^{\circ} \mathrm{C}\right)$ and an inverse correlation between the sodalite and $\mathrm{Na}-\mathrm{Al}-\mathrm{Si}-\mathrm{O}$ phase concentrations with increasing firing temperatures. This is likely due to chloride volatility with increased firing temperatures. This inverse relationship was similarly observed with S5C-TePbO and S5C-NBS1 but was highly exaggerated with S5C-NaAlP. 

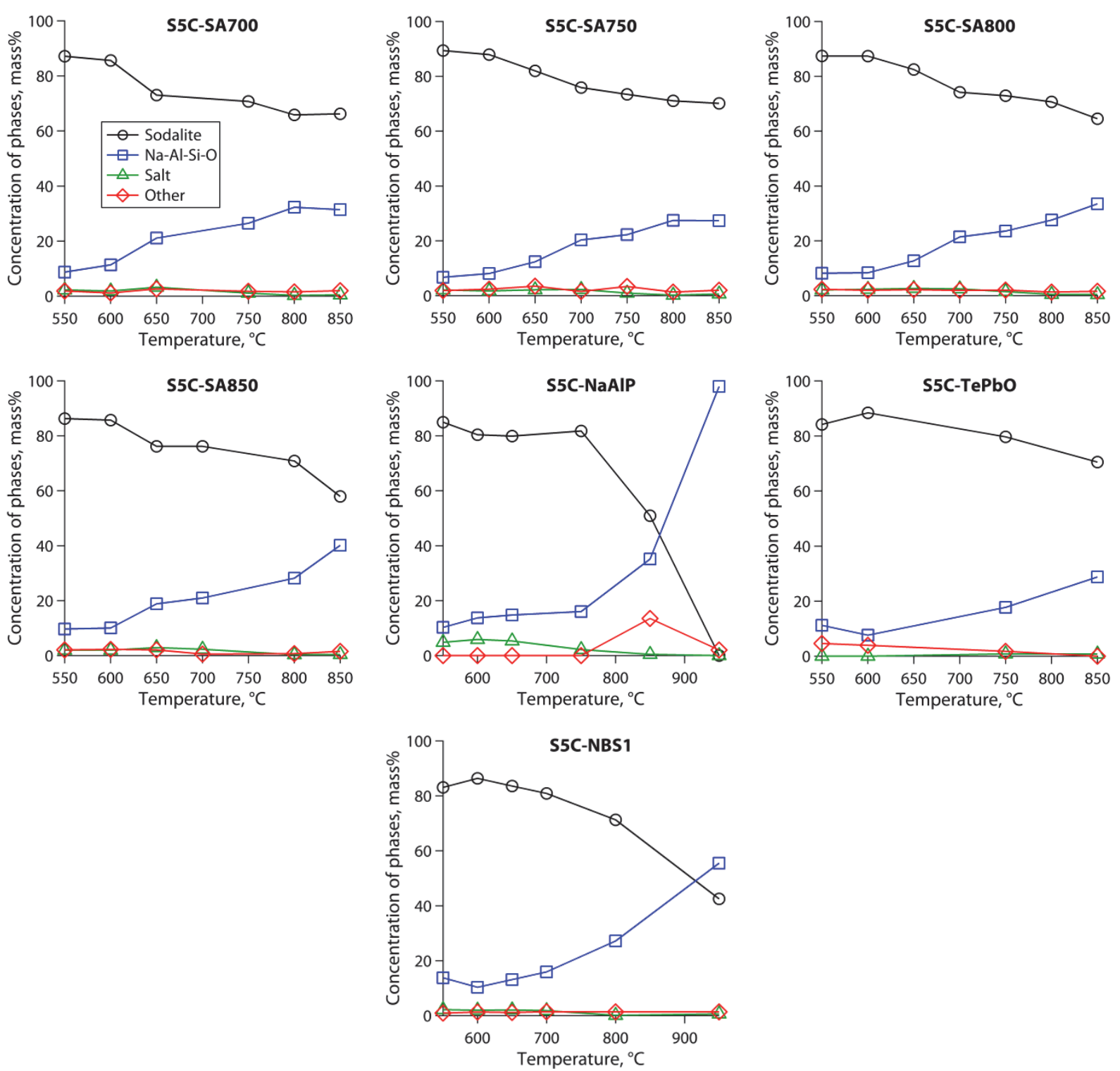

Figure 4.3. Phase distribution for the various S5C formulations at fired at different temperatures. The legend presented in the upper left figure is valid for all figure parts.

All of the crystalline phases used for the Rietveld refinement of the sodalite specimens are summarized in Table 4.1. These phases are similar to those found with the CS-based sodalites we made in FY2010, although in some cases, alternative phases were found for the sodalite and Na-Al-Si-O structural fits. More information on the exact phases found for each specimen and the actual Rietveld refinement patterns were presented in our earlier work (Riley et al., 2011). 
Table 4.1. Summary of phases identified in the halide minerals. The table shows powder diffraction file (PDF) and International Crystal Structure Database (ICSD) identifications, the space group (SG), the space group number (SG\#), and references for the patterns. More details can be found in our earlier work (Riley et al., 2011).

\begin{tabular}{llllll}
\hline Phase ID & Category & PDF\# & ICSD\# & SG (SG\#) & Reference \\
\hline Cancrinite & Cancrinite & $83-1183$ & 80298 & $P 3(143)$ & (Lindner et al., 1995) \\
\hline $\mathrm{Na}_{8}\left(\mathrm{AlSiO}_{4}\right)_{6} \mathrm{Cl}_{2}$ & Sodalite & $37-0476$ & 41188 & $P \overline{4} 3 n(218)$ & (Beagley et al., 1982) \\
Hydrosodalite & Sodalite & $75-5343$ & 413502 & $P \overline{4} 3 n(218)$ & (Kendrick and Dann, 2004) \\
$\mathrm{Na}_{8}\left(\mathrm{AlGeO}_{4}\right)_{6} \mathrm{Cl}_{2}$ & Sodalite & $79-0711$ & 65664 & $P \overline{4} 3 n(218)$ & (Fleet, 1989) \\
\hline Carnegieite low & NaAlSiO & $44-1496$ & 73511 & $P b 21 a(29)$ & (Withers and Thompson, 1993) \\
Nepheline & NaAlSiO & $76-2465$ & 37354 & $P 63(173)$ & (Foreman and Peacor, 1970) \\
$\mathrm{Na}_{1.55} \mathrm{Al}_{1.55} \mathrm{Si}_{0.45} \mathrm{O}_{4}$ & NaAlSiO & $49-0006$ & 280473 & $P b c a(61)$ & (Thompson et al., 1998a) \\
$\mathrm{Analcime} 1 \mathrm{Q}$ & NaAlSiO & $73-6448$ & 34058 & $I 41 / A C D(142)$ & (Meier, 1973) \\
$\mathrm{Sylvite}(\mathrm{KCl})_{\mathrm{LiCl}}$ & Salt & $41-1476$ & 28938 & $F m 3 m(225)$ & (Barrett and Wallace, 1954) \\
$\mathrm{Halite}(\mathrm{NaCl})_{\mathrm{Li}_{2} \mathrm{SiO}_{3}}$ & Salt & $74-1972$ & 27981 & $F m 3 m(225)$ & (Ott, 1923) \\
$\mathrm{Y}_{6} \mathrm{Cl}_{10}\left(\mathrm{Si}_{4} \mathrm{O}_{12}\right)$ & Other & $05-0628$ & 28948 & $F m 3 m(225)$ & (Barrett and Wallace, 1954) \\
\hline
\end{tabular}

The sodalite fraction in $\mathrm{S} 5 \mathrm{D}$, the as-made germania-based sample, was high at 82 mass $\%$ as shown in Figure 4.4. The as-made material was pressed and fired at $750{ }^{\circ} \mathrm{C}$ and the open porosity was high $(>40 \%)$ so this option was abandoned without further investigation. However, this approach of including germania-based precursors or sintering aids could be considered in the future since the results were far superior to thermal methods.

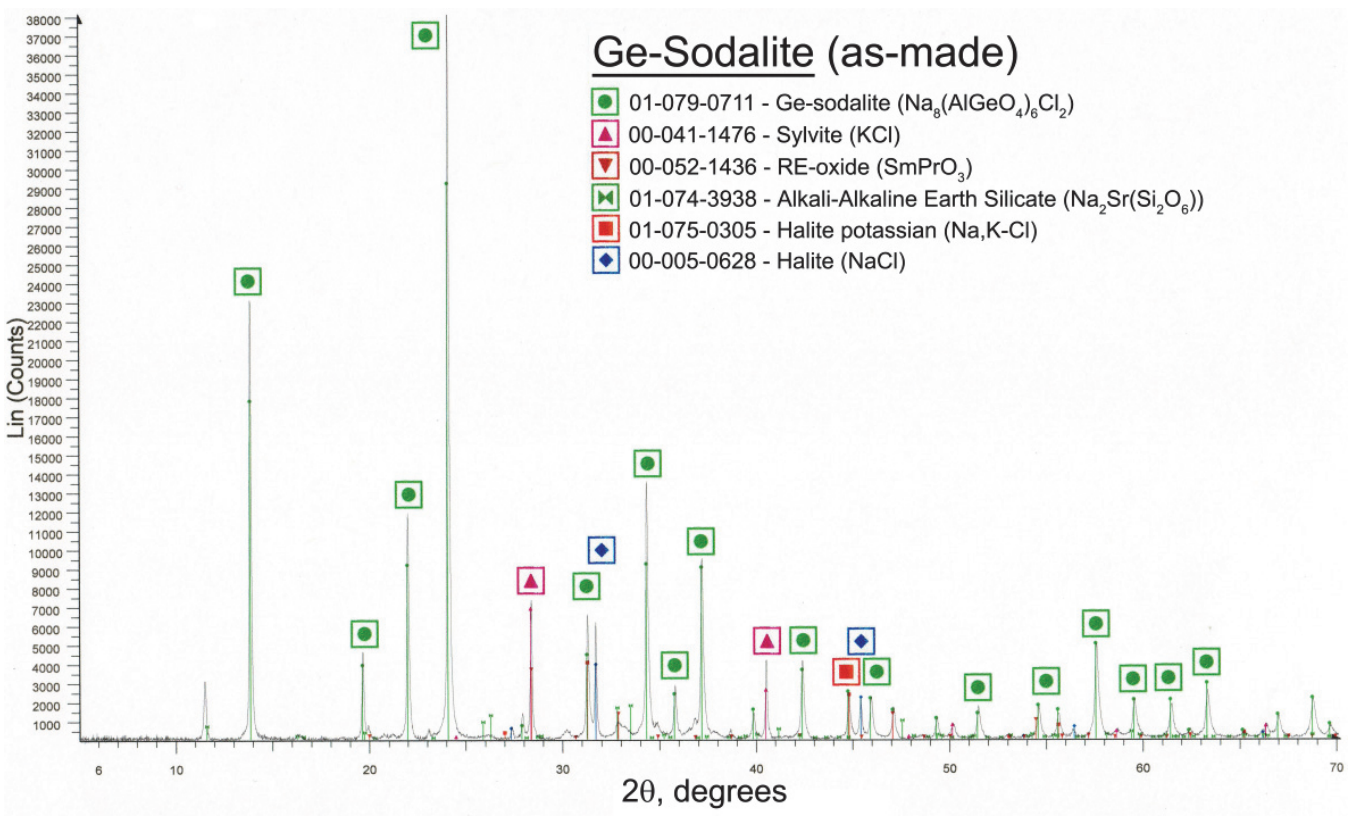

Figure 4.4. Summary of phase analysis for the as-made S5D material. 


\subsubsection{Pellet Densities and Porosity}

The data set for the alternative sintering aids is presented in Table 4.2, with a partial graphical representation in Figure 4.5. It should be noted that the S5C-NBS1 sample is a S5C sample made in FY2011 with TEOS. Here, we see that the maximum \% of $\rho_{\mathrm{t}}$ with the NBS-1 glass sintering aid was observed around $950{ }^{\circ} \mathrm{C}$ at $87 \%$, although it had not plateaued at that point [see Equation (8)]. With the new sintering aids, densities of up to $92 \%$ of $\rho_{\mathrm{t}}$ were achieved. The optimum heat-treatment temperature to achieve maximum density was $750{ }^{\circ} \mathrm{C}$ for SA-700, SA-800, and SA-850 while it was $850{ }^{\circ} \mathrm{C}$ for SA-750 (Figure 4.5a). The NaAlP-B4 and TePbO and sintering aids did not help densify the pellets; even at $850{ }^{\circ} \mathrm{C}$ firing temperatures the densities were still $<60 \%$ of $\rho_{\mathrm{t}}$. This suggests that these sintering aid compositions were not as reactive with the mineral components as the SA-\#\#\# and NBS-1 sintering aids.

Figure $4.5 \mathrm{~b}$ provides a comparison of the density and sodalite mass $\%$ for each sintering aid at the $750{ }^{\circ} \mathrm{C}$ firing temperature. From this plot, it is evident that the SA-\#\#\# sintering aids were effective at closing the pores and allowing a good amount of sodalite to form during firing. However, at this temperature, the SA-\#\#\# sintering aids are not much more efficient than the NBS-1 glass. Also, the effect of increased viscosity for NBS-1 over the SA-\#\#\# sintering aids is evident by the high porosity for S5C-NBS1 at temperatures $\leq 750{ }^{\circ} \mathrm{C}$.
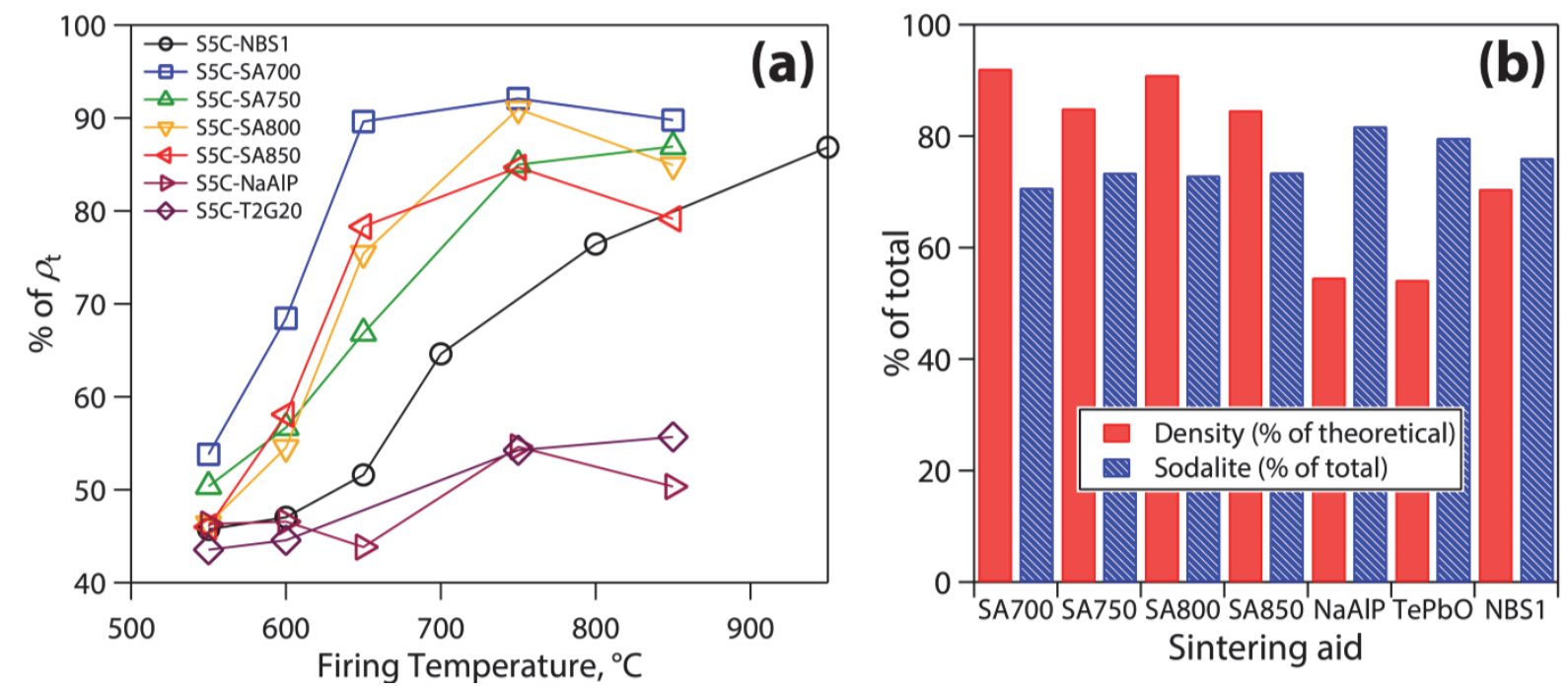

Figure 4.5. (a) Summary of $\%$ of $\rho_{\mathrm{t}}$ as a function of firing temperature for S5C series specimens made with an additional 5 mass $\%$ of various sintering aids (see Table 3.2 for more details). (b) Column chart comparing the mass $\%$ of sodalite in each specimen with the $\%$ of $\rho_{\mathrm{t}}$ for all $\mathrm{S} 5 \mathrm{C}$ specimens fired at $750{ }^{\circ} \mathrm{C}$. 
Table 4.2. Summary of sodalite mass $\%, \%$ porosity, calculated $\rho_{\mathrm{t}}$, and $\%$ of $\rho_{\mathrm{t}}$ for specimens containing 5 mass $\%$ additions of various sintering aids and fired at different temperatures. The maxima for sodalite and $\%$ of $\rho_{\mathrm{t}}$ are shown in bold type.

\begin{tabular}{|c|c|c|c|c|c|}
\hline Specimen ID & Firing Temperature, ${ }^{\circ} \mathrm{C}$ & Sodalite (mass\%) & $\%$ Porosity & $\rho_{\mathrm{t}}$ & $\%$ of $\rho_{\mathrm{t}}$ \\
\hline \multirow[t]{6}{*}{ S5C-NBS1 } & 550 & 83.1 & $56 \%$ & 2.32 & $46 \%$ \\
\hline & 600 & 86.4 & $55 \%$ & 2.32 & $47 \%$ \\
\hline & 650 & 83.6 & $51 \%$ & 2.32 & $52 \%$ \\
\hline & 700 & 80.9 & $39 \%$ & 2.33 & $65 \%$ \\
\hline & 800 & 71.3 & $26 \%$ & 2.38 & $76 \%$ \\
\hline & 950 & 42.5 & $15 \%$ & 2.43 & $87 \%$ \\
\hline \multirow[t]{5}{*}{ S5C-SA700 } & 550 & 87.2 & $49 \%$ & 2.32 & $54 \%$ \\
\hline & 600 & 85.6 & $34 \%$ & 2.31 & $68 \%$ \\
\hline & 650 & 73.0 & $13 \%$ & 2.35 & $90 \%$ \\
\hline & 750 & 70.7 & $11 \%$ & 2.37 & $92 \%$ \\
\hline & 850 & 66.2 & $14 \%$ & 2.37 & $90 \%$ \\
\hline \multirow[t]{7}{*}{ S5C-SA750 } & 550 & 89.4 & $51 \%$ & 2.31 & $50 \%$ \\
\hline & 600 & 87.9 & $45 \%$ & 2.32 & $57 \%$ \\
\hline & 650 & 82.0 & $35 \%$ & 2.34 & $67 \%$ \\
\hline & 700 & 75.9 & $28 \%$ & 2.34 & $74 \%$ \\
\hline & 750 & 73.4 & $18 \%$ & 2.38 & $85 \%$ \\
\hline & 800 & 71.1 & $20 \%$ & 2.36 & $84 \%$ \\
\hline & 850 & 70.1 & $16 \%$ & 2.37 & $87 \%$ \\
\hline \multirow[t]{7}{*}{ S5C-SA800 } & 550 & 87.5 & $56 \%$ & 2.32 & $46 \%$ \\
\hline & 600 & 87.4 & $48 \%$ & 2.32 & $55 \%$ \\
\hline & 650 & 82.5 & $27 \%$ & 2.33 & $75 \%$ \\
\hline & 700 & 74.2 & $15 \%$ & 2.35 & $87 \%$ \\
\hline & 750 & 72.9 & $12 \%$ & 2.36 & $91 \%$ \\
\hline & 800 & 70.7 & $15 \%$ & 2.36 & $89 \%$ \\
\hline & 850 & 64.5 & $18 \%$ & 2.39 & $85 \%$ \\
\hline \multirow[t]{6}{*}{ S5C-SA850 } & 550 & 86.3 & $56 \%$ & 2.32 & $46 \%$ \\
\hline & 600 & 85.7 & $44 \%$ & 2.32 & $58 \%$ \\
\hline & 650 & 76.2 & $24 \%$ & 2.34 & $78 \%$ \\
\hline & 700 & 76.2 & $19 \%$ & 2.33 & $83 \%$ \\
\hline & 800 & 70.8 & $18 \%$ & 2.36 & $86 \%$ \\
\hline & 850 & 57.9 & $23 \%$ & 2.41 & $79 \%$ \\
\hline \multirow[t]{4}{*}{$\mathrm{S} 5 \mathrm{C}-\mathrm{TePbO}$} & 550 & 84.2 & $59 \%$ & 2.33 & $44 \%$ \\
\hline & 600 & 88.4 & $58 \%$ & 2.32 & $45 \%$ \\
\hline & 750 & 79.7 & $52 \%$ & 2.27 & $54 \%$ \\
\hline & 850 & 70.5 & $51 \%$ & 2.33 & $56 \%$ \\
\hline \multirow[t]{6}{*}{ S5C-NaAlP } & 550 & 84.9 & $55 \%$ & 2.29 & $46 \%$ \\
\hline & 600 & 80.4 & $55 \%$ & 2.30 & $47 \%$ \\
\hline & 650 & 79.7 & $58 \%$ & 2.29 & $44 \%$ \\
\hline & 750 & 81.8 & $48 \%$ & 2.30 & $55 \%$ \\
\hline & 850 & 50.9 & $50 \%$ & 2.48 & $50 \%$ \\
\hline & 950 & 0.0 & $54 \%$ & 2.54 & $50 \%$ \\
\hline
\end{tabular}


An effective way of comparing the data was to graph the $\%$ of $\rho_{\mathrm{t}}$ and the sodalite (mass $\%$ ) on the same plot, since the goal primary goal of this work was to maximize both of these parameters. Figure 4.6 provides a comparison of density versus sodalite where the most promising candidates are in the upper right corner of the plot (S6 specimens).

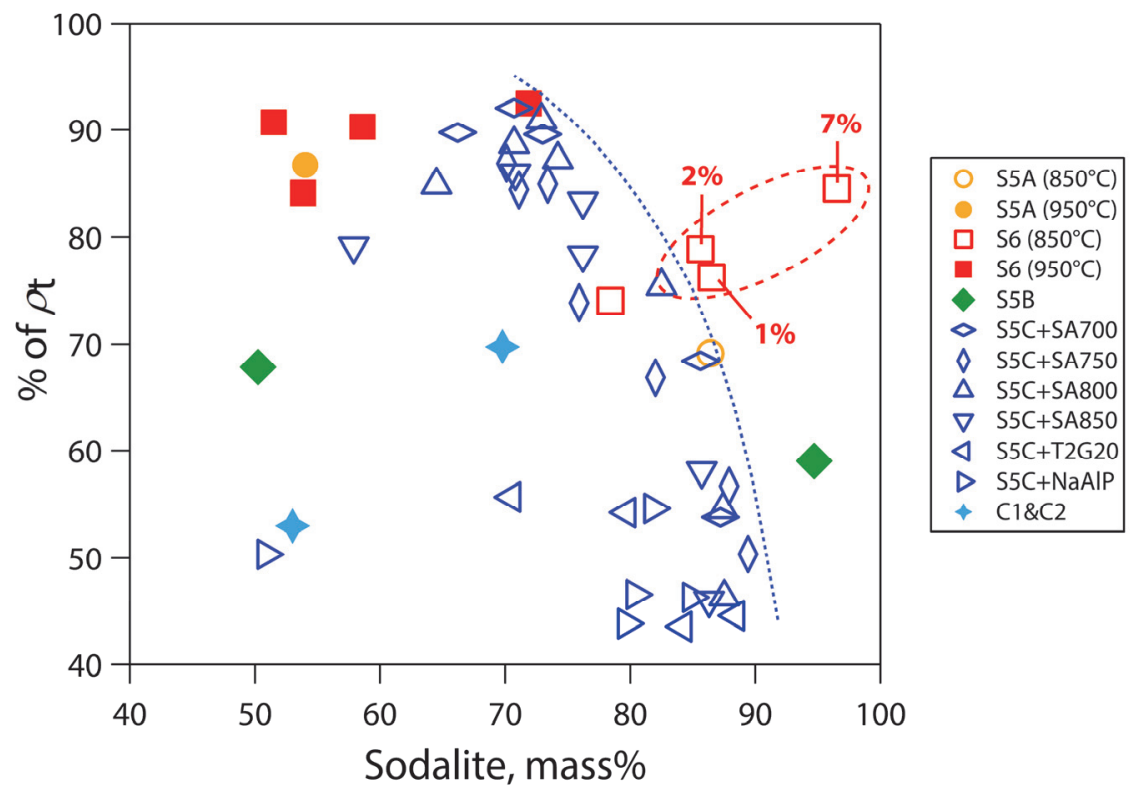

Figure 4.6. Comparison of $\rho_{\mathrm{t}}$ and mass\% sodalite for the $\mathrm{S} 5 \mathrm{~A}, \mathrm{~S} 5 \mathrm{~B}, \mathrm{~S} 5 \mathrm{C}, \mathrm{S} 6$, and $\mathrm{C} 1 / \mathrm{C} 2$ series specimens (at mixed firing temperatures). The dotted blue line outlines the maxima boundary for TEOSbased pellets.

\subsubsection{Scanning electron microscopy}

Backscattered SEM micrographs and EDS dot mapping provided high contrast visual comparisons of the phase distribution in these specimens. A clear distinction was found between the sodalite (with chloride) and nepheline phases in the $\mathrm{S} 6-7 \% 750^{\circ} \mathrm{C}$ (Figure 4.7) and $\mathrm{S} 5 \mathrm{C}-850^{\circ} \mathrm{C}$ (Figure 4.8) pellets. Figure 4.7 clearly shows the Ln separation from the bulk material as well as a distinct difference in the $\mathrm{Si}, \mathrm{K}$, and $\mathrm{Al}$ content between the nepheline and sodalite phases. Figure 4.8 provides some SEM comparisons between solution-based materials made with colloidal silica and TEOS as the silica source where the TEOS-based product has $\sim 10$ times smaller grain size. Additionally, Figure 4.8 shows something similar to Figure 4.7 where the nepheline and sodalite are separate phases. Also, the particle sizes of $\mathrm{S} 5 \mathrm{C}-850^{\circ} \mathrm{C}$ are much smaller than those in $\mathrm{S} 6-7 \% 750^{\circ} \mathrm{C}$. However, for the most part, $\mathrm{S} 6-7 \% 750^{\circ} \mathrm{C}$ (colloidal silica) and S5C$850^{\circ} \mathrm{C}$ (TEOS) pellets are more similar than expected based on fabrication methods and the chemical reactants used. The presence of free lanthanides is denoted by the La map.
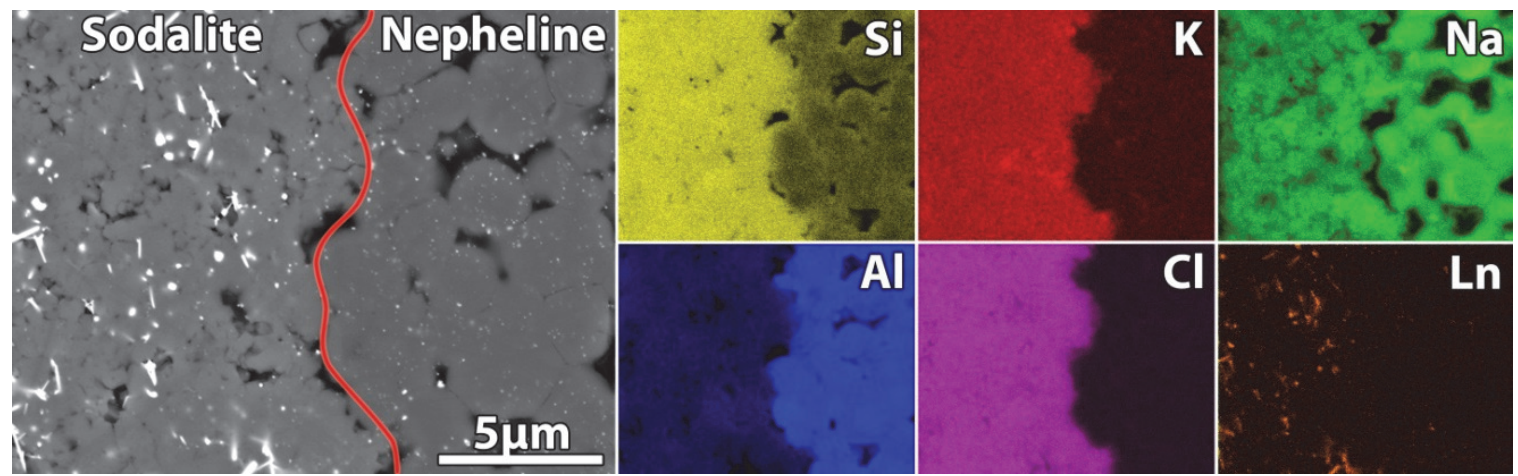

Figure 4.7. SEM micrograph and EDS dot map of S6 pellet with $7 \%$ NBS-1 glass $\left(\mathrm{S} 6-7 \% 750^{\circ} \mathrm{C}\right)$. 


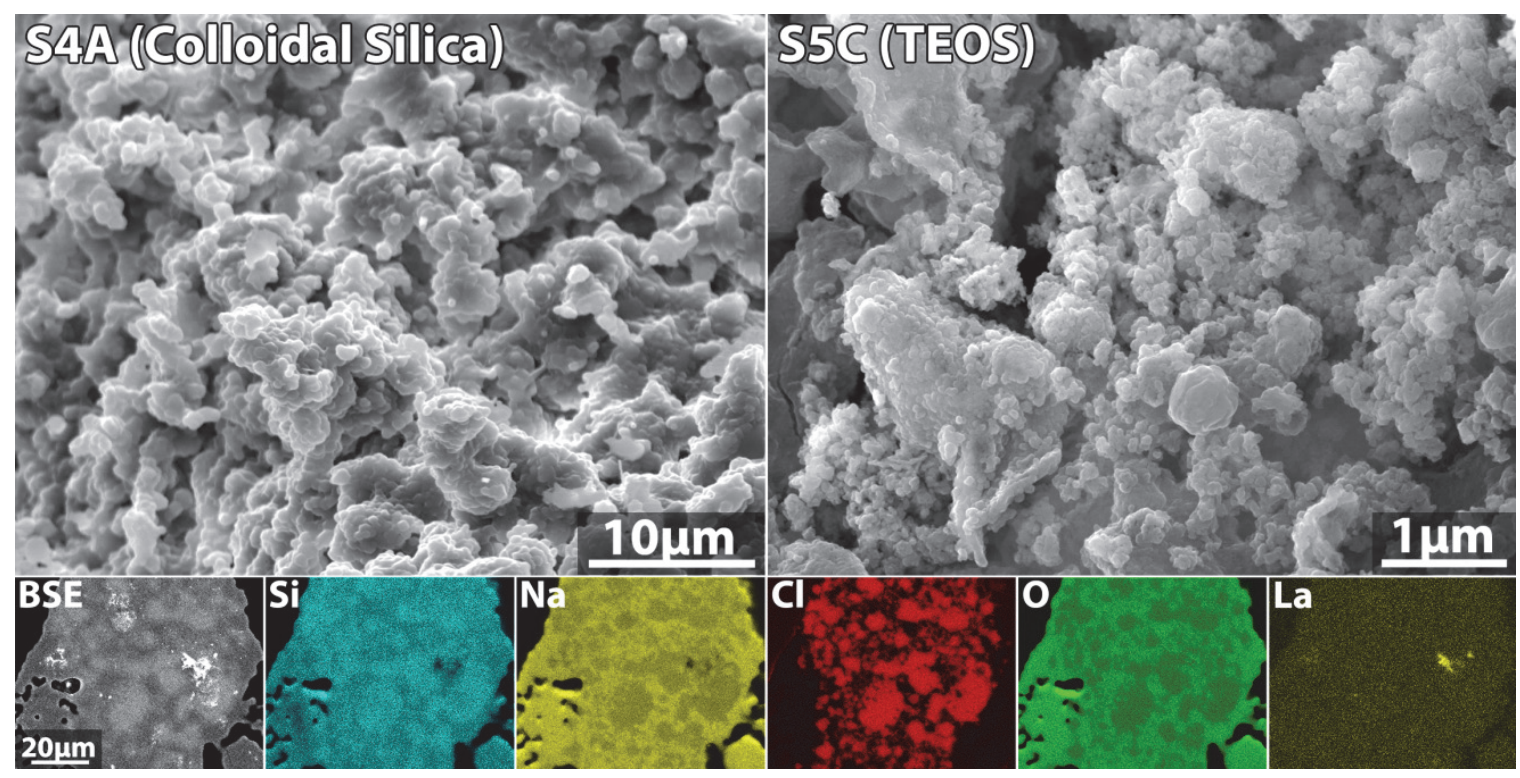

Figure 4.8. SEM micrographs of S4A, S5C, and a dot map on S5C (TEOS-based) showing elemental distribution and a clear distinction between the chloride-containing phase (sodalite), the phase without chloride (nepheline), and the lanthanide phase (shown as La).

\subsubsection{Thermal Properties}

The thermal diffusivity of the Sodalite 5A pellets was quantified with the NETZSCH LFA 457 using pellets (see Table 2.4 for the composition). The results from these measurements are presented in Figure 4.9. These results show that the thermal diffusivity of the S5A specimens ranged from $0.17-0.40 \times 10^{-6} \mathrm{~m}^{2} / \mathrm{s}$ with a maximum value at the lower temperature and a steady decrease with increasing temperature.

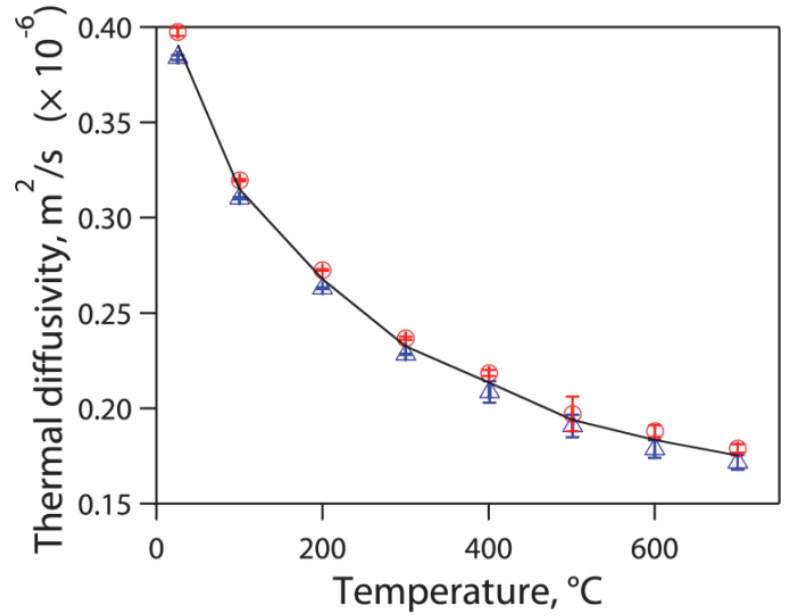

Figure 4.9. Thermal diffusivity of Sodalite $5 \mathrm{~A}$ pellets. Error bars for the measured thermal diffusivity are included on the data points. 


\subsection{Halide Mineral Processing in FY2012}

The $\mathrm{pH}$ values of the different solutions are presented in Table 4.3. The $\mathrm{pH}$ of the salt is slightly acidic (4.78) and the addition of the colloidal silica raises is to near-normal $\mathrm{pH}(\sim 6.6)$. The addition of the $\mathrm{NaOH}$ or $\mathrm{NaAlO}_{2}$ brings the $\mathrm{pH}$ to a very basic solution $(\sim 13)$. Even though the contents of these solutions were different, the final $\mathrm{pH}$ ranges were surprisingly similar (12.99-13.10).

Table 4.3. Summary of solution $\mathrm{pH}$ results during sample preparation. Each value is listed as an additive value where the solution contains all of the contents listed above that additive.

\begin{tabular}{l|c|c|c}
\hline Solution $\mathrm{pH}$ after component addition & SM-1 & SM-2 & SM-3 \\
\hline Salt $\left(X \mathrm{XCl}_{20}\right)$ & 4.78 & 4.78 & - \\
Colloidal silica & 6.60 & 6.56 & - \\
$\mathrm{NaOH}+\mathrm{Al}(\mathrm{OH})_{3}$ & 12.99 & - & - \\
$\mathrm{NaAlO}_{2}$ powder $+75 \mathrm{~mL} \mathrm{DIW}$ & - & 13.02 & - \\
$\mathrm{Metakaolin}^{+} \mathrm{NaOH}$ & - & - & 13.14 \\
Metakaolin $+\mathrm{NaOH}+$ Salt & - & - & 13.10 \\
\hline
\end{tabular}

The dried powders showed low sodalite production and some undissolved salts, even after calcination for $2-4 \mathrm{~h}$ at $600{ }^{\circ} \mathrm{C}$. However, when fired at $650{ }^{\circ} \mathrm{C}$ for $8 \mathrm{~h}$, some specimens showed a high sodalite fraction suggesting that the limiting factors to producing sodalite at lower temperatures might be heat-treatment time and the close contact of the powders that is achieved when they are pressed together before firing. Some of the SM-2 formulations actually showed up to 100 mass $\%$ sodalite at the $650{ }^{\circ} \mathrm{C}$ firing temperature (but with reduced densities), e.g., SM1-10\%-C- $650^{\circ} \mathrm{C}\left(92 \%\right.$ sodalite), SM2-C-5\%-650 ${ }^{\circ} \mathrm{C}$ ( $100 \%$ sodalite), and SM3-AM- $5 \%-650^{\circ} \mathrm{C}$ (96\% sodalite). After considering all of the options, the most effective results were obtained with the sintering aid additions. For the 5\% SA800 specimens, the best all-around conditions were the SM\#-5\%-C specimens.

A summary of all the data points collected is presented in Figure 4.10. The values used for density are an average of the measurements obtained with the two different methods and the $\rho_{\mathrm{t}}$ was calculated with Equation (8), taking into account the individual densities of all the phases present in the samples. The density of chloride-based sodalites varies by composition at $2.27-2.33 \times 10^{3} \mathrm{~kg} / \mathrm{m}^{3}$ (Barth, 1932b; Cressey, 2004; Frank et al., 2005; McMullan et al., 1996) and the density of the alkali aluminosilicates are elevated when compared to sodalite at $\sim 2.54-2.67 \times 10^{3} \mathrm{~kg} / \mathrm{m}^{3}$, depending on the composition of the phase composition (Dollase and Thomas, 1978b; Thompson et al., 1998b). These calculations do not consider the increased density of the SA800 sintering aid $\left(2.55 \times 10^{3} \mathrm{~kg} / \mathrm{m}^{3}\right)$.

For the SM-1 specimens, $75 \%$ had a sodalite fraction exceeding 75 mass $\%,>50 \%$ had a sodalite fraction $\geq 87$ mass $\%$, and only one showed $100 \%$ sodalite (SM1-10\%-C- $950^{\circ} \mathrm{C}$ ). For the SM-2 specimens, $75 \%$ had $>90$ mass $\%$ sodalite regardless of the processing method while $45 \%$ had 100 mass $\%$ sodalite. The SM-3 specimens produced the widest range of sodalite content of 2.8-96 mass\%. These variations are attributed to the different reactivities and starting particle sizes of the precursors used in each formulation.

In general, the calcination process seemed to improve overall pellet density and sodalite production. This is likely due to the fact that calcination removes any physisorbed or chemisorbed water from the powder. If this water is not removed before the pellets are pressed, it will evolve during the firing process and result in voids within the pellet. This is supported by increased mass losses after firing the pellets for uncalcined specimens. The most satisfactory results were obtained with the calcined specimens that contained the sintering aid, e.g., SM\#-5\%-C. The XRD results for each specimen processed under these particular conditions at the most effective firing temperatures showed a high sodalite fraction (Figure 4.11). Minor phases found for $\mathrm{SM} 3-5 \%-\mathrm{C}-750^{\circ} \mathrm{C}$ were $\mathrm{KAlO}_{2}$ (Sokolowski and Kotarba, 2000) and $\mathrm{K}_{1.6} \mathrm{Na}_{6} \mathrm{Al}_{7.49} \mathrm{Si}_{8.51} \mathrm{O}_{32}$ (nepheline) (Tait et al., 2003a); $\mathrm{NaCl}$ was also found in $\mathrm{SM}-1$ (not shown). 


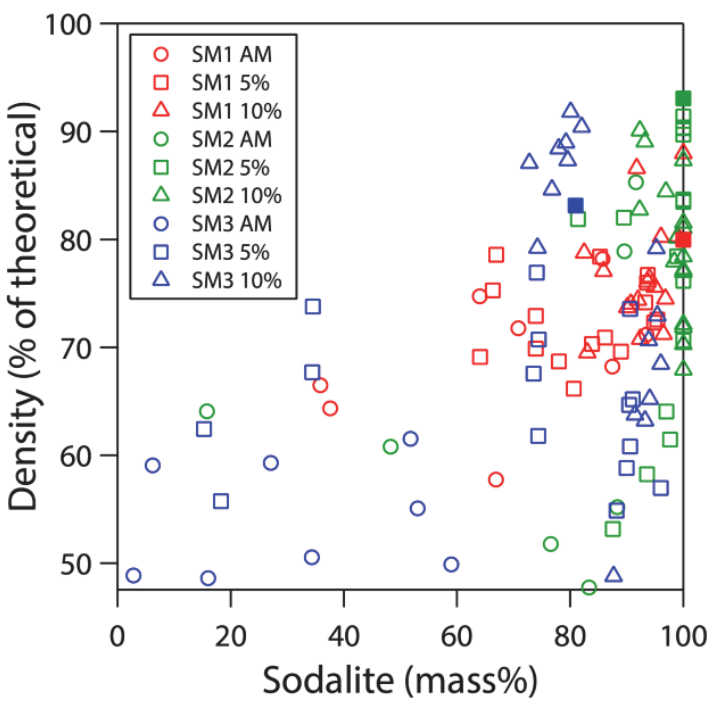

Figure 4.10. Summary of density ( $\%$ of theoretical) as a function of sodalite concentration (mass $\%$ ) for all data. The filled squares are the PVS specimens with 5\% SA800 that were isostatically pressed at $3.4 \times 10^{8} \mathrm{~Pa}\left(5.0 \times 10^{4} \mathrm{PSI}\right)$.

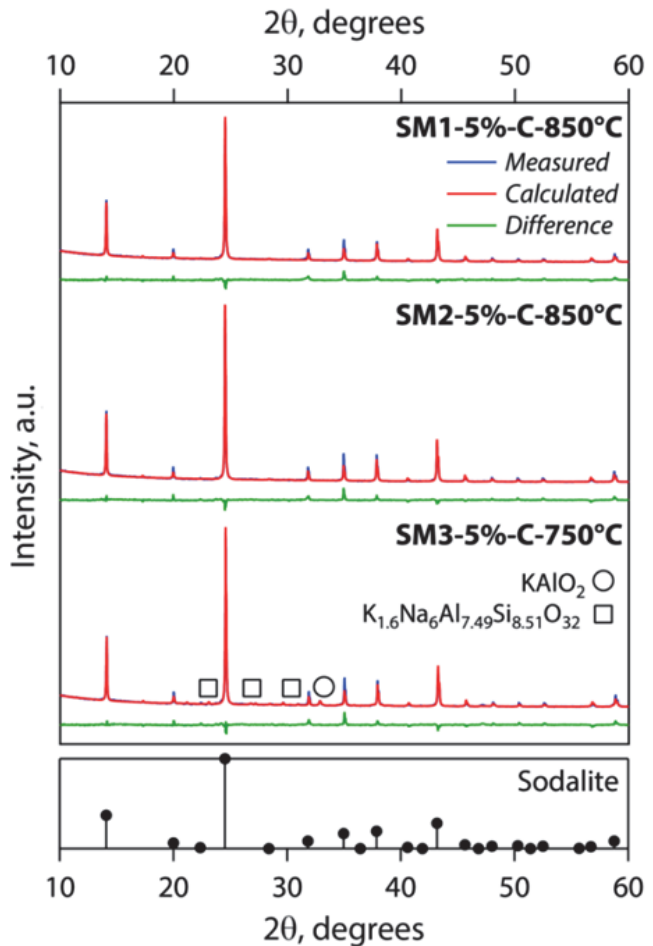

Figure 4.11. XRD summary figure showing sodalite (space group $=P \overline{4} 3 n$ ) as the primary phase for these three specimens SM1-5\%-C- $850^{\circ} \mathrm{C}$, SM2-5\%-C- $850^{\circ} \mathrm{C}$, and SM3-5\%-C-750 ${ }^{\circ} \mathrm{C}$ with a unit cell of $a=$ $0.882 \mathrm{~nm}, 0.889 \mathrm{~nm}$, and $0.887 \mathrm{~nm}$, respectively (Barth, 1932a). The minor phases were $\mathrm{KAlO}_{2}$ (Sokolowski and Kotarba, 2000) and $\mathrm{K}_{1.6} \mathrm{Na}_{6} \mathrm{Al}_{7.49} \mathrm{Si}_{8.51} \mathrm{O}_{32}$ (nepheline) (Tait et al., 2003a) for $\mathrm{SM} 3-5 \%-\mathrm{C}-750^{\circ} \mathrm{C}$. This plot shows the raw measured data (blue), fitted/calculated spectra (red), and difference patterns (green) for each spectra. 


\subsubsection{SM-1 (Gibbsite) Overview}

Without a sintering aid, the SM-1 pellets with the lowest porosity were found at the lower firing temperatures. The mass $\%$ of sodalite in the SM1-AM- $850^{\circ} \mathrm{C}$ and $\mathrm{SM} 1-\mathrm{AM}-\mathrm{C}-850^{\circ} \mathrm{C}$ specimens were high at $94 \%$ and $88 \%$ but with reduced densities at $71 \%$ and $68 \%$ of $\rho_{\mathrm{t}}$, respectively. When 5 or 10 mass $\%$ SA 800 was added, firing at $950{ }^{\circ} \mathrm{C}$ produced the highest densities $\left(74-88 \%\right.$ of $\left.\rho_{\mathrm{t}}\right)$. The addition of 10 mass $\%$ SA800 improved all SM-1 specimens with noticeably higher sodalite fractions and densities. The best specimen for the SM-1 method was SM1-10\%-C at $950{ }^{\circ} \mathrm{C}$ at 100 mass $\%$ sodalite and $88 \%$ of $\rho_{\mathrm{t}}$. For these pellets, higher firing temperatures such as $850-950{ }^{\circ} \mathrm{C}$ and $5-10$ mass $\%$ of this sintering aid would be required to achieve high sodalite and low porosity in pellets made with this synthesis method.

\subsubsection{SM-2 $\left(\mathrm{NaAlO}_{2}\right)$ Overview}

The SM-2 formulations proved the most effective at forming sodalite and were a noticeable improvement over our previous work with increased densification at lower temperatures and very high sodalite fractions. This is likely because this method provided the most effective mixing of the precursors; the $\mathrm{NaAlO}_{2}$ was completely dissolved in solution when it was added to the mixture during the synthesis process.

Without a sintering aid, the SM-2 specimens showed the highest sodalite at the $750{ }^{\circ} \mathrm{C}$ firing temperature (SM2-AM- $750^{\circ} \mathrm{C}, 83 \%$ ), although the density was very low at $48 \%$ of $\rho_{\mathrm{t}}$. The SM2-AM-C specimens showed a consistent increase in sodalite between 750 and $950{ }^{\circ} \mathrm{C}$ at $88-92$ mass\% with a steady increase in density from $55 \%$ to $85 \%$ of $\rho_{\mathrm{t}}$, respectively. With the addition of 5 mass $\%$ SA800, specimens fired at $850{ }^{\circ} \mathrm{C}$ showed the highest sodalite fractions.

The best specimen for the SM-2 formulation was SM2-5\%-C- $850^{\circ} \mathrm{C}$ at $100 \%$ sodalite and $91 \%$ of $\rho_{\mathrm{t}}$. Further analysis of this specimen with SEM-EDS showed that two distinct compositions were present (Figure 4.12). Cluster phase analysis on this map with the Genesis software showed two distinctly different sodalite phases with similar, yet not identical compositions. The Na:K ratios were identified (80.8:1 for red and 33.6:1 for blue) while analyses on the rest of the components were shown to be congruent (Figure 4.13). This indicates that the chemical segregation identified could have a few different causes including: (1) a difference in Li content between these phases (cannot measure with EDS) causing a difference in contrast with SEM, (2) a solid-solution of sodalite with varying chemistries does not form in this system where phases with differing chemistries form and do not mix, or perhaps (3) an unknown heterogeneous interaction with the glass binder results in compositional variation in the sodalite.

For specimens with 10 mass\% SA800 fired at $650{ }^{\circ} \mathrm{C}$, the highest density was observed for SM2-AM$10 \%-650^{\circ} \mathrm{C}\left(84 \%\right.$ of $\left.\rho_{\mathrm{t}}\right)$ and this specimen had high sodalite at $97 \mathrm{mass} \%$. The highest densities for most were observed at the $750 \mathrm{C}$ firing temperature at $83-90 \%$ of $\rho_{\mathrm{t}}$ and the fractions of sodalite in these pellets were high (92-100 mass\%). Both the 850 and $950{ }^{\circ} \mathrm{C}$ firing temperatures worked equally well for sodalite production where all specimens had 100 mass $\%$ sodalite. However, it remains unclear why the densities of these pellets are all low $\left(68-84 \%\right.$ of $\left.\rho_{\mathrm{t}}\right)$. 


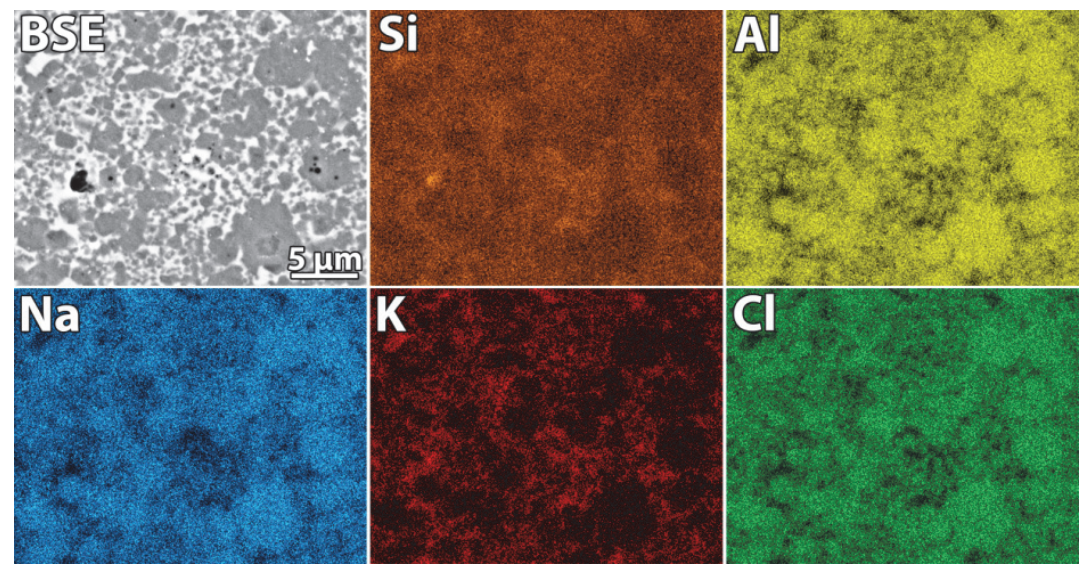

Figure 4.12. SEM-EDS dot map on SM2-5-C- $850^{\circ} \mathrm{C}$ showing compositional variations in the sodalite.
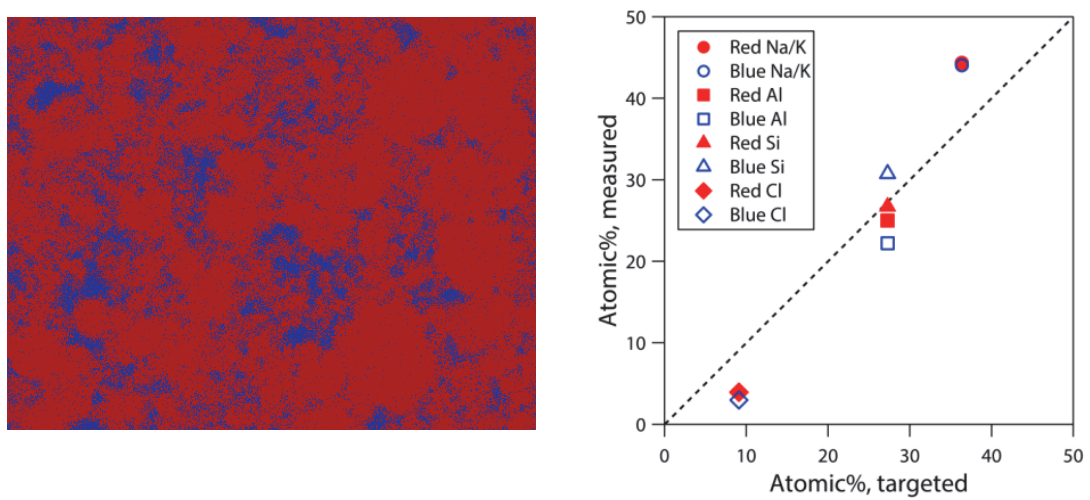

Figure 4.13. (left) Cluster phase analysis of the dot map seen in Figure 4.12 and (right) a comparison of the measured normalized compositions for the red and blue phase compared to the target values based on the target sodalite composition (oxygen is normalized out).

\subsubsection{SM-3 (Metakaolin) Overview}

The sodalite production in the SM3-AM and SM3-AM-C specimens was very low (3-59\%). However, even with only 5 mass $\%$ SA 800 , the sodalite production was increased by up to $72 \%$ in comparable formulations, e.g., SM3-5\%-650 ${ }^{\circ} \mathrm{C}(88 \%)$ vs. SM3-AM- $650^{\circ} \mathrm{C}(16 \%)$. All SM-3 formulations with the sintering aid showed unexpected highs for sodalite at the 650 and $750{ }^{\circ} \mathrm{C}$ firing temperatures, with most maxima at $750{ }^{\circ} \mathrm{C}$. One of the more anomalous data points was SM3-AM-5\% with 96 mass\% sodalite at $650^{\circ} \mathrm{C}$.

All specimens with 5 mass\% SA800 showed the same trend where the sodalite fraction decreased consistently between the $750-950{ }^{\circ} \mathrm{C}$ firing temperatures with very low sodalite fractions at the $950{ }^{\circ} \mathrm{C}$ firing temperatures (15-35\%); this was attributed to chloride volatility during firing. All of the sodalite fractions in the specimens with 10 mass\% SA800 were moderate to high (73-96\%). Even small quantities of sintering aid dramatically improved the sodalite production for SM-3 formulations.

The densities of specimens without sintering aids (SM3-AM and SM3-AM-C) were 52-69\% of $\rho_{\mathrm{t}}$ where the calcined specimens had a universally higher density than uncalcined specimens (10-22\%). On average, specimens with 5 mass $\%$ SA800 showed a noticeable improvement in density from those without the sintering aid (10\%) and the specimens with 10 mass\% SA800 showed a significant increase in density $(23 \%)$.

For specimens with 5 mass $\%$ SA 800 , those fired at 850 and $950{ }^{\circ} \mathrm{C}$ generally had the highest densities and for specimens with 10 mass\% SA800, the highest densities were achieved at the $950{ }^{\circ} \mathrm{C}$ firing 
temperature in all cases. For SM-3 specimens, an inverse relationship was observed between sodalite production and density at increased firing temperatures where low firing temperatures $\left(650-750{ }^{\circ} \mathrm{C}\right)$ produced pellets with high sodalite and high open porosity whereas high firing temperatures (850$950^{\circ} \mathrm{C}$ ) produced pellets with low sodalite and low open porosity.

\subsubsection{Comparisons}

The as-made specimens looked very similar with SEM (Figure 4.14). The particle sizes appeared to be somewhat consistent between samples with a mixture of both smaller and larger grains.

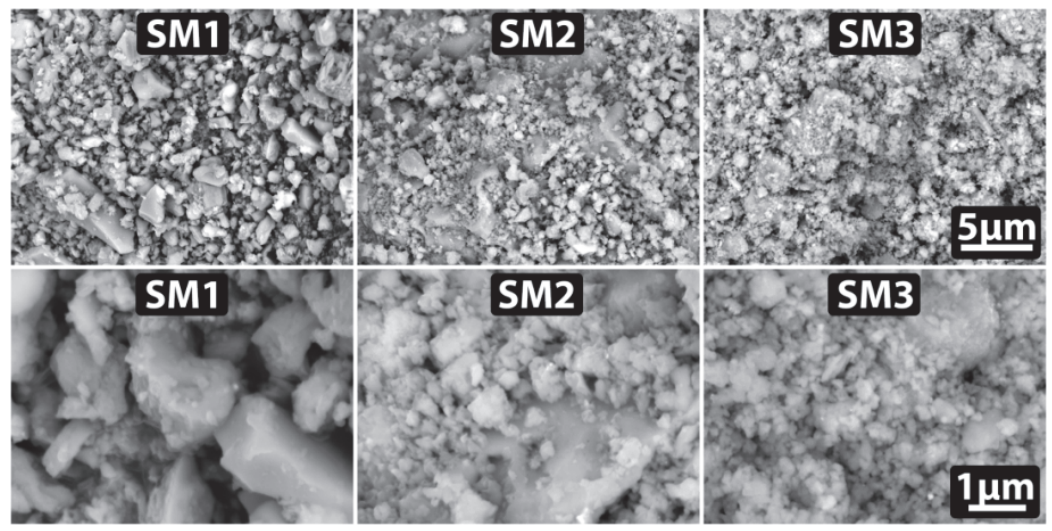

Figure 4.14. SEM micrographs showing a particle size comparison between the as-made powders for SM-1, SM-2, and SM-3 formulations.

Due to the large number of specimens generated during this study, the results were divided and compared in Figure 4.15-Figure 4.19. Figure 4.15 shows the differences in sodalite and density by both method and firing temperature and provides an overview of the variability seen between the three synthesis methods. For the SM1 and SM3 specimens, the highest densities were achieved at the 10 mass\% loading of the sintering aid, while the sintering aid additions proved less important for maximizing the density of SM2 specimens.
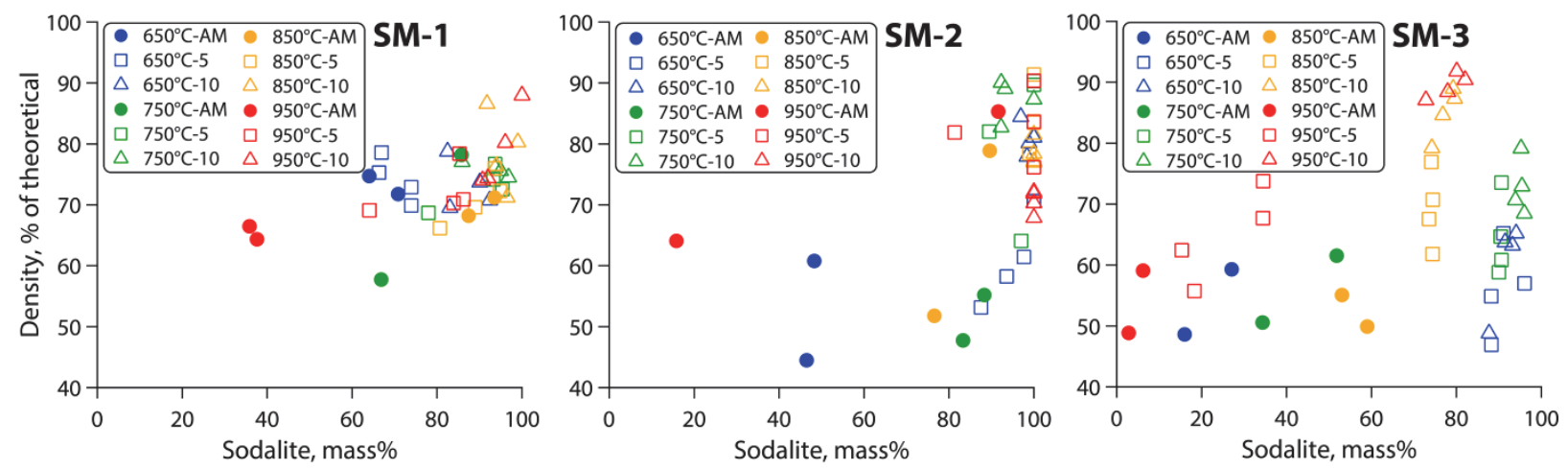

Figure 4.15. Comparison plots for density ( $\%$ of theoretical density) and the mass $\%$ sodalite of specimens sorted by method and heat-treatment temperature.

Figure 4.16 provides several comparisons between specimens of similar, but slightly different, preparations. For example, when considering the SM\#-5 and SM\#-AM-5 specimens (Comp. 1), where the only distinction between the two data sets is the time in the procedure at which the sintering aid is added (during the process or after drying, respectively), the sodalite fraction slightly improved when the sintering aid was added after drying. However, an overwhelming improvement to the density was observed when the sintering aid was added after drying. Comp. 2 provides a comparison for specimens 
where the timing of the calcination step varied and specimens were either calcined before (SM\#-C-5) or after (SM\#-5-C) the addition of the sintering aid. Comp. 2 shows that the sodalite fraction does not change much between these two different preparations but that the density varies widely. Comp. 3 and Comp. 4 are the same comparisons as Comp. 1 and Comp. 2 but with 10\% sintering aid instead of 5\% (by mass). Comp. 3 shows slightly the opposite trend as Comp. 1 where the sodalite fraction was higher in the SM\#-10 specimens versus the SM\#-AM-10 specimens. A similar trend was observed in Comp. 3 for the density. Comp. 4 shows that the sodalite fractions and densities are slightly higher for the SM\#-10-C specimens over the SM\#-C-10 specimens.
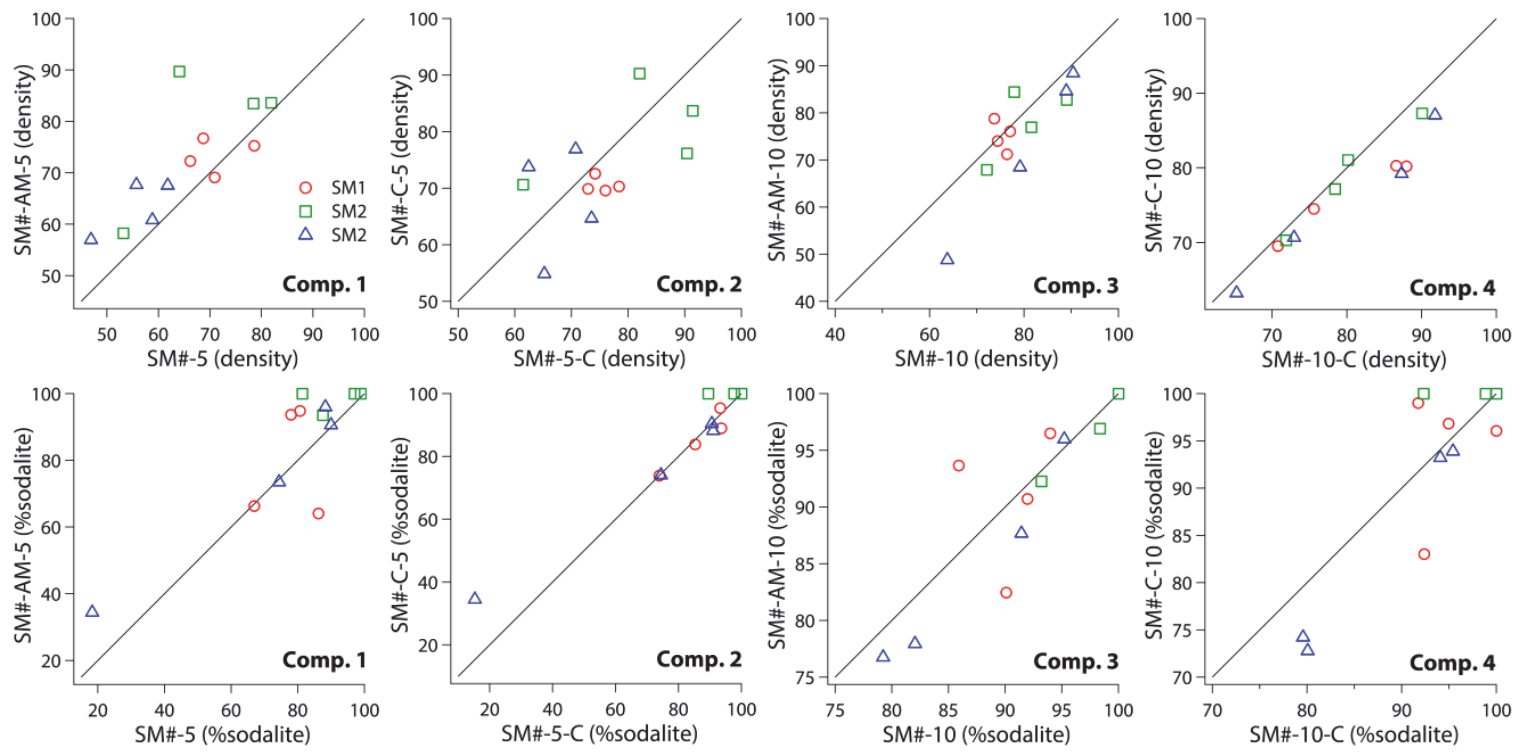

Figure 4.16. Comparison plots between like procedures for the density ( $\%$ of theoretical maximum for sodalite) and the mass $\%$ sodalite of pellets fired at all temperatures for specimens with 5 and 10 mass $\%$ additions of sintering aid (SA800).

Figure 4.17-Figure 4.19 provide comparisons between the calcined and uncalcined specimens. Figure 4.17 shows that all calcined specimens exhibited a significantly lower mass loss during firing than uncalcined specimens (up to $46 \times$ for SM1-C $-950^{\circ} \mathrm{C}$ vs. SM1 $-950^{\circ} \mathrm{C}$ ). In some cases, more mass loss was observed than would be expected, especially with most of the data points for the SM3 specimens where up to $17.2 \%$ mass loss was observed in calcined pellets (SM3-C-950 ${ }^{\circ} \mathrm{C}$ ).

Figure 4.18 shows the difference in density and sodalite between the as-made (SM\#-AM) and as-made and calcined (SM\#-AM-C) specimens. From this comparison it is easy to see that calcination improved both the density and sodalite fractions with minor exceptions. Figure 4.19 shows that calcination provides a marked increase in both sodalite and density, with a few exceptions. These results show the importance in calcination and are attributed to the release of bound hydrates from the sodalite structure during the calcination process. By allowing these hydrates to evolve from the sodalite prior to firing the pellets in pressed form, this should result in fewer voids than if the hydrates were allowed to evolve from pressed pellets. 

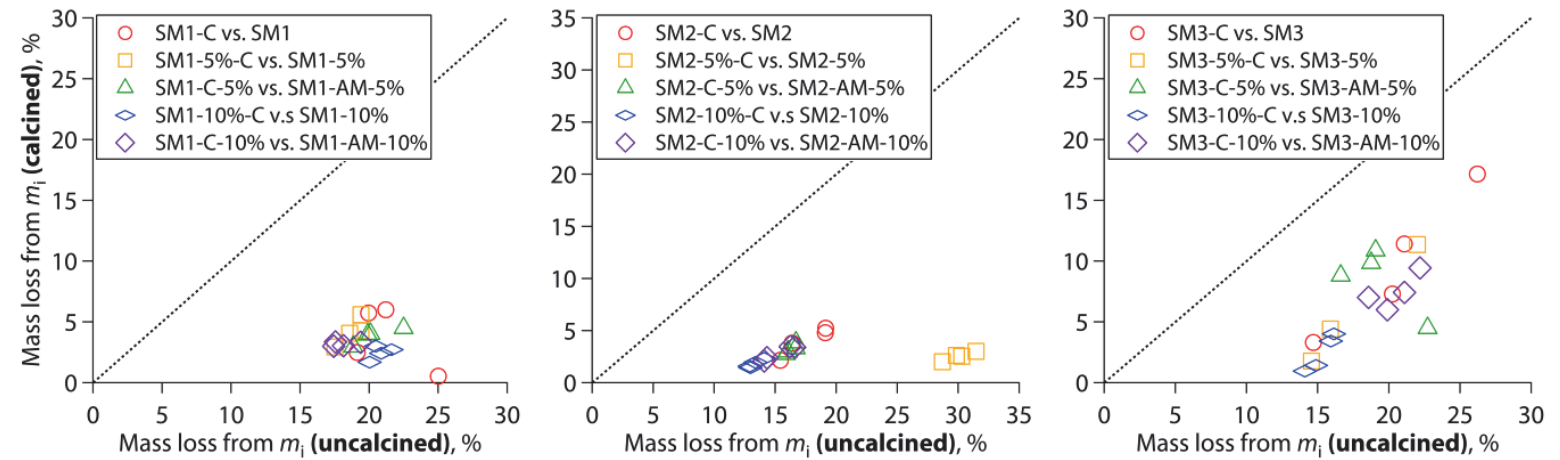

Figure 4.17. Comparison of mass loss during firing for calcined versus uncalcined specimens. Here, mass loss is determined by $100 \cdot\left(m_{\mathrm{i}}-m_{\mathrm{f}}\right) / m_{\mathrm{i}}$ (to get \%) where $m_{\mathrm{i}}$ is the initial mass and $m_{\mathrm{f}}$ is the final mass as measured on an analytical balance. Dotted lines are a guide for unity.
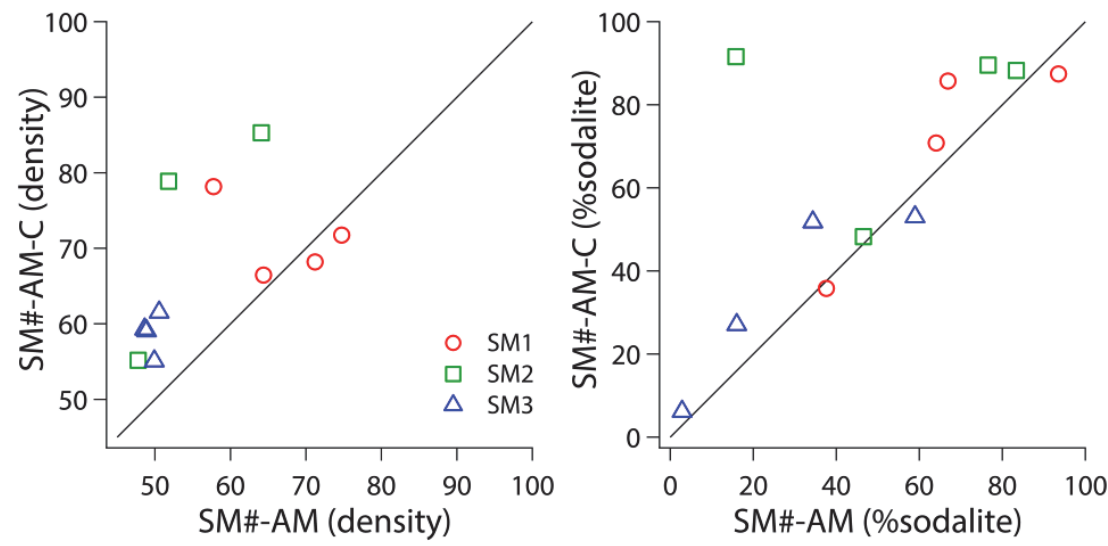

Figure 4.18. Comparison plots between like procedures for the density ( $\%$ of theoretical maximum for sodalite) and the mass $\%$ sodalite of as-made pellets without sintering aids fired at all temperatures (i.e., SM\#-AM and SM\#-AM-C). The $45^{\circ}$ lines are a guide for unity. 

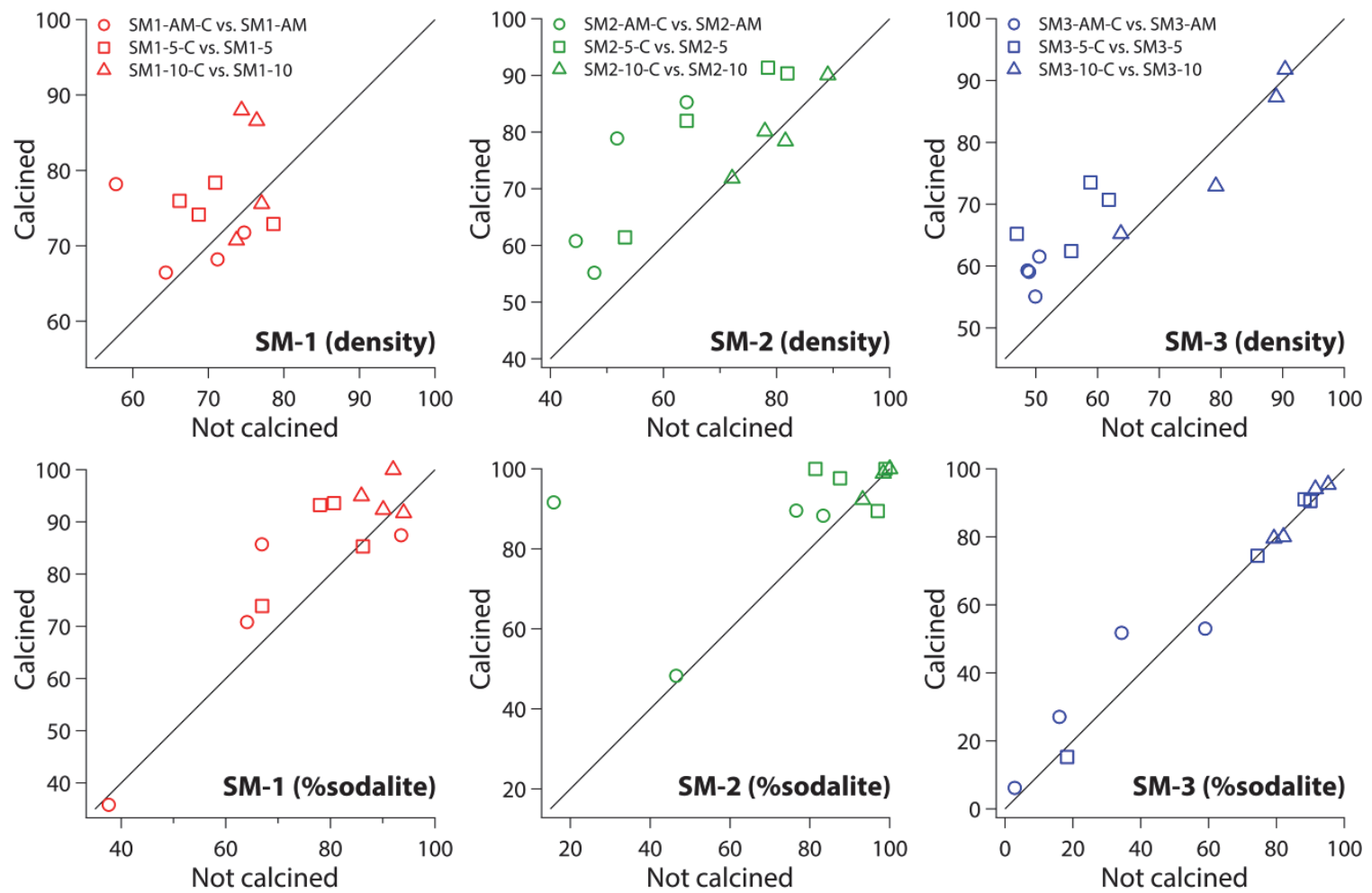

Figure 4.19. Comparison plots for the density ( $\%$ of theoretical density) and the mass $\%$ sodalite of specimens that were and were not calcined prior to being pressed into pellets (for all temperatures). Legends in the top row of figures are the same for the bottom row.

The results from the pressure variation study show that the pressure at which pellets were pressed had a slight effect on the SM-1 and SM-2 pellets but proved to be important for SM-3 pellets as seen in Figure 4.20 and Figure 4.21. For SM-2 and SM-3 pellets, the pellet density increased with pressure but with the SM-1 pellets, the trend was nearly decreasing. The sodalite fractions in all SM-1 and SM-2 pellets were $100 \%$ whereas the trend showed an increase for SM-3 pellets. These data show that high density and high sodalite pellets can be produced with the SM-2 method by simply pressing the SM2-5\%-C pellets uniaxially at moderate pressures. This result was not expected. Also, the data spread for all densities is low as with the sodalite fraction for SM-1 and SM-2 pellets, but not for sodalite for SM-3 pellets. Since these pellets are all pressed from the same batch that was homogenized prior to pressing pellets, this suggests heterogeneities in the SM-3 pellets. 

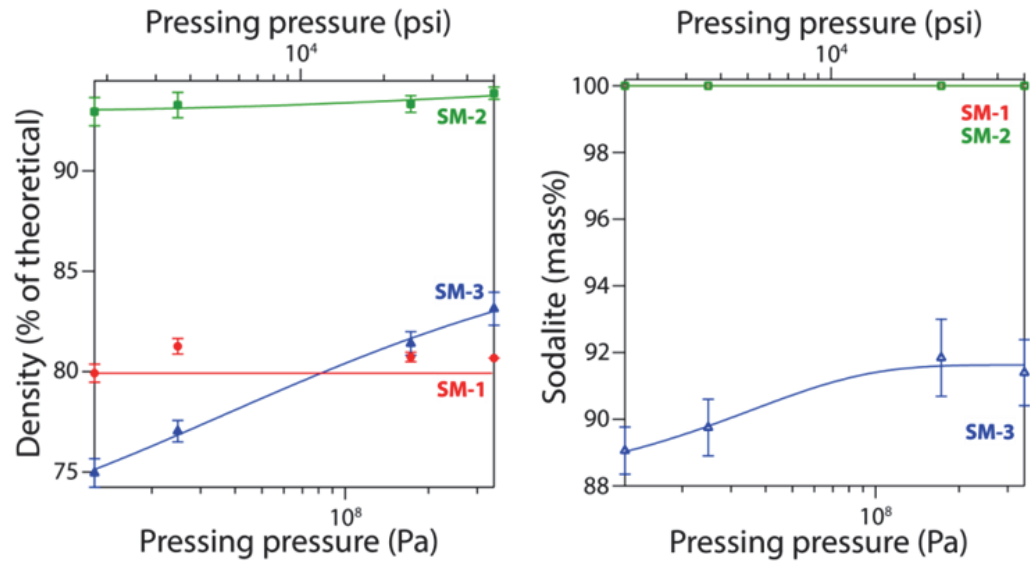

Figure 4.20. Comparisons from the pressure variation study showing the density and sodalite as a function of pressing pressure.

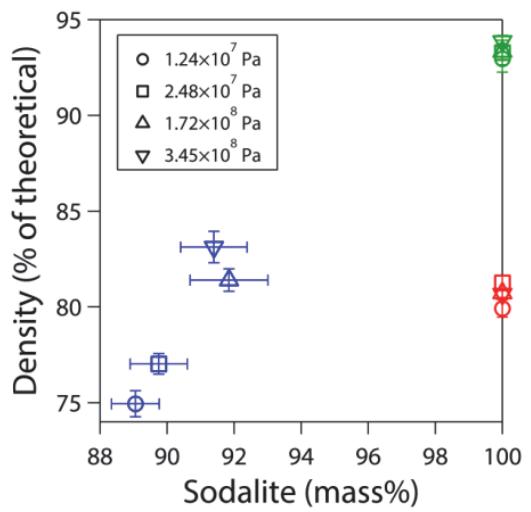

Figure 4.21. Density versus sodalite for the PVS samples. SM-1, SM-2, and SM-3 specimens are in red, green, and blue, respectively.

\subsubsection{Spark Plasma Sintering Results}

It is likely that hot isostatic pressing would further increase pellet densities from those seen in the pellet tests discussed above but that was not evaluated in the current work. Spark plasma sintering was evaluated but proved to be unsuccessful because the temperatures of the experiments $\left(526-554{ }^{\circ} \mathrm{C}\right)$ were not sufficient to convert the calcined product into pellets with high sodalite fractions. In the regularly fired specimens, the maximum sodalite fractions were observed in specimens fired at or above $750{ }^{\circ} \mathrm{C}$. The main limitation with this technique is that a dynamic force is applied during heating and if the heattreatment temperature is taken too high, the specimen could melt and be extruded from the die. We plan to further evaluate this technique with the TEOS-based sodalites that showed high sodalite fractions in the as-made, unfired products.

Figure 4.22 shows the temperature and die displacement as a function of time for each specimen. The values of displacement vary widely between specimens where the smallest displacement observed was in the SM-2 specimen which supports the data in Table 4.4 where this specimen showed the lowest increase in density between the fired and unfired measurements. The density of the fired SM-1 pellet was slightly higher than the calculated $\rho_{\mathrm{t}}$, suggesting no open porosity (Table 4.4), which is attributed to the large fraction of nepheline that has a higher density than sodalite at $2.5445 \times 10^{3} \mathrm{~kg} / \mathrm{m}^{3}$ (Table 4.5) (Dollase and Thomas, 1978a). This large fraction of nepheline is likely due to the loss of chloride during heating. 
It is possible that all of these have a fraction of unreacted, amorphous content as well since they were not doped with a standard prior to XRD. This is evidenced by the large fraction of unreacted salts in the SM1 and SM-2 pellets. Surprisingly, the phase distributions in the fired pellets varied widely with the SM-3 pellet showing the highest sodalite fraction (96\%) without any unreacted salts whereas the SM-1 pellet only had $28 \%$ sodalite; SM-2 was in between with $79 \%$ sodalite. It should be noted that the $\mathrm{NaCl}$ fractions reported for SM-1 and SM-2 are much higher than the amount of $\mathrm{NaCl}$ added to the batch and when considering that some sodalite formed in both cases, the true value of unreacted salt is actually much lower. The cause of this anomaly is currently not understood.
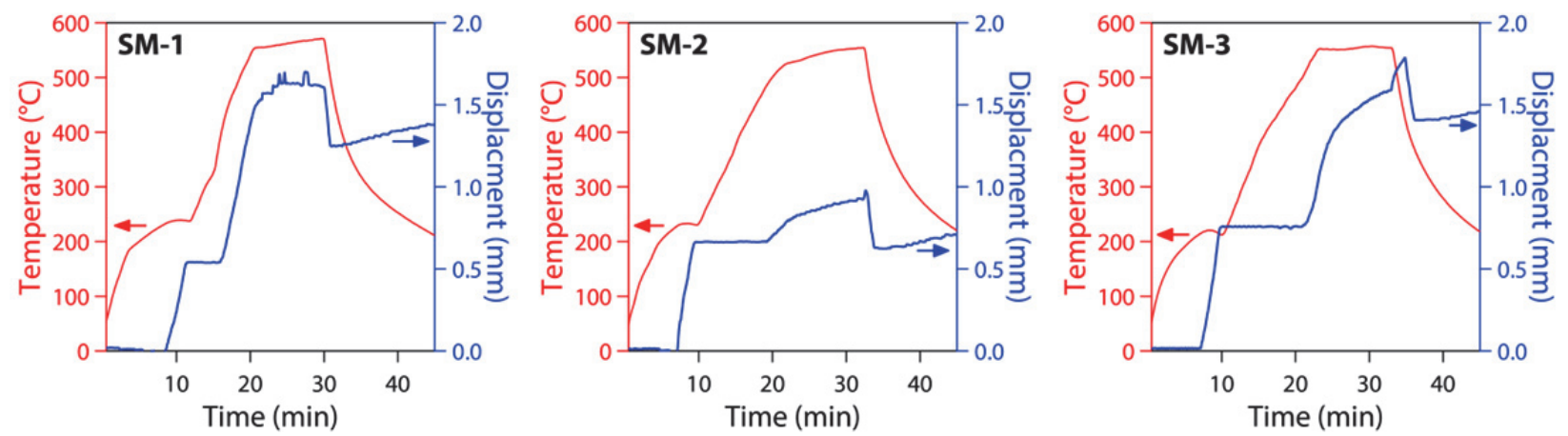

Figure 4.22. Spark plasma sintering results. These show the displacement and temperature as a function of time for each pellet.

Table 4.4. Density measurements for the specimens that were heated with spark plasma sintering. The data in parenthesis shows the percent of theoretical density for sodalite $\left(2.27 \times 10^{3} \mathrm{~kg} / \mathrm{m}^{3}\right)$.

\begin{tabular}{ccccc}
\hline Pellet & $\begin{array}{c}\text { Green Density, } \\
\times 10^{3} \mathrm{~kg} / \mathrm{m}^{3} \\
(\text { Bulk })\end{array}$ & $\begin{array}{c}\text { Fired Density, } \\
\times 10^{3} \mathrm{~kg} / \mathrm{m}^{3} \\
(\text { Archimedes })\end{array}$ & $\begin{array}{c}\text { Fired Density, } \\
\times 10^{3} \mathrm{~kg} / \mathrm{m}^{3} \\
(\text { Bulk })\end{array}$ & $\begin{array}{c}\text { Increase in Fired } \\
\text { Density over } \\
\text { Green Density }\end{array}$ \\
\hline SM-1 & $1.70(75 \%)$ & $2.49(102 \%)$ & $2.49(102 \%)$ & $46.5 \%$ \\
SM-2 & $1.49(66 \%)$ & $1.75(77 \%)$ & $1.73(76 \%)$ & $16.9 \%$ \\
SM-3 & $1.34(59 \%)$ & $1.82(79 \%)$ & $1.77(77 \%)$ & $34.0 \%$ \\
\hline
\end{tabular}

Table 4.5. Phase distribution from XRD measurements.

\begin{tabular}{ll}
\hline Pellet & Phase distribution (mass $\%$ ) \\
\hline SM-1 & $51.35 \%$ nepheline, $28.01 \%$ sodalite, $20.64 \% \mathrm{NaCl}$ \\
SM-2 & $78.78 \%$ sodalite, $15.70 \% \mathrm{NaCl}, 5.49 \%$ nepheline \\
SM-3 & $95.97 \%$ sodalite, $4.03 \% \mathrm{KAlO}_{2}$ \\
\hline
\end{tabular}

\subsubsection{Chemical Durability Tests}

The specimens chosen for the PCT were SM1-5\%-C- $850^{\circ} \mathrm{C}$, SM2-5\%-C-850 ${ }^{\circ} \mathrm{C}$, and SM3-5\%-C-750 ${ }^{\circ} \mathrm{C}$. The $\rho_{\mathrm{b}}, \%$ of $\rho_{\mathrm{t}}$, and phase distribution of these specimens are listed in Table 4.6. The normalized release rates $\left(N L_{\mathrm{i}} \mathrm{s}\right)$ for the PCT leachates are summarized in Table 4.7. The release for a given element was consistent in some cases ( $\mathrm{Al}, \mathrm{Na}, \mathrm{Si}$ ) but was inconsistent for others $(\mathrm{B}, \mathrm{Cl}, \mathrm{K}, \mathrm{Li}$, and lanthanides). Some of these discrepancies are due to the non-sodalite phases present in the as-fired specimens (see Table 4.6). For example, the Cl-release was highest for SM-1 but that specimen had some free $\mathrm{NaCl}$. 
Table 4.6. Summary of characterizations for PCT specimens prior to the PCT.

\begin{tabular}{l|c|c|c|c|c|c}
\hline & & & \multicolumn{4}{|c}{ Phase distribution (mass\%) } \\
\cline { 5 - 7 } Specimen & $\begin{array}{c}\rho_{\mathrm{b}} \\
\left(\times 10^{3}\right. \\
\left.\mathrm{kg} / \mathrm{m}^{3}\right)\end{array}$ & $\begin{array}{c}\rho_{\mathrm{t}} \\
(\%)\end{array}$ & $\begin{array}{c}\text { Sodalite } \\
\text { (Tamazyan } \\
\text { et al., 1988) }\end{array}$ & $\begin{array}{c}\text { NaCl } \\
\text { (Srinivasa } \\
\text { and Sanyal, } \\
1990)\end{array}$ & $\begin{array}{c}\text { Neph.* } \\
\text { (Tait et al., } \\
2003 \mathrm{~b})\end{array}$ & $\begin{array}{c}\mathrm{KAlO}_{2} \\
\text { (Sokolowski } \\
\text { and Kotarba, } \\
1998)\end{array}$ \\
\hline $\mathrm{SM} 1-5 \%-\mathrm{C}-850^{\circ} \mathrm{C}$ & 1.83 & 75.98 & 93.60 & 6.40 & - & - \\
\hline $\mathrm{SM} 2-5 \%-\mathrm{C}-850^{\circ} \mathrm{C}$ & 2.06 & 91.39 & 100.00 & - & - & - \\
\hline $\mathrm{SM} 3-5 \%-\mathrm{C}-750^{\circ} \mathrm{C}$ & 1.86 & 73.54 & 90.58 & - & 5.05 & 4.37 \\
\hline
\end{tabular}

*"Neph." is off-stoichiometric nepheline with composition $\mathrm{K}_{1.6} \mathrm{Na}_{6} \mathrm{Al}_{7.49} \mathrm{Si}_{8.51} \mathrm{O}_{32}$

Table 4.7. Averaged $N L_{\mathrm{i}}\left(\mathrm{g} / \mathrm{m}^{2}\right)$ in leachates from PCT-B with standard deviations listed in parenthesis. *Ln denotes total lanthanides analyzed and includes $\mathrm{Ce}, \mathrm{La}, \mathrm{Nd}, \mathrm{Pr}$, and $\mathrm{Sm}$. Note that the release values for Ba were below detection limits for SM-1 and SM-2.

\begin{tabular}{l|c|c|c}
\hline Element & SM-1 & SM-2 & SM-3 \\
\hline \multirow{2}{*}{$\mathrm{Al}$} & 0.0906 & 0.0821 & 0.0918 \\
& $(0.0006)$ & $(0.0043)$ & $(0.0023)$ \\
\hline $\mathrm{B}$ & 0.248 & 0.345 & 7.47 \\
& $(0.004)$ & $(0.024)$ & $(0.49)$ \\
\hline \multirow{2}{*}{$\mathrm{a}$} & - & - & 0.050 \\
& - & - & $(0.003)$ \\
\hline \multirow{2}{*}{$\mathrm{Ll}$} & 0.158 & 0.0562 & 0.0681 \\
& $(0.002)$ & $(0.0024)$ & $(0.0033)$ \\
\hline \multirow{2}{*}{$\mathrm{Li}$} & 0.300 & 0.581 & 2.15 \\
& $(0.006)$ & $(0.027)$ & $(0.14)$ \\
\hline \multirow{2}{*}{$\mathrm{Ln} *$} & 0.583 & 1.53 & 8.26 \\
& $(0.007)$ & $(0.11)$ & $(0.62)$ \\
\hline \multirow{2}{*}{$\mathrm{Na}$} & 0.0244 & 0.355 & 0.0398 \\
& $(0.0016)$ & $(0.035)$ & $(0.0008)$ \\
\hline \multirow{2}{*}{$\mathrm{Si}$} & 0.182 & 0.201 & 0.162 \\
& $(0.002)$ & $(0.013)$ & $(0.010)$ \\
\hline
\end{tabular}

Each of these synthesis routes has advantages and disadvantages from a chemical durability standpoint. First, although SM2-5\%-C-850 ${ }^{\circ} \mathrm{C}$ showed high density and sodalite, some $N L_{\mathrm{i}} \mathrm{s}$ were higher for this specimen than for SM1-5\%-C-850 ${ }^{\circ} \mathrm{C}$ including: lanthanides $(14 \times), \mathrm{Li}(1.6 \times), \mathrm{Si}(0.99 \times), \mathrm{K}(0.93 \times), \mathrm{B}$ $(0.39 \times)$, and $\mathrm{Na}(0.10 \times)$. Some $N L_{\mathrm{i}} \mathrm{s}$ were noticeably higher for SM3-5\%-C-750 ${ }^{\circ} \mathrm{C}$ than $\mathrm{SM} 2-5 \%-\mathrm{C}-$ $850^{\circ} \mathrm{C}$ including $\mathrm{B}(22 \times)$, Li $(4.4 \times)$, and $\mathrm{K}(2.7 \times)$. This higher leaching with $\mathrm{SM} 3-5 \%-\mathrm{C}-750^{\circ} \mathrm{C}$ is thought to be due to ineffective mixing of the metakaolin during the synthesis process. Additionally, the incongruent release of the elements from the sintering aid component could be due to selective leaching of this phase around the sodalite grain boundaries. This selective leaching could likely be reduced by adding an equally low-viscosity sintering aid with higher chemical durability.

The summary $N L_{\mathrm{i}}$ values for the rinsates are presented in Table 4.8. These results show that some soluble species were removed during the rinsing step. The specific surface areas measured with BET for SM1$5 \%-\mathrm{C}-850^{\circ} \mathrm{C}, \mathrm{SM} 2-5 \%-\mathrm{C}-850^{\circ} \mathrm{C}$, and SM3-5\%-C-750 ${ }^{\circ} \mathrm{C}$ were 79,9 , and $104 \mathrm{~m}^{2} / \mathrm{g}$, which equates to $33.2 \times, 3.8 \times$, and $45.9 \times$ the assumed values based on the procedure (ASTM C 1285-08, 2008), respectively. The $N L_{\mathrm{i}} \mathrm{s}$ adjusted for these measured specific surface areas are presented in Table 4.9; due 
to the low porosity measured with BET for SM2-5\%-C- $850^{\circ} \mathrm{C}$, the adjusted $N L_{\mathrm{i}} \mathrm{s}$ are higher than for SM1$5 \%-\mathrm{C}-850^{\circ} \mathrm{C}$ and $\mathrm{SM} 3-5 \%-\mathrm{C}-750^{\circ} \mathrm{C}$.

Table 4.8. Summary $N L_{\mathrm{i}}\left(\mathrm{m}^{2} / \mathrm{g}\right)$ values of rinsate analysis.

\begin{tabular}{l|l|l|l}
\hline Element & SM-1 & SM-2 & SM-3 \\
\hline $\mathrm{Al}$ & 1.02 & 0.856 & 2.77 \\
$\mathrm{~B}$ & 0.798 & 0.450 & 3.06 \\
$\mathrm{Cl}$ & 1.70 & 0.549 & 0.138 \\
$\mathrm{~K}$ & 1.38 & 0.984 & 3.20 \\
$\mathrm{Li}$ & 1.20 & 1.09 & 5.18 \\
$\mathrm{Ln}$ & 0.809 & 0.710 & 1.03 \\
$\mathrm{Na}$ & 1.53 & 1.19 & 2.71 \\
$\mathrm{Si}$ & 0.975 & 0.879 & 2.42 \\
\hline
\end{tabular}

Table 4.9. $N L_{\mathrm{i}}$ values $\left(\times 10^{-3} \mathrm{~g} / \mathrm{m}^{2}\right)$ adjusted for measured specific surface areas (standard deviations are below the average values).

\begin{tabular}{l|c|c|c}
\hline Element & SM1 & SM2 & SM3 \\
\hline \multirow{2}{*}{$\mathrm{Al}$} & 2.733 & 21.77 & 1.998 \\
& $(0.019)$ & $(1.15)$ & $(0.049)$ \\
$\mathrm{B}$ & 7.490 & 91.43 & 162.5 \\
& $(0.120)$ & $(6.37)$ & $(10.8)$ \\
$\mathrm{Cl}$ & 4.776 & 14.92 & 1.481 \\
& $(0.056)$ & $(0.65)$ & $(0.071)$ \\
$\mathrm{K}$ & 9.061 & 154.0 & 46.81 \\
& $(0.192)$ & $(7.1)$ & $(2.95)$ \\
$\mathrm{Li}$ & 17.60 & 406.8 & 179.8 \\
& $(0.21)$ & $(27.9)$ & $(13.5)$ \\
$\mathrm{Ln}$ & 17.60 & 406.8 & 179.8 \\
& $(0.21)$ & $(27.9)$ & $(13.5)$ \\
$\mathrm{Na}$ & 5.489 & 53.19 & 3.523 \\
& $(0.061)$ & $(3.30)$ & $(0.207)$ \\
$\mathrm{Si}$ & 2.036 & 35.45 & 1.833 \\
& $(0.025)$ & $(0.63)$ & $(0.039)$ \\
\hline
\end{tabular}

\subsection{Tellurite Glasses}

\subsubsection{Tellurite Glasses: Compositional Variation Study}

\subsubsection{X-Ray Diffraction}

To reiterate from the FY2010 report, before the PCT, all ten "P-series" tellurite glasses were determined by XRD to be bulk amorphous; in other words, if crystals were present in some of the glasses it was 
below the detection limit of the instrument (Riley et al., 2010). Figure 4.23 provides fitted backgrounds for the amorphous pre-PCT XRD spectra, which help reveal the subtle differences in peak location and intensity. The $\mathrm{PbO}$ glasses had the tallest and narrowest amorphous humps, suggesting that they were the most "structured" of this glass set.

Simulated amorphous diffraction patterns were generated for the different $\alpha-\mathrm{TeO}_{2}$ and $\beta-\mathrm{TeO}_{2}$ PDF patterns in the ICSD that matched diffraction peaks for the various glasses with Jade 6 software with a set crystallite size of $10 \mathrm{~nm}$ and $0 \%$ strain (see Table 4.10). The three simulated patterns differed in amorphous hump location and intensity as seen in the bottom portion of Figure 4.23. When observing Figure 4.23 (primarily the low-2 $\theta$ hump) with the vertical guides as a reference, it is apparent that some of the glasses seem to fit the simulated orthorhombic $\beta-\mathrm{TeO}_{2}$ phase (PDF\# 74-1131, Table 4.10), such as the $\mathrm{Al}_{2} \mathrm{O}_{3} / \mathrm{B}_{2} \mathrm{O}_{3}$-containing glasses $(\mathrm{P}-7$ " $\mathrm{Al} / \mathrm{B}$ " and $\mathrm{P}-2$ " $\mathrm{Al} / \mathrm{B}+\mathrm{Cl}$ "), while the other glasses seemed to fit with the tetragonal $\alpha-\mathrm{TeO}_{2}$ phases (PDF\#s 42-1365 and 78-1713, Table 4.10).

A summary of the XRD results for the ten glasses after the PCT is shown in Figure 4.23b with the best-fit structures presented in Table 4.10. Diffraction peaks matching $\mathrm{Pb}_{2} \mathrm{Te}_{3} \mathrm{O}_{7}$ crystals were observed in P-8 "Pb" and $\mathrm{P}-11$ " $\mathrm{Pb}+\mathrm{Cl}$ " glasses after the $\mathrm{PCT}$, although quantities were close to the detection limit for $\mathrm{P}-$ 11. For the tungstate glasses, only $\mathrm{P}-12$ "W+Cl" crystallized (to $\mathrm{Ba}_{3} \mathrm{WO}_{6}$ ) after the PCT. The other three families, the $\mathrm{Al}_{2} \mathrm{O}_{3} / \mathrm{B}_{2} \mathrm{O}_{3}$ (P-7 and P-2), $\mathrm{ZnO}$ (P-4 and P-13)-, and $\mathrm{P}_{2} \mathrm{O}_{5}$ (P-14 and P-5)-containing glasses all had characteristically different behavior with and without added $X \mathrm{Cl}_{20}$. Without $X \mathrm{Cl}_{20}$, all formed some $\alpha-\mathrm{TeO}_{2}$ (tetragonal paratellurite), while the $\mathrm{Zn}$ glass (P-4) also formed some $\mathrm{Zn}_{2} \mathrm{Te}_{3} \mathrm{O}_{8}$. After adding $X \mathrm{Cl}_{20}$, all formed some $\beta-\mathrm{TeO}_{2}$ (orthorhombic tellurite), while $\mathrm{Al} / \mathrm{B}+\mathrm{Cl}(\mathrm{P}-2)$ also formed $\mathrm{Na}_{2} \mathrm{Te}_{2} \mathrm{O}_{7}$, and $\mathrm{Zn}+\mathrm{Cl}(\mathrm{P}-13)$ formed $\mathrm{Zn}_{2} \mathrm{Te}_{3} \mathrm{O}_{8}$.

It should be noted that of the glasses that showed crystalline $\mathrm{TeO}_{2}$ phases after the PCT (i.e., P-7 "Al/B," $\mathrm{P}-2$ "Al/B+Cl", P-5 "P”, P-14 "P+Cl", P-4 "Zn", and P-13 "Zn+Cl"), those containing $X \mathrm{Cl}_{20}$ always formed $\beta-\mathrm{TeO}_{2}$, whereas those without $X \mathrm{Cl}_{20}$ were shown to form $\alpha-\mathrm{TeO}_{2}$. This demonstrates that mixedchloride additions to the tellurite glass network have a consistent effect on the network structure. In these systems, it appears that $X \mathrm{Cl}_{20}$ additions, or perhaps the alkali and lanthanide elements in the presence of chlorine, favor the formation of $\beta-\mathrm{TeO}_{2}$ structures. 

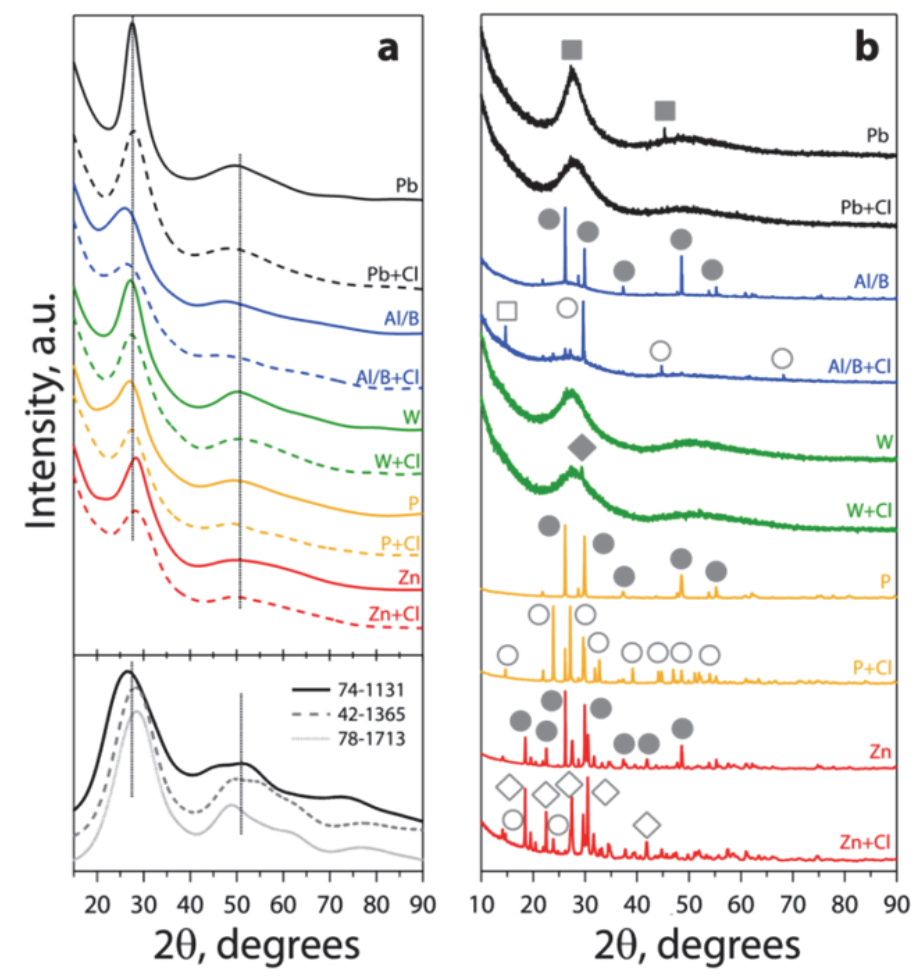

Figure 4.23. (a) XRD summary plot showing the amorphous background fits for all glasses before PCT for comparison with three amorphous simulated patterns for $\mathrm{TeO}_{2} \mathrm{PDF}$ structures (74-1131, 42-1365, and 78-1713; see Table 4.10). Vertical lines are added as a visual guide for the location of the primary amorphous humps. The spectra are vertically offset (intensities retained) to accentuate the differences so the vertical scale is listed in arbitrary units (a.u.). (b) XRD spectra for all 10 glasses after PCT. Best-fit diffraction peaks were identified and are denoted by symbols near the most intense of the diffraction peaks for each phase observed for each glass. Here, - denotes tetragonal $\alpha-\mathrm{TeO}_{2}, \mathrm{O}$ denotes orthorhombic $\beta-\mathrm{TeO}_{2}$, denotes $\mathrm{Pb}_{2} \mathrm{Te}_{3} \mathrm{O}_{7}, \square$ denotes $\mathrm{Na}_{2} \mathrm{Te}_{2} \mathrm{O}_{7}, \diamond$ denotes $\mathrm{Ba}_{3} \mathrm{WO}_{6}$, and $\diamond$ denotes $\mathrm{Zn}_{2} \mathrm{Te}_{3} \mathrm{O}_{8}$. This figure was recreated from our previous work (Riley et al., 2010). 
Table 4.10. Summary of phases identified in the tellurite glasses for the composition-variation study.

\begin{tabular}{|c|c|c|c|c|c|}
\hline Phase ID & PDF\# & ICSD\# & SG (SG\#) & Reference & Glasses \\
\hline$\alpha-\mathrm{TeO}_{2}$ & $78-1713$ & 62897 & $P 43212(96)$ & (Kondratyuk et al., 1987) & P-4 “Zn”, P-7 “Al/B” \\
\hline$\alpha-\mathrm{TeO}_{2}$ & $42-1365$ & 27515 & P41212 (92) & & P-5 “P (phosphorus)” \\
\hline$\beta-\mathrm{TeO}_{2}$ & $74-1131$ & 26844 & Pbca (61) & (Beyer, 1967) & $\begin{array}{l}\mathrm{P}-2 \text { " } \mathrm{Al} / \mathrm{B}+\mathrm{Cl} ", \mathrm{P}-14 \\
\text { "P+Cl", } \mathrm{P}-13 \text { " } \mathrm{Zn}+\mathrm{Cl} \text { " }\end{array}$ \\
\hline $\mathrm{Pb}_{2} \mathrm{Te}_{3} \mathrm{O}_{7}$ & 43-0549 & N/A & $F m-3 m(225)$ & (Kulcu, 1987) & $\begin{array}{l}\mathrm{P}-8 \text { "Pb", } \mathrm{P}-11 \\
\text { "Pb+Cl”" }\end{array}$ \\
\hline $\mathrm{Na}_{2} \mathrm{Te}_{2} \mathrm{O}_{7}$ & $35-1263$ & N/A & $\operatorname{Imm} 2(44)$ & (Knop and Demazeau, 1981) & $\mathrm{P}-2$ “Al/B+Cl” \\
\hline $\mathrm{Ba}_{3} \mathrm{WO}_{6}$ & $33-0182$ & $\mathrm{~N} / \mathrm{A}$ & $F m-3 m(225)$ & & $\mathrm{W}-12$ “W+Cl” \\
\hline $\mathrm{Zn}_{2} \mathrm{Te}_{3} \mathrm{O}_{8}$ & $89-4454$ & 50705 & $C 2 / c(15)$ & (Feger et al., 1999) & $\mathrm{P}-13$ “ $\mathrm{Zn}+\mathrm{Cl} "$ \\
\hline
\end{tabular}

(a) Blanchard, F., Department of Geology, University of Florida, Gainesville (1990).

(b) National Bureau of Standards (U.S.) Monogr. 25, 19, p 21 (1982).

N/A: not applicable

\subsubsection{Raman Spectroscopy}

Raman spectroscopy was performed to approach the tellurite glass structure from a different angle than other possible techniques such as XRD. Raman addresses the length scale that is below XRD and will ID short range symmetrical structures at the nearest and next nearest neighbor level.

Figure 4.24 shows example results for the Raman curve fitting for the lead-tellurite "P-series" glasses. Assignments for tellurite bands A through $\mathbf{E}$ have been made according to Table 3.13 with structural representations of these units derived from the literature and presented in Figure A.1 (Appendix A). Raman spectra for the specimens with and without $X \mathrm{Cl}_{20}$ are shown in Figure 4.25. Peak wavenumbers $(\tilde{v})$ and areal intensity determined from the fitting are shown in Table 4.11. In all cases except the PbOcontaining glasses $\left(\mathrm{P}-8\right.$ " $\mathrm{Pb}$ " and $\mathrm{P}-11$ " $\mathrm{Pb}+\mathrm{Cl}$ "), $\mathbf{A}$ and $\mathbf{B}$ bands assigned to $\mathrm{TeO}_{3}$ and $\mathrm{TeO}_{3+1}$ tp groups could not be distinguished, so they were assigned to $\mathbf{A}$, the higher frequency band. In only two cases, $\mathrm{P}_{2} \mathrm{O}_{5}$-containing and $\mathrm{PbO}$-containing glasses, could a $\mathbf{D}$ band, assigned to Te-O asymmetric stretch in $\mathrm{TeO}_{2}$, be distinguished. A summary of peak fitting for the different glass systems is presented below.

- PbO-glasses ( $\mathrm{P}-8$ " $\mathrm{Pb}$ " and $\mathrm{P}-11$ "Pb+Cl"). Fitting all of the tellurite bands $\mathbf{A}(t p), \mathbf{B}(t p), \mathbf{C}(t b p)$, $\mathbf{D}(t b p)$, and $\mathbf{E}(\mathrm{Te}-\mathrm{O}-\mathrm{Te})$ was required.

- $\quad \mathbf{A l}_{2} \mathbf{O}_{3} / \mathbf{B}_{2} \mathbf{O}_{3}$-glasses (P-7 “Al/B" and P-2 “Al/B+Cl”). Spectra were fitted with $\mathbf{A}(t p), \mathbf{C}(t b p)$, and $\mathbf{E}$ (Te-O-Te) tellurite bands. Additional bands were observed at $\sim 840 \mathrm{~cm}^{-1}$ and, in the case without chlorides, at $\sim 1300 \mathrm{~cm}^{-1}$. Both of the aforementioned bands were assigned to B-O-B(III) in pyroborate structures, though the latter band has been also applied to $\mathrm{Al}(\mathrm{IV})$ substituting for $\mathrm{B}(\mathrm{IV})$ in the borate network (Brow et al., 1997; Li et al., 2003).

- $\quad \mathbf{W O}_{3}$-glasses (P-9 "W" and P-12 "W+Cl"). The fit of current data incorporated tellurite bands $\mathbf{A}(t p)$, $\mathbf{C}(t b p)$, and $\mathbf{E}$ (Te-O-Te). In addition, another band was observed in the $\mathbf{E}$ envelope (408-412 $\mathrm{cm}^{-1}$ ) distinct from the $\sim 360 \mathrm{~cm}^{-1}$ band, which is also visible in the spectra (Figure $4.25 \mathrm{c}$ ) and previously assigned to deformation vibrations of $\mathrm{WO}_{6}$ (Chowdari and Kumari, 1999; Sekiya et al., 1994a). In the fitting procedure, the three apparent bands in the 300-600 $\mathrm{cm}^{-1}$ region were fit with only two bands as shown in Table 4.11. Bands $\sim 860$ and $\sim 930 \mathrm{~cm}^{-1}$ were assigned to W-O stretches internal and external to the clusters, respectively (see Table 3.13).

- $\quad \mathbf{P}_{2} \mathbf{O}_{5}$-glasses (P-5 "P" and P-14 "P+Cl"). Glasses in the current study were fit with six Raman bands, including four of the tellurite bands: $\mathbf{A}(t p), \mathbf{C}(t b p), \mathbf{D}(t b p)$, and $\mathbf{E}$. Raman fitting required two peaks in the envelope of the $\mathbf{E}$ peak assigned to the Te-O-Te bending vibrations (see Table 3.13). Normally the P-O-P bending vibrations are at lower wavenumbers, i.e., $\tilde{v}<300 \mathrm{~cm}^{-1}$, but it is possible 
that some band mixing occurred to cause the asymmetry in this first peak. One or two weak peaks around $1000 \mathrm{~cm}^{-1}$ can be ascribed to $\mathrm{PO}_{4}$ groups with one or two non-bridging oxygens.

- ZnO-glasses (P-4 "Zn" and P-13 "Zn+Cl”). Spectra in the current study were adequately fit with only three tellurite bands: A ( $t p), \mathbf{C}(t b p)$, and $\mathbf{E}$ (Te-O-Te bending).
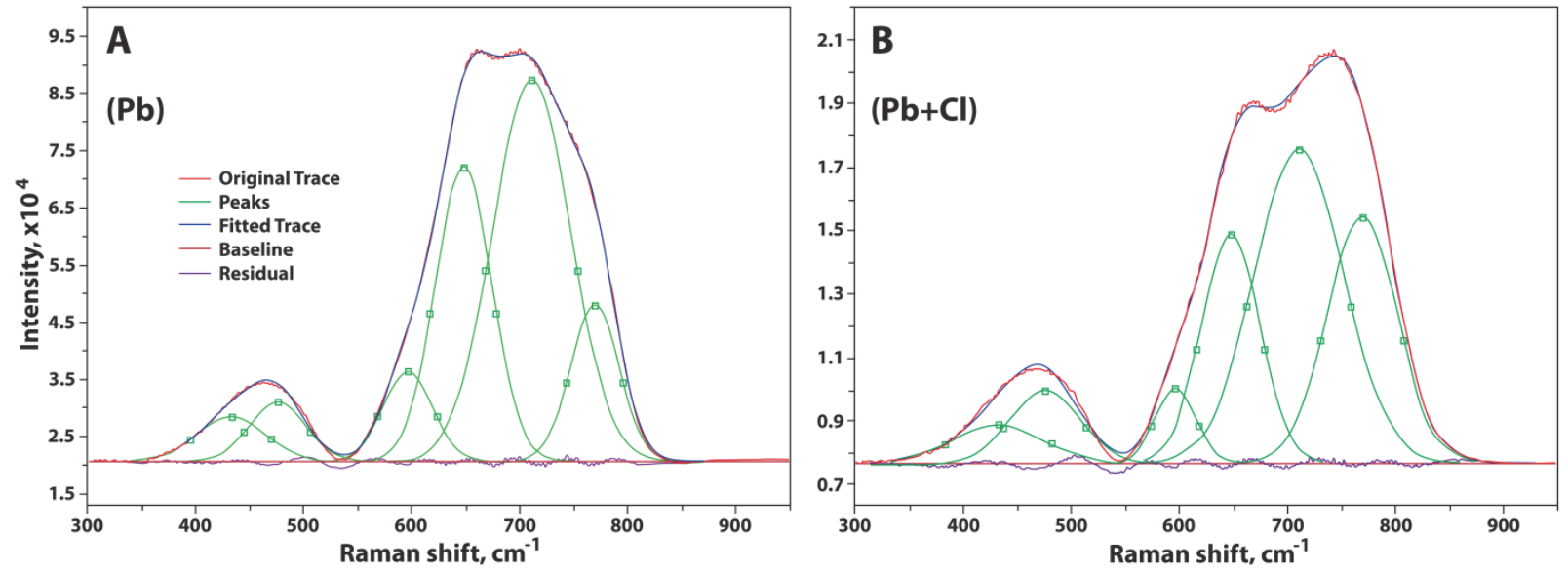

Figure 4.24. Examples of curve fitting for $(\mathrm{A}) \mathrm{Pb}(\mathrm{P}-8)$ and $(\mathrm{B}) \mathrm{Pb}+\mathrm{Cl}(\mathrm{P}-11)$ glasses.
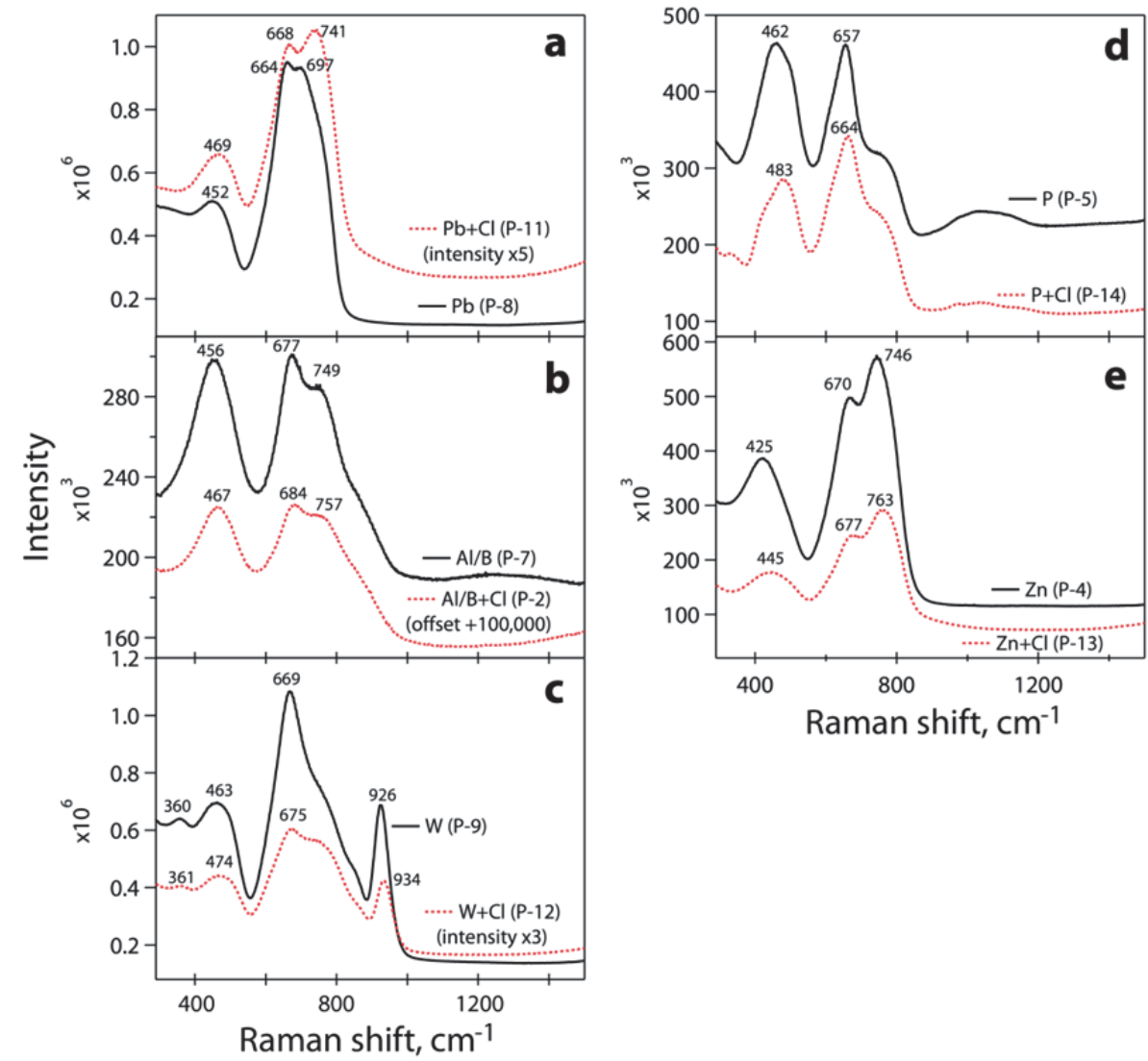

Figure 4.25. Raw Raman spectra without backgrounds removed for glasses with and without $X \mathrm{Cl}_{20}$ (listed as "Cl"). Spectra are not offset but are shown as collected unless otherwise noted. (a) PbO-glasses P-8 (bottom, black) and P-11 (top, red, intensity 5× from measured); (b) $\mathrm{Al}_{2} \mathrm{O}_{3} / \mathrm{B}_{2} \mathrm{O}_{3}$-glasses P-7 (top, black) and P-2 (bottom, red, offset $+100,000$ counts); (c) $\mathrm{WO}_{3}$-glasses P-9 (top, black) and P-12 (bottom, red, intensity $3 \times$ from measured); (d) $\mathrm{P}_{2} \mathrm{O}_{5}$-glasses P-5 (top, black) and P-14 (bottom, red); and (e) $\mathrm{ZnO}$ glasses P-4 (top, black) and P-13 (bottom, red). 


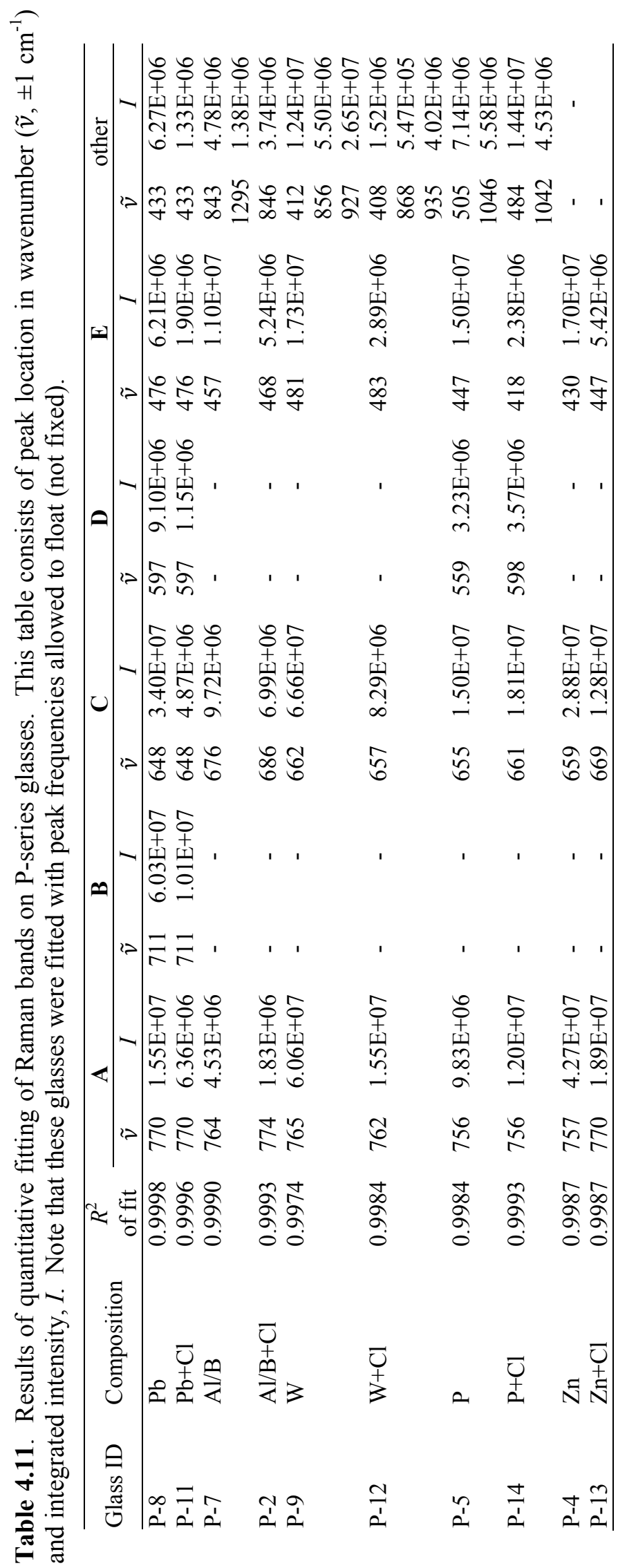


As a rough estimate of the fraction of $t p$ units as compared to $t b p$ units $\left(\mathrm{TeO}_{3} / \mathrm{TeO}_{4}\right)$ in the glass, areal intensity ratios were taken from the fitted spectra of the intensity of the $\mathbf{A}$ band to that of the $\mathbf{C}$ band, i.e., $I(\mathbf{A}$ band $) / I(\mathbf{C}$ band) (Chowdari and Kumari, 1999; Himei et al., 1994). These corresponding ratios are shown in Table 4.12. It should be noted that these ratios seem to imply similar Raman cross-sections between the various bands, which might not necessarily be the case. Because it was not always clear whether the bands in the region (i.e., $720-780 \mathrm{~cm}^{-1}$ ) should be assigned to $\mathbf{A}$ or $\mathbf{B}$, the intensity ratio of $\mathbf{A}+\mathbf{B}$ to the $\mathbf{C}$ band, i.e., $I(\mathbf{A}+\mathbf{B}$ bands $) / I(\mathbf{C}$ band $)$, was also analyzed. Only the data for the PbO-containing glasses $\mathrm{P}-8(\mathrm{~Pb})$ and $\mathrm{P}-11(\mathrm{~Pb}+\mathrm{Cl})$ were influenced by this approach.

Table 4.12. Ratios of key tellurite peaks from the Raman data. Since "B" was only observed in the $\mathrm{Pb}$-containing glasses, the ratio $I(\mathbf{A}+\mathbf{B}) / I(\mathbf{C})$ is only different from $I(\mathbf{A}) / I(\mathbf{C})$ for these glasses.

\begin{tabular}{llcccl}
\hline Glass ID & Composition & $I(\mathbf{A}) / I(\mathbf{C})$ & $I(\mathbf{A}+\mathbf{B}) / I(\mathbf{C})$ & $\begin{array}{c}\text { Mole\% oxide } \\
\text { additive }\end{array}$ & $\begin{array}{c}\text { Crystalline phases } \\
\text { after PCT }\end{array}$ \\
\hline $\mathrm{P}-8$ & $\mathrm{~Pb}$ & 0.46 & 2.23 & 16.8 & $\mathrm{PbTe}_{3} \mathrm{O}_{7}$ \\
$\mathrm{P}-11$ & $\mathrm{~Pb}+\mathrm{Cl}$ & 1.31 & 3.38 & 13.1 & $\mathrm{PbTe}_{3} \mathrm{O}_{7}$ \\
$\mathrm{P}-7$ & $\mathrm{~A} 1 / \mathrm{B}$ & 0.47 & 0.47 & 29.5 & $\alpha-\mathrm{TeO}_{2}$ \\
$\mathrm{P}-2$ & $\mathrm{Al} / \mathrm{B}+\mathrm{Cl}$ & 0.26 & 0.26 & 24.9 & $\beta-\mathrm{TeO}_{2}, \mathrm{Na}_{2} \mathrm{Te}_{2} \mathrm{O}_{7}$ \\
$\mathrm{P}-9$ & $\mathrm{~W}$ & 0.91 & 0.91 & 18.7 & $\mathrm{None}$ \\
$\mathrm{P}-12$ & $\mathrm{~W}+\mathrm{Cl}$ & 1.87 & 1.87 & 14.5 & $\mathrm{Ba}_{3} \mathrm{WO}_{6}$ \\
$\mathrm{P}-5$ & $\mathrm{P}$ & 0.66 & 0.66 & 10.0 & $\alpha-\mathrm{TeO}_{2}$ \\
$\mathrm{P}-14$ & $\mathrm{P}+\mathrm{Cl}$ & 0.66 & 0.66 & 7.9 & $\beta-\mathrm{TeO}_{2}$ \\
$\mathrm{P}-4$ & $\mathrm{Zn}$ & 1.48 & 1.48 & 30.1 & $\alpha-\mathrm{TeO}_{2}, \mathrm{Zn}_{2} \mathrm{Te}_{3} \mathrm{O}_{8}$ \\
$\mathrm{P}-13$ & $\mathrm{Zn}+\mathrm{Cl}$ & 1.48 & 1.48 & 24.5 & $\beta-\mathrm{TeO}_{2}, \mathrm{Zn}_{2} \mathrm{Te}_{3} \mathrm{O}_{8}$ \\
\hline
\end{tabular}

As stated by Himei et al., all of these band ratios should be properly corrected by a $y$-intercept when against $\mathrm{O} / \mathrm{Te}$ on a molar basis (Himei et al., 1994). However, we elected not to follow this approach because we were more interested in comparing the influence of primary dopants and the presence of mixed chlorides on the structural variation(s) between glasses in each binary/ternary system. Glasses with $I(\mathbf{A})<I(\mathbf{C})$ should have more $\mathrm{TeO}_{4}$ units, and those with ratios $>1$ should have more $\mathrm{TeO}_{3}$ units. Ratios that decrease in the $\mathrm{XCl}_{20}$-containing glass should indicate presence of more $\mathrm{TeO}_{4}$ than glasses without $X \mathrm{Cl}_{20}$, while ratios that increase should indicate more $\mathrm{TeO}_{3}$. Glasses with ratios $<1$ include almost all of the glasses: P-8 (Pb), P-11 ( Pb+Cl), P-7(Al/B), P-2 (Al/B+Cl), P-9 (W), P-5 (P), and P-14 $(\mathrm{P}+\mathrm{Cl})$. Glasses predicted to have primarily $\mathrm{TeO}_{3}$ units include $\mathrm{P}-12(\mathrm{~W}+\mathrm{Cl}), \mathrm{P}-4(\mathrm{Zn})$, and $\mathrm{P}-13$ $(\mathrm{Zn}+\mathrm{Cl})$. It is likely that results for P-9 $(\mathrm{W})$ and $\mathrm{P}-12(\mathrm{~W}+\mathrm{Cl})$ are influenced by peaks from the $\mathrm{W}$ network because, according to Sekiya et al., bands A, B, and $\mathbf{C}$ all have contributions from the Wnetwork modes while $\mathbf{E}$ contains only contributions from the Te network (Sekiya et al., 1994a). Therefore, it is difficult to comment on the P-9 $(\mathrm{W})$ and P-12 $(\mathrm{W}+\mathrm{Cl})$ with only the Raman spectra.

It was instructive to compare the ratios for the $\mathrm{Zn}$ glasses with those of the $\mathrm{Al} / \mathrm{B}$ glasses. These four glasses had nearly identical molar additions of oxides to the $\mathrm{TeO}_{2}$. However, the $\mathrm{Zn}$ glasses $(\mathrm{P}-4$ and $\mathrm{P}-$ 13) were highly depolymerized (i.e., the $\mathrm{TeO}_{4} t b p$ units have been changed to mostly $\mathrm{TeO}_{3} t p$ units), as observed in other studies. This is because the $\mathrm{ZnO}$ breaks the Te-O chains and only secondarily acts as a glass former. In Al/B glasses (P-7 and P-2), however, only $\mathrm{TeO}_{4}$ units are expected from $\mathrm{TeO}_{2}-\mathrm{B}_{2} \mathrm{O}_{3}$ systems (Rong et al., 1992). This leaves $8-9$ mole $\% \mathrm{Al}_{2} \mathrm{O}_{3}$, which creates $\sim 15 \%$ tp groups based on NMR of $\mathrm{TeO}_{2}-\mathrm{Al}_{2} \mathrm{O}_{3}$ (Sakida et al., 2001). Ignoring the role of $\mathrm{B}_{2} \mathrm{O}_{3}$, for $8-9$ mole $\% \mathrm{Al}_{2} \mathrm{O}_{3}$, one would estimate $\sim 45 \%$ Al in $\mathrm{AlO}_{6}, \sim 20 \%$ in $\mathrm{AlO}_{5}$, and $\sim 34 \%$ in $\mathrm{AlO}_{4}$, though it is unknown how the simultaneous presence of $\mathrm{B}_{2} \mathrm{O}_{3}$ would affect the $\mathrm{Al}$ speciation (Sakida et al., 2001). When adding $X \mathrm{Cl}_{20}$ to these glasses, the spectra for the P-13 $(\mathrm{Zn}+\mathrm{Cl})$ glass did not noticeably change while the ratios decreased for P-2 $(\mathrm{Al} / \mathrm{B}+\mathrm{Cl})$, indicating that even more $\mathrm{TeO}_{4}$ formed. These differences are important when considering the radically different behavior of the Zn glasses (P-5 and P-14) and Al/B glasses (P-7 
and $\mathrm{P}-2)$ in chemical durability tests. As shown Table 4.13, in P-4 ( $\mathrm{Zn}) \mathrm{P}-13(\mathrm{Zn}+\mathrm{Cl})$ glasses were very soluble, whereas the P-7 (Al/B) and P-2 $(\mathrm{Al} / \mathrm{B}+\mathrm{Cl})$ glasses were only slightly soluble, i.e., $\mathrm{P}-13(\mathrm{Zn}+\mathrm{Cl})$ showed very high $N L_{\mathrm{Na}}$ at $88.9 \mathrm{~g} / \mathrm{m}^{2}$ and $\mathrm{P}-2(\mathrm{Al} / \mathrm{B}+\mathrm{Cl})$ showed low $N L_{\mathrm{Na}}$ at $6.71 \mathrm{~g} / \mathrm{m}^{2}$.

For the $\mathrm{Pb}$ glasses, $\mathrm{P}-8$ and $\mathrm{P}-11$, the estimated fraction of $t p$ from NMR data in the literature is $\sim 33 \%$ for the $\sim 17 \mathrm{~mole} \% \mathrm{PbO}^{-\mathrm{TeO}_{2}}$ glass, which is similar to our glass without chlorides (Sakida et al., 2001). This suggests that the ratio $I(\mathbf{A}) / I(\mathbf{C})$ is more reliable than NMR. The value of this ratio is $<1(t b p>t p)$, while $I(\mathbf{A}+\mathbf{B}) / I(\mathbf{C})$ is $>1(t b p<t p)$. The fraction of $t p$ for P-8 $(\mathrm{Pb})$ glass estimated from NMR $(\sim 33 \%)$ is significantly greater than that estimated for the P-7 (A1/B) glass $(\sim 15 \% t p)$ but the amount of $t p$ clearly decreased in $\mathrm{P}-11(\mathrm{~Pb}+\mathrm{Cl})$ glass when chlorides were added.

Though P-5 $(\mathrm{P})$ and $\mathrm{P}-14(\mathrm{P}+\mathrm{Cl})$ glasses showed predominantly tbp structures, their chemical durability was very poor. The $\mathrm{P}+\mathrm{Cl}$ glass was phase-separated, the leachate from this glass was highly enriched in $\mathrm{P}$, and the residual solids were composed primarily of crystalline $\beta-\mathrm{TeO}_{2}$ (Riley et al., 2010). Kozhukharov et al. (1978) investigated the $\mathrm{TeO}_{2}-\mathrm{P}_{2} \mathrm{O}_{5}$ binary system for regions of miscibility/immiscibility and found that uniform glass was formed at 3-15 mole $\% \mathrm{P}_{2} \mathrm{O}_{5}$. However, many complex immiscible phases also occurred at various temperatures within this region. These results could explain the incongruent leaching observed during the PCT as well as the phase separation.

\subsubsection{Chemical Durability Analysis}

The chemical durability of the glasses studied here did not appear to fit an obvious trend and varied widely from $N L_{\mathrm{Na}}=0.478$ to $88.9 \mathrm{~g} / \mathrm{m}^{2}$ release over the course of the seven-day PCT (see Table 4.13). The variation was attributed to phase separation in some of the glasses and the ratio of $t p: t b p$ structural units. The $\mathrm{Pb}+\mathrm{Cl}(\mathrm{P}-11)$ glass showed the highest chemical durability, $\mathrm{W}+\mathrm{Cl}(\mathrm{P}-12)$ and $\mathrm{Al} / \mathrm{B}+\mathrm{Cl}(\mathrm{P}-2)$ were only somewhat durable, and $\mathrm{P}+\mathrm{Cl}(\mathrm{P}-14)$ and $\mathrm{Zn}+\mathrm{Cl}(\mathrm{P}-13)$ showed poor chemical durability. The reduced chemical durability in the $\mathrm{Al} / \mathrm{B}+\mathrm{Cl}(\mathrm{P}-2)$ and $\mathrm{P}+\mathrm{Cl}(\mathrm{P}-14)$ glasses was likely due to phase separation, which was clearly visible in these glasses with the naked eye (Riley et al., 2010).

Table 4.13. Summary of normalized release results from seven-day PCT-B for P-series tellurite glasses with $\mathrm{XCl}_{20}$. More details are provided in our earlier work such as ranges/deviations (Riley et al., 2010).

\begin{tabular}{lccccc} 
Glass ID & Composition & $N L_{\mathrm{Li}}\left(\mathrm{g} / \mathrm{m}^{2}\right)$ & $N L_{\mathrm{Na}}\left(\mathrm{g} / \mathrm{m}^{2}\right)$ & $N L_{\mathrm{K}}\left(\mathrm{g} / \mathrm{m}^{2}\right)$ & $N L_{\mathrm{Cl}}\left(\mathrm{g} / \mathrm{m}^{2}\right)$ \\
\hline $\mathrm{P}-11$ & $\mathrm{~Pb}+\mathrm{Cl}$ & 1.00 & 0.478 & 0.298 & 0.528 \\
$\mathrm{P}-2$ & $\mathrm{Al} / \mathrm{B}+\mathrm{Cl}$ & 5.50 & 6.72 & 3.45 & 3.85 \\
$\mathrm{P}-12$ & $\mathrm{~W}+\mathrm{Cl}$ & 7.25 & 10.3 & 2.85 & 4.62 \\
$\mathrm{P}-14$ & $\mathrm{P}+\mathrm{Cl}$ & 82.7 & 86.0 & 88.4 & 30.3 \\
$\mathrm{P}-13$ & $\mathrm{Zn}+\mathrm{Cl}$ & 85.3 & 88.9 & 89.1 & 84.7 \\
\hline
\end{tabular}

Although the $\mathrm{Pb}+\mathrm{Cl}$ glass, $\mathrm{P}-11$, performed very well in the $\mathrm{PCT}$, it had a $I(\mathbf{A}) / I(\mathbf{C})$ ratio similar to that of the $\mathrm{Zn}+\mathrm{Cl}$ glass $(\mathrm{P}-13)$, which performed the worst of all the glasses studied $\left(N L_{\mathrm{Na}}=88.9 \mathrm{~g} / \mathrm{m}^{2}\right)$, implying similar tp:tbp fractions in these two glasses (see Table 4.12). However, since the Pb glasses, P-8 and P11 , also had a $\mathbf{B}$ band present adding to the $t p$ signal $(>60 \%)$, the fraction of $t p$ units in the $\mathrm{Pb}+\mathrm{Cl}$ glass was the highest of the glasses studied, i.e., $I(\mathbf{A}+\mathbf{B}) / I(\mathbf{C})$. This suggests that perhaps the $t p$ structure is necessary to increase chemical durability, although more work is needed to verify the difference between the $\mathbf{A}$ and $\mathbf{B}$ bands since the $\mathbf{B}$ band did not appear in any of the glasses other than the $\mathrm{Pb}$ glasses and seem to contribute significantly to the $t p$ unit signal.

The $\mathrm{Al} / \mathrm{B}+\mathrm{Cl}$ glass, $\mathrm{P}-2$, was the second-best-performing $X \mathrm{Cl}_{20}$-loaded glass, with $N L_{\mathrm{Na}}=6.72 \mathrm{~g} / \mathrm{m}^{2}$. However, the $\mathrm{Al} / \mathrm{B}+\mathrm{Cl}$ and $\mathrm{Pb}+\mathrm{Cl}(\mathrm{P}-11)$ glasses were an order of magnitude apart in $N L_{\mathrm{Na}}$ and the leaching appeared very different in each glass. The leaching in the Al/B-glasses, P-7 and P-2, appeared incongruent, likely due to the fact that the $\mathrm{Al} / \mathrm{B}+\mathrm{Cl}$ glass was visibly phase-separated before the PCT; also, the PCT leachate was enriched in $\mathrm{B}$ and $\mathrm{Cl}$. Interestingly, the total dissolved $\mathrm{Al}$ species measured in 
the PCT leachates from both the Al/B-glasses was very low (Riley et al., 2010). Also, the dissolved B species measured in the PCT leachates for the $\mathrm{Al} / \mathrm{B}$ glass was nearly double $(1.855 \times)$ that in the $\mathrm{Al} / \mathrm{B}+\mathrm{Cl}$ glass, whereas the amount of $\mathrm{B}_{2} \mathrm{O}_{3}$ present in the $\mathrm{Al} / \mathrm{B}+\mathrm{Cl}$ glass was not very different from that in the $\mathrm{Al} / \mathrm{B}$ glass (P-7, 8.1 versus 9.0 mass\%) (Riley et al., 2010).

\subsubsection{Thermal analysis}

The P-8 $(\mathrm{Pb})$ and $\mathrm{P}-11(\mathrm{~Pb}+\mathrm{Cl}) \mathrm{P}$-series lead-tellurite glasses were studied with the TA Instruments SDT Q600 DTA-TGA and the results are presented in Table 4.14 and Figure 4.26. The $T_{\mathrm{g}}$ of $\mathrm{P}-8\left(\sim 291^{\circ} \mathrm{C}\right)$ is comparable to a typical tellurite glass and when 10 mass $\%$ of $X \mathrm{Cl}_{20}$ was added to P-8 to produce the P-11 glass, the $T_{\mathrm{g}}$ was reduced to $\sim 254{ }^{\circ} \mathrm{C}$ for the $10{ }^{\circ} \mathrm{C} / \mathrm{min}$ ramp rate. The temperature stability of these glasses can be increased by incorporating additional components into the glass, such as $\mathrm{GeO}_{2}$ and $\mathrm{Al}_{2} \mathrm{O}_{3}$; these options are currently under investigation (Hocdé et al., 2004).

Table 4.14. Summary of DTA-TGA results for P-8 and P-11 run at both 10 and $15{ }^{\circ} \mathrm{C} / \mathrm{min}$ ramp heating rates.

\begin{tabular}{|c|c|c|c|c|c|c|c|}
\hline $\begin{array}{l}\text { Glass } \\
\text { ID }\end{array}$ & $\begin{array}{l}\text { Rate, } \\
{ }^{\circ} \mathrm{C} / \mathrm{min}\end{array}$ & $\begin{array}{c}\text { Mass, } \\
\text { mg }\end{array}$ & $\begin{array}{l}T_{\mathrm{g}}, \\
{ }^{\circ} \mathrm{C}\end{array}$ & Peak ID & $\begin{array}{c}T_{\mathrm{x}} \text { (onset), } \\
{ }^{\circ} \mathrm{C}\end{array}$ & $\begin{array}{c}T_{\mathrm{c}}(\max ), \\
{ }^{\circ} \mathrm{C}\end{array}$ & $\begin{array}{l}T_{\mathrm{M}}, \\
{ }^{\circ} \mathrm{C}\end{array}$ \\
\hline \multirow{4}{*}{ P-8 } & \multirow{2}{*}{10} & \multirow{2}{*}{75.549} & \multirow{2}{*}{290.53} & (1) & 321.37 & 334.42 & 524.22 \\
\hline & & & & (2) & 371.98 & 387.26 & 620.10 \\
\hline & \multirow{2}{*}{15} & \multirow{2}{*}{67.482} & \multirow{2}{*}{288.68} & (1) & 321.00 & 334.15 & 522.09 \\
\hline & & & & (2) & 374.79 & 390.59 & 619.90 \\
\hline \multirow{4}{*}{ P-11 } & \multirow{2}{*}{10} & \multirow{2}{*}{34.805} & \multirow{2}{*}{253.90} & (1) & 392.36 & 417.02 & 536.38 \\
\hline & & & & (2) & - & - & - \\
\hline & \multirow{2}{*}{15} & \multirow{2}{*}{33.378} & \multirow{2}{*}{264.00} & (1) & 326.79 & 340.06 & 542.38 \\
\hline & & & & (2) & 366.59 & 395.03 & \\
\hline
\end{tabular}




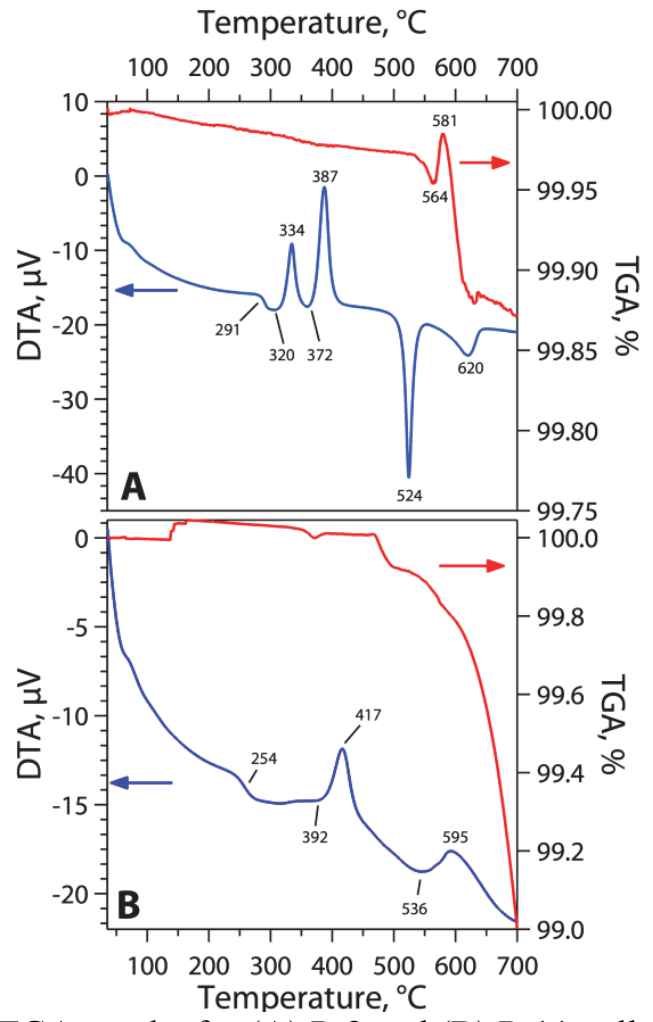

Figure 4.26. DTA/TGA results for (A) P-8 and (B) P-11 collected during heating.

In addition, the thermal diffusivity was measured for P-11 with a NETZSCH LFA-457. In order to perform these measurements, discs of the specimens were prepared from cylinders cast in fused quartz molds (see Figure 3.3). The thermal diffusivity for P-11 was measured at $0.23-0.28 \times 10^{-6} \mathrm{~m}^{2} / \mathrm{s}$ and these results are presented in Figure 4.27a. We did prepare a disc of P-8 for thermal diffusivity measurements, although the glass tends to crystallize when we try to pour and anneal cylinders that can be cut into discs (see Figure 4.27b). These crystals would make it difficult to accurately measure the thermal diffusivity for the glassy phase.
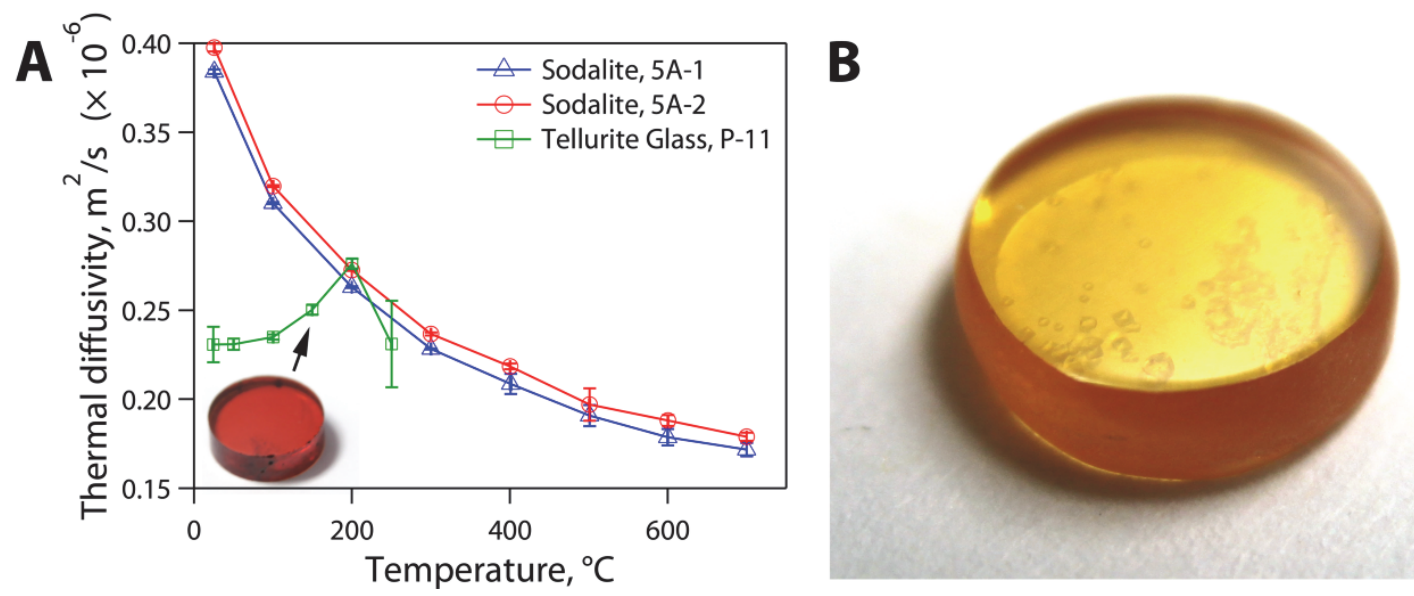

Figure 4.27. (A) Thermal diffusivity, $\alpha$, for tellurite glass, P-11, as well as PNNL Sodalite, 5A, as a function of temperature. (B) A P-8 specimen prepared for LFA measurements ( $\sim 1 \mathrm{~cm}$ in diameter); it is slightly crystallized (see small, clear $\mathrm{Pb}_{2} \mathrm{Te}_{3} \mathrm{O}_{7}$ crystals throughout the bulk). 


\subsubsection{Tellurite Glasses: Lead-Tellurite and Structural Variation Glasses Study}

\subsubsection{Visual Appearance}

The lead-tellurite glasses appeared very different from one another; $\mathrm{TPb}_{0}$ was a transparent yellow-orange glass; $\mathrm{TPb}_{5}, \mathrm{TPb}_{10}, \mathrm{TPb}_{12.5}$, and $\mathrm{TPb}_{14}$ were transparent red glasses; $\mathrm{TPb}_{15}$ was phase-separated; and $\mathrm{TPb}_{17.5}, \mathrm{TPb}_{22.5}$, and $\mathrm{TPb}_{30}$ were dark yellow-white and opaque ceramic-like in appearance (Figure 4.28). Phase separation was observed at $15 \% \mathrm{XCl}_{20}$-loading, although not at $14 \%$. Thus, the $\mathrm{XCl}_{20}$-loading limit was determined to be between $14 \%$ and $15 \%$ in this lead tellurite system $\left(\mathrm{TeO}_{2}: \mathrm{PbO}=3.545\right.$, by mass $)$.

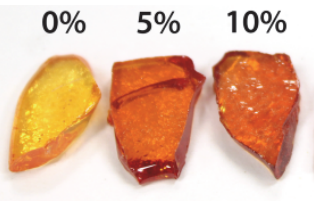

(a)
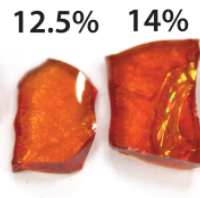

$15 \%$

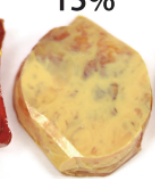

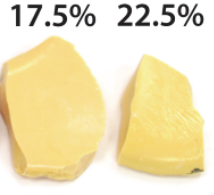

$1 \mathrm{~cm}$

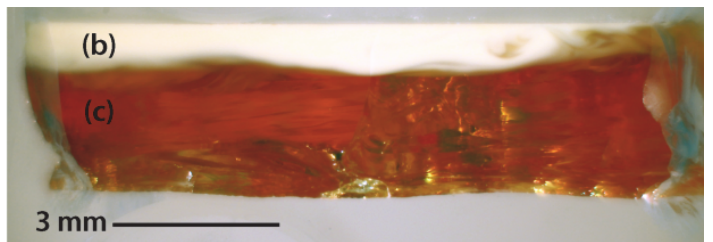

Figure 4.28. (a) Pictures of lead-tellurite glasses at different waste loadings. (b,c) A polished crosssection of the two phases present in the phase-separated T2G-1-PbO specimen (Riley et al., 2010). Note that $\mathrm{TPb}_{30}$ is not shown but looks identical to $\mathrm{TPb}_{22.5}$.

The structural variation set of glasses are presented in Figure 4.29. While these glasses were quite different in color, the crystalline/amorphous nature varied only slightly; some made very nice glasses (e.g., T2G-33 and T2G-34) while others were highly crystalline (e.g., T2G-21b-d). All of these glasses had very low viscosities during melting/pouring. For all of the lead-tellurite glasses, a temperature of $700{ }^{\circ} \mathrm{C}$ was sufficient for melting. For the ZnO-based glasses, T2G-29a and T2G-29b, a higher temperature of $730{ }^{\circ} \mathrm{C}$ was required for melting. Some volatilization was observed for T2G-29a, T2G-32, and T2G-33 whereas no volatilization was observed for T2G-27a, T2G-27b, or T2G-34.

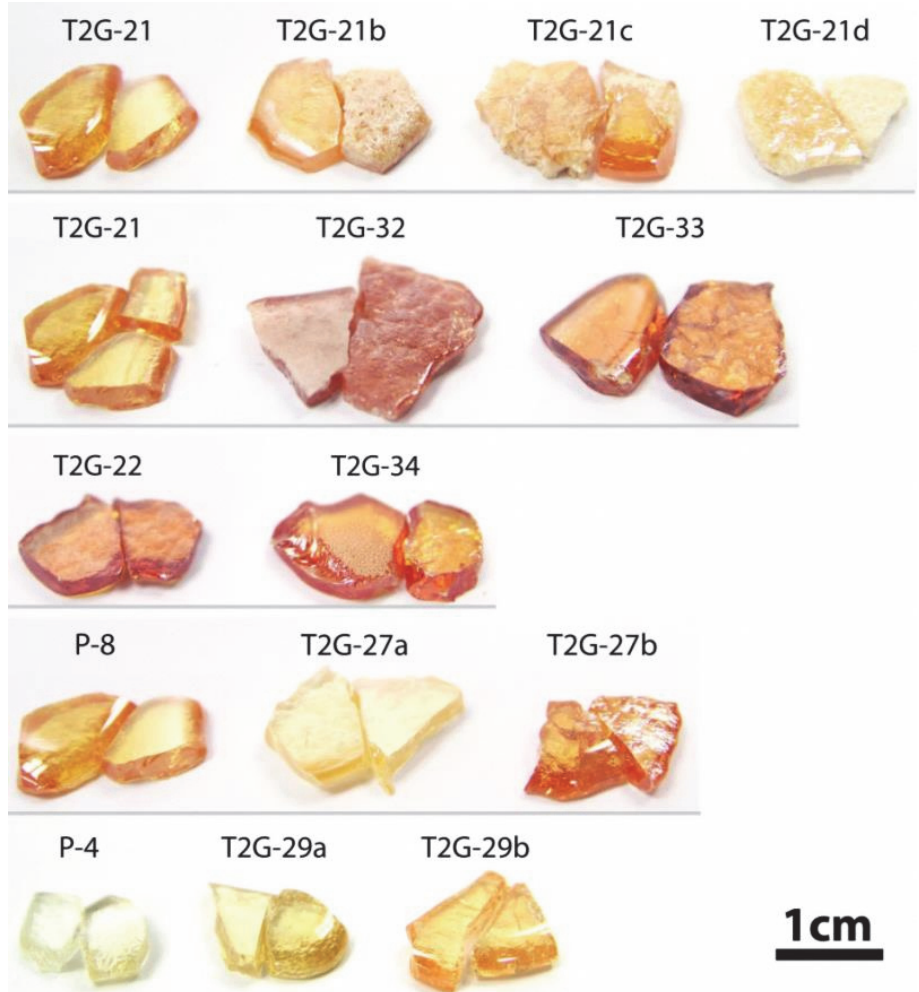

Figure 4.29. Summary figure providing visual comparisons between the structural variation glasses. Here, T2G-21 was used as a placeholder for P-8 since they have identical compositions and appearances. 


\subsubsection{Density Analysis}

Adding $\mathrm{XCl}_{20}$ to the baseline lead-tellurite glasses decreased the density, as one would expect considering that the density of the waste components, $\rho_{\text {salt }}$, is lower than that of the glass, $\rho_{\mathrm{b}}$, at $<3 \times 10^{3} \mathrm{~kg} / \mathrm{m}^{3} \mathrm{vs}$. $>6 \times 10^{3} \mathrm{~kg} / \mathrm{m}^{3}$, respectively (see Table 3.4 and Figure 4.30a). This does not account for a density difference between the individual phases present in the phase-separated glasses. Figure $4.30 \mathrm{~b}$ shows the storage densities $\left(\rho_{\mathrm{sd}}\right)$ of the different glasses as functions of waste loading. The concept of $\rho_{\text {sd }}$ is presented here to show the large difference between the volumetric waste loading of the tellurite glasses compared to that of the baseline glass-bonded sodalite $\left(163 \mathrm{~kg} / \mathrm{m}^{3}\right)$. A waste form with a high $\rho_{\text {sd }}$ is preferred because it will require a smaller volume to store an equivalent mass of waste. For comparison, the final glass-bonded sodalite baseline waste form for the Echem waste is $\sim 90 \%$ of its theoretical density with $\rho_{\mathrm{b}}=2170 \mathrm{~kg} / \mathrm{m}^{3}, \sim 7.5$ mass $\%$ waste loading, giving $\rho_{\mathrm{sd}}=163 \mathrm{~kg} / \mathrm{m}^{3}$.
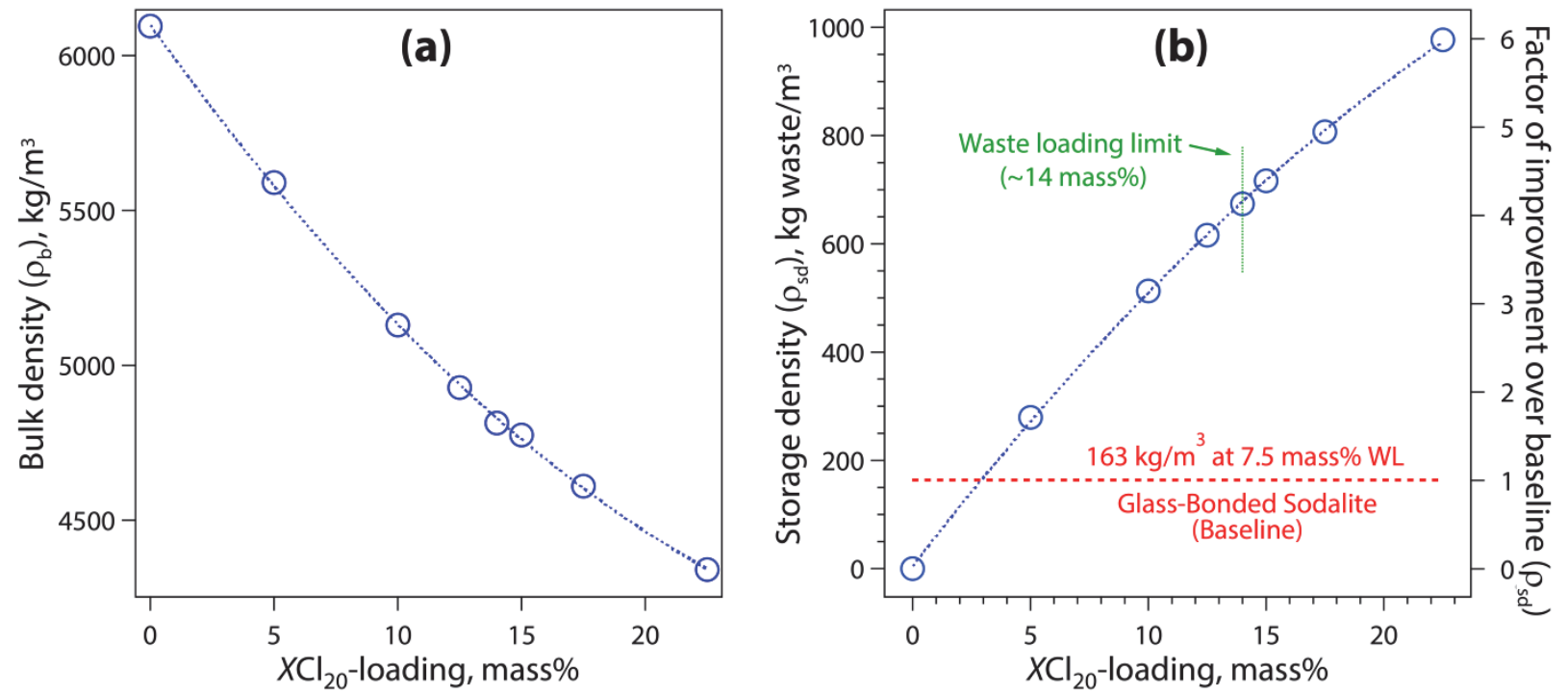

Figure 4.30. (a) Bulk density $\left(\rho_{\mathrm{b}}\right)$ and (b) storage density $\left(\rho_{\text {sd }}\right)$ of the lead-tellurite glasses as a function of $X \mathrm{Cl}_{20}$-loading. In (b) a comparison is given to the baseline glass-bonded sodalite.

\subsubsection{Raman Analysis}

Micro-Raman analysis of the two phases in T2G-1-PbO (Figure 4.28) revealed that, qualitatively, the B $(t p)$ band was substantially larger than the $\mathbf{C}(t b p)$ band in phase (a) when compared to phase (b), as seen in Figure 4.31-see Table 3.13 for band assignments. This implies that the $t b p$ units are more prevalent in phase (b), suggesting that alkali and/or chlorine enrichment in phase (a) leads to more depolymerization of the tellurite network (i.e., more $t p \mathrm{TeO}_{3}$ or $\mathrm{TeO}_{3+1}$ units). The strongest, cleanest micro-Raman signal of the white phase was analyzed quantitatively as well. Microscopy and XRD did not indicate that the white phase was crystalline, but it could have been phase-separated on a very small scale. 


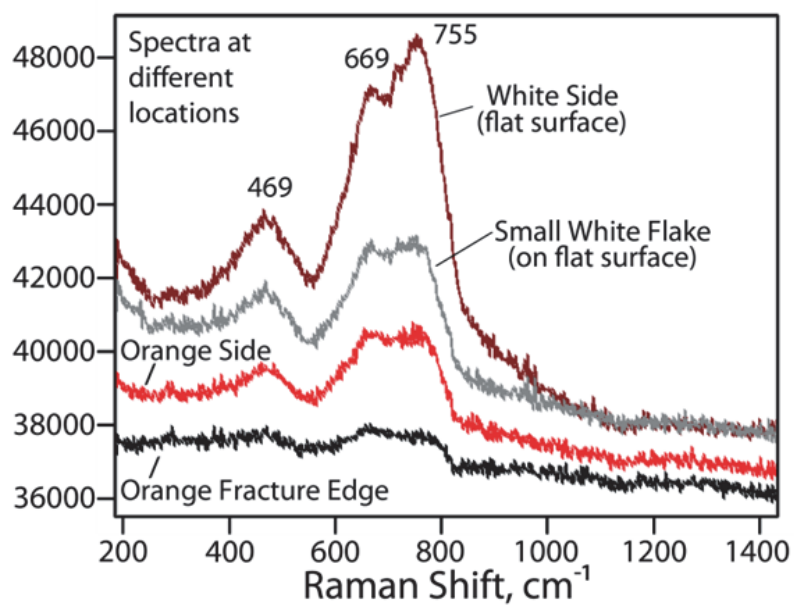

Figure 4.31. Micro-Raman spectroscopy of separated phases in $\mathrm{T} 2 \mathrm{G}-1-\mathrm{PbO}$ (same composition as $\mathrm{TPb}_{15}$ ).

The glass structure in the lead-tellurite and some of the structural variation glasses was further assessed by investigating the Raman spectra. Some representative Raman spectra are shown in Figure 4.32. From both parts of Figure 4.32 it can clearly be observed that adding $X \mathrm{Cl}_{20}$ to these glasses reduces the overall Raman intensity. Spectra were fitted as previously described, and the resultant area deconvolution is shown in Table 4.15 according to the bands assigned in Table 3.13.

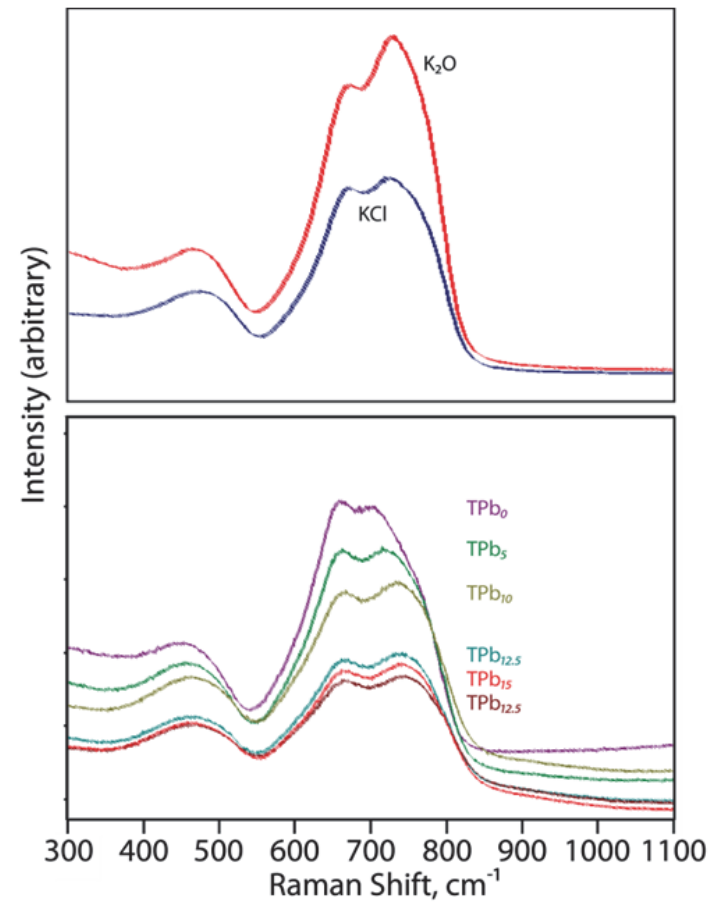

Figure 4.32. Raman spectra of $\mathrm{K}_{2} \mathrm{O}$ and $\mathrm{KCl}$ glasses (top) and some of the lead-tellurite glasses (bottom). 
Table 4.15. Fitted Raman data for lead-tellurite and some of the structural variation study glasses. The band names are listed in bold type. Note that the frequencies were fixed during the fitting and are shown at the top of each column of integrated intensities.

\begin{tabular}{llcccccc}
\hline & $\tilde{v}\left(\mathrm{~cm}^{-1}\right):$ & 432.7 & 476 & 596.5 & 648 & 710.9 & 769.7 \\
\hline $\mathrm{Name}$ & Alternate & $\mathbf{E} 1$ & $\mathbf{E 2}$ & $\mathbf{D}$ & $\mathbf{C}$ & $\mathbf{B}$ & $\mathbf{A}$ \\
\hline $\mathrm{TPb}_{0}$ & $\mathrm{~T} 2 \mathrm{G}-21$ & $3.84 \mathrm{E}+05$ & $2.40 \mathrm{E}+05$ & $3.73 \mathrm{E}+05$ & $1.48 \mathrm{E}+06$ & $2.49 \mathrm{E}+06$ & $6.82 \mathrm{E}+05$ \\
$\mathrm{P}-8$ & - & $6.27 \mathrm{E}+06$ & $6.21 \mathrm{E}+06$ & $9.10 \mathrm{E}+06$ & $3.40 \mathrm{E}+07$ & $6.03 \mathrm{E}+07$ & $1.55 \mathrm{E}+07$ \\
$\mathrm{TPb}_{5}$ & $\mathrm{~T} 2 \mathrm{G}-22$ & $3.49 \mathrm{E}+05$ & $3.53 \mathrm{E}+05$ & $3.27 \mathrm{E}+05$ & $1.22 \mathrm{E}+06$ & $2.14 \mathrm{E}+06$ & $1.04 \mathrm{E}+06$ \\
$\mathrm{TPb}_{10}$ & $\mathrm{~T} 2 \mathrm{G}-23$ & $2.50 \mathrm{E}+05$ & $4.60 \mathrm{E}+05$ & $2.78 \mathrm{E}+05$ & $8.59 \mathrm{E}+05$ & $1.77 \mathrm{E}+06$ & $1.06 \mathrm{E}+06$ \\
$\mathrm{P}-11$ & - & $1.33 \mathrm{E}+06$ & $1.90 \mathrm{E}+06$ & $1.15 \mathrm{E}+06$ & $4.87 \mathrm{E}+06$ & $1.01 \mathrm{E}+07$ & $6.36 \mathrm{E}+06$ \\
$\mathrm{TPb}_{12.5}$ & $\mathrm{~T} 2 \mathrm{G}-24$ & $1.99 \mathrm{E}+05$ & $4.02 \mathrm{E}+05$ & $1.98 \mathrm{E}+05$ & $5.86 \mathrm{E}+05$ & $1.43 \mathrm{E}+06$ & $7.83 \mathrm{E}+05$ \\
$\mathrm{TPb}_{14}$ & $\mathrm{~T} 2 \mathrm{G}-30$ & $3.07 \mathrm{E}+05$ & $9.46 \mathrm{E}+05$ & $5.80 \mathrm{E}+05$ & $1.46 \mathrm{E}+06$ & $3.41 \mathrm{E}+06$ & $2.11 \mathrm{E}+06$ \\
$\mathrm{TPb}_{14-\text { repeat }}$ & $\mathrm{T} 2 \mathrm{G}-30 \mathrm{~b}$ & $4.35 \mathrm{E}+04$ & $9.69 \mathrm{E}+04$ & $2.45 \mathrm{E}+04$ & $1.57 \mathrm{E}+05$ & $4.56 \mathrm{E}+05$ & $2.56 \mathrm{E}+05$ \\
$\mathrm{TPb}_{15-\text { powder }}$ & $\mathrm{T} 2 \mathrm{G}-1$ & $1.39 \mathrm{E}+05$ & $4.46 \mathrm{E}+05$ & $1.32 \mathrm{E}+05$ & $4.56 \mathrm{E}+05$ & $1.32 \mathrm{E}+06$ & $6.58 \mathrm{E}+05$ \\
$\mathrm{TPb}_{15-\text { white }}$ & $\mathrm{T} 2 \mathrm{G}-1$ & $8.03 \mathrm{E}+04$ & $1.11 \mathrm{E}+05$ & $3.01 \mathrm{E}+04$ & $2.60 \mathrm{E}+05$ & $4.84 \mathrm{E}+05$ & $4.45 \mathrm{E}+05$ \\
$\mathrm{TPb}_{17.5}$ & $\mathrm{~T} 2 \mathrm{G}-25$ & $2.16 \mathrm{E}+05$ & $4.47 \mathrm{E}+05$ & $1.80 \mathrm{E}+05$ & $5.02 \mathrm{E}+05$ & $1.52 \mathrm{E}+06$ & $7.30 \mathrm{E}+05$ \\
$\mathrm{TPb}_{22.5}$ & $\mathrm{~T} 2 \mathrm{G}-30$ & $9.58 \mathrm{E}+04$ & $2.91 \mathrm{E}+05$ & $1.15 \mathrm{E}+05$ & $4.53 \mathrm{E}+05$ & $1.20 \mathrm{E}+06$ & $6.84 \mathrm{E}+05$ \\
\hline $\mathrm{K}_{2} \mathrm{O}$ & $\mathrm{T} 2 \mathrm{G}-27 \mathrm{a}$ & $6.17 \mathrm{E}+05$ & $6.85 \mathrm{E}+05$ & $1.00 \mathrm{E}+06$ & $2.67 \mathrm{E}+06$ & $7.70 \mathrm{E}+06$ & $2.89 \mathrm{E}+06$ \\
$\mathrm{KCl}$ & $\mathrm{T} 2 \mathrm{G}-27 \mathrm{~b}$ & $6.35 \mathrm{E}+05$ & $6.42 \mathrm{E}+05$ & $4.85 \mathrm{E}+05$ & $1.93 \mathrm{E}+06$ & $4.57 \mathrm{E}+06$ & $1.74 \mathrm{E}+06$ \\
\hline$X \mathrm{O}_{20}$ & $\mathrm{~T} 2 \mathrm{G}-34$ & $5.71 \mathrm{E}+04$ & $7.92 \mathrm{E}+04$ & $1.09 \mathrm{E}+05$ & $3.08 \mathrm{E}+05$ & $7.55 \mathrm{E}+05$ & $3.02 \mathrm{E}+05$ \\
\hline
\end{tabular}

A progression of the $t b p / t p$ relative ratio, which indicates polymerization of the tellurite network, as determined from the area ratio $(\mathbf{C}+\mathbf{D}) /(\mathbf{A}+\mathbf{B})$ in the high frequency envelope, is shown in Figure 4.33a. The trend is a smooth decrease until about 15 mass $\%$, after which it remains nearly unchanged. Waste loadings $0,10,14$, and 15 mass $\%$ have two data points each, and the line is an average ratio of the two (Figure 4.33a). Noting that the 14 mass\% points are considerably different, it may be that the 14 mass $\%$ glass is on the verge of major structural change associated with devitrification, and slightly different cooling profiles produce different glass structures. It should be remembered that 15 mass $\% X_{20}$ represented the point at which phase separation began to be visibly apparent. One of the data points at 15 mass\% represents the Raman spectra taken on a powdered glass containing both phases, and the other is a fit of the white phase micro-Raman data. In contrast with the 14 mass $\%$ data, these 15 mass $\%$ ratios are remarkably similar to each other. At loadings higher than that inducing phase separation, it appears that the relative depolymerization of the tellurite network (as measured by $t b p / t p$ ) proceeds no further. From the micro-Raman and EDS on the phase-separated 15 mass $\% X \mathrm{Cl}_{20}$, the orange-red glassy phase is enriched in Te and has more tbp groups than the cloudy-whitish phase that is enriched in alkali, $\mathrm{Cl}$, and slightly in $\mathrm{Pb}$, and has more tp groups.

Figure $4.33 \mathrm{~b}$ shows a plot of the peak width as a function of waste loading for several of the Raman bands fitted with fixed frequencies. It is apparent that at least up to 15 mass $\% X \mathrm{Cl}$ loading and probably higher, the band width for $\mathbf{A}+\mathbf{B}$ bands assigned to $t p$ units increases, indicating increased local site disorder. By contrast, the band width of the $\mathbf{C}+\mathbf{D}$ bands assigned to tbp units do not change much with mixed chloride loading. This implies that the disorder with increasing additions of alkali, alkaline earth, lanthanide, and chloride ions manifests in the tp structural units, which makes sense since it is these 
depolymerized tellurite units that accommodate these ions in the glass structure. Since there are so many different waste ions to be incorporated, it seems reasonable that as the loading is increased, the number of different local $t p$ units would increase (i.e., the disorder increases). This effect seems to saturate near 15 mass $\%$ as also was seen with the area ratios in Figure 4.33a, and data in Figure 4.33b for the tp peaks $(\mathbf{A}+\mathbf{B})$ are also not inconsistent with a change in mechanism near 15 mass $\%$, but data are more scattered for the peak width.
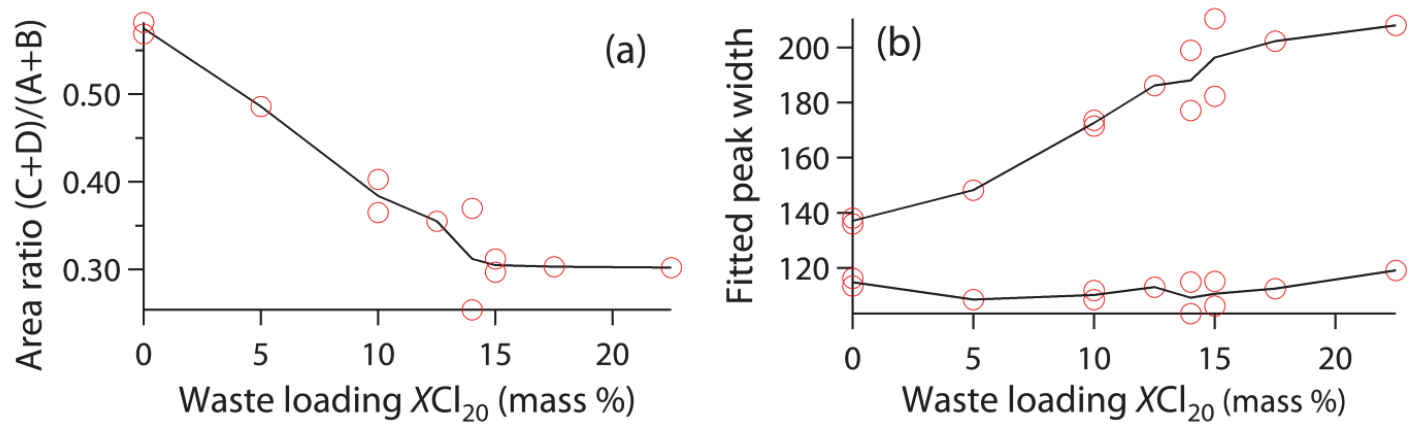

Figure 4.33. Quantitative Raman analysis for the lead-tellurite glasses. (a) $t b p / t p$ area ratio from Raman spectra as a function of waste loading. A lower $(\mathbf{C}+\mathbf{D}) /(\mathbf{A}+\mathbf{B})$ ratio represents a more depolymerized network. (b) Fitted peak width as a function of loading, indicating site disorder in the $t p$ part of the glass structure with increasing waste loading. In both figures, lines indicate averages where more than one data point (circle) is shown.

An examination of the effect of $\mathrm{Cl} / \mathrm{O}$ ratio for the structural variation glasses is shown in Table 4.16. From this data we can examine the effects of both $\mathrm{Cl}$ and cations. Comparing the $\mathrm{TPb}_{0}$ and $\mathrm{TPb}_{5}$ specimens, the addition of 5 mass $\% X_{20}$ results in a depolymerization of the network (lower area ratio), but it is not entirely clear whether this is due to the $\mathrm{Cl}$ or the cations in the waste or both. Comparing $\mathrm{TeO}_{2}-\mathrm{PbO}-\mathrm{XO}_{20}(\mathrm{~T} 2 \mathrm{G}-34)$ and $\mathrm{TeO}_{2}-\mathrm{PbO}-X \mathrm{Cl}_{20}\left(\mathrm{TPb}_{5}\right)$ gives an idea of the separate effects of the cations and chloride, since the amounts of $\mathrm{Te}$ and $\mathrm{Pb}$ relative to the waste cations is the same for both the oxide and the chloride waste. The data indicate that the chloride in $X \mathrm{Cl}_{20}$ actually repolymerizes the tellurite network compared to the $\mathrm{XO}_{20}$ glass with the same modifier cations, presumably by scavenging some of the alkali (or possibly alkaline earths and/or lanthanides), reducing the amount available for network breaking and formation of $t p$ units. Finally, comparison of the $\mathrm{K}_{2} \mathrm{O}$ and $\mathrm{KCl}$ glasses having the same relative amount of $\mathrm{K}$ compared to $\mathrm{Te}$ and $\mathrm{Pb}$ (see Table 3.10), indicates the same trend, i.e., that the chloride-containing glass is slightly more polymerized than the one without chloride when both contain the same relative amount of alkali to the main glass formers. This is in agreement with Tanaka et al., who observe a suppression of formation of the $t p$ units when $\mathrm{Cl}$ is substituted for $\mathrm{O}$ in $\mathrm{Li}-\mathrm{Te}-\mathrm{O}-\mathrm{Cl}$ glasses due to the strengthening of the Te- $\mathrm{O}_{\mathrm{ax}}$ bond since a smaller electronic charge is donated from $\mathrm{Te}-\mathrm{Cl}_{\mathrm{eq}}$ than

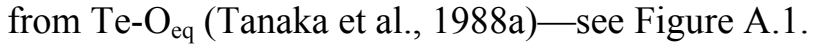

Table 4.16. Quantitative Raman analysis of some of the lead-tellurite and structural variation glasses.

\begin{tabular}{llcc}
\hline Sample ID & Glass System & $\mathrm{Cl} / \mathrm{O}$ & $(\mathrm{C}+\mathrm{D}) /(\mathrm{A}+\mathrm{B})$ \\
\hline T2G-21 $\left(\mathrm{TPb}_{0}\right)$ & $\mathrm{TeO}_{2}-\mathrm{PbO}\left(\mathrm{TPb}_{0}\right)$ & 0.000 & 0.58 \\
T2G-22 $\left(\mathrm{TPb}_{5}\right)$ & $\mathrm{TeO}_{2}-\mathrm{PbO}-X \mathrm{Cl}\left(\mathrm{TPb}_{5}\right)$ & 0.080 & 0.49 \\
T2G-34 $\left(\mathrm{XO}_{20}\right)$ & $\mathrm{TeO}_{2}-\mathrm{PbO}-X \mathrm{O}$ & 0.000 & 0.39 \\
T2G-29a & $\mathrm{TeO}_{2}-\mathrm{PbO}-\mathrm{K}_{2} \mathrm{O}$ & 0.000 & 0.35 \\
T2G-29b & $\mathrm{TeO}_{2}-\mathrm{PbO}-\mathrm{KCl}$ & 0.096 & 0.38 \\
\hline
\end{tabular}

\subsubsection{X-Ray Diffraction (XRD) Analysis}

The XRD results for the lead-tellurite glasses are presented in Figure 4.34 where the best-fitting phases were identified as those presented in Table 4.17. XRD analysis showed a small fraction of octahedral 
lead tellurite $\left(\mathrm{Pb}_{2} \mathrm{Te}_{3} \mathrm{O}_{7}\right)$ crystals in $\mathrm{TPb}_{0}$ observed with optical microscopy (Kulcu, 1987). $\mathrm{TPb}_{5}, \mathrm{TPb}_{10}$, $\mathrm{TPb}_{12.5}$, and $\mathrm{TPb}_{14}$ were determined to be amorphous whereas $\mathrm{TPb}_{15}, \mathrm{TPb}_{17.5}$, and $\mathrm{TPb}_{22.5}$ showed small degrees of crystallization. The glasses with $X \mathrm{Cl}_{20}$-loadings at $\geq 15$ mass $\%$ looked highly crystalline, so the small degree of crystallization was surprising considering their visual appearance. Later, with SEM, we verified that the observed color change was the result of nano-phase-separation of alkali chloride inclusions in the yellow-white phase (see Figure 4.36). At 15 mass\% loading, a small fraction of $\mathrm{PbO}$ was observed in the diffraction pattern. This $\mathrm{PbO}$ was not observed as a crystalline product in any of the other glasses. At $X \mathrm{Cl}_{20}$-loadings $>15$ mass $\%$, chloride phases were observed. At an $X \mathrm{Cl}_{20}$-loading of 17.5 mass\% $\left(\mathrm{TPb}_{17.5}\right), \mathrm{NaClO}_{3}, \mathrm{Ba}_{4} \mathrm{OCl}_{6}$, and $\mathrm{BaPrO}_{3}$ were the best-fitting patterns and at an $X \mathrm{Cl}_{20}$-loading of 22.5 mass $\%\left(\mathrm{TPb}_{22.5}\right), \mathrm{KCl}, \mathrm{NaCl}, \mathrm{CsPbCl}_{3}$, and $\mathrm{K}_{2} \mathrm{PrCl}_{5}$ were the best-fitting patterns. Since $\mathrm{CsPbCl}_{3}$ is the only one of these seven phases that contains the glass-forming components, this suggests that the waste-loading limit was achieved because the waste components were no longer stable in the glass network.

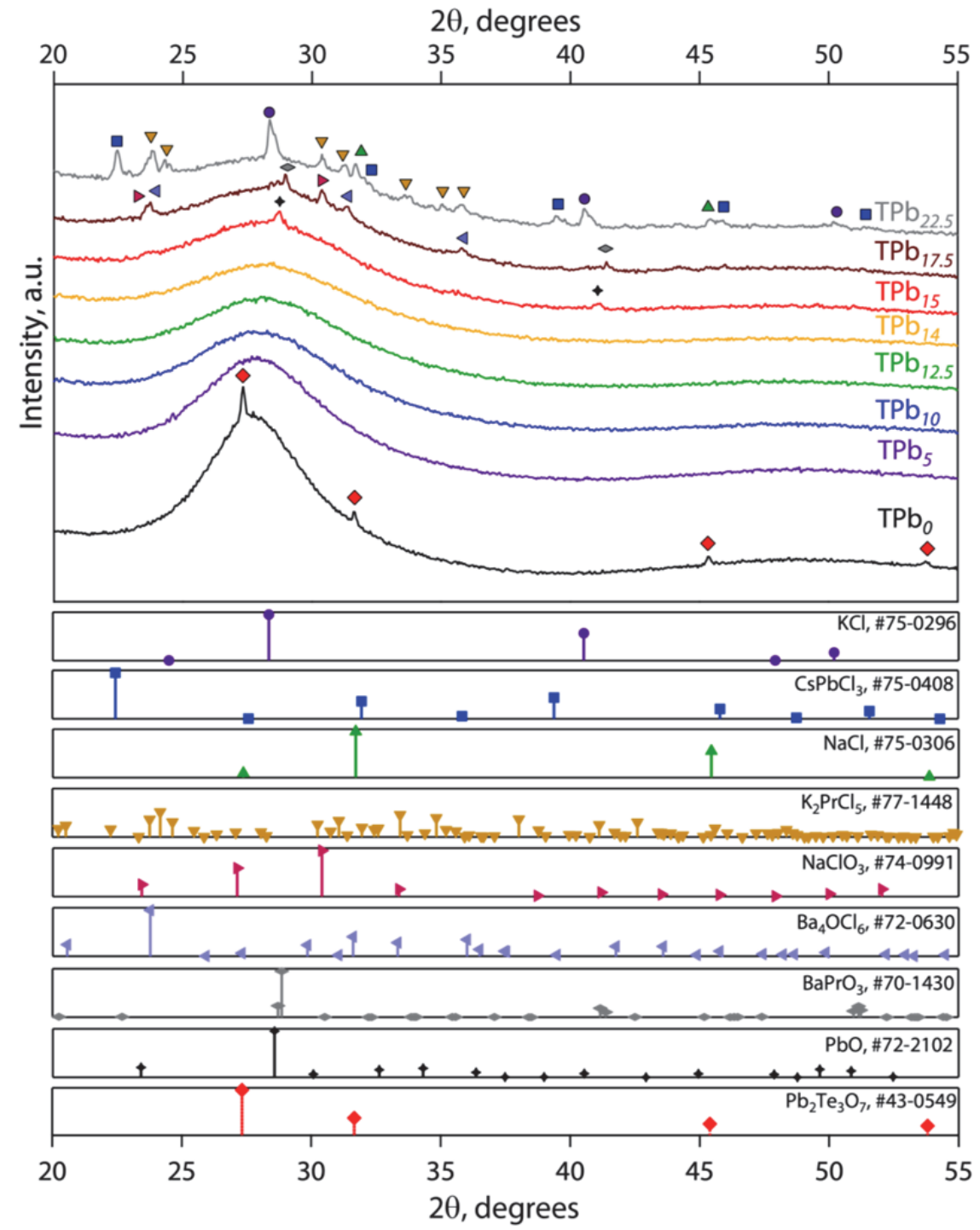

Figure 4.34. Summary of XRD results for lead-tellurite variation study glasses. The details of these phases can be found in Table 4.17 . 
Table 4.17. Summary of crystalline compounds identified in lead-tellurite glasses with XRD.

\begin{tabular}{lllll}
\hline Compound & PDF\# & ICSD\# & SG (SG\#) & Reference \\
\hline $\mathrm{KCl}$ & $75-0296$ & 28938 & $F m-3 m(225)$ & (Barrett and Wallace, 1954) \\
$\mathrm{NaCl}$ & $75-0306$ & 28948 & $F m-3 m(225)$ & (Barrett and Wallace, 1954) \\
$\mathrm{CsPCl}_{3}$ & $75-0408$ & 29067 & $P m-3 m(221)$ & (Moller, 1957) \\
$\mathrm{K}_{2} \mathrm{PrCl}_{5}$ & $77-1448$ & 48190 & $P n m a(62)$ & (Meyer et al., 1985) \\
$\mathrm{NaClO}_{3}$ & $74-0991$ & 26684 & $P 213(198)$ & (Zachariasen, 1929) \\
$\mathrm{Ba}_{4} \mathrm{OCl}_{6}$ & $72-0630$ & 16026 & $P 63 m c(186)$ & (Frit et al., 1970) \\
$\mathrm{BaPrO}_{3}$ & $70-1430$ & 2753 & $P b n m(62)$ & (Jacobson et al., 1972) \\
$\mathrm{PbO}$ & $72-2102$ & 20362 & $P b c n(60)$ & (Zaslavsky et al., 1950) \\
$\mathrm{Pb}_{2} \mathrm{Te}_{3} \mathrm{O}_{7}$ & $43-0549$ & N/A & $F m-3 m(225)$ & (Kulcu, 1987) \\
\hline
\end{tabular}

Background curves were fit to the XRD spectra in order to better distinguish fluctuations in the amorphous background and help with determining amorphous hump maxima. The fitted backgrounds from the XRD spectra are plotted in Figure 4.35a to accentuate minor differences in amorphous structure. From this plot, it is apparent that the amorphous structure of the $X \mathrm{Cl}_{20}$-loaded glasses is fairly consistent, although different from $\mathrm{TPb}_{0}$ without the $X \mathrm{Cl}_{20}$ addition. As seen in Figure 4.35b, the locations of the first and second broad amorphous hump maxima shift consistently with increasing $X \mathrm{Cl}_{20}$ loading, revealing a systematic shift in the amorphous structure as the waste components modify the glass network.
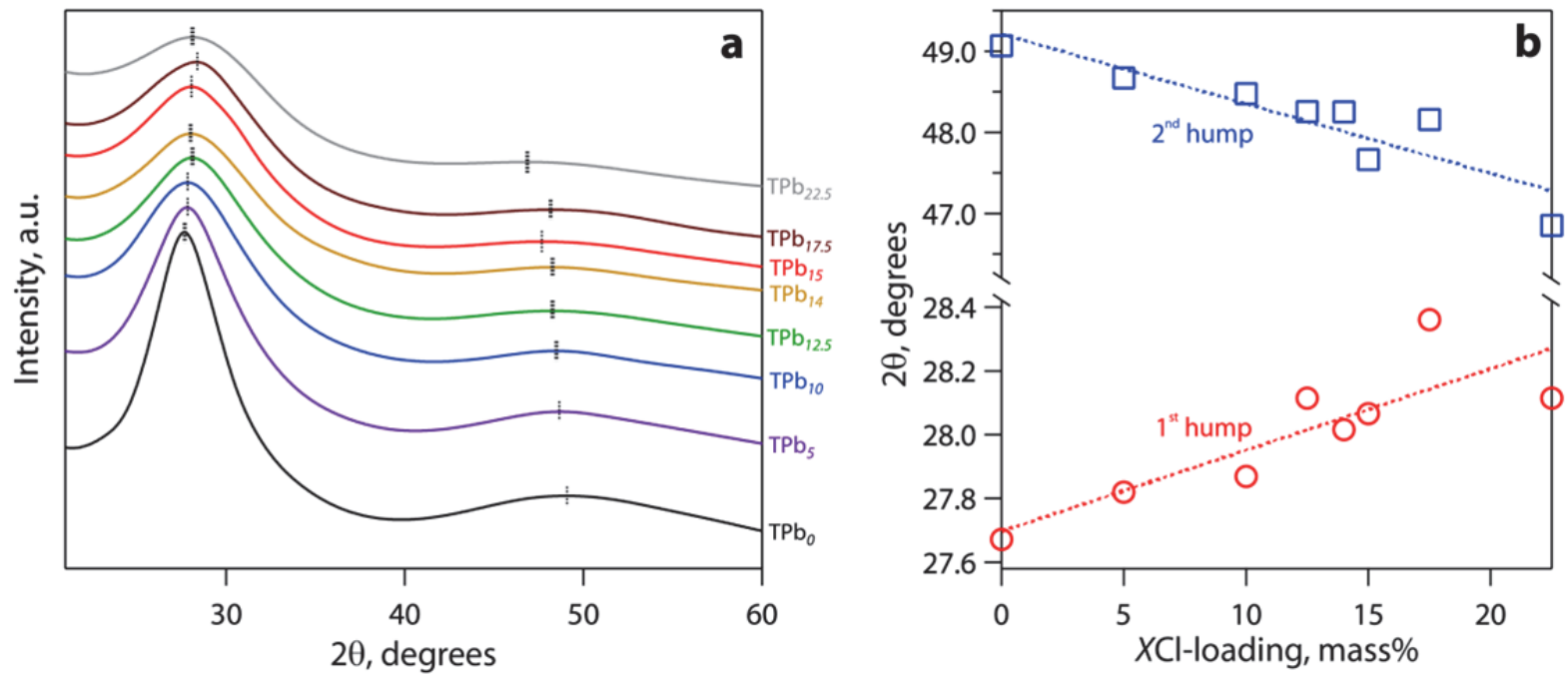

Figure 4.35. (a) Background fits on raw XRD data from the lead-tellurite glasses presented in Figure 4.34. (b) Amorphous hump maxima locations in 20 - locations denoted by vertical lines in (a) - along with linear data fits for informational purposes only.

\subsubsection{Scanning Electron Microscopy and Energy Dispersive Spectroscopy}

After observing the T2G-1-PbO glass in the SEM, it was apparent that the yellow-white phase was actually phase-separated from the bulk glass. This phase-separation was only observed in the top, yellowwhite portion of the glass (which cooled slowest during quenching) as dark spherical inclusions-see Figure 4.36. These dark inclusions had a much lower average atomic number than the surrounding matrix as evidenced by the backscattered imaging technique used to collect the micrographs where elements with a higher atomic number appear brighter than elements with a lower average atomic number. 
In the yellow-white phase, these inclusions ranged in size from $288 \pm 38 \mathrm{~nm}$ in diameter towards the top of the yellow-white phase to smaller droplets at $61 \pm 10 \mathrm{~nm}$ towards the bottom of the yellow-white phase, next to the glassy phase. Very few droplets were observed in the top $5 \mu \mathrm{m}$ of the yellow-white phase at the very top of the specimen and we believe that this is due to the fact that this surface was cooled by the air during quenching more rapidly than the glass just below the surface. Also the droplets that were found at the top of the specimen were very small $(<60 \mathrm{~nm})$.

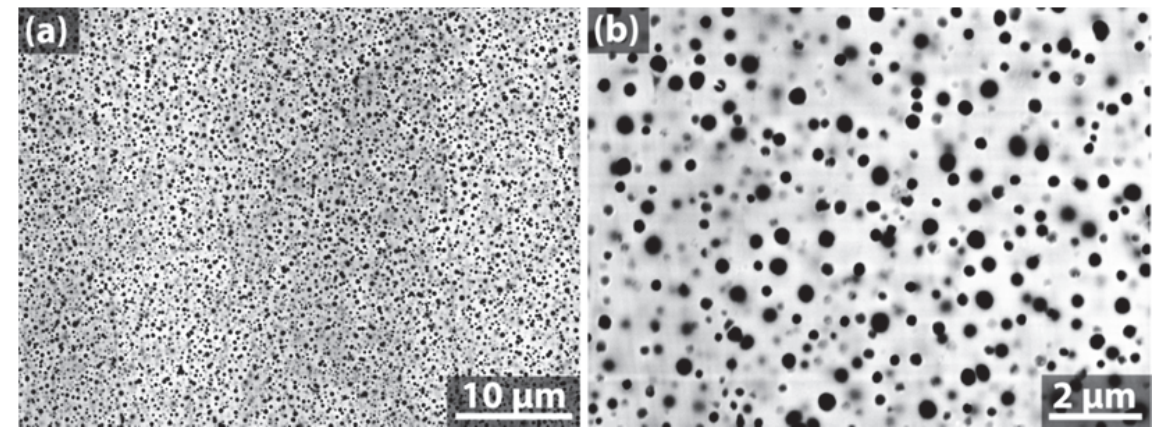

Figure 4.36. Scanning electron microscopy of yellow-white region in T2G-1-PbO showing nano-droplet phase-separation.

EDS area analysis was performed on several of these inclusions in different regions and the compositional results were compared with the results from a series of area scans of the surrounding matrix - see Figure 4.37. From Figure 4.37, it is apparent that the droplets show a higher concentration of the alkali chloride mixture, a higher tellurium concentration, and a lower lanthanum concentration than the surrounding matrix. These results suggest the following:

1) As the $X \mathrm{Cl}_{20}$-loading was increased in these glasses, some of the $X \mathrm{Cl}_{20}$ components were incorporated into the glass (e.g., $\mathrm{Ln}$ ) while others (e.g., $\mathrm{Na}, \mathrm{K}$, and $\mathrm{Cl}$ ) were excluded from the glassy phase in regions where the cooling rate was not sufficiently high.

2) As the $\mathrm{XCl}_{20}$-loading was increased, the alkali chlorides were not incorporated into the glass as well as the fraction of lanthanide salts.

3) The Ln fractions observed in both the glassy and inclusion phase were significantly higher in the phase-separated fraction of the specimen $(>20$ mass $\%)$ than in the as-batched glass $(\sim 8.3$ mass \%) - see Figure $4.37 \mathrm{~b}$. This suggests that the Ln fraction from the lower, glassy region of the specimen was pushed up into the upper phase-separated portion.
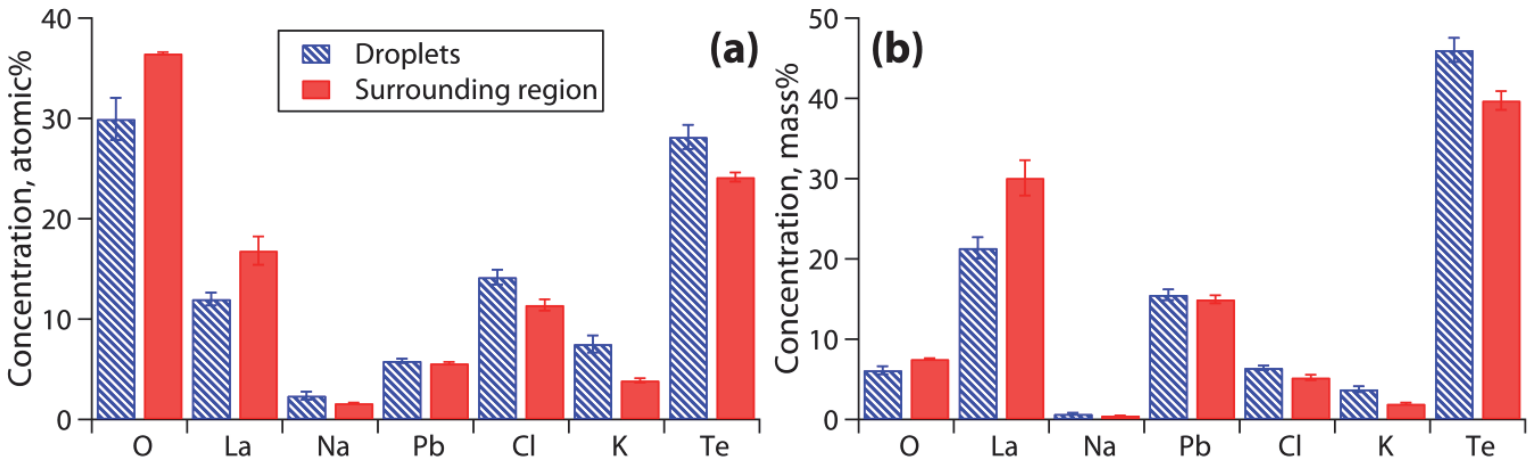

Figure 4.37. Energy dispersive spectroscopy on phase-separated region of $\mathrm{T} 2 \mathrm{G}-1-\mathrm{PbO}$ where the average composition of the dark droplets in Figure 4.36 is compared to that of the surrounding lighter matrix. The data is presented in both (a) atomic\% and (b) mass \%. It should be noted that that Li cannot be detected with this technique and is likely present in the droplets along with the other alkali cations. We expect that that the compositional values for the droplets are slightly skewed by the bulk matrix just below the droplets due to electron beam penetration into and interaction with the subsurface matrix. 
These results reveal the real reason why the yellow-white phase on the top of T2G-1-PbO is opaque, which is that these phase-separated droplets of alkali halide salt are so small that they cause lightscattering centers, preventing the light to transmit through the specimen in these regions. It is interesting that the alkali chloride phase(s) did not show up on the XRD spectrum for this glass (Figure 4.34), even though they were present in such large quantities considering that these inclusions were found on the top $\sim 40 \%$ of the glass in a fairly high density ( $>15 \%$ by volume). We presume that this implies that the alkali halide droplets are amorphous in structure although this has not yet been confirmed.

Figure 4.38 shows the salt phase separation in the higher salt loaded glasses. In the $22.5 \%$ glass, large regions were found where salt droplets (perimeter of $22.5 \%$ micrograph) coalesced into large pools of molten salt (middle of $22.5 \%$ micrograph). The surrounding region can be seen as devoid of droplets but then the droplets appear very small before growing larger in diameter further away from the pool. This phenomenon is typical of crystal growth on cooling depleting the surrounding area of the elements needed for crystal growth. The $30 \%$ glass did not have these pools but rather a very high density of droplets all throughout the glass, with varying compositions. The droplets appeared both darker and lighter than the bulk glass. The bright droplets had a higher concentration of $\mathrm{Pb}, \mathrm{La}$, and $\mathrm{Cl}$ where the darker phase was richer in $\mathrm{K}$ and $\mathrm{Cl}$ (compared to the surrounding glass).
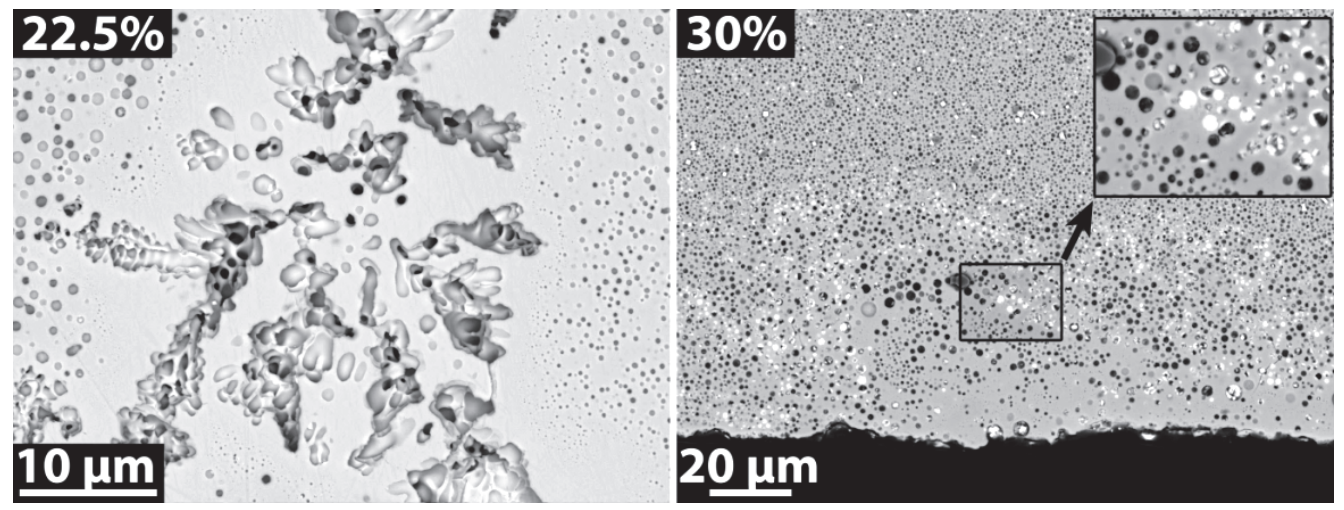

Figure 4.38. $\mathrm{SEM}$ micrographs of $\mathrm{TPb}_{22.5}$ (left) showing semi-continuous salt phase formation and $\mathrm{TPb}_{30}$ (right) showing high density droplets.

\subsubsection{Transmission Electron Microscopy}

Conventional TEM and analytical STEM techniques were performed on the glasses seemingly devoid of phase separation (10 and 14 mass\%) to assess whether phase separation was nucleating at lower salt concentrations. At 10 mass $\% \mathrm{XCl}_{20}$, the glass was observed to be amorphous (diffraction/BF) with no appearance of phase separation (Figure 4.39). Holding the beam on the specimen for extended periods of time induced only slight melting near the edges. At 14 mass $\% X \mathrm{Cl}_{20}, 10-20 \mathrm{~nm}$ rounded contrast features were observed in the glass. Diffraction of these regions suggested that the second phase was not crystalline, but the decreased intensity resulting from use of the $20-\mu \mathrm{m}$ diameter aperture (to inhibit beam induced phase separation) made it difficult to collect large areas with any appreciable intensity. If the phase-separated regions were indeed poorly crystalline, a larger concentration would be needed to observe diffraction in TEM. When a 50- $\mu$ m diameter condenser aperture was inserted, beam-induced phase separation was observed as the particles grew slightly (from $\sim 10$ to $\sim 18 \mathrm{~nm}$ ) and increased in concentration. High resolution imaging and diffraction suggested that there was some crystallinity in these phase-separated regions induced by beam damage. Thus it was found that nano-scale phase separation had, in fact, begun with 14 mass $\% X_{20}$, despite the uniform appearance in visible light and with SEM. 

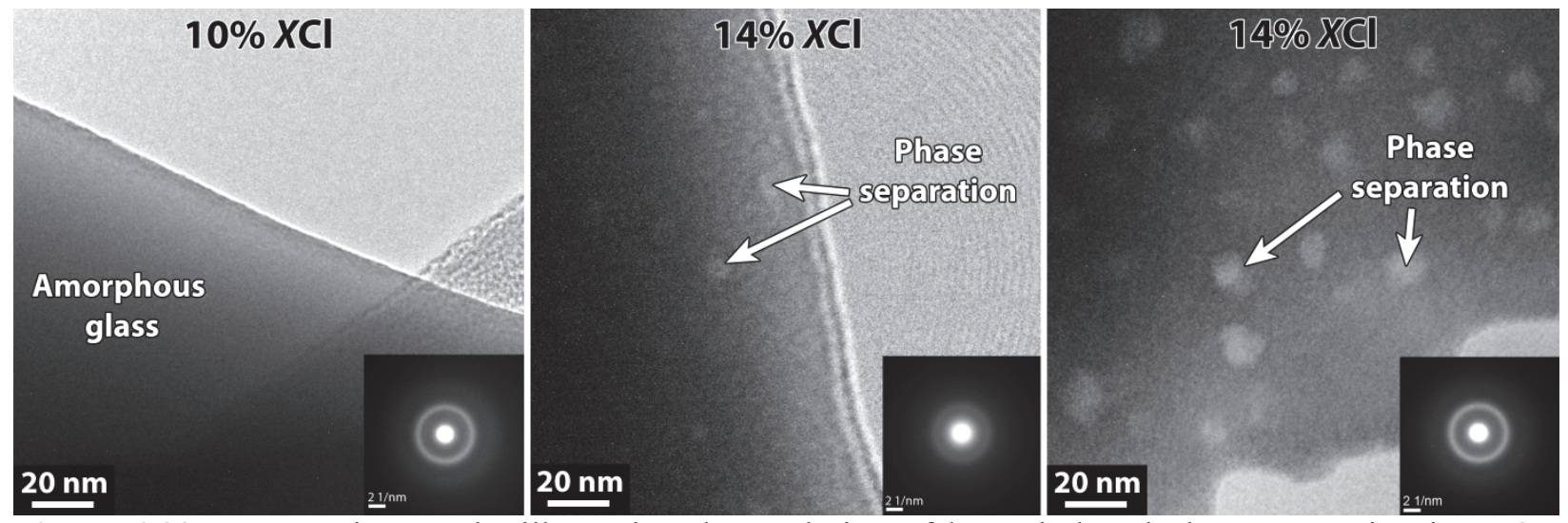

Figure 4.39. TEM micrographs illustrating the evolution of beam-induced phase separation in $\mathrm{TeO}_{2}$ $\mathrm{PbO}-\mathrm{XCl}_{20}$ glasses $(10 \%, 14 \%)$. The 10 mass $\% \mathrm{XCl}_{20}$ specimen is completely amorphous. The 14 mass $\% \mathrm{XCl}_{20}$ has phase separated regions $\sim 10 \mathrm{~nm}$ in diameter. Using a larger condenser aperture to image the specimen, beam-induced phase separation occurs (higher density of phase-separated regions which are slightly larger, $\sim 18 \mathrm{~nm}$ ).

When the $X \mathrm{Cl}_{20}$ loading increased above the phase separation region, crystalline phases became evident (Figure 4.40). At $17.5 \% \mathrm{XCl}_{20}$-loading, a high concentration of large $(30-60 \mathrm{~nm})$ diameter phase separation was observed as crystalline material. At higher $X \mathrm{Cl}_{20}$-loading $(22.5 \%)$, the phase separation was observed as 300-400 nm diameter pockets with a lower concentration than the $17.5 \%$ loading. With EDS analysis, the composition of the amorphous regions and the phase separated regions with varying contrast were examined. The darker of the four particles were observed to be high in $\mathrm{Pb}$ and contained $\mathrm{Cs}$. The lighter of the four phase separated regions contained no $\mathrm{Cs}$ and had lower $\mathrm{Pb}$ but higher $\mathrm{Cl}$ content than the darker regions. Due to charging and the shape of the glass particles, selected area diffraction was not attempted on these regions.

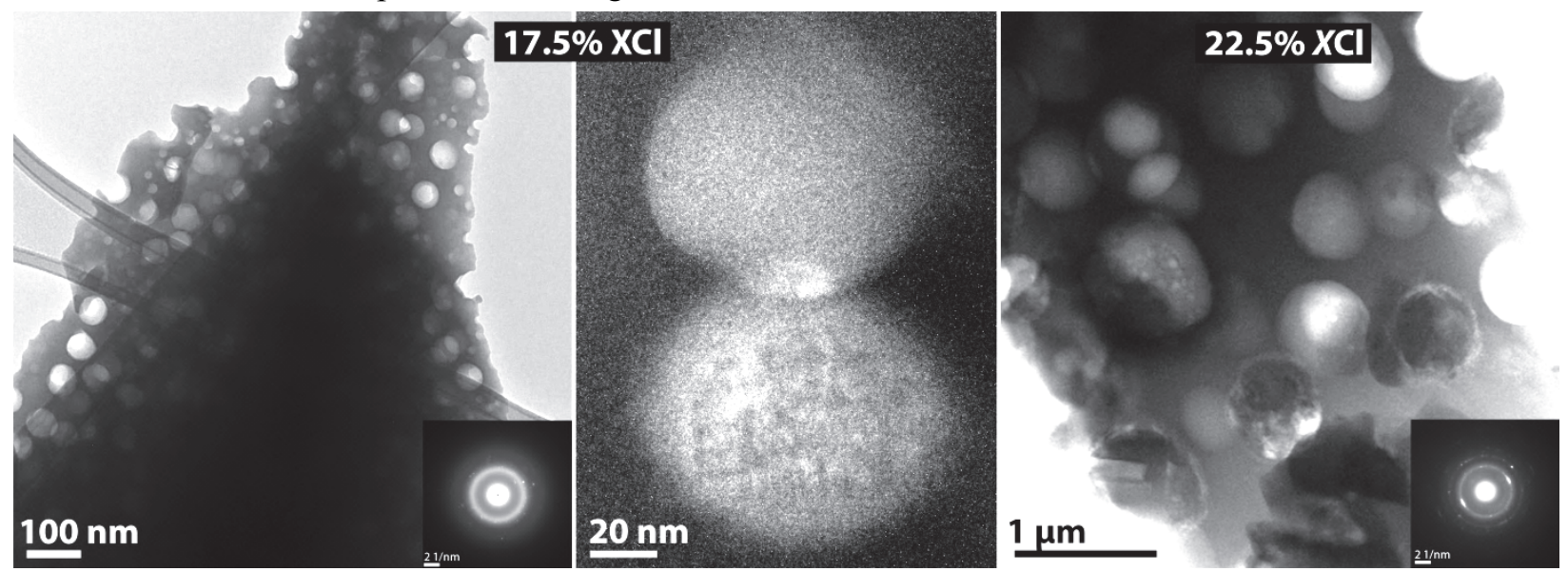

Figure 4.40. TEM micrographs showing phase separation and crystallization in $\mathrm{TeO}_{2}-\mathrm{PbO}-X \mathrm{Cl}$ glasses $(17,22.5 \%)$. At $17.5 \% \mathrm{XCl}_{20}$ loading, full phase separation is observed, with phase separation on the order of $30-60 \mathrm{~nm}$ in diameter. At $22.5 \% \mathrm{XCl}_{20}$ loading, the phase separation is observed to be on the order of 300-400 $\mathrm{nm}$ in diameter.

\subsubsection{Thermogravimetric Analysis}

Thermogravimetric analysis (TGA) of the lead-tellurite glasses showed that a small mass loss occurred during heating up to $\sim 600{ }^{\circ} \mathrm{C}\left(<1\right.$ mass\%) with an increasing mass loss at $T>600{ }^{\circ} \mathrm{C}(<6$ mass\%) where the glasses began to melt (Figure 4.41a). It is worth noting that the cumulative mass loss up to the heat- 
treatment temperature of $735^{\circ} \mathrm{C}$ increased in a consistent manner up to 14 mass $\% X \mathrm{Cl}_{20}$-loading, where it then plateaued (Figure 4.41b). This suggests that the $\mathrm{XCl}_{20}$ components, in addition to the maximum waste-loading (14 mass\%), are probably retained in the glass as inclusions during cooling from liquidliquid phase separation, as evidenced by SEM analysis (Figure 4.36), and not evolved.
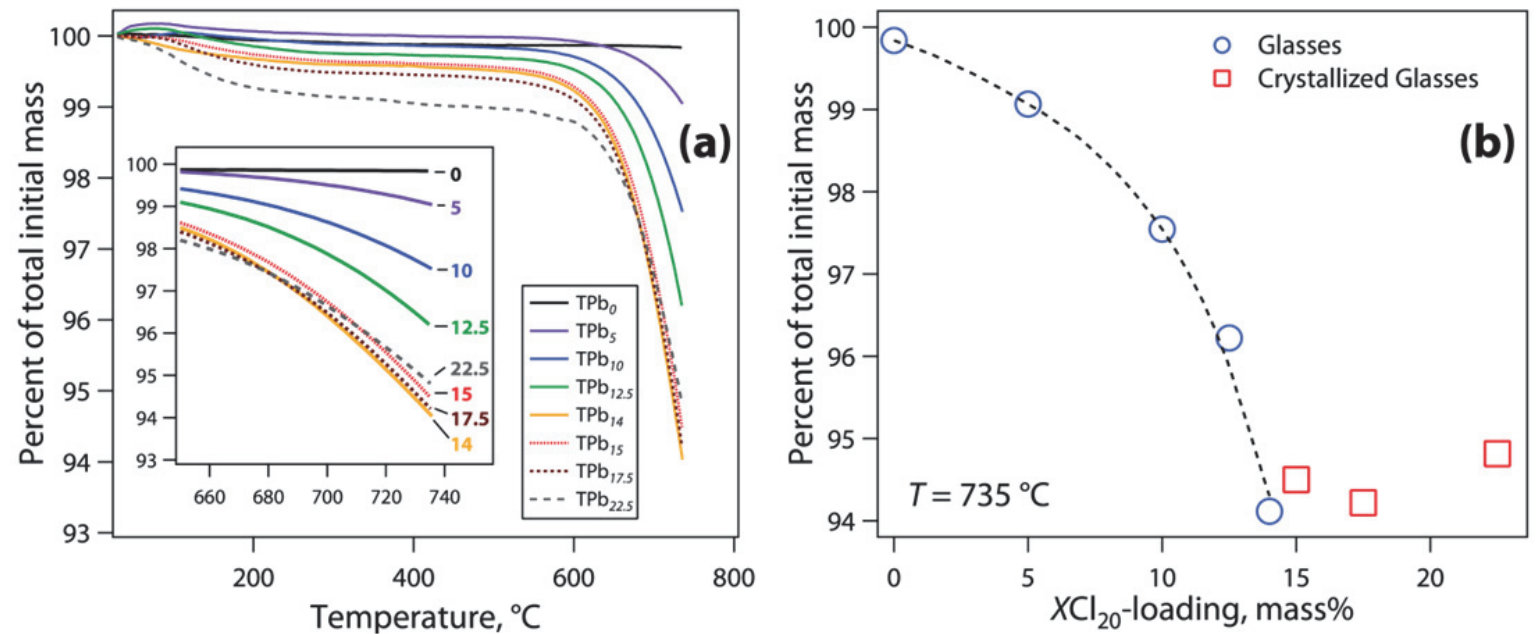

Figure 4.41. Summary of the TGA results for the lead-tellurite glasses. Figure (a) shows the thermogravimetric loss as a function of temperature; the inset shows a magnified view of the hightemperature end of the plot. (b) Cumulative mass retention at $735^{\circ} \mathrm{C}$ for all of the glasses.

The DSC results on the lead-tellurite glasses were interesting. We had difficulty setting up the instrument with a good baseline, especially at temperatures above $500{ }^{\circ} \mathrm{C}$. Thus, the data collected in that temperature range was not displayed in Figure 4.42a. Glass $\mathrm{TPb}_{0}$ had a distinct glass transition temperature $\left(T_{\mathrm{g}}\right)$ and two distinct exothermic events denoted by upward peaks of crystallization, $T_{\mathrm{c}(1)}$ at the lower temperature and $T_{\mathrm{c}(2)}$ at the higher temperature.

Table 4.18. Summary of the DSC results for the lead-tellurite glasses. Variable headings are defined in the paragraph above.

\begin{tabular}{llllll}
\hline $\mathrm{Glass}_{\mathrm{ID}}$ & Alternative ID & $T_{\mathrm{g}},{ }^{\circ} \mathrm{C}$ & $T_{\mathrm{c}(1)},{ }^{\circ} \mathrm{C}$ & $T_{\mathrm{c}(2)},{ }^{\circ} \mathrm{C}$ & $T_{\mathrm{M}},{ }^{\circ} \mathrm{C}$ \\
\hline $\mathrm{TPb}_{0}$ & $\mathrm{~T} 2 \mathrm{G}-21$ & 263.6 & 308.9 & 355.9 & 527.7 \\
$\mathrm{TPb}_{5}$ & $\mathrm{~T} 2 \mathrm{G}-22$ & 278.5 & - & 383.2 & - \\
$\mathrm{TPb}_{10}$ & $\mathrm{~T} 2 \mathrm{G}-23$ & 258.6 & - & 382.5 & - \\
$\mathrm{TPb}_{12.5}$ & $\mathrm{~T} 2 \mathrm{G}-24$ & 246.1 & 348.6 & 379.3 & - \\
$\mathrm{TPb}_{14}$ & $\mathrm{~T} 2 \mathrm{G}-31$ & 220.3 & 267.7 & 397.1 & - \\
$\mathrm{TPb}_{15}$ & $\mathrm{~T} 2 \mathrm{G}-26$ & 244.2 & 290.5 & 381.9 & - \\
$\mathrm{TPb}_{17.5}$ & $\mathrm{~T} 2 \mathrm{G}-25$ & 228.0 & 283.5 & 373.1 & - \\
$\mathrm{TPb}_{22.5}$ & $\mathrm{~T} 2 \mathrm{G}-30$ & 220.7 & 292.1 & 373.7 & - \\
\hline
\end{tabular}



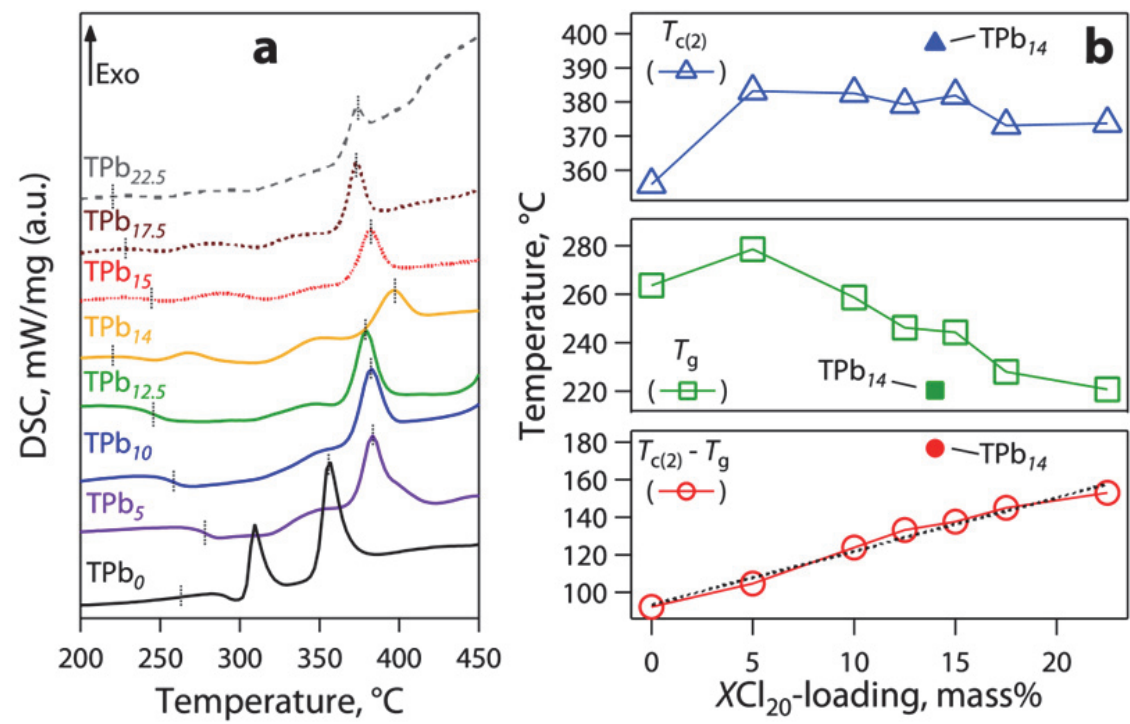

Figure 4.42. DSC results for the lead-tellurite glasses. Figure (a) shows the heat-flow curves as a function of temperature where the calculated $T_{\mathrm{g}}$ and $T_{\mathrm{c}(2)}$ are marked with vertical lines. Figure (b), plotted from the data in Table 4.18, shows the trends for $T_{\mathrm{c}(2)}\left(\right.$ top), $T_{\mathrm{g}}$ (middle), and $T_{\mathrm{c}(2)}-T_{\mathrm{g}}$ (bottom); $\mathrm{TPb}_{14}$ did not fit well on any of the plots so it is denoted by solid symbols on each trend.

As the $X \mathrm{Cl}_{20}$ was added to the $\mathrm{TPb}_{0}$ composition, the $T_{\mathrm{g}}$ shifted (both to higher and lower temperatures) the $T_{\mathrm{c}(1)}$ peak became buried in the noise, and the distance between the $T_{\mathrm{g}}$ and $T_{\mathrm{c}(2)}$ became larger and larger (Figure 4.42b), suggesting a greater glass formation ability—see Equation (9).

These data revealed a few things that were occurring in the glasses during heating:

1) The thermogravimetric mass loss during heating was very low at $T \leq 600^{\circ} \mathrm{C}(<1$ mass $\%)$ but increased at $T>600{ }^{\circ} \mathrm{C}(\leq 6$ mass $\%)$ where the glasses melted. Since all of these glasses were melted at the same temperature of $700{ }^{\circ} \mathrm{C}$, which is not likely to be the ideal temperature for all of them, this volatility could be reduced by melting the glasses at their ideal melting temperatures.

2) The total mass loss as a function of $X_{C_{20}}$-loading increased to the glass that had the maximum loading without phase separation $\left(\mathrm{TPb}_{14}\right)$ where it then plateaued. This suggests that the glass was stabilized by the added waste components at a waste loading above 14 mass $\% X_{C 2}$-loading.

3) Glassy phase(s) present seem to be stabilized by greater additions of the $X \mathrm{Cl}_{20}$ components according to Figure $4.42 \mathrm{~b}$ as evidenced by a greater $K_{\mathrm{gl}}$ as $X \mathrm{Cl}_{20}$-loading was increased. However, it is difficult to feel confident with this assessment after considering the phaseseparation observed with the SEM in the glasses with $\geq 15$ mass $\% X \mathrm{Cl}_{20}$-loading (Figure 4.36).

\subsubsection{Nuclear Magnetic Resonance}

Spectra of ${ }^{125} \mathrm{Te}$ NMR are often difficult to interpret, since ${ }^{125}$ Te is a spin $I=1 / 2$ nucleus with a large gyromagnetic ratio $\left(-8.5 \times 10^{-7} \mathrm{rad} /(\mathrm{T} \cdot \mathrm{s})\right)$ and a long spin-lattice relaxation time, resulting in a large chemical shift range of several thousands of ppm (Edwards et al., 2011). The relaxation time can be shortened if $\mathrm{Fe}_{2} \mathrm{O}_{3}$ is added to the samples, but this was not done for the current study (Sakida et al., 1999a; Sakida et al., 1999c). Experimental data was simulated to extract the isotropic chemical shift $\left(\delta_{\text {iso }}\right)$, chemical shift anisotropy $(\Delta \delta)$, and asymmetry parameter $(\eta)$. Disorder on the various NMR parameters was simulated to match the experimental data. It was found that providing $\pm 100 \mathrm{ppm}$ Gaussian disorder in $\delta_{\text {iso }}$ provided the necessary smoothing to match the experimental data, while providing disorder in $\Delta \delta( \pm 100 \mathrm{ppm})$ or $\eta( \pm 0.1)$ had little effect. All subsequent simulations were performed with this $\delta_{\text {iso }}$ disordering. All spectra could be fit with two Te sites, while one was insufficient and three did not significantly improve the fits. Figure 4.43 shows an example of experimental data, 
simulation, and deconvolution. A summary of all the NMR data collected for the $\mathrm{TeO}_{2}-\mathrm{PbO} X \mathrm{Cl}_{20}$ series is shown in Figure 4.44.

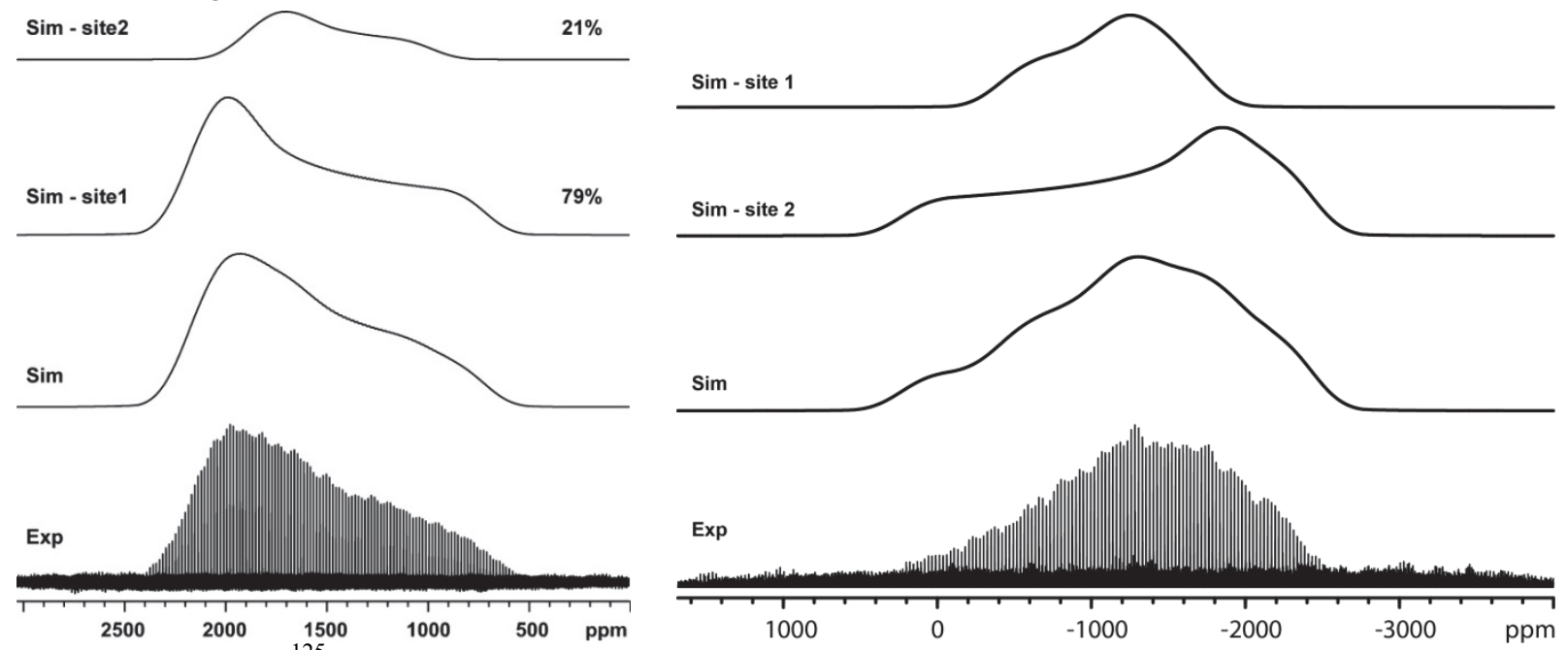

Figure 4.43. (left) ${ }^{125} \mathrm{Te} \mathrm{NMR}$ for $10 \% \mathrm{XCl}_{20}$ glass showing experiment (Exp), simulation (Sim), and site deconvolution. (right) ${ }^{207} \mathrm{~Pb} \mathrm{NMR}$ results for $15 \% \mathrm{XCl}_{20}$ glass.

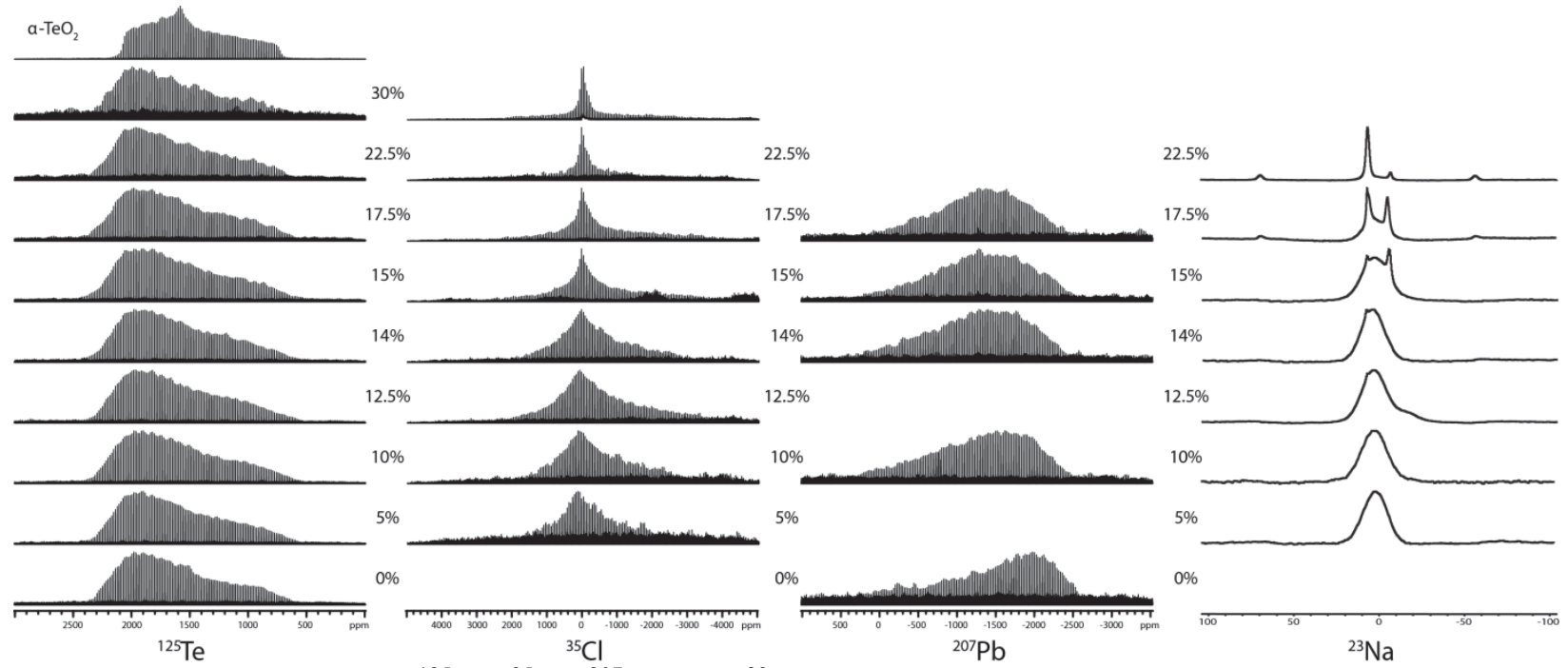

Figure 4.44. Summary of ${ }^{125} \mathrm{Te},{ }^{35} \mathrm{Cl},{ }^{207} \mathrm{~Pb}$, and ${ }^{23} \mathrm{Na} \mathrm{NMR}$ as a function of waste loading. Spectra are aligned by glasses and the gaps show were data was not collected.

The ${ }^{125} \mathrm{Te}$ fit results of the constant $\mathrm{Te} / \mathrm{Pb}$ ratio suggest that Te site distribution does not change with the addition of $X \mathrm{Cl}_{20}$ (see Table 4.19). The fit resulted in one Te site with larger chemical shift (mean 1642 ppm) having the greater area fraction (mean 78.7\%), herein assigned to $\mathrm{TeO}_{4}$. A second site with the smaller chemical shift (mean $1558 \mathrm{ppm}$ ) and area fraction (21.3\%) was assigned to $\mathrm{TeO}_{3}$.

For $\mathrm{TPb}_{0}$, the glass without chlorides, the area fractions were $78 \%\left(\mathrm{TeO}_{4}\right)$ and $22 \%\left(\mathrm{TeO}_{3}\right)$; these results are quite similar to results of Sakida et al. (1999b) for glasses in the range of $\mathrm{PbO}$ comparable to $\mathrm{TPb}_{0}$. However, the $\delta_{\text {iso }}$ and $\Delta \delta$ from our fits contradict the assessment of Sakida et al. In fitting the static ${ }^{125} \mathrm{Te}$ NMR data, Sakida et al. (1) fixed $\Delta \delta(-1285 \mathrm{ppm})$ and $\eta(0.74)$ based on pure $\mathrm{TeO}_{2}$ glass and assigned it to $\mathrm{TeO}_{4}$ tbp units, then (2) fit the second site $\left(\mathrm{TeO}_{3} t p\right.$ units) at the lowest $\mathrm{PbO}$ concentration and fixed $\Delta \delta$ $(-805 \mathrm{ppm})$ and $\eta(0.39)$ for all other glasses in the PbO series. The only adjustable parameters, then, 
became $\delta_{\text {iso }}$ and the fraction of sites. Their resulting $\delta_{\text {iso }}$ for the $\mathrm{TPb}_{0}$-equivalent glass was $\delta_{\text {iso }}\left(\mathrm{TeO}_{4}\right)$ $\sim 1577 \mathrm{ppm}$ and $\delta_{\text {iso }}\left(\mathrm{TeO}_{3}\right) \sim 1870 \mathrm{ppm}$. By contrast, in our fit, the lower chemical shift species with smaller area fraction was assigned to $\mathrm{TeO}_{3}$, so the assignments by area fractions were similar to Sakida et al. but the higher versus lower chemical shifts are reversed.

Table 4.19. ${ }^{125}$ Te static NMR fit results.

\begin{tabular}{|c|c|c|c|c|c|c|c|c|}
\hline \multirow{2}{*}{$\begin{array}{c}X C l \\
\text { Mass\% }\end{array}$} & \multicolumn{4}{|c|}{ Site $1\left(\mathrm{TeO}_{4}\right)$} & \multicolumn{4}{|c|}{ Site $2\left(\mathrm{TeO}_{3}\right)$} \\
\hline & $\delta_{\text {iso }}$ & $\Delta \delta$ & $\eta$ & Area\% & $\delta_{\text {iso }}$ & $\Delta \delta$ & $\eta$ & Area\% \\
\hline 0 & 1649 & -1364 & 0.217 & 78.2 & 1603 & -697 & 0.330 & 21.8 \\
\hline 5 & 1647 & -1370 & 0.282 & 79.1 & 1540 & -843 & 0.406 & 20.9 \\
\hline 10 & 1640 & -1387 & 0.259 & 78.6 & 1540 & -847 & 0.428 & 21.4 \\
\hline 12.5 & 1638 & -1418 & 0.270 & 77.8 & 1569 & -798 & 0.670 & 22.2 \\
\hline 14 & 1641 & -1425 & 0.263 & 77.5 & 1549 & -804 & 0.426 & 22.5 \\
\hline 15 & 1641 & -1384 & 0.280 & 79.6 & 1556 & -829 & 0.443 & 20.4 \\
\hline 17.5 & 1636 & -1423 & 0.265 & 80.8 & 1538 & -854 & 0.294 & 19.2 \\
\hline 22.5 & 1643 & -1441 & 0.215 & 77.9 & 1568 & -816 & 0.444 & 22.1 \\
\hline Mean & 1642 & -1401 & 0.256 & 78.7 & 1558 & -812 & 0.430 & 21.3 \\
\hline Std. Dev. & 4 & 29 & 0.026 & 1.1 & 22 & 51 & 0.112 & 1.1 \\
\hline 14 (high $\mathrm{Pb}$ ) & 1666 & -1383 & 0.183 & 71.8 & 1663 & -792 & 0.446 & 28.2 \\
\hline $5(X \mathrm{O})$ & 1650 & -1369 & 0.257 & 80.0 & 1599 & -714 & 0.443 & 20.0 \\
\hline
\end{tabular}

These discrepancies between our results and that of Sakida et al. (Sakida et al., 1999b) warrant further comment. Unlike those in Sakida et al., the methods used in the current study did not fix $\Delta \delta$ or $\eta$ for either of the Te sites but rather allowed all parameters to vary. In a different study, Sakida et al. (Sakida et al., 1999a) also modeled a large number of Te-containing crystals, and assigned the Te structure based on a diagram of $\Delta \delta$ versus $\eta$, later applying this to glass as well (Sakida et al., 1999c). Recently, Holland et al. have observed that Sakida's model did not work for their $\mathrm{Na}_{2} \mathrm{O}-\mathrm{TeO}_{2}$ crystallized glasses studied with ${ }^{23} \mathrm{Na}$ and ${ }^{125} \mathrm{Te} \mathrm{NMR}$, suggesting that caution is advised in comparing glass spectra to crystalline line shapes and inferring local structures (Holland et al., 2005). Likewise, our data for the $\mathrm{TeO}_{4}$ site $(|\Delta \delta|=$ $1401, \eta=0.26$ ) does not fit in one of Sakida's regions, but rather near some of the data from Holland et al.; on the other hand, our $\mathrm{TeO}_{3}$ site $(|\Delta \delta|=811, \eta=0.43)$ does fit in Sakida's "terminal $\mathrm{TeO}_{3}$ " region similar to other glasses (Sakida et al., 1999c).

The lack of change of the Te site distribution with increasing metal chloride addition contradicts commonly held assumptions about the effects of $\mathrm{M}^{+}, \mathrm{M}^{2+}$ and $\mathrm{M}^{3+}$ cations on the structure of $\mathrm{TeO}_{2}$ glasses in the following way (Heo et al., 1992; Sakida et al., 1999a; Sakida et al., 2001, 1999c; Sekiya et al., 1994b; Sekiya et al., 1992; Sekiya et al., 1995). In previous FTIR, Raman, and NMR studies where $\mathrm{M}^{+}$, $\mathrm{M}^{2+}$ and $\mathrm{M}^{3+}$ oxides are added to $\mathrm{TeO}_{2}$, increasing amounts of these oxides added to $\mathrm{TeO}_{2}$ glass are found to increase the $\mathrm{TeO}_{3}$ fraction of sites at the expense of $\mathrm{TeO}_{4}$ sites. In the case of the $\mathrm{TeO}_{2}-\mathrm{PbO}-X \mathrm{Cl}$ glasses, on the other hand, the Te site distribution is not changed by the addition or subtraction of cations and anions.

To test whether the Te:Pb ratio was controlling the Te speciation, a new "high Pb" glass (TG12-02) with a $\mathrm{Te}: \mathrm{Pb}$ ratio of 3.0 was prepared and analyzed. The ${ }^{125} \mathrm{Te}$ fitted spectrum showed that the Te speciation changed slightly (but measurably) from other samples, with a noticeably higher $\mathrm{TeO}_{3}$ fraction $(28.2 \%$ for high $\mathrm{Pb}$ versus $21.3 \%$ average for other samples). This glass showed that $\Delta \delta$ was within the range of the other glasses, but $\eta, \delta_{\text {iso }}$, and area fractions were clearly different. This sample should be compared directly with the $14 \% X \mathrm{Cl}_{20}$ glass. Additionally, the all-oxide $X \mathrm{O}$ glass, which should be compared with the $5 \% \mathrm{XCl}_{20}$ glass, appears distinguishable from the chloride glasses, with site $2\left(\mathrm{TeO}_{3}\right) \Delta \delta$ and $\delta_{\text {iso }}$ being

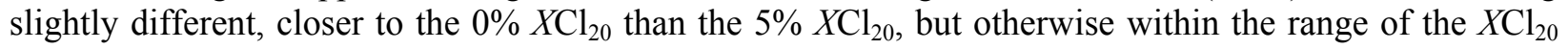
glasses. 
To further understand this behavior, ${ }^{207} \mathrm{~Pb}$ NMR spectra were collected on a subset of samples. The ${ }^{207} \mathrm{~Pb}$ NMR spectra consist of broad lineshapes $\sim 3000 \mathrm{ppm}$ wide which can be deconvoluted into two $\mathrm{Pb}$ sites, as shown for example in Figure 4.43. Both sites have negative chemical shifts, characteristic of ionically bonded $\mathrm{Pb}$ compounds (Fayon et al., 1997). Ionically bonded $\mathrm{PbCl}_{2}$ has a $\delta_{\text {iso }}$ of $-1717 \mathrm{ppm}$ and a $\mathrm{Pb}$ coordination number of seven, while covalently bonded $\mathrm{PbO}$ has a $\delta_{\text {iso }}$ of $1939 \mathrm{ppm}$ (red tetragonal litharge) or $1515 \mathrm{ppm}$ (yellow orthorhombic massicot) and coordination number of four (Fayon et al., 1997). Contrary to the results for the Te- $\mathrm{Pb}-\mathrm{O}-\mathrm{X}-\mathrm{Cl}$ glasses here, $\mathrm{Cd}-\mathrm{Pb}-\mathrm{O}-\mathrm{Cl}$ glasses show a mixed bonding character for $\mathrm{Pb}$ in $\mathrm{NMR}$, with a peak characteristic of covalent $\mathrm{Pb}-\mathrm{O}$ (positive chemical shift) and one of ionic $\mathrm{Pb}-\mathrm{Cl}$ (negative chemical shift) (Montagne et al., 2001). Empirical correlations between $\delta_{\text {iso, }}$, coordination number around lead, and $\mathrm{Pb}-\mathrm{O}$ bond distance suggest that the two sites in our Te-Pb-O$X-\mathrm{Cl}$ glass likely represent 5 - and 6-coordinated $\mathrm{Pb}$ with $\mathrm{Pb}-\mathrm{O}$ distance of $0.252-0.257 \mathrm{~nm}$ (Fayon et al., 1997). Note that two sites were present even in the glass without chlorides. Bond lengths and coordination for the $\mathrm{TeO}_{2}$ glasses suggest that some $\mathrm{PbO}_{a} \mathrm{Cl}_{b}$ octahedra exist, similar to crystal forms of $\mathrm{Pb}-\mathrm{O}-\mathrm{Cl}$ such as mendipite $\left(\mathrm{Pb}_{3} \mathrm{O}_{2} \mathrm{Cl}_{2}\right)$ (Lezal et al., 2002; Montagne et al., 2001; Porter and Halasyamani, 2003). In mendipite, $\mathrm{Pb}$ atoms are 6- or 7-coordinated with the first $\mathrm{Pb}$ coordination sphere containing oxygen at $\mathrm{Pb}-\mathrm{O} \sim 0.23 \mathrm{~nm}$ and chlorine at $\mathrm{Pb}-\mathrm{Cl} \sim 0.31 \mathrm{~nm}$ (Montagne et al., 2001).

The relative fraction of the two $\mathrm{Pb}$ sites changes monotonically with $X \mathrm{Cl}_{20}$ addition (Table 4.20) with a noticeable jog in the trend between $14 \%$ and $15 \% X_{\mathrm{Cl}_{20}}$, the same region of the phase separation. The $\sim 11 \%$ fraction of $\mathrm{Pb}$ site $1\left(\delta_{\text {iso }}=-1371 \mathrm{ppm}\right.$ at $\left.X \mathrm{Cl}_{20}=0\right)$ increases to $\sim 40 \%$ fraction at the highest chloride loading measured $\left(\delta_{\text {iso }}=-1168 \mathrm{ppm}\right.$ at $\left.X \mathrm{Cl}_{20}=17.5 \%\right)$, with a complementary decrease of the $\mathrm{Pb}$ site $2\left(\delta_{\text {iso }}=-1402 \mathrm{ppm}\right.$ at $\left.X \mathrm{Cl}_{20}=0\right)($ see Figure 4.45$)$. This result, combined with the lack of change in the ${ }^{125} \mathrm{Te}$ NMR, indicates that the addition of the alkali, alkaline earth, and lanthanide chlorides primarily effects the $\mathrm{Pb}$ environment and not the Te environment.

Table 4.20. ${ }^{207} \mathrm{~Pb}$ static NMR fit results.

\begin{tabular}{c|cccc|cccc}
\hline \multirow{2}{*}{$\begin{array}{c}\boldsymbol{X C l}_{\mathbf{2 0}} \\
(\mathbf{m a s} \%)\end{array}$} & \multicolumn{4}{|c|}{ Site 1 } & \multicolumn{4}{c}{ Site 2 } \\
\cline { 2 - 9 } & $\boldsymbol{\delta}_{\text {iso }}$ & $\boldsymbol{\Delta \boldsymbol { \delta }}$ & $\boldsymbol{\eta}$ & $\mathbf{A r e a \%}$ & $\boldsymbol{\delta}_{\text {iso }}$ & $\boldsymbol{\Delta \boldsymbol { \delta }}$ & $\boldsymbol{\eta}$ & Area\% \\
\hline 0 & -1371 & 1015 & 0.438 & 10.9 & -1402 & 2555 & 0.321 & 89.1 \\
10 & -1082 & 1059 & 0.604 & 19.4 & -1240 & 2526 & 0.361 & 80.6 \\
14 & -1059 & 1085 & 0.670 & 25.7 & -1272 & 2352 & 0.416 & 74.3 \\
15 & -1129 & 1101 & 0.677 & 35.1 & -1350 & 2384 & 0.382 & 64.9 \\
17.5 & -1168 & 1150 & 0.850 & 39.0 & -1316 & 2358 & 0.453 & 61.0 \\
\hline $14($ high $\mathrm{Pb})$ & -906 & 2649 & 0.925 & 26.2 & -1157 & 2088 & 0.530 & 73.8 \\
\hline
\end{tabular}

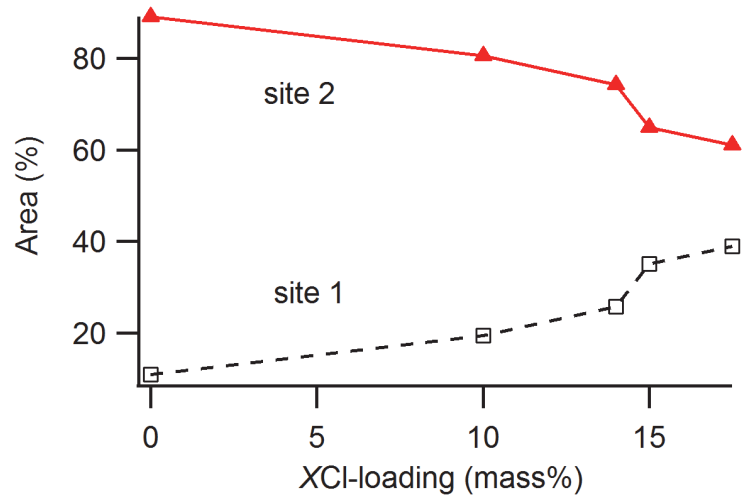

Figure 4.45. Variation of $\mathrm{Pb}$ site distribution with $X \mathrm{Cl}_{20}$-loading.

From the ${ }^{23} \mathrm{Na}$ data in Figure 4.44, it is apparent that there is one disordered Na site at the 5, 10, and 12.5 mass $\% X_{20}$ level, with a possible shoulder $\sim-16 \mathrm{ppm}$ in the latter glass. At $14 \mathrm{mass} \%$, the threshold 
phase separation, a peak is present at $7 \mathrm{ppm}$ that becomes very prominent in loadings $\geq 15$ mass $\%$. Additionally, a peak at $\sim-6 \mathrm{ppm}$ becomes apparent at $\geq 15$ mass $\%$, potentially shifting $\pm 1 \mathrm{ppm}$ up to the highest level of 22.5 mass\%. The peak at $7 \mathrm{ppm}$ is tentatively identified as $\mathrm{NaCl}$, and that at $-6 \mathrm{ppm}$ as $\mathrm{NaClO}_{4}$. The trend in line shape clearly shows the appearance of sharp, crystalline peaks related to $\mathrm{Na}$ phases beginning $\sim 14$ mass $\% X \mathrm{Cl}_{20}$ loading.

The ${ }^{35} \mathrm{Cl}$ data shown in Figure 4.44, suggests a similar behavior to the ${ }^{23} \mathrm{Na}$. A broad distribution of chemical shift is evident from 5-14 mass $\% X \mathrm{Cl}_{20}$, centered $\sim-8 \mathrm{ppm}$. In the $15-30$ mass $\% \mathrm{XCl}_{20}$ range, the distribution of chemical shift narrows considerably, again suggesting that chlorine is occupying a narrower range of sites. Taken together with the ${ }^{23} \mathrm{Na}$ data, the ${ }^{35} \mathrm{Cl}$ data indicates that crystalline, or at least highly ordered, chlorine sites are increasingly present as $X \mathrm{Cl}_{20}$ is increased above 14 mass\%.

\subsection{Tellurite Glasses - Overview}

No studies of tellurite glasses exist in the literature with the complexity of constituents as considered here, including both a modifier (and co-glass former) like $\mathrm{PbO}$ and multiple alkali, alkaline earth, and lanthanide chlorides. Therefore, comparison must be made to simpler systems to gain corroboratory insight into the glass structure change with composition.

Several studies have investigated binary compositions of metal chlorides with $\mathrm{TeO}_{2}$. Notably, neutron diffraction experiments of $\mathrm{TeO}_{2}$ with up to $35 \mathrm{~mol} \% \mathrm{BiCl}_{3}$ (Neov et al., 1990) or $20 \mathrm{~mol} \% \mathrm{CsCl}, \mathrm{MnCl}_{2}$, $\mathrm{FeCl}_{2}$, or $\mathrm{FeCl}_{3}$ (Neov et al., 1995) noted no change in the tellurite structural configuration from 4coordination, suggesting that the chlorides exist quasi-independently of the tellurite network and do not participate in $t b p$ network breaking. In other words, the salt exists as an independent phase-separated entity on the local level. Very few studies exist in the literature on tellurite glasses with oxide modifiers in addition to halide modifiers. Recently, Solokov et al. studied $\mathrm{TeO}_{2}-\mathrm{BaO}-\mathrm{BaCl}_{2}$ systems, both experimentally and computationally, and concluded that the barium and chlorine were present as free ions in the interstices of the tellurite matrix, as $\mathrm{Ba}^{2+}$ and $\mathrm{Cl}^{-}$(Sokolov et al., 2009). In comparing the effects of $\mathrm{BaO}$ with $\mathrm{BaCl}_{2}$ using ab inito molecular dynamics simulations, the authors state that each $\mathrm{Ba}$ atom, when added as an oxide, forms two $N_{4}^{-}$species and one $N_{3}^{-}$species, whereas when it is added as a chloride, it forms three $N_{3}^{-}$species. In both cases, this indicates a depolymerization of the $N_{4}^{0}$ tellurite network.

These two examples illustrate the two models usually offered for structure of halide-containing oxide glass systems (Chowdari et al., 1998). In the first case with $\mathrm{TeO}_{2}+$ chlorides (Neov et al., 1995; Neov et al., 1990), the salt phase can be understood as being composed of clusters in the interstices of the host oxide glass which do not modify the glass structure. Alternatively, the glass structure may change systematically with the halide salt dopant, as is the case in $\mathrm{TeO}_{2}-\mathrm{BaO}-\mathrm{BaCl}_{2}$ (Sokolov et al., 2009) and $\mathrm{TeO}_{2}-\mathrm{PbF}_{2}$ (Kabalc1 et al., 2009). Similarly, a $\mathrm{TeO}_{2}-\mathrm{CuI}-\mathrm{Cu}_{2} \mathrm{O}$ glass was observed to phase separate, causing difficulty in interpretation of glass transition and crystallization processes (Chowdari et al., 2000). These authors reported 60-920 nm phase-separation in the form of droplets and clusters where the size was composition-dependent.

In the case of the $\mathrm{TeO}_{2}-\mathrm{PbO}-X \mathrm{Cl}$ system studied here, interpreting the Raman data alone suggests that the increase of the $X \mathrm{Cl}_{20}$ mixed salt dopant resulted in a progressive depolymerization of the glass network up to the point of $\sim 14$ mass $\% X \mathrm{Cl}_{20}$. However, ${ }^{125} \mathrm{Te}$ NMR showed no change in the site distribution of Te, rather suggesting that the polymerization of the glass network was fixed by the Te:Pb ratio, which remained constant. An alternative interpretation of the Raman data is that the local vibrational modes for $t p$ and $t b p$ change as a function of composition due to the near-presence of increasing amounts of alkali metals and chlorine. The ${ }^{23} \mathrm{Na}$ and ${ }^{35} \mathrm{Cl} \mathrm{NMR}$ show a broad distribution of $\mathrm{Na}$ sites up to 14 mass\%, indicating that $\mathrm{Na}$ and $\mathrm{Cl}$ are present in the glass structure in a variety of sites. The Raman vibrations of the tellurite units could change as a function of composition due to these additional ions, even if the site distribution of Te sites is not effected. A small peak in the ${ }^{23} \mathrm{Na}$ NMR at 14 mass $\%$ seems to correlate with the low concentration of crystalline or weakly crystalline inclusions seen in TEM in this specimen. 
Above 14 mass $\% \mathrm{XCl}_{20}$, SEM, TEM, XRD, and ${ }^{23} \mathrm{Na}$ and ${ }^{35} \mathrm{Cl} \mathrm{NMR}$ all support the progressive formation of crystalline salt phases. Sharp peaks in ${ }^{23} \mathrm{Na}$ suggest multiple crystalline Na phases are present, and the ${ }^{35} \mathrm{Cl}$ spectrum progressively narrows. It is unclear whether the mechanism is phase separation in the melt followed by crystallization, or crystallization out of the melt. At the highest salt doping level of 22.5 mass $\%$, the mole fraction of the $\mathrm{TeO}_{2}$ is roughly equal to that of $X \mathrm{Cl}_{20}$, and a partially percolated network of the salt is realized. Up to the solubility of the mixed salt phase in the glass $\left(\sim 15\right.$ mass $\left.\% X \mathrm{Cl}_{20}\right)$, the salt systematically changes the tellurite glass structure by incorporating $\mathrm{Cl}, \mathrm{Na}$, and probably other cations, but does not affect the Te site distribution. Above this solubility, the excess salt no longer systematically affects the local environment (and hence vibrational states) of the glass network but phase separates and crystallizes, and the glass vibrational structure remains essentially as it was at the 15 mass $\% \mathrm{XCl}_{20}$ level. The remaining salt phase can be thought of as being distributed in the interstices of the glass network.

The role of the $\mathrm{Pb}$ in this glass system should not be discounted, especially since it appears that the $\mathrm{Pb}$ determines the Te site distribution. Additionally, the ${ }^{207} \mathrm{~Pb}$ NMR shows that the distribution of the two $\mathrm{Pb}$ sites changes significantly with the addition of mixed salt, favoring site 1 (more negative chemical shift, hence more ionic) at higher loadings. Lead also helps to polymerize the network (probably as $\mathrm{PbO}_{a} \mathrm{Cl}_{b}$ octahedra), keeping the glass network continuous when it might otherwise be completely broken. One can envision $\mathrm{Te}-\mathrm{Cl}$ bonds (Lezal et al., 2002), particularly $\mathrm{Te}-\mathrm{Cl}_{\mathrm{eq}}$ that may strengthen $\mathrm{Te}-\mathrm{O}_{\mathrm{ax}}$ and prevent some $t p$ formation (Tanaka et al., 1988b), $\mathrm{Pb}-\mathrm{Cl}$ bonds (at least in the compositions with less $X \mathrm{Cl}_{20}$ ), and $\mathrm{Te}-\mathrm{O}-\mathrm{Pb}$ bonds (Silva et al., 2001). Given their size (see Table 2.2), the lanthanides are likely in interstices in the tellurite or lead networks (Nachimuthu and Jagannathan, 1995), but recent results on $\mathrm{TeO}_{2}-\mathrm{PbO}-\mathrm{Eu}_{2} \mathrm{O}_{3}$ oxide glasses indicate that $\mathrm{Eu}$, at significant concentrations, can favor the creation of $\mathrm{PbO}_{4}$ units in tellurite glass (Rada et al., 2011).

It is likely that the combination of highly polarizable $\mathrm{Te}$ and $\mathrm{Pb}$ allow for large salt concentrations. Both $\mathrm{Te}^{4+}$ and $\mathrm{Pb}^{2+}$ have a lone pair of electrons that result in a highly polar asymmetry and, in some Te-Pb-O$\mathrm{Cl}$ crystals, the lone pairs point toward the chlorine atoms in the structure to minimize electrostatic effects (Porter and Halasyamani, 2003). A comparison of glasses with the same cation molar concentrations but without $\mathrm{Cl}$ (e.g., T2G-34 vs. T2G-22), suggests that $t b p / t p$ ratios increase when $\mathrm{Cl}$ is substituted for $\mathrm{O}$, indicating that $\mathrm{Cl}$-containing glasses are relatively more polymerized than their oxygen counterparts. This could be due to the scavenging effects of $\mathrm{Cl}$ on alkalis, preventing the incorporation of some $\mathrm{Na}$ and $\mathrm{Li}$ into the glass. This appears to be due to an ionic component of charge compensation.

Previous results with the 10 mass $\% \mathrm{XCl}_{20}$ loading showed very high chemical durability for the $\mathrm{TeO}_{2}$ $\mathrm{PbO}-\mathrm{XCl}$ glasses compared to other candidates (Riley et al., 2010; Riley et al., 2012b). This effort was focused on determining the maximum $X \mathrm{Cl}_{20}$-loading for this particular glass system. It appears that the $\mathrm{TeO}_{2}-\mathrm{PbO}-\mathrm{XCl}$ system with 14 mass $\% \mathrm{XCl}_{20}$ is a highly metastable system, with energy from an electron microscope being sufficient to induce phase separation and crystallization. This suggests that 14 mass $\%$ $\mathrm{XCl}_{20}$-loading is the upper limit for use as a waste form with this particular laboratory quenching method and batch size. It may be that waste forms processed at a larger scale, resulting in slower cooling in some portions, would result in faster crystallization and thus lower loading limit (based on phase separation). Additionally, it is noteworthy that the actual chloride stream will contain some radioactivity that will add energy to the system, thus potentially promoting phase separation and crystallization. It is presumed that the chemical durability of the phase separated and crystallized glass would be lower than uncrystallized glass because of the water-soluble, salt-rich phases observed here as found in other systems (Rodriguez et al., 2011), but this has not been verified for the $\mathrm{TeO}_{2}-\mathrm{PbO}-X \mathrm{Cl}$ system.

\subsection{Polycrystalline Tellurite Compounds as Standards}

In FY2011, five tellurite crystals were fabricated and included: $\mathrm{ZnTeO}_{3}, \mathrm{Zn}_{2} \mathrm{Te}_{3} \mathrm{O}_{8}, \mathrm{SnTe}_{3} \mathrm{O}_{8}, \mathrm{ZrTe}_{3} \mathrm{O}_{8}$, and $\mathrm{TiTe}_{3} \mathrm{O}_{8}$. The procedures used to fabricate these samples were first attempted with those laid out in Table 3.12, but modifications were made in some cases. Pictures of these crystals are presented in Figure 4.46. It was difficult to achieve $100 \%$ of the target phases in all cases without at least minor impurities 
from unincorporated reactants with the exception of $\mathrm{ZnTeO}_{3}$. A summary of the crystalline phases found in these samples during XRD analysis is presented in Table 4.21.
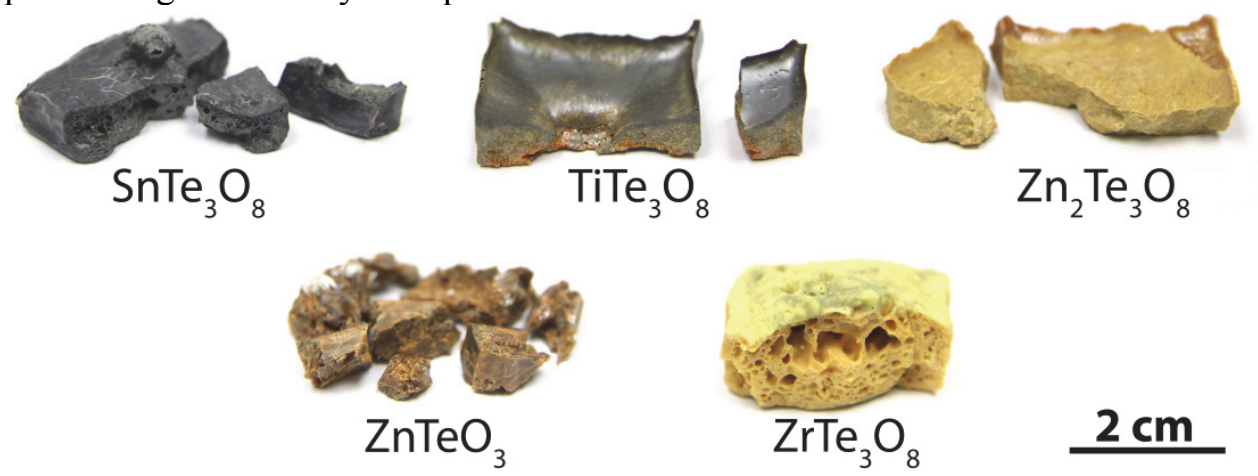

Figure 4.46. Pictures of the tellurite crystals fabricated in FY2011. The scale-bar is the same for all micrographs.

Table 4.21. Summary of crystalline phases identified in tellurite crystals.

\begin{tabular}{lllll}
\hline $\mathrm{Phase}$ & $\mathrm{PDF} \#$ & ICSD\# & $\mathrm{SG}(\mathrm{SG} \#)$ & Reference \\
\hline $\mathrm{SnTe}_{3} \mathrm{O}_{8}$ & $70-2440$ & 9077 & $I a-3(206)$ & (Meunier and Galy, 1971) \\
$\mathrm{TiTe}_{3} \mathrm{O}_{8}$ & $70-2439$ & 9076 & $I a-3(206)$ & (Meunier and Galy, 1971) \\
$\mathrm{ZnTeO}_{3}$ & $44-0240$ & N/A & $P b c a(61)$ & (a) \\
$\mathrm{ZnTeO}_{3}$ & $72-1410$ & 16937 & $P b c a(61)$ & (Hanke, 1967) \\
$\mathrm{Zn}_{2} \mathrm{Te}_{3} \mathrm{O}_{8}$ & $89-4454$ & 50705 & $\mathrm{C} 2 / c(15)$ & (Feger et al., 1999) \\
$\mathrm{ZrTe}_{3} \mathrm{O}_{8}$ & $70-2442$ & 9079 & $I a-3(206)$ & (Meunier and Galy, 1971) \\
$\mathrm{SnO}_{2}$ & $70-6153$ & 91517 & $P 42 / m n m(136)$ & (Kim et al., 2000) \\
$\mathrm{SnO}_{2}$ & $41-1445$ & N/A & $P 42 / m n m(136)$ & (McCarthy and Welton, 1989) \\
$\alpha-\mathrm{TeO}_{2}$ & $42-1365$ & 27515 & $P 41212(92)$ & (b) \\
$\alpha-\mathrm{TeO}_{2}$ & $74-0269$ & 25706 & $P 41212(92)$ & (Leciejewicz, 1961) \\
$\mathrm{TiO}_{2}$ & $70-7347$ & 93097 & $P 42 / m n m(136)$ & (Ballirano and Caminiti, 2001) \\
$\mathrm{ZrO}_{2}$ & $89-9069$ & 89429 & $F m-3 m(225)$ & (Wang et al., 1999)
\end{tabular}

${ }^{(a)}$ Rosenstingl, J., CH Hoffmann, E Irran, CH Lengauer, E Tillmanns, Vienna, Austria. ICDD Grant-in-Aid (1992)

(b) Information given in PDF card: Blanchard, F., Department of Geology, University of Florida, Gainesville (1990).

\subsection{1 $\mathrm{TeO}_{3}$-Isolated Crystals}

The only crystal in this category that was made successfully was the $\mathrm{ZnTeO}_{3}$ crystal, which we made as phase-pure because all of the diffraction peaks fit well to the ICSD standard in the database. The XRD pattern is presented in Figure 4.47. This sample seemed to be the most brittle of the five that we made. 


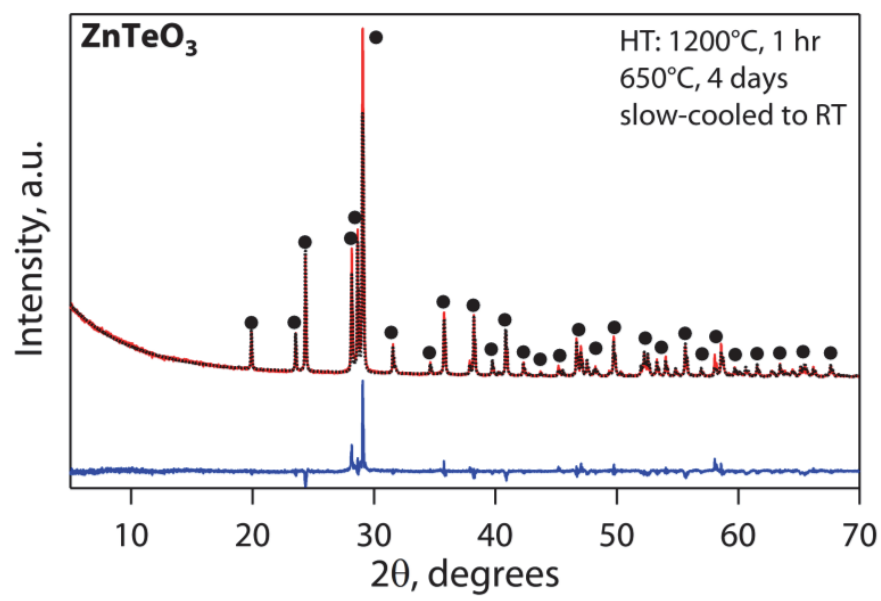

Figure 4.47. Rietveld refinement on $\mathrm{ZnTeO}_{3}$ crystals showing the measured, calculated, and difference spectra. Here, represents the target phase $\left(\mathrm{ZnTeO}_{3}, \mathrm{PDF} \# 72-1410\right.$, Table 4.21$)$.

\subsection{2 $\mathrm{TeO}_{3}$-Terminal Crystals}

The only crystal that was attempted in the class of $\mathrm{TeO}_{3}$-terminal crystals was $\mathrm{Zn}_{2} \mathrm{Te}_{3} \mathrm{O}_{8}$; this was achieved at high purity levels with minor impurities of what appeared to be the $\mathrm{ZnTeO}_{3}$ phase. The Rietveld refinement of this pattern is presented in Figure 4.49.

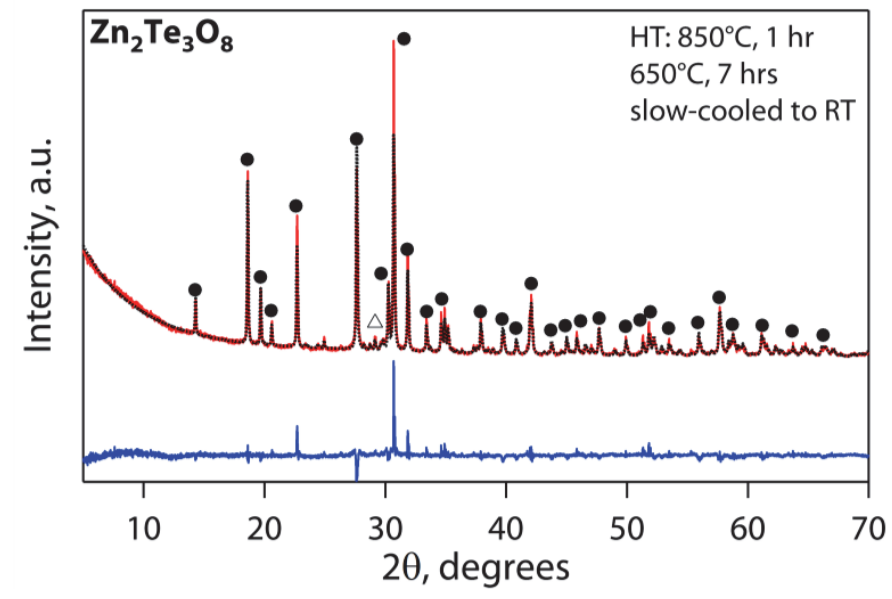

Figure 4.48. Rietveld refinement on $\mathrm{Zn}_{2} \mathrm{Te}_{3} \mathrm{O}_{8}$ crystals showing the measured, calculated, and difference spectra. Here, $\bigcirc$ represents the target phase $\left(\mathrm{Zn}_{2} \mathrm{Te}_{3} \mathrm{O}_{8}, \mathrm{PDF} \# 89-4454\right)$ at 97.08 mass $\%$ and $\triangle$ represents a minor impurity phase, $\mathrm{ZnTeO}_{3}(\mathrm{PDF} \# 75-1410)$ at 2.92 mass\% (Table 4.21).

\subsection{3 $\alpha-\mathrm{TeO}_{2}$ Tellurite Crystals}

Three phases in this class of materials were investigated in FY2011: $\mathrm{ZrTe}_{3} \mathrm{O}_{8}, \mathrm{SnTe}_{3} \mathrm{O}_{8}$, and $\mathrm{TiTe}_{3} \mathrm{O}_{8}$. $\mathrm{SnTe}_{3} \mathrm{O}_{8}$ and $\mathrm{TiTe}_{3} \mathrm{O}_{8}$ were both heat-treated at $650{ }^{\circ} \mathrm{C}$ for several days and did not melt, although they did result in relatively pure materials of the target compounds. However, the target goal was to melt the mixtures and fire them to both achieve phase-pure materials of the target composition and have particles large enough to size for chemical durability tests if they are sieved to isolate the larger particle sizes. Thus, high-temperature $\left(T>900{ }^{\circ} \mathrm{C}\right)$ soaks were attempted prior to the lower temperature heat treatment for a long duration. These heat treatments did not always have the desired results; in some cases heat treatment at these higher temperatures resulted in a decrease of the target phase. For the $\mathrm{SnTe}_{3} \mathrm{O}_{8}$ and $\mathrm{TiTe}_{3} \mathrm{O}_{8}$ samples, the Rietveld refinements of the XRD data are presented in Figure 4.49. 

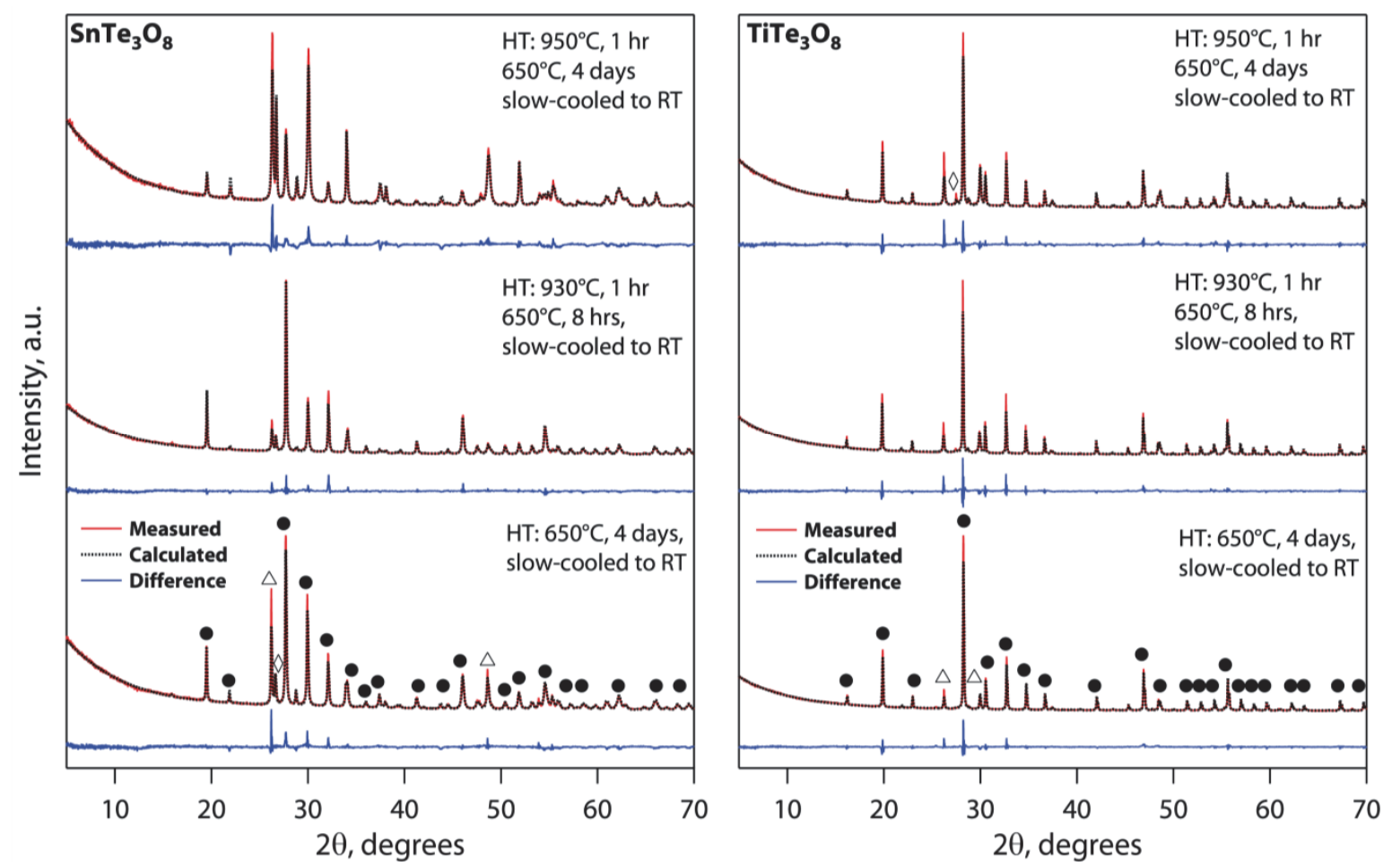

Figure 4.49. Rietveld refinement of $\mathrm{XRD}$ spectra for the $\mathrm{SnTe}_{3} \mathrm{O}_{8}$ (left) and $\mathrm{TiTe}_{3} \mathrm{O}_{8}$ (right) samples after various heat treatments. The samples were run sequentially from the bottom spectra toward the top spectra. Here, represents the target phases of $\mathrm{SnTe}_{3} \mathrm{O}_{8}$ (PDF\#70-2440) and $\mathrm{TiTe}_{3} \mathrm{O}_{8}$ (PDF\#70-2439), $\triangle$ represents $\alpha-\mathrm{TeO}_{2}$ (PDF\#42-1365), and $\diamond$ represents the metal oxides of $\mathrm{SnO}_{2}(\mathrm{PDF} \# 70-6153)$ and $\mathrm{TiO}_{2}$ (PDF\#70-7347) in the corresponding figures (right and left, respectively) (see Table 4.21). The quantities of the constituents of these samples after each heat treatment are presented in Table 4.22.

Table 4.22. Mass\% of components in $\mathrm{SnTe}_{3} \mathrm{O}_{8}$ and $\mathrm{TiTe}_{3} \mathrm{O}_{8}$ samples after Rietveld refinements. The phases correspond to phases in Table 4.21 with graphical representations in Figure 4.49. The temperatures correspond to the heat treatment temperatures.

\begin{tabular}{l|ccc|ccc}
\hline & \multicolumn{3}{|c|}{$\mathrm{SnTe}_{3} \mathrm{O}_{8}$} & \multicolumn{3}{c}{$\mathrm{TiTe}_{3} \mathrm{O}_{8}$} \\
\hline Components & $650{ }^{\circ} \mathrm{C}$ & $930{ }^{\circ} \mathrm{C}$ & $950{ }^{\circ} \mathrm{C}$ & $650{ }^{\circ} \mathrm{C}$ & $930{ }^{\circ} \mathrm{C}$ & $950{ }^{\circ} \mathrm{C}$ \\
\hline $\mathrm{SnTe}_{3} \mathrm{O}_{8}$ & 56.83 & 75.32 & 23.84 & - & - & - \\
$\mathrm{SnO}_{2}$ & 11.41 & 6.59 & 20.12 & - & - & - \\
$\mathrm{TeO}_{2}$ & 31.76 & 18.09 & 56.04 & 10.07 & 17.33 & 23.1 \\
$\mathrm{TiTe}_{3} \mathrm{O}_{8}$ & - & - & - & 84.95 & 73.46 & 69.38 \\
$\mathrm{TiO}_{2}$ & - & - & - & 4.98 & 9.21 & 7.52 \\
\hline
\end{tabular}

For the $\mathrm{ZrTe}_{3} \mathrm{O}_{8}$ sample, the single heat treatment at $950{ }^{\circ} \mathrm{C}$ resulted in a very highly pure sample with only minor impurities of the unincorporated reactants. The Rietveld refinement of that sample is presented in Figure 4.50. 


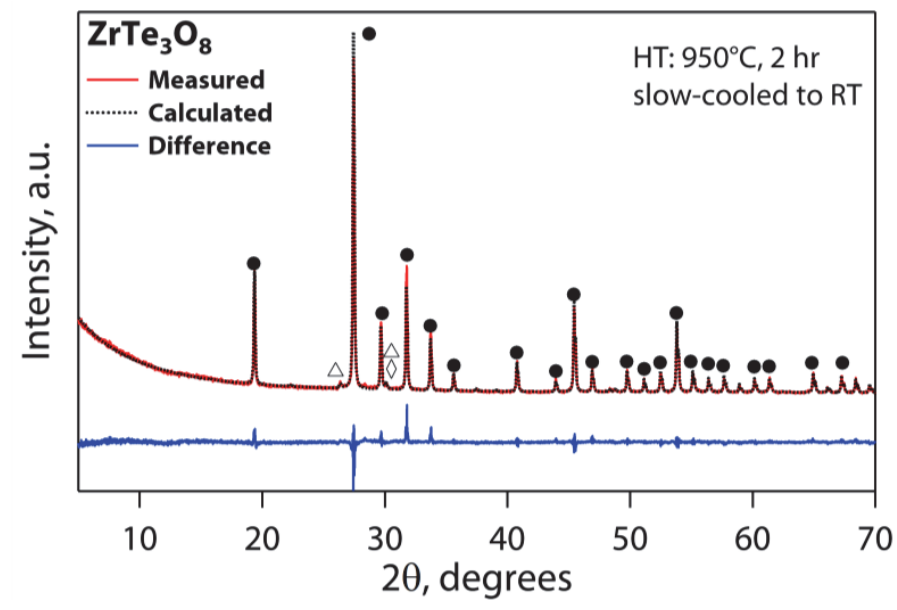

Figure 4.50. Rietveld refinement of XRD spectra for $\mathrm{ZrTe}_{3} \mathrm{O}_{8}$ after the $950{ }^{\circ} \mathrm{C}$ heat treatment. Here, represents the target phase $\left(\mathrm{ZrTe}_{3} \mathrm{O}_{8}, \mathrm{PDF} \# 70-2442\right), \triangle$ represents $\alpha-\mathrm{TeO}_{2}(\mathrm{PDF} 442-1365)$, and $\diamond$ represents $\mathrm{ZrO}_{2}$ (PDF\#89-9069). Here, the $\mathrm{ZrTe}_{3} \mathrm{O}_{8}$ phase represents 97.21 mass $\%$ and $\mathrm{TeO}_{2}$ represents 2.79 mass $\%$ of the crystalline content.

According to the results this far, it is apparent that it is possible to fabricate melted solids of highly pure tellurite crystals that can be used for chemical durability testing. This was a scoping study to see if it was possible to make solids of these crystals so they could be powdered after achieving phase-pure samples and then sized for chemical durability testing. 


\section{CONCLUSIONS}

\subsection{Tellurite Glasses}

We performed several concurrent studies with the tellurite glass waste form in FY2011 and FY2012. These studies included the following: 1) structural investigations on the P-series tellurite glasses from FY2010, 2) an expanded study on the lead-tellurite glasses that had high chemical durability in the FY2010 study, 3) a study on a series of glasses designed to reveal the glass-structural effects of anion/cation substitutions into the tellurite glass network, and 4) a study on the chemical durability of tellurite crystals with known crystal structure and tellurium-oxygen coordination.

Study 1 - Lead-tellurite variation glasses. A series of $\mathrm{TeO}_{2}-\mathrm{PbO}$ glasses were fabricated with various amounts of a mixed chloride waste simulant (nonradioactive) intended to represent the EChem waste stream from the electrochemical process used for processing nuclear fuel. The structure of these glasses was studied by characterization with nuclear magnetic resonance, Raman spectroscopy and X-ray diffraction, and indicated that the medium-range order in the glass decreased and the tellurite network was depolymerized as the waste fraction was increased. It was apparent that glass network-breaking was predominantly caused by the additions of the waste cations and that the presence of chloride may have actually caused some repolymerization of the network by scavenging alkalis and preventing it from acting as a network modifier. Under the processing conditions used in this study, the waste loading limit was determined to be around 14 mass $\% X \mathrm{Cl}_{20}$, above which phase separation was observed resulting in a Terich phase and a alkali-chloride-rich phase, and some chloride-containing crystals.

Study 2 - Compositional variation glasses. In this study, which was the ground work for Study 2, we learned the various interpretations of the diverse set of Raman spectra observed in tellurite glasses. We measured the Raman spectra for all 10 of the P-series glasses that were analyzed with the PCT. The primary goal of the structural interrogation of the P-series glasses was to see whether we could find a correlation between the tellurium-oxygen coordination environment and the chemical durability, considering that the chemical durability of these glasses was so diverse. However, we failed to find a simple correlation.

Study 3 - Structural variation glasses. A series of glasses were designed to investigate the glass structural effects when select species in the glasses from Study 1 and Study 2 were substituted for other species. The aim of these studies was to better understand the difference between the roles of the cations and anions from both the lead and the $X \mathrm{Cl}_{20}$ components. We looked at (1) lead-tellurite glasses with $\mathrm{K}_{2} \mathrm{O}$ or KCl, (2) a lead-tellurite glass in which $X \mathrm{Cl}_{20}$ components were substituted for $X \mathrm{O}_{20}$ components ("X" cation ratios to $\mathrm{Te}$ and $\mathrm{Pb}$ retained from the $\mathrm{TPb}_{5}$ glass), and (3) some lead-tellurite glasses in which $\mathrm{PbCl}_{2}$ was substituted for PbO. Structural analysis on these glasses indicated that the chloride in $X \mathrm{Cl}_{20}$ actually repolymerizes the tellurite network compared to the oxygen in the $X \mathrm{O}_{20}$ glass with the same modifier cations, by scavenging some of the alkalis into a separate phase that is ultimately present as nano-phase-separated droplets at the highest $\mathrm{XCl}_{20}$-loadings. This local removal of alkali from the role of network modifier of the tellurite network, even in the low waste loading glasses devoid of visible phase separation, is believed to be the mechanism responsible for preventing formation of some tp units indicative of depolymerization. The comparison of the $\mathrm{K}_{2} \mathrm{O}$ and $\mathrm{KCl}$ glasses having the same relative amount of $\mathrm{K}$ compared to $\mathrm{Te}$ and $\mathrm{Pb}$ indicated the same trend, i.e., that the chloride-containing glass is slightly more polymerized than the one without chloride when both contain the same relative amount of alkali to the main glass formers.

Study 4 - Polycrystalline tellurite compounds as standards. A study on tellurite crystals was identified in the literature as a potentially valuable contribution to our work. In this study, a wide range of binary and ternary Te-O crystals were made and categorized into groups in which the structures were composed of either $\mathrm{TeO}_{3}, \mathrm{TeO}_{3+1}, \alpha-\mathrm{TeO}_{2}$, or $\beta-\mathrm{TeO}_{2} \mathrm{Te}-\mathrm{O}$ structural units. The primary goal of pursuing these was to make a few of the crystals in each "category" and test the chemical durability of the crystals. If one of 
these structural units is preferred for high chemical durability, then since the crystals are composed of a single structural unit throughout, crystals from certain categories may show better chemical durability than others. We successfully made five crystals, some of them nearly phase-pure.

\subsection{Halide Minerals}

In order to improve upon our results from past years on solution-derived sodalites, we made some changes to the process in FY2011. These changes included the following: (1) replacing CS with TEOS as the silica source in the process, (2) replacing Si with Ge in the sodalite with germanium (IV) ethoxide, and (3) optimizing the sintering aid for low-temperature firing to reduce chloride volatility and maximize sodalite production.

Study 1 - Organic silica source. We replaced the CS with TEOS as the silica source in the process to see whether it altered the reaction rate, allowing for a higher concentration of sodalite in the dried product $\left(90{ }^{\circ} \mathrm{C}\right)$. The change was a noticeable improvement for the dried product from 76 mass\% sodalite in the S5A specimens with CS to 96 mass\% sodalite in the S5C specimen with TEOS.

Study 2 - Organic germania source. Here, we wanted to look at an organic source of germania as a replacement for silica, i.e., germanium (IV) ethoxide. The primary goal of this substitution was that we predicted that the germania-based gel, when pressed into pellets, would sinter at a lower temperature and that would reduce the $\mathrm{Cl}$ volatility and increase the density of the fired pellets for a higher volumetric waste loading. The drawback with exchanging $\mathrm{Si}$ for $\mathrm{Ge}$ is the increased costs associated with Ge. However, we wanted to explore this potential option. The high porosity in the initially fired pellets was a deterrent to pursuing this route any farther, though we did demonstrate a high germania-based sodalite concentration ( 82 mass $\%$ ) in the as-made gel.

Study 3 - Alternative sintering aids. Here, we investigated alternative glass-binders to the NBS-1 glass. Since the viscosity of the NBS-1 glass is so high, the temperatures required for pellet densification were rather high $\left(950^{\circ} \mathrm{C}\right)$ and that led to chloride volatility, evidenced by a decrease in the sodalite concentration. In order to reduce the sintering temperatures, six alternative sintering aids were fabricated and added to the solution during the process. These sintering aids included four sodium borosilicate formulations, a sodium aluminophosphate formulation, and a lead-tellurite glass formulation $\left(\mathrm{TPb}_{0}\right)$. These samples were dried, pressed into pellets, fired at different temperatures, and the phases quantified. The sodium borosilicate glasses proved to dramatically improve the densification, up to $92 \%$ of $\rho_{\mathrm{t}}$, of the fired pellets at much-reduced temperatures of $\sim 750{ }^{\circ} \mathrm{C}$, where reasonable amounts of sodalite were still produced ( 71 mass $\%)$.

In addition to adding a sintering aid to help consolidate the materials, we will be investigating alternative sintering techniques that include pressure control in order to maintain the stability of the sodalite structure. This will provide the ability to reduce porosity and increase density. We propose using sparkplasma sintering because it has high pressure capability plus the added benefit of voltage, which promotes grain bonding, resulting in reduced surface area. The spark-plasma sintering process is also rapid, $<1 \mathrm{~h}$, compared to traditional sintering which takes 10 to $24 \mathrm{~h}$ and this reduced heat-treatment time is expected to reduce chloride volatility.

Study 4 - Gibbsite (SM-1), NaAlO a different approach to making high density sodalite pellets. Three different solution-based formulations were evaluated to make sodalite with the same simulated $X \mathrm{Cl}_{20}$ salt. Several aspects were varied during the process including: the reactants, the amount of sintering aid added $(0,5$, and 10 mass $\%)$, the step in the process when the sintering aid was added, calcination, and the timing of the calcination step in the process. The three sodalite methods $(S M-\#)$ used in the current study are $S M-1, S M-2$, and $S M-3$, simplified as:

SM-1: $\quad 6 \mathrm{NaOH}+6 \mathrm{Al}(\mathrm{OH})_{3}+2 \mathrm{NaCl}+6 \mathrm{SiO}_{2} \rightarrow \mathrm{Na}_{8}\left(\mathrm{AlSiO}_{4}\right)_{6} \mathrm{Cl}_{2}+24 \mathrm{OH}^{-}$ 
SM-2:

$$
6 \mathrm{NaAlO}_{2}+2 \mathrm{NaCl}+6 \mathrm{SiO}_{2} \rightarrow \mathrm{Na}_{8}\left(\mathrm{AlSiO}_{4}\right)_{6} \mathrm{Cl}_{2}
$$

SM-3:

$$
6 \mathrm{NaOH}+3 \mathrm{Al}_{2} \mathrm{Si}_{2} \mathrm{O}_{7}+2 \mathrm{NaCl} \rightarrow \mathrm{Na}_{8}\left(\mathrm{AlSiO}_{4}\right)_{6} \mathrm{Cl}_{2}+6 \mathrm{OH}^{-}
$$

The equations above are listed with the salt being denoted as " $\mathrm{NaCl}$ " but, in fact, the actual salt compositions contain $\mathrm{R}^{1+}, \mathrm{R}^{2+}$, and $\mathrm{R}^{3+}$ cations so the $\mathrm{R}: \mathrm{Cl}$ ratio is not $1: 1$. The reactants were added in the proper amounts to obtain the targeted value for $\mathrm{Al}: \mathrm{Cl}(3: 1)$ and $\mathrm{Si}: \mathrm{Cl}(3: 1)$ where the alkali fraction $\left(\mathrm{R}^{1+}\right)$ was intentionally below the target of $4: 1\left(\mathrm{R}^{1+}: \mathrm{Cl}\right)$ to help influence the incorporation of alkaline earth and Ln components from the $X \mathrm{Cl}_{20}$ into the sodalite structure. The results from this study showed that each formulation had advantages and disadvantages where SM-3 pellets had high density and low sodalite production and SM-2 pellets had very high sodalite production with modestly high densities. A mixture of these methods will likely be considered in FY2013. 


\section{ACKNOWLEDGEMENTS}

Authors would like to thank the U.S. Department of Energy, Office of Nuclear Energy for their support of this work under Contract Number DE-AC05-76RL01830. Authors thank Ashutosh Goel, Nicole Overman, Joseph Westsik, and Loni Peurrung for helpful review of this document, James Bresee (DOE Office of Nuclear Energy) and Terry Todd (Idaho National Laboratory) for project oversight. The authors greatly thank Laura Buchanan for her hard work in making sure that the schedule and performance of milestones and monthly reports were carefully tracked, Teresa Schott for administrative support, and Maura Zimmerschied for her help with the manuscript. A portion of the research was performed using the Environmental Molecular Sciences Laboratory (EMSL), a national scientific user facility sponsored by the DOE's Office of Biological and Environmental Research and located at PNNL. 


\section{REFERENCES}

ASTM C 1285-08. 2008. Standard Test Methods for Determining Chemical Durability of Nuclear, Hazardous, and Mixed Waste Glasses and Multiphase Glass Ceramics: The Product Consistency Test (PCT), American Society for Testing and Materials International, West Conshohocken, PA.

Babad, H and DM Strachan. 1980. "Method for immobilizing radioactive iodine." US Patent No. 4229317.

Bak, M, JT Rasmussen, and NC Nielsen. 2000. "SIMPSON. A general simulation program for solidstate NMR spectroscopy." Journal of Magnetic Resonance 147(2):296-330.

Ballirano, P and R Caminiti. 2001. "Rietveld refinements on laboratory energy dispersive X-ray diffraction (EDXD) data." Journal of Applied Crystallography 34(6):757-62.

Barrett, WT and WE Wallace. 1954. "Studies of NaCl-KCl solid solutions. I. Heats of formation, lattice spacings, densities, Schottky defects and mutual solubilities." Journal of the American Chemical Society 76(2):366-69.

Barth, TFW. 1932a. "The structure of the minerals of the sodalite family." Zeitschrift für Kristallographie, Kristallgeometrie, Kristallphysik, Kristallchemie 83:405-14.

Barth, TFW. 1932b. "The structures of the minerals of the sodalite family." Z. Kristallogr., Kristallgeom., Kristallphys., Kristallchem. 83:405-14.

Bateman, KJ, CJ Knight, and CW Solbrig. 2007. Current Status of Ceramic Waste Form Development. Report No. INL/INT-06-11736, Rev. 1, Idaho National Laboratory, Idaho Falls, ID.

Beagley, B, CMB Henderson, and D Taylor. 1982. "The crystal structures of aluminoslicate-sodalites: X-ray diffraction studies and computer modelling." Mineralogical Magazine 46:459-64.

Beyer, H. 1967. "Verfeinerung der kristallstruktur von tellurit, dem rhombischen $\mathrm{TeO}_{2} . "$ Kristallografiya 124:228-38.

Brow, RK, DR Tallant, and GL Turner. 1997. "Polyhedral arrangements in lanthanum aluminoborate glasses." Journal of the American Ceramic Society 80(5):1239-44.

Brunauer, S, PH Emmett, and E Teller. 1938. "Adsorption of gases in multimolecular layers." Journal of the American Chemical Society 60(2):309-19.

Chowdari, BVR and PP Kumari. 1999. "Raman spectroscopic study of ternary silver tellurite glasses." Materials Research Bulletin 34(2):327-42.

Chowdari, BVR, KL Tan, and F Ling. 2000. "Synthesis and characterization of $\mathrm{Cu}_{2} \mathrm{O} \cdot \mathrm{TeO}_{2}$ and $\mathrm{CuI} \cdot \mathrm{Cu}_{2} \mathrm{O} \cdot \mathrm{TeO}_{2}$ glasses." J. Mater. Sci. 35:2015-27.

Chowdari, BVR, KL Tan, and F Ling. 1998. "Characterization of $x \mathrm{CuI}-(50-x) \mathrm{AgI}-y \mathrm{Cu}_{2} \mathrm{MoO}_{4}{ }^{-}$ $(50-y) \mathrm{CuPO}_{3}$ glasses." Solid State Ionics 107(1-2):89-103. 
Cressey, G. 2004. "Rock-forming minerals. Volume 4B. Second edition. framework silicates: Silica minerals, feldspathoids and the zeolites by Deer, W.A., Howie, R.A., Wise W.S. and Zussman, J." Mineral. Mag. 68(5):831-32.

Crum, JV, BJ Riley, SK Sundaram, SA Arreguin, J Matyas, MJ Schweiger, BT Rieck, and JD Vienna. 2009. Alternative Waste Forms for Electro-Chemical Salt Waste. Report No. AFCI-WAST-PMO-MIDV-2009-000293, Pacific Northwest National Laboratory, Richland, WA.

Darriet, J and J Galy. 1973. Acta Crystallographica C2:237-43.

Darriet, J, G Guillaume, K-A Wilhelmi, and J Galy. 1972. "Etude des composés $\mathrm{MVTeO}_{5}(\mathrm{M}=\mathrm{Na}, \mathrm{K}$, $\mathrm{Rb}, \mathrm{Ag}$ ). Structure cristalline de $\mathrm{NaVTeO}_{5}$." Acta Chemica Scandinavica 26:59-70.

Dollase, WA and WM Thomas. 1978a. "The crystal chemistry of silica-rich, alkali-deficient nepheline." Contributions to Mineralogy and Petrology 66(3):311-18.

Dollase, WA and WM Thomas. 1978b. "The crystal chemistry of silica-rich, alkali-deficient nepheline." Contrib. Mineral. Petrol. 66(3):311-18.

Donald, IW, BL Metcalfe, SK Fong, LA Gerrard, DM Strachan, and RD Scheele. 2007. "A glassencapsulated calcium phosphate wasteform for the immobilization of actinide-, fluoride-, and chloridecontaining radioactive wastes from the pyrochemical reprocessing of plutonium metal." Journal of Nuclear Materials 361(1):78-93.

Dong, Z, TJ White, B Wei, and K Laursen. 2002. "Model apatite systems for the stabilization of toxic metals: I, calcium lead vanadate." Journal of the American Ceramic Society 85(10):2515-22.

Duverger, C, M Bouazaoui, and S Turrell. 1997. "Raman spectroscopic investigations of the effect of the doping metal on the structure of binary tellurium-oxide glasses." Journal of Non-Crystalline Solids 220(2-3):169-77.

Ebert, WL. 2005. Testing to Evaluate the Suitability of Waste Forms Developed for Electrometallurgically-Treated Spend Sodium-Bonded Nuclear Fuel for Disposal in the Yucca Mountain Repository. Report No. ANL-05/43, Argonne National Laboratory-East, Argonne, IL.

Ebert, WL and SF Wolf. 1999. Round-Robin Testing of a Reference Glass for Low-Activity Waste Forms. Report No. ANL-99/22, Argonne National Laboratory, Argonne, Illinois.

Edwards, TG, EL Gjersing, S Sen, SC Currie, and BG Aitken. 2011. "125Te NMR chemical shifts and tellurium coordination environments in crystals and glasses in the Ge-As-Sb-Te system." Journal of Non-Crystalline Solids 357(15):3036-41.

Ewing, RC, WJ Weber, and J Clinard, F.W. 1995. "Radiation effects in nuclear waste forms for highlevel radioactive waste." Progress in Nuclear Energy 29(2):63-127.

Fayon, F, I Farnan, C Bessada, J Coutures, D Massiot, and JP Coutures. 1997. "Empirical Correlations between 207Pb NMR Chemical Shifts and Structure in Solids." Journal of the American Chemical Society 119(29):6837-43. 
Feger, CR, GL Schimek, and JW Kolis. 1999. "Hydrothermal synthesis and characterization of $\mathrm{M}_{2} \mathrm{Te}_{3} \mathrm{O}_{8}$ $(\mathrm{M}=\mathrm{Mn}, \mathrm{Co}, \mathrm{Ni}, \mathrm{Cu}, \mathrm{Zn})$ : a series of compounds with the spiroffite structure." Journal of Solid State Chemistry 143(2):246-53.

Fleet, ME. 1989. "Structures of sodium alumino-germanate sodalites $\left[\mathrm{Na}_{8}\left(\mathrm{Al}_{6} \mathrm{Ge}_{6} \mathrm{O}_{24}\right) \mathrm{A}_{2}, \mathrm{~A}=\mathrm{Cl}, \mathrm{Br}, \mathrm{I}\right]$." Acta Crystallographica C45(6):843-47.

Folger, F. 1975a. "Die Kristallstruktur von $\mathrm{Li}_{2} \mathrm{TeO}_{3}$." Journal of Inorganic and General Chemistry 411(2):103-10.

Folger, F. 1975b. "Die Kristallstruktur von $\mathrm{BaTeO}_{3}$." Journal of Inorganic and General Chemistry 411(2):111-17.

Foreman, N and DR Peacor. 1970. "Refinement of the nepheline structure at several temperatures." Journal of Solid State - New Crystal Structures 132(1-6):45-70.

Frank, S, T Barber, and M Lambregts. 2005. " Powder diffraction of sodalite in a multiphase ceramic used to immobilize radioactive waste." Powder Diffraction 20(3):212-14.

Frit, B, B Holmberg, and J Galy. 1970. "Structure cristalline de l'oxychlorure de baryum, $\mathrm{Ba}_{4} \mathrm{OCl}_{6} . "$ Acta Crystallographica B26(1):16-19.

Hanke, K. 1967. "Zinktellurit: kristallstruktur und beziehungen zu einigen seleniten." Naturwissenschaften 54(8):199.

Hanke, K. 1966. "Die kristallstruktur von $\mathrm{Zn}_{2} \mathrm{Te}_{3} \mathrm{O}_{8} . "$ Naturwissenschaften 53(11):273.

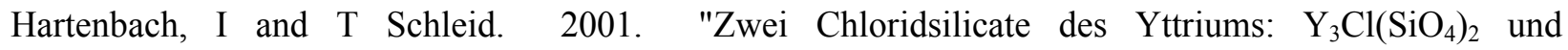
$\mathrm{Y}_{6} \mathrm{Cl}_{10}\left(\mathrm{Si}_{4} \mathrm{O}_{12}\right)$." Journal of Inorganic and General Chemistry 627(11):2493-98.

Haynes, WM, ed. Y-R Luo. 2011. CRC Handbook of Chemistry and Physics. $91^{\text {st }}$ Ed., CRC Press/Taylor and Francis, Boca Raton, FL.

Heo, J, D Lam, GH Sigel, EA Mendoza, and DA Hensley. 1992. "Spectroscopic analysis of the structure and properties of alkali tellurite glasses." Journal of the American Ceramic Society 75(2):277-81.

Hesse, K-F. 1977. "Refinement of the crystal structure of lithium polysilicate." Acta Crystallographica B33(3):901-02.

Himei, Y, A Osaka, T Nanba, and Y Miura. 1994. "Coordination change of Te atoms in binary tellurite glasses." Journal of Non-Crystalline Solids 177:164-69.

Hocdé, S, SB Jiang, X Peng, N Peyghambarian, T Luo, and M Morrell. 2004. " $\mathrm{Er}^{3+}$ doped boro-tellurite glasses for $1.5 \mu \mathrm{m}$ broadband amplification." Optical Materials 25(2):149-56.

Holland, D, J Bailey, G Ward, B Turner, P Tierney, and R Dupree. 2005. "A 125Te and 23Na NMR investigation of the structure and crystallisation of sodium tellurite glasses." Solid State Nucl. Magn. Reson. 27:16-27. 
Hruby, A. 1972. "Evaluation of glass-forming tendency by means of DTA." Czechoslovak Journal of Physics, Section B 22(11):1187-93.

Jacobson, AJ, BC Tofield, and BEF Fender. 1972. "The structures of $\mathrm{BaCeO}_{3}, \mathrm{BaPrO}_{3}$ and $\mathrm{BaTbO}_{3}$ by neutron diffraction: lattice parameter relations and ionic radii in $O$-perovskites." Acta Crystallographica B28(3):956-61.

Kabalc1, İ, G Özen, and ML Öveçoğlu. 2009. "Microstructure and crystallization properties of $\mathrm{TeO}_{2}$ $\mathrm{PbF}_{2}$ glasses." Journal of Raman Spectroscopy 40(3):272-76.

Kendrick, E and S Dann. 2004. "Synthesis, properties and structure of ion exchanged hydrosodalite." Journal of Solid State Chemistry 177(4-5):1513-19.

Kim, Y-I, M-J Jung, and KH Kim. 2000. "Application of inverse pole figure to Rietveld refinement: III. Rietveld refinement of $\mathrm{SnO}_{2}$ thin film using X-ray diffraction data." Korean Journal of Ceramics 6(4):354-58.

Knop, O and G Demazeau. 1981. "The 1-6 weberite $\mathrm{Na}_{2} \mathrm{Te}_{2} \mathrm{O}_{7}$ and some observations on compounds with the weberite structure." Journal of Solid State Chemistry 39(1):94-99.

Konan, K, C Peyratout, A Smith, J Bonnet, S Rossignol, and S Oyetola. 2009. "Comparison of surface properties between kaolin and metakaolin in concentrated lime solutions." Journal of Colloid and Interface Science 339(1):103-09.

Kondratyuk, IP, LA Muradyan, YV Pisarevskii, and VI Simonov. 1987. "Precision X-ray structure investigation of acoustooptical single crystals of $\alpha-\mathrm{TeO}_{2} . "$ Kristallografiya 32(3):354-59.

Koyama, T. 1994. "Method to synthesize dense crystallized sodalite pellet for immobilizing halide salt radioactive waste." United States Patent No. 5340506.

Kozhukharov, VS, MR Marinov, and JN Pavlova. 1978. "Phase equilibria and immiscibility in the $\mathrm{TeO}_{2}$ $\mathrm{P}_{2} \mathrm{O}_{5}$ system." Journal of Materials Science 13(5):997-1005.

Kulcu, N. 1987. "Some new metastable compounds with a defect-type calcium fluoride $\left(\mathrm{CaF}_{2}\right)$ structure." Doga: Turk Kimya Dergisi 11(1):27-31.

Larsen, FH, HJ Jakobsen, PD Ellis, and NC Nielsen. 1997. "Sensitivity-enhanced quadrupolar-echo NMR of half-integer quadrupolar nuclei. Magnitudes and relative orientation of chemical shielding and quadrupolar coupling tensors." Journal Of Physical Chemistry A 101(46):8597-606.

Larsen, FH, AS Lipton, HJ Jakobsen, NC Nielsen, and PD Ellis. 1999. "67Zn QCPMG Solid-State NMR Studies of Zinc Complexes as Models for Metalloproteins." Journal of the American Chemical Society 121(15):3783-84.

Leciejewicz, J. 1961. "The crystal structure of tellurium dioxide. A redetermination by neutron diffraction." Journal of Solid State - New Crystal Structures 116(3-6):345-53.

Levenberg, K. 1944. "A method for the solution of certain problems in least squares." Quart. Appl. Math 2:164-68. 
Lezal, D, J Bludska, J Horak, A Sklenar, S Karamazov, and M Vlcek. 2002. "Raman spectra of $\mathrm{TeO}_{2}$ $\mathrm{PbCl}_{2}$ glasses." Physics and Chemistry of Glasses 43(6):296-99.

Li, H, J Addai-Mensah, JC Thomas, and AR Gerson. 2005. "The influence of Al(III) supersaturation and $\mathrm{NaOH}$ concentration on the rate of crystallization of $\mathrm{Al}(\mathrm{OH})_{3}$ precursor particles from sodium aluminate solutions." Journal of Colloid and Interface Science 286(2):511-19.

Li, H, P Hrma, JD Vienna, M Qian, Y Su, and DE Smith. 2003. "Effects of $\mathrm{Al}_{2} \mathrm{O}_{3}, \mathrm{~B}_{2} \mathrm{O}_{3}, \mathrm{Na}_{2} \mathrm{O}$, and $\mathrm{SiO}_{2}$ on nepheline formation in borosilicate glasses: chemical and physical correlations." Journal of NonCrystalline Solids 331(1-3):202-16.

Lindner, G-G, W Massa, and D Reinen. 1995. "Structure and properties of hydrothermally synthesized thiosulfate cancrinite." Journal of Solid State Chemistry 117(2):386-91.

Lipton, AS, JA Sears, and PD Ellis. 2001. "A general strategy for the NMR observation of half-integer quadrupolar nuclei in dilute environments." Journal of Magnetic Resonance 151(1):48-59.

Lipton, AS, TA Wright, MK Bowman, DL Reger, and PD Ellis. 2002. "Solid-state Zn-67 NMR spectroscopy in bioinorganic chemistry. Spectra of four- and six-coordinate zinc pyrazolylborate complexes obtained by management of proton relaxation rates with a paramagnetic dopant." Journal of the American Chemical Society 124(20):5850-60.

Liu, Q, A Navrotsky, CF Jove-Colon, and F Bonhomme. 2007. "Energetics of cancrinite: effect of salt inclusion." Microporous and Mesoporous Materials 98(1-3):227-33.

Loopstra, BO and K Goubitz. 1986. "The structures of four caesium tellurates." Acta Crystallographica C42(5):520-23.

Mazzuca, M, J Portier, B Tanguy, F Romain, A Fadli, and S Turrell. 1995. "Raman scattering in tellurium-metal oxyde glasses." Journal of Molecular Structure 349:413-16.

McCarthy, GJ and JM Welton. 1989. "X-Ray diffraction data for $\mathrm{SnO}_{2}$. An illustration of the new powder data evaluation methods." Powder Diffraction 4(3):156-59.

McMullan, RK, S Ghose, N Haga, and V Schomaker. 1996. "Sodalite, $\mathrm{Na}_{4} \mathrm{Si}_{3} \mathrm{Al}_{3} \mathrm{O}_{12} \mathrm{Cl}$ : structure and ionic mobility at high temperatures by neutron diffraction." Acta Crystallgraphica B 52:616-27.

Meier, WM. 1973. "Symmetry Aspects of Zeolite Frameworks." In Molecular Sieves: Pt. 1 (Advances in Chemistry Series), eds. WM Meier and JB Uytterhoeven, Vol 121, pp. 39-51. Schweizerische Chemische Gesellschaft, Bern, Switzerland.

Meunier, G and J Galy. 1971. "Sur une déformation inédite du reseau de type fluorine. Structure cristalline des phases $M \mathrm{Te}_{3} \mathrm{O}_{8}(M=\mathrm{Ti}, \mathrm{Sn}, \mathrm{Hf}, \mathrm{Zr})$." Acta Crystallographica B27:602-08.

Meyer, G, J Soose, A Moritz, V Vitt, and TH Holljes. 1985. "Ternaere Halogenide der seltenen Erden vom Typ $\mathrm{A}_{2} \mathrm{MX}_{5} .\left(\mathrm{A}=\mathrm{K}, \mathrm{In}, \mathrm{NH}_{4}, \mathrm{Rb}, \mathrm{Cs} ; \mathrm{X}=\mathrm{Cl}, \mathrm{Br}, \mathrm{I}\right)$." Journal of Inorganic and General Chemistry 521:161-72.

Mineralogy Database. 2011. Accessed 2009-2011 at http://www.webmineral.com/ (last updated 10/01/2011). 
Misra, C. 1970. "Methods, apparatus: new product research, process development and design: Solubility of aluminium trihydroxide (hydrargillite) in sodium hydroxide solutions." In Chemistry and Industry, ed. TF West, Vol 19, pp. 619-23. Society of Chemical Industry, London.

Moller, CK. 1957. "A phase transition in caesium plumbochloride." Nature 180(4593):981-82.

Montagne, L, S Donze, G Palavit, JC Boivin, F Fayon, D Massiot, J Grimblot, and L Gengembre. 2001. "207Pb and 113Cd NMR and XPS characterization of PbO-PbCl2-CdCl2 glasses." Journal of NonCrystalline Solids 293-295(0):74-80.

Murugan, GS, T Suzuki, and Y Ohishi. 2006. "Raman characteristics and nonlinear optical properties of tellurite and phosphotellurite glasses containing heavy metal oxides with ultrabroad Raman bands." Journal of Applied Physics 100(2):023107-1 - 07-6.

Nachimuthu, P and R Jagannathan. 1995. "Tb3+ fluorescence as a probe of cluster formation in lead oxyfluoride glasses." Journal of Non-Crystalline Solids 183(1-2):208-11.

Neov, S, I Gerasimova, V Kozhukharov, P Mikula, and P Lukas. 1995. "Structural study of complex tellurite glasses." Journal of Non-Crystalline Solids 192-193:53-56.

Neov, S, V Kozhukharov, I Gerasimova, and B Sidzhimov. 1990. "Neutron diffraction investigation of the short range order in TeO2---BiCl3 glassy system." Journal of Non-Crystalline Solids 126(3):255-58.

Ocanto, F, R Alvarez, CU de Navarro, A Lieb, and CF Linares. 2008. "Influence of the alkalinity and $\mathrm{NO}_{3}{ }^{-} / \mathrm{Cl}^{-}$anionic composition on the synthesis of the cancrinite-sodalite system." Microporous and Mesoporous Materials 116(1-3):318-22.

Ocanto, F, E Figueredo, M Brikgi, CUd Navarro, and CF Linares. 2009. "Oxidation-reduction reactions: a novel method in the synthesis of nitrate cancrinite-type zeolites." Materials Letters 63(1):11-14.

Ott, H. 1923. "Die raumgitter der lithiumhalogenide." Physikalische Zeitschrift 24:209-13.

Park, H-S, I-T Kim, H-Y Kim, K-S Lee, S-K Ryu, and J-H Kim. 2002. "Application of Apatite Waste Form for the Treatment of Water-Soluble Wastes Containing Radioactive Elements. Part I: Investigation on the Possibility." Journal of Industrial and Engineering Chemistry 8(4):318-27.

Porter, Y and PS Halasyamani. 2003. "Syntheses, Structures, and Characterization of New Lead(II)-Tellurium(IV)-Oxide Halides: $\mathrm{Pb}_{3} \mathrm{Te}_{2} \mathrm{O}_{6} \mathrm{X}_{2}$ and $\mathrm{Pb}_{3} \mathrm{TeO}_{4} \mathrm{X}_{2}(\mathrm{X}=\mathrm{Cl}$ or $\mathrm{Br})$." Inorganic Chemistry 42(1):205-09.

Rada, S, A Dehelean, and E Culea. 2011. "FTIR and UV-VIS spectroscopy investigations on the structure of the europium-lead-tellurate glasses." Journal of Non-Crystalline Solids 357(16-17):3070-73.

Riley, BJ, JV Crum, J Matyas, JS McCloy, and WC Lepry. 2012a. "Solution-derived, chloridecontaining minerals as a waste form for alkali chlorides." Journal of the American Ceramics Society (in press).

Riley, BJ, JS McCloy, JV Crum, CP Rodriguez, CF Windisch Jr., WC Lepry, J Matyas, MP Westman, BT Rieck, JB Lang, and DA Pierce. 2011. Alternative Electrochemical Salt Waste Forms, Summary of 
FY/CY2011 Results. Report No. FCRD-SWF-2012-000028, Pacific Northwest National Laboratory, Richland, WA.

Riley, BJ, BT Rieck, JV Crum, J Matyas, J McCloy, SK Sundaram, and JD Vienna. 2010. Alternative Electrochemical Salt Waste Forms, Summary of FY2010 Results. Report No. FCRD-WAST-2010000129, Pacific Northwest National Laboratory, Richland, WA.

Riley, BJ, BT Rieck, JS McCloy, JV Crum, SK Sundaram, and JD Vienna. 2012b. "Tellurite glass as a waste form for mixed alkali-chloride waste streams: candidate materials selection and initial testing." Journal of Nuclear Materials 424(1-3):29-37.

Rodriguez, CP, J McCloy, MJ Schweiger, JV Crum, and A Winschell. 2011. Optical basicity and nepheline crystallization in high alumina glasses. Report Pacific Northwest National Laboratory, Richland, WA.

Rong, QJ, A Osaka, T Nanba, J Takada, and Y Miura. 1992. "Infrared and Raman spectra of binary tellurite glasses containing boron and indium oxides." Journal of Materials Science 27(14):3793-98.

Sakida, S, S Hayakawa, and T Yoko. 1999a. "Part 1. ${ }^{125}$ Te NMR study of tellurite crystals." Journal of Non-Crystalline Solids 243(1):1-12.

Sakida, S, S Hayakawa, and T Yoko. 2001. ${ }^{125} \mathrm{Te},{ }^{27} \mathrm{Al}$, and ${ }^{71} \mathrm{Ga} \mathrm{NMR}$ Study of $\mathrm{M}_{2} \mathrm{O}_{3}-\mathrm{TeO}_{2}(\mathrm{M}=\mathrm{Al}$ and Ga) Glasses." Journal of the American Ceramic Society 84(4):836-42.

Sakida, S, S Hayakawa, and T Yoko. 1999b. " ${ }^{125} \mathrm{Te}$ NMR Study of MO-TeO $2(\mathrm{M}=\mathrm{Mg}, \mathrm{Zn}, \mathrm{Sr}, \mathrm{Ba}$ and $\mathrm{Pb}$ ) Glasses." Journal of the Ceramic Society of Japan 107(5):395-402.

Sakida, S, S Hayakawa, and T Yoko. 1999c. "Part 2. ${ }^{125} \mathrm{Te}$ NMR study of $\mathrm{M}_{2} \mathrm{O}-\mathrm{TeO}_{2}(\mathrm{M}=\mathrm{Li}, \mathrm{Na}, \mathrm{K}, \mathrm{Rb}$ and Cs) glasses." Journal of Non-Crystalline Solids 243(1):13-25.

Sciau, P, J Lapasset, and J Moret. 1986. "Structure de la phase quadratique de $\mathrm{PbTeO}_{3} . "$ Acta Crystallographica C42(12):1688-90.

Seemann, H. 1956. "Die kristallstruktur des lithiummetasilikates, $\left(\mathrm{Li}_{2} \mathrm{SiO}_{3}\right)_{\mathrm{x}} . "$ Acta Crystallographica 9(3):251-52.

Sekiya, T, N Mochida, and S Ogawa. 1994a. "Structural study of $\mathrm{WO}_{3}-\mathrm{TeO}_{2}$ glasses." Journal of NonCrystalline Solids 176(2-3):105-15.

Sekiya, T, N Mochida, and A Ohtsuka. 1994b. "Raman spectra of $\mathrm{MO}-\mathrm{TeO}_{2}(\mathrm{M}=\mathrm{Mg}, \mathrm{Sr}, \mathrm{Ba}$ and $\mathrm{Zn})$ glasses." Journal of Non-Crystalline Solids 168(1-2):106-14.

Sekiya, T, N Mochida, A Ohtsuka, and M Tonokawa. 1992. "Raman spectra of $\mathrm{MO}_{1 / 2} \mathrm{TeO}_{2}(\mathrm{M}=\mathrm{Li}, \mathrm{Na}$, $\mathrm{K}, \mathrm{Rb}, \mathrm{Cs}$ and Tl) glasses." Journal of Non-Crystalline Solids 144:128-44.

Sekiya, T, N Mochida, and A Soejima. 1995. "Raman spectra of binary tellurite glasses containing tri- or tetra-valent cations." Journal of Non-Crystalline Solids 191(1-2):115-23.

Shannon, RD. 1976. "Revised effective ionic radii and systematic studies of interatomic distances in halides and chalcogenides." Acta Crystallographica Section A 32:751-67. 
Silva, MAP, Y Messaddeq, SJL Ribeiro, M Poulain, F Villain, and V Briois. 2001. "Structural studies on $\mathrm{TeO}_{2}-\mathrm{PbO}$ glasses." Journal of Physics and Chemistry of Solids 62(6):1055-60.

Sokolov, VO, VG Plotnichenko, and VV Koltashev. 2009. "Structure of barium chloride-oxide tellurite glasses." Journal of Non-Crystalline Solids 355(31-33):1574-84.

Sokolowski, J and A Kotarba. 1998. The structure of potassium aluminium oxide $\mathrm{KAlO}_{2}$. In Proceedings of $6^{\text {th }}$ European Powder Diffraction Conference. 954-59 pp.

Sokolowski, J and A Kotarba. 2000. "The structure of potassium aluminum oxide." Materials Science Forum 321-324:954-59.

Srinivasa, RB and SP Sanyal. 1990. "Structural and elastic properties of sodium halides at high pressure." Physical Review B: Condensed Matter and Materials Physics 42(3):1810-16.

Stratton, SW. 1924. Standard density and volumetric tables, $6^{\text {th }}$ ed., United States National Bureau of Standards, Government Printing Office, Washington.

Tait, KT, E Sokolova, FC Hawthorne, and AP Khomyakov. 2003a. "The crystal structure of nepheline." The Canadian Mineralogist 41(1):61-70.

Tait, KT, E Sokolova, FC Hawthorne, and AP Khomyakov. 2003b. "The crystal chemistry of nepheline." Canadian Mineralogist 41:61-70.

Tamazyan, RA, YA Malinovskii, and AM Il'inets. 1988. "Atomic structure and microtwinning of sodalite." Soviet Physics, Crystallography 33(3):325-29.

Tanaka, K, T Yoko, H Yamada, and K Kamiya. 1988a. "Structure and ionic conductivity of $\mathrm{LiCl}^{-\mathrm{Li}_{2} \mathrm{O}-}$ $\mathrm{TeO}_{2}$ glasses." Journal of Non-Crystalline Solids 103(2-3):250-56.

Tanaka, K, T Yoko, H Yamada, and K Kamiya. 1988b. "Structure and ionic conductivity of $\mathrm{LiCl}^{-\mathrm{Li}_{2} \mathrm{O}-}$ $\mathrm{TeO}_{2}$ glasses." Journal of Non-Crystalline Solids 103:250-56.

Tatar, D, G Özen, FB Erim, and ML Öveçoğlu. 2010. "Raman characterizations and structural properties of the binary $\mathrm{TeO}_{2}-\mathrm{WO}_{3}, \mathrm{TeO}_{2}-\mathrm{CdF}_{2}$ and ternary $\mathrm{TeO}_{2}-\mathrm{CdF}_{2}-\mathrm{WO}_{3}$ glasses." Journal of Raman Spectroscopy 41(7):797-807.

Thompson, JG, RL Withers, A Melnitchenko, and SR Palethorpe. 1998a. "Cristobalite-related phases in the $\mathrm{NaAlO}_{2}-\mathrm{NaAlSiO}_{4}$ system. I. Two tetragonal and two orthorhombic structures." Acta Crystallographica B54(5):531-46.

Thompson, JG, RL Withers, A Melnitchenko, and SR Palethorpe. 1998b. "Cristobalite-related phases in the $\mathrm{NaAlO}_{2}-\mathrm{NaAlSiO}_{4}$ system. I. Two tetragonal and two orthorhombic structures." Acta Crystallogr., Sect. B: Struct. Sci. B54(5):531-46.

Tromel, M and H Ziethen-Reichnach. 1970. "Magnesiumtellurite." Journal of Inorganic and General Chemistry 378(3):238-44. 
Vance, ER, J Davis, K Olufson, I Chironi, I Karatchevtseva, and I Farnan. 2012. "Candidate waste forms for immobilisation of waste chloride salt from pyroprocessing of spent nuclear fuel." Journal of Nuclear Materials 420(1-3):396-404.

Vienna, J. 2010. "Nuclear waste vitrification in the United States: recent developments and future options." Inernational Journal of Applied Glass Science 1(3):309-231.

Vienna, JD, J Luey, PR Hrma, DE Smith, RD Scheele, HL Wigent, and JV Crum. 1998. Glass frit development for encapsulation of rocky flats ash. Presented at Environmental Issues and Waste Management Technologies in the Ceramic and Nuclear Industries IV, Vol. 93, 399-407 pp., The American Ceramics Society, Westerville, $\mathrm{OH}$.

Vogel, W, H Burger, G Zerge, B Muller, K Forkel, G Winterstein, A Boxberger, and H Romhild. 1974. "Halogenid- und sulfathaltige telluritglaser." Silikattechnik 25(6):207-08.

Wang, D-N, Y-Q Guo, K-M Liang, and K Tao. 1999. "Crystal structure of zirconia by Rietveld refinement." Science in China - Series A: Mathematics, Physics, Astronomy \& Technological Sciences 42(1):80-86.

Wang, JS, EM Vogel, and E Snitzer. 1994. "Tellurite glass: a new candidate for fiber devices." Optical Materials 3(3): 187-203.

Weber, WJ, RP Turcotte, LR Bunnell, FP Roberts, and JH Westsik. 1979. "Radiation effects in vitreous and devitrified smulated waste glass." In Ceramics in Nuclear Waste Management, eds. TD Chikalla and JE Mendel, pp. 294-99. National Technical Information Service, Springfield, VA.

Withers, RL and JG Thompson. 1993. "Modulation wave approach to the structural parameterization and Rietveld refinement of low carnegieite." Acta Crystallographica B49(4):614-26.

Yakhkind, AK and SA Chebotarev. 1980. "Glass formation, crystallization ability, density and thermal expansion of glasses in ternary tellurite halogen systems." Fizika i Khimiya Stekla 6(2):164-69.

Yoo, TS. Personal communication. 2009. "Personal Communication by electronic mail."

Zachariasen, WH. 1929. "The crystal structure of sodium chlorate." Journal of Solid State - New Crystal Structures 71(5-6):517-29.

Zaslavsky, AI, D Kondrashev, and S Tolkachev. 1950. "The new modification of lead dioxide and the texture of anode sediments." Doklady Akademii Nauk SSSR 75:559-61. 


\section{Appendix A: Structural Notations in Tellurite Glass}




\section{Overview of Structural Units in Tellurite Glass}

A tellurite glass network consists of several different structural arrangements depending on the specific glass network modifiers, intermediates, and other additives present in the glass. X-ray diffraction (XRD) (Brady, 1956, 1957; Dimitriev et al., 1987), neutron diffraction (Neov et al., 1979), Fourier transform infrared (FTIR) spectroscopy (Dimitriev et al., 1987; Heo et al., 1992), Raman spectroscopy (Khatir et al., 1993; Duverger et al., 1997; Dimitriev et al., 1983; Kashchieva, 1984; Sekiya et al., 1994a; Chowdari and Kumari, 1999; Murugan et al., 2005, 2006; Sekiya et al., 1994b), nuclear magnetic resonance $\left({ }^{125} \mathrm{Te}\right.$ NMR) (Sakida et al., 1999c; Sakida et al., 1999a, b; Sakida et al., 2001), extended X-ray absorption fine structure (Shimizugawa et al., 1995; Yamamoto et al., 1994), and Mössbauer spectroscopy (Nishida et al., 1990) have all been used to determine the various structural configurations present in tellurite glasses. Also, observations of tellurite structural units have been discussed in the literature as they are affected by alkali (Rao and Bhat, 2001a; Bhat et al., 2004; Arnaudov et al., 1982; Akagi et al., 1999), alkaline earth (Arnaudov et al., 1982), and transition metal (Arnaudov et al., 1982; Kuzhukharov et al., 1986; Bürger et al., 1992) oxide additives.

Thus far, at least eight different Te structural units have been identified that can be broadly categorized as either asymmetrical trigonal bipyramidal $\left(t b p\right.$ or $\left.\mathrm{TeO}_{4}\right)$ or trigonal pyramidal $\left(t p\right.$ or $\left.\mathrm{TeO}_{3}\right)$ units. These different structural units are referred to with a wide variety of notations, with the most common being the polyhedral (e.g., $\mathrm{TeO}_{4}, \mathrm{TeO}_{3}$ ) and NMR (Sakida et al., 2001) notations. In the NMR notation, $N_{4}^{0}$, for example, indicates a Te polyhedron with four bonding oxygens (subscript) and a zero net charge (superscript). When modifiers are introduced into the tellurite network, the $t b p$ structural units are no longer crystallographically constrained (Akagi et al., 1999; Neov et al., 1978). Additives stabilize the vitreous state, depolymerizing it to include fractions of $\mathrm{TeO}_{3}$ or $t p$-type units, although glasses still retain the $\alpha-\mathrm{TeO}_{2}$ (tbp-type) framework structure (Arnaudov and Dimitriev, 2001). In the $t p$ units, only three oxygens are bonded to the Te atom and one of the oxygens is double-bonded. Within all of these structures exist structural moieties where some (or all) of the oxygens are non-bridging.

In addition to the polyhedral notation (e.g., $\mathrm{TeO}_{4}, \mathrm{TeO}_{3}$ ), the $\mathrm{T}_{\text {b.o. }}^{\mathrm{c}}$ notation is quite useful, where " $\mathrm{T}$ " is the Te atom, "c" is the charge on the structural unit, and "b.o." is the number of bridging oxygens included in the structural unit. These notations with their equivalence are presented in Table A.1 with pictorial representations in Figure A.1, based on compiled data from the literature (Himei et al., 1994; Sekiya et al., 1994b; Lindqvist, 1968; Rao and Bhat, 2001b; Sakida et al., 1999c; Sakida et al., 1999a). For example, according to the literature (Lindqvist, 1968), in crystalline $\alpha-\mathrm{TeO}_{2}$, the Te has a $5 \mathrm{~s}^{2}$ lone pair of electrons in the valence shell, and the preferred structural units are $\mathrm{TeO}_{4}$, more specifically $\mathrm{T}_{4}^{0}$ (neutral charge on structure with four bridging oxygens), or in our shorthand, tbp 1 (Bhat et al., 2004). In the $t p b 1$ configuration, the distance between the Te atom and both axial oxygens (i.e., Te- $\mathrm{O}_{\mathrm{ax}}$ ) is $2.08 \pm 0.02 \AA$, and the distance between the Te atom and both equatorial oxygens (i.e., $\mathrm{Te}-\mathrm{O}_{\mathrm{eq}}$ ) is $1.90 \pm 0.02 \AA$ (Lindqvist, 1968; Kuzhukharov et al., 1986). The tetragonal $\alpha-\mathrm{TeO}_{2}$ ("paratellurite") crystal consists of corner-shared trigonal bipyramidal $\mathrm{TeO}_{4}$ units and has a density of $\sim 5.75 \mathrm{~g} / \mathrm{cm}^{3}$. The $\beta-\mathrm{TeO}_{2}$ ("tellurite") crystal has an orthorhombic, layered structure, consisting of edge-shared trigonal bipyramidal $\mathrm{TeO}_{4}$ units with a higher density of $\sim 6.04 \mathrm{~g} / \mathrm{cm}^{3}$ (Patnaik, 2003). In all of the tellurite structural units, there exists a lone pair of electrons at the unoccupied equatorial position on the Te atom, a position that is generally accepted as being present in one of the $s p^{3} d$ hybridized orbitals (Lindqvist, 1968; Rao and Bhat, 2001a).

Another structural unit, known usually as $\mathrm{TeO}_{3+1}$ (denoted here $t p 0$ ) can be found in tellurite crystals when one $\mathrm{Te}-\mathrm{O}_{\mathrm{ax}}$ is stretched so that the local coordination is three with a fourth oxygen some further distance away (Kuzhukharov et al., 1986) (Figure A.1). In some sense this is intermediate between $t b p$, as it coordinated by four oxygens, and $t p$, as it has only three nearby oxygens. From another perspective, $\mathrm{TeO}_{3+1}$ units result from a continuous section of a tellurite glass chain of mostly $\mathrm{TeO}_{4} t b p$ units with $\mathrm{TeO}_{3}$ $t p$ units on either end, forming $t p 2-t b p 3-t p 2$, or a $\left[\mathrm{Te}_{3} \mathrm{O}_{8}\right]^{4-}$ unit (Sekiya et al., 1994b). If several of these sections are put together, the end $t p$ units of each section resemble "loosely associated" $\mathrm{TeO}_{3+1}$, with the "other" oxygen being contributed by the next chain fragment. This gives a $t p 0-t b p 3-t p 0$ sequence that can 
be represented as $\left[\mathrm{Te}_{3} \mathrm{O}_{8}\right]_{\infty}^{4-}$ (Sekiya et al., 1994b). A detailed explanation for the formation of structural reconfigurations in tellurite glasses has been given elsewhere (Rao and Bhat, 2001a).

Table A.1. Tellurium oxide structural units present in glass according to the literature, represented in various notations.*

\begin{tabular}{|c|c|c|c|c|c|c|}
\hline Unit & $\begin{array}{c}\text { tbp1 } \\
\text { (Himei et } \\
\text { al., 1994) } \\
\end{array}$ & $\begin{array}{c}\text { tbp2 } \\
\text { (Himei et } \\
\text { al., 1994) } \\
\end{array}$ & $\begin{array}{c}t b p 3 \\
\text { (Sekiya et al., } \\
\text { 1994b) } \\
\end{array}$ & $\begin{array}{c}t p 1 \\
\text { (Himei et } \\
\text { al., 1994) }\end{array}$ & $\begin{array}{c}t p 2 \\
\text { (Himei et al., } \\
1994) \\
\end{array}$ & $\begin{array}{c}t p 3 \\
\text { (Sakida et } \\
\text { al., 1999a) } \\
\end{array}$ \\
\hline Polyhedron & $\mathrm{TeO}_{4}$ & $\mathrm{TeO}_{4}$ & $\mathrm{TeO}_{4}$ & $\mathrm{TeO}_{3}$ & $\mathrm{TeO}_{3}$ & $\mathrm{TeO}_{3}$ \\
\hline $\begin{array}{l}\text { Alternative } \\
\text { polyhedron notation }\end{array}$ & & $\mathrm{TeO}_{3 / 2+1}^{-}$ & & $\mathrm{TeO}_{2 / 2+1}$ & $\mathrm{TeO}_{1 / 2+2}$ & $\mathrm{TeO}_{1+1+1}^{-}$ \\
\hline $\mathrm{T}_{\text {b.o. }}^{\text {chg }}$ notation & $\mathrm{T}_{4}^{0}$ & $\mathrm{~T}_{3}^{-1}$ & $\mathrm{~T}_{2}^{-2}$ & $\mathrm{~T}_{2}^{0}$ & $\mathrm{~T}_{1}^{-1}$ & $\mathrm{~T}_{0}^{-2}$ \\
\hline $\begin{array}{l}\text { NMR notation } \\
\text { (Sakida et al., 2001) }\end{array}$ & $N_{4}^{0}$ & $N_{4}^{-}$ & $N_{4}^{2-}$ & $N_{3}^{0}$ & $N_{3}^{-}$ & $N_{3}^{2-}$ \\
\hline Type & $\left(\alpha-\mathrm{TeO}_{2}\right)$ & & & (Bridging) & (Terminal) & (Isolated) \\
\hline Bonding & $\mathrm{TeO}_{4 / 2}$ & $\mathrm{O}_{3 / 2} \mathrm{Te}-\mathrm{O}^{-}$ & $\mathrm{O}_{2 / 2} \mathrm{Te}\left(-\mathrm{O}^{-}\right)-\mathrm{O}^{-}$ & $\mathrm{O}_{2 / 2} \mathrm{Te}=\mathrm{O}$ & $\mathrm{O}_{1 / 2} \mathrm{Te}(=\mathrm{O})-\mathrm{O}^{-}$ & $\mathrm{O}=\mathrm{Te}\left(-\mathrm{O}^{-}\right)-\mathrm{O}^{-}$ \\
\hline$Q^{n}$ & $\mathrm{Q}^{4}$ & $Q^{3}$ & $Q^{2}$ & $Q^{2}$ & $Q^{1}$ & $Q^{0}$ \\
\hline $\begin{array}{l}\text { Number of NBO } \\
\text { (including double } \\
\text { bonds) }\end{array}$ & 0 & 1 & 2 & 1 & 2 & 3 \\
\hline $\begin{array}{l}\text { Number of double } \\
\text { bonds }\end{array}$ & 0 & 0 & 0 & 1 & 1 & 1 \\
\hline $\begin{array}{l}\text { Equivalent in } \\
\text { network }\end{array}$ & $\mathrm{TeO}_{2}$ & {$\left[\mathrm{Te}_{2} \mathrm{O}_{5}\right]^{2-}$} & {$\left[\mathrm{TeO}_{3}\right]^{2-}$} & $\mathrm{TeO}_{2}$ & {$\left[\mathrm{Te}_{2} \mathrm{O}_{5}\right]^{2-}$} & {$\left[\mathrm{TeO}_{3}\right]^{2-}$} \\
\hline Description & \multicolumn{4}{|c|}{--------- Trigonal bypramidal --------- } & Pyramid pairs & $\begin{array}{l}\text { Isolated } \\
\text { pyramids }\end{array}$ \\
\hline
\end{tabular}

*Note that the $\mathrm{TeO}_{3+1}$ unit cannot be clearly considered by any of these models. It is nominally 4-coordinated like the other $t b p$ units, but the $4^{\text {th }}$ oxygen is farther away so it could be considered 3 -coordinated like the $t p$ units. It is therefore an intermediate configuration which in an uncharged state can be described as $\mathrm{O}_{3 / 2} \mathrm{Te}(---\mathrm{O})$ (Sekiya et al., 1994b). Depending on the number of non-bridging oxygens this structure could be described as $N_{4}^{0}$ or $N_{3}^{0}(0 \mathrm{NBO})$, $N_{4}^{-}$or $N_{3}^{-}(1 \mathrm{NBO})$, or $N_{4}^{2-}$ or $N_{3}^{2-}(2 \mathrm{NBO})$, again depending on the convention of considering it as a $t p$ or $t b p$ unit. It is not clear whether these charged structures actually exist in real glasses. Whether considered as trigonal or tetrahedral, it represents structures which have been depolymerized by additives. According to Sakida et al. (Sakida et al., 1999c) and references therein, this unit can be said to be non-existent in tellurite glasses due to the lack of expected signature in the Raman spectrum at $900 \mathrm{~cm}^{-1}$. 

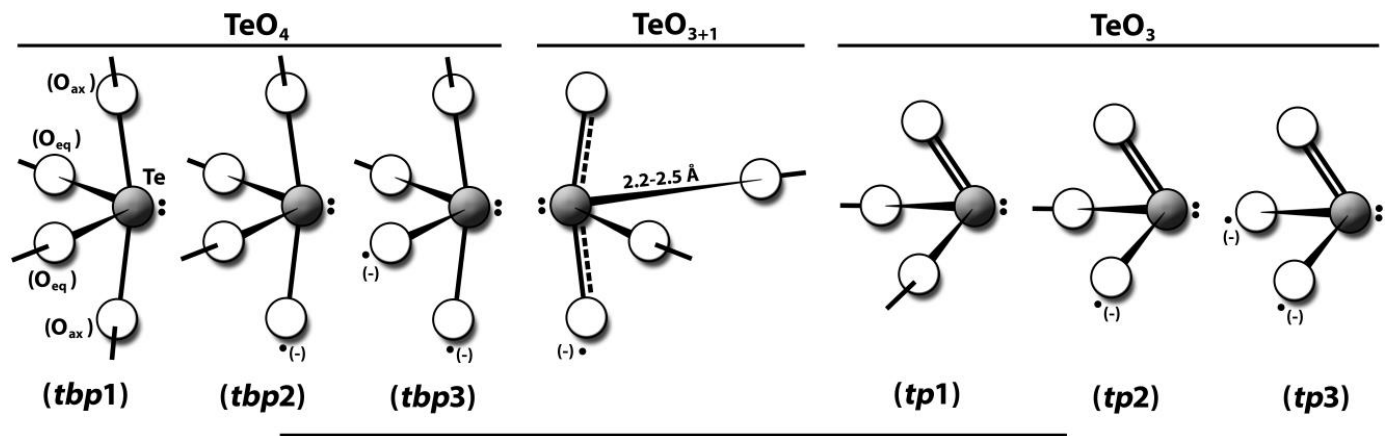

(tbp1)

(tbp2)

(tbp3)

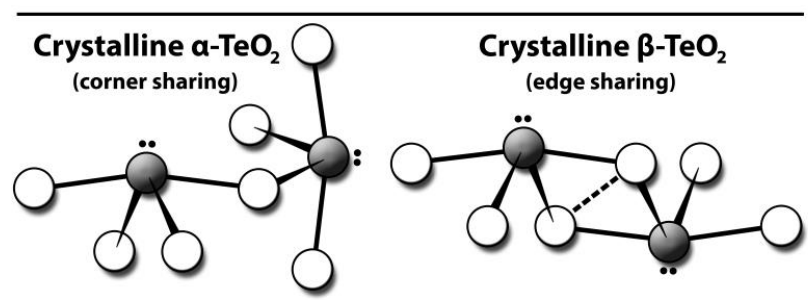

Figure A.1. $\mathrm{TeO}_{4}$ trigonal bipyramidal, $t b p$, and $\mathrm{TeO}_{3}$ trigonal pyramidal, $t p$, structural units in tellurite glasses based on representations in the literature. Species presented in order of decreasing quantity of bridging oxygens $(4 \rightarrow 0)$. Oxygen assignments are broken down into equatorial positions $\left(\mathrm{O}_{\mathrm{eq}}\right)$ and axial positions $\left(\mathrm{O}_{\mathrm{ax}}\right)$ for $t b p 1$. Similar assignments could be made for $t b p 2$ and $t b p 3$. One oxygen of $\mathrm{TeO}_{3+1}$ is unique as it is farther away. Lone pair electrons are shown as small black dots near the central dark Te atom. Non-bridging oxygen (denoted as negative sign in parenthesis) assignments were chosen arbitrarily. This illustration is not exhaustive but shows the complexity of Te-O conformations.

\section{Known effects of $\mathrm{Pb}$ and of $\mathrm{Cl}$ on tellurite glass structures}

Few structural studies of lead-tellurite glasses have been reported, however, these did provide some insight to the current study. Studies based on Raman spectroscopy of lead-tellurite glasses revealed that $\mathrm{TeO}_{3}$ structural units tend to dominate the glass structure as $\mathrm{PbO}$ is added to tellurite glass, thus $\mathrm{Pb}$ is deemed to act as a network modifier that depolymerizes the tellurite network (Khatir et al., 1993; Duverger et al., 1997; Kaur et al., 2010; Lezal et al., 2002; Komatsu and Mohri, 1999; Silva et al., 2001). However, according to $\mathrm{X}$-ray absorption spectroscopy, increasing $\mathrm{PbO}$ also promotes the conversion of 6coordinated $\mathrm{PbO}_{6}$ octahedra into 4-coordinated $\mathrm{PbO}_{4}$ tetrahedra with more non-bridging oxygens, indicating that the $\mathrm{Pb}$ also takes part in forming the glass network (Silva et al., 2001). Also, Te-O-Pb linkages exist in the network but are progressively broken by non-bridging oxygens as more $\mathrm{PbO}$ is added.

The structural effects of chlorides on the tellurite glass network have been studied to a lesser extent (Rao and Bhat, 2001a; Bhat et al., 2004; Rojo et al., 1990; Tanaka et al., 1988). In most studies where alkali chloride(s) are added to a tellurite glass, it is difficult to disentangle the effects of alkali and chloride addition on the depolymerization of the tellurite network. With XRD, Iwadate et al. (Iwadate et al., 2000) determined that the average coordination number of the Te atoms in $\mathrm{LiCl}-\mathrm{Li}_{2} \mathrm{O}$ tellurite glasses was three to four, indicating a mixture of $t p$ and $t b p$ Te species, and they suggested that $\mathrm{Cl}^{-}$substituted for $\mathrm{O}^{2-}$ in the network. Rao and Bhat (Rao and Bhat, 2001a) and Bhat et al. (Bhat et al., 2004) made several borotellurite glasses with and without $\mathrm{Li}_{2} \mathrm{O}$, including varying additions of $\mathrm{LiCl}$, and they did not expect the $\mathrm{LiCl}$ to influence the tellurite glass network structure. In their studies, adding $\mathrm{LiCl}$ resulted in a $t b p$ $\rightarrow t p$ conversion. In contrast, when studying $\mathrm{TeO}_{2}-\mathrm{Li}_{2} \mathrm{O}-\mathrm{LiCl}$ glasses through vibrational spectroscopy, Tanaka et al. (Tanaka et al., 1988) found that when $\mathrm{LiCl}$ was added to a $\mathrm{TeO}_{2}-\mathrm{Li}_{2} \mathrm{O}$ matrix, the $\mathrm{LiCl}$ formed a non-bridging oxygen bond, $\mathrm{Te}-\mathrm{Cl}_{\mathrm{eq}}$, in place of one of the $\mathrm{Te}-\mathrm{O}_{\mathrm{eq}}$ bonds ("eq" denotes an equatorial oxygen - see Figure A.1). This, in turn, strengthened the $\mathrm{Te}-\mathrm{O}_{\mathrm{ax}}$ bond and suppressed formation of the $t p$ species ("ax" denotes an axial oxygen - see Figure A.1). A thorough understanding on the effect of $\mathrm{Cl}$ in the tellurite network is still lacking. 
A very few studies have looked at systems with $\mathrm{Te}, \mathrm{Pb}, \mathrm{O}$, and $\mathrm{Cl}$. A study of the glass structure of $\mathrm{TeO}_{2^{-}}$ $\mathrm{PbCl}_{2}$ suggested that the glass was composed of tellurite $t b p$ and $t p$ units in addition to octahedral $\mathrm{PbO}_{a} \mathrm{Cl}_{b}$ and tetrahedral $\mathrm{PbO}_{4}$ units in analogy to other $\mathrm{PbO}-\mathrm{PbZ}$ glasses, where $Z$ is a halogen (Lezal et al., 2002). In mixed $\mathrm{PbO}-\mathrm{PbF}_{2}$ glass, 8-coordinated lanthanides are thought to be incorporated in the interstitials formed by six $\mathrm{PbO}_{\mathrm{x}} \mathrm{F}_{\mathrm{y}}$ octahedral (Nachimuthu and Jagannathan, 1995). Two known Te-Pb$\mathrm{O}-\mathrm{Cl}$ crystal structures are $\mathrm{Pb}_{3} \mathrm{Te}_{2} \mathrm{O}_{6} \mathrm{Cl}_{2}$ and $\mathrm{Pb}_{3} \mathrm{TeO}_{4} \mathrm{Cl}_{2}$ and the bonds are considered to be $\mathrm{Pb}-\mathrm{O}, \mathrm{Pb}-\mathrm{Cl}$, and $\mathrm{Te}-\mathrm{O}$ (Porter and Halasyamani, 2003). In other words, lead oxide-halide polyhedra (8 or 9 coordinated) are connected to tellurium oxide groups (3 or 4 coordinated). The lone pair electrons that exist both in $\mathrm{Pb}^{2+}$ and $\mathrm{Te}^{4+}$ are oriented toward the halide ions (Porter and Halasyamani, 2003).

\section{References}

Akagi, R, K Handa, N Ohtori, AC Hannon, M Tatsumisago, and N Umesaki. 1999. "High-temperature structure of $\mathrm{K}_{2} \mathrm{O}-\mathrm{TeO}_{2}$ glasses." Journal of Non-Crystalline Solids 256\&257:111-18.

Arnaudov, M and Y Dimitriev. 2001. "Study on the structural transition in binary tellurite glasses by means of reduced infrared spectra." Physics and Chemistry of Glasses 42(2):99-102.

Arnaudov, M, V Dimitrov, Y Dimitriev, and L Markova. 1982. "Infrared-spectral investigation of tellurites." Materials Research Bulletin 17(9):1121.

Bhat, MH, M Kandavel, M Ganguli, and KJ Rao. 2004. " $\mathrm{Li}^{+}$ion conductivities in boro-tellurite glasses." Bull. Mater. Sci. 27(2):189-98.

Brady, GW. 1956. "X-ray study of tellurium oxide glass." Journal of Chemical Physics 24(2):477-78.

Brady, GW. 1957. "Structure of tellurium oxide glass." Journal of Chemical Physics 27(1):300-03.

Bürger, H, K Kneipp, H Hobert, W Vogel, V Kozhukharov, and S Neov. 1992. "Glass formation, properties and structure in the $\mathrm{TeO}_{2}-\mathrm{ZnO}$ system." Journal of Non-Crystalline Solids 151:134-42.

Chowdari, BVR and PP Kumari. 1999. "Raman spectroscopic study of ternary silver tellurite glasses." Materials Research Bulletin 34(2):327-42.

Dimitriev, Y, V Dimitrov, E Gatev, E Kashchieva, and H Petkov. 1987. "Effect of the mode formation on the structure of tellurite glasses." Journal of Non-Crystalline Solids 95-96(2):937-44.

Dimitriev, Y, E Kashchieva, I Ivanova, and D Khristova. 1983. "Liquidation in three-component tellurite systems $\mathrm{TeO}_{2}-\mathrm{B}_{2} \mathrm{O}_{3}-\mathrm{M}_{\mathrm{n}} \mathrm{O}_{\mathrm{m}} \cdot \mathrm{M}_{\mathrm{n}} \mathrm{O}_{\mathrm{m}}=\mathrm{Al}_{2} \mathrm{O}_{3}, \mathrm{Ga}_{2} \mathrm{O}_{3}, \mathrm{Cr}_{2} \mathrm{O}_{3}, \mathrm{CuO}, \mathrm{Ag}_{2} \mathrm{O}, \mathrm{MoO}_{3}, \mathrm{Sb}_{2} \mathrm{O}_{3}$." Stroit. Mater. Silikat. Prom. 24(9):24.

Duverger, C, M Bouazaoui, and S Turrell. 1997. "Raman spectroscopic investigations of the effect of the doping metal on the structure of binary tellurium-oxide glasses." Journal of Non-Crystalline Solids 220(2-3):169-77.

Heo, J, D Lam, GH Sigel, EA Mendoza, and DA Hensley. 1992. "Spectroscopic analysis of the structure and properties of alkali tellurite glasses." Journal of the American Ceramic Society 75(2):277-81.

Himei, Y, A Osaka, T Nanba, and Y Miura. 1994. "Coordination change of Te atoms in binary tellurite glasses." Journal of Non-Crystalline Solids 177:164-69. 
Iwadate, Y, H Kenmotsu, T Hattori, S Nishiyama, K Fukushima, N Umesaki, T Nakazawa, and K Noda. 2000. "X-ray diffraction study of the short-range structure of $\mathrm{LiCl}-\mathrm{Li}_{2} \mathrm{O}-\mathrm{TeO}_{2}$ glasses." Journal of Alloys and Compounds 305:130-35.

Kashchieva, E. 1984. "Phase separation in tellurite systems." Sofia University, Sofia, Bulgaria: Thesis.

Kaur, A, A Khanna, C Pesquera, F González, and V Sathe. 2010. "Preparation and characterization of lead and zinc tellurite glasses." Journal of Non-Crystalline Solids 356(18-19):864-72, 10.1016/j.jnoncrysol.2010.01.005.

Khatir, S, F Romain, J Portier, S Rossignol, B Tanguy, JJ Videau, and S Turrell. 1993. "Raman studies of recrystallized glasses in the binary $\mathrm{TeO}_{2}-\mathrm{PbO}$ system." Journal of Molecular Structure 298:13-16.

Komatsu, T and H Mohri. 1999. "Raman scattering spectra and optical properties of tellurite glasses and crystalline phases containing $\mathrm{PbO}$ and CdO." Physics and Chemistry of Glasses 40:257-63.

Kuzhukharov, V, H Burger, S Neov, and B Sidzhimov. 1986. "Atomic arrangement of a zinc-tellurite glass." Polyhedron 5(3):771-77.

Lezal, D, J Bludska, J Horak, A Sklenar, S Karamazov, and M Vlcek. 2002. "Raman spectra of $\mathrm{TeO}_{2}-$ $\mathrm{PbCl}_{2}$ glasses." Physics and Chemistry of Glasses 43(6):296-99.

Lindqvist, O. 1968. "Refinement of the structure of $\alpha-\mathrm{TeO}_{2} . "$ Acta Chemica Scandinavica 22:977-82.

Murugan, GS, T Suzuki, and Y Ohishi. 2005. "Phospho-tellurite glasses containing heavy metal oxides for ultrabroad band fiber Raman amplifiers." Applied Physics Letters 86(22):221109-1-3.

Murugan, GS, T Suzuki, and Y Ohishi. 2006. "Raman characteristics and nonlinear optical properties of tellurite and phosphotellurite glasses containing heavy metal oxides with ultrabroad Raman bands." Journal of Applied Physics 100(2):023107-1 - 07-6.

Nachimuthu, P and R Jagannathan. 1995. "Tb3+ fluorescence as a probe of cluster formation in lead oxyfluoride glasses." Journal of Non-Crystalline Solids 183(1-2):208-11, 10.1016/0022-3093(94)005869.

Neov, S, I Gerassimova, K Krezhov, B Sydzhimov, and V Kozhukharov. 1978. "Atomic arrangement in tellurite glasses studied by neutron diffraction." physica status solidi (a) 47(2):743-50.

Neov, S, V Kozhukharov, I Gerasimova, K Krezhov, and B Sidzhimov. 1979. "A model for structural recombination in tellurite glasses." Journal of Physics C: Solid State Physics 12(13):2475-85.

Nishida, T, M Yamada, H Ide, and Y Takashima. 1990. "Correlation between the structure and glass transition temperature of potassium, magnesium and barium tellurite glasses." Journal of Materials Science 25(8):3546-50.

Patnaik, P. 2003. Handbook of Inorganic Chemicals, McGraw-Hill, New York.

Porter, Y and PS Halasyamani. 2003. "Syntheses, Structures, and Characterization of New Lead(II)-Tellurium(IV)-Oxide Halides: $\mathrm{Pb}_{3} \mathrm{Te}_{2} \mathrm{O}_{6} \mathrm{X}_{2}$ and $\mathrm{Pb}_{3} \mathrm{TeO}_{4} \mathrm{X}_{2}(\mathrm{X}=\mathrm{Cl}$ or $\mathrm{Br})$." Inorganic Chemistry 42(1):205-09, 10.1021/ic025750j.

Rao, KJ and MH Bhat. 2001a. "Investigation of lithium chloride-lithium borate-tellurium dioxide 
glasses: an example of complex anionic speciation." Phys. Chem. Glasses 42(3):255-64.

Rao, KJ and $\mathrm{MH}$ Bhat. 2001b. "Investigation of lithium chloride-lithium borate-tellurium dioxide glasses: an example of complex anionic speciation." Physics and Chemistry of Glasses - European Journal of Glass Science and Technology Part B 42(3):255-64.

Rojo, JM, J Sanz, JM Reau, and B Tanguy. 1990. "Influence of ion distribution on the ionic conductivity of lithium tellurite glasses $\left(\mathrm{Li}_{2} \mathrm{O}-\mathrm{TeO}_{2}\right.$ and $\left.\mathrm{LiF}-\mathrm{TeO}_{2}\right)$." Journal of Non-Crystalline Solids 116(2-3):16774.

Sakida, S, S Hayakawa, and T Yoko. 2001. ${ }^{125} \mathrm{Te},{ }^{27} \mathrm{Al}$, and ${ }^{71} \mathrm{Ga}$ NMR Study of $\mathrm{M}_{2} \mathrm{O}_{3}-\mathrm{TeO}_{2}(\mathrm{M}=\mathrm{Al}$ and Ga) Glasses." Journal of the American Ceramic Society 84(4):836-42, 10.1111/j.11512916.2001.tb00749.x.

Sakida, S, S Hayakawa, and T Yoko. 1999a. "Part 1. ${ }^{125}$ Te NMR study of tellurite crystals." Journal of Non-Crystalline Solids 243(1):1-12.

Sakida, S, S Hayakawa, and T Yoko. 1999b. " ${ }^{125}$ Te NMR Study of MO-TeO 2 (M=Mg, Zn, Sr, Ba and $\mathrm{Pb}$ ) Glasses." Journal of the Ceramic Society of Japan 107(5):395-402.

Sakida, S, S Hayakawa, and T Yoko. 1999c. "Part 2. ${ }^{125}$ Te NMR study of $\mathrm{M}_{2} \mathrm{O}-\mathrm{TeO}_{2}(\mathrm{M}=\mathrm{Li}, \mathrm{Na}, \mathrm{K}, \mathrm{Rb}$ and Cs) glasses." Journal of Non-Crystalline Solids 243(1):13-25.

Sekiya, T, N Mochida, and S Ogawa. 1994a. "Structural study of $\mathrm{WO}_{3}-\mathrm{TeO}_{2}$ glasses." Journal of NonCrystalline Solids 176(2-3):105-15.

Sekiya, T, N Mochida, and A Ohtsuka. 1994b. "Raman spectra of $\mathrm{MO}-\mathrm{TeO}_{2}(\mathrm{M}=\mathrm{Mg}, \mathrm{Sr}, \mathrm{Ba}$ and $\mathrm{Zn})$ glasses." Journal of Non-Crystalline Solids 168(1-2):106-14.

Shimizugawa, Y, T Maeseto, S Suehara, S Inoue, and A Nukui. 1995. "EXAFS and RDF studies of $\mathrm{TeO}_{2}-\mathrm{Li}_{2} \mathrm{O}$ glasses." Journal of Materials Research 10(2):405-10.

Silva, MAP, Y Messaddeq, SJL Ribeiro, M Poulain, F Villain, and V Briois. 2001. "Structural studies on $\mathrm{TeO}_{2}-\mathrm{PbO}$ glasses." Journal of Physics and Chemistry of Solids 62(6):1055-60.

Tanaka, K, T Yoko, H Yamada, and K Kamiya. 1988. "Structure and ionic conductivity of $\mathrm{LiCl}^{-\mathrm{Li}_{2} \mathrm{O}-}$ $\mathrm{TeO}_{2}$ glasses." Journal of Non-Crystalline Solids 103:250-56.

Yamamoto, H, H Nasu, J Matsuoka, and K Kamiya. 1994. "X-ray absorption fine structure (XAFS) study on the coordination of $\mathrm{Te}$ in $\mathrm{PbO}-\mathrm{TiO}_{2}-\mathrm{TeO}_{2}$ glasses with high third-order optical non-linearity " Journal of Non-Crystalline Solids 170(1):87-96. 